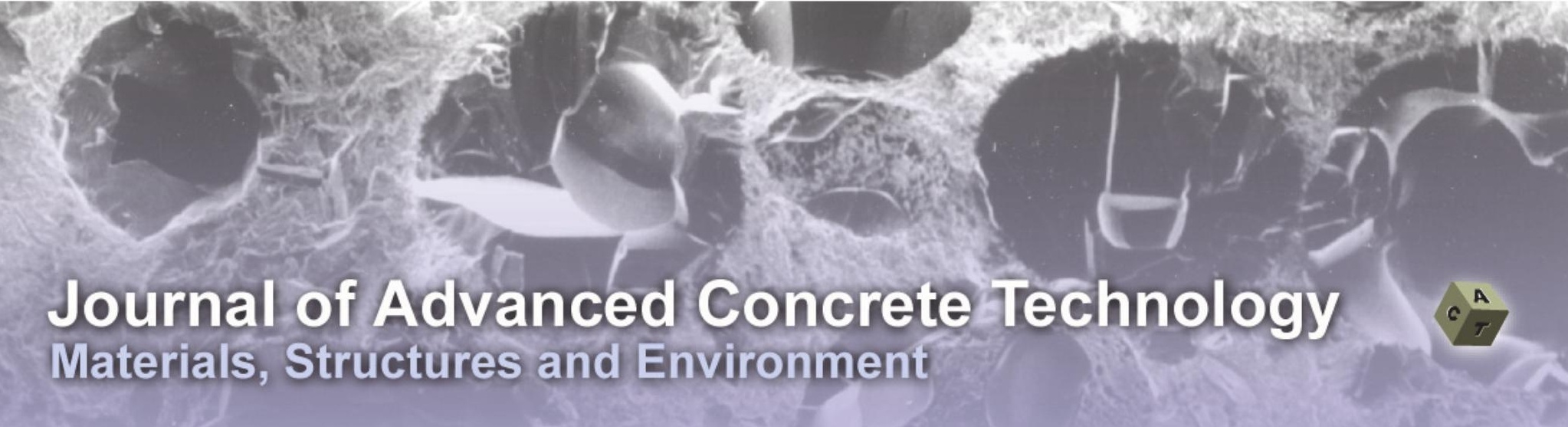

Special issue

\title{
Advances in Self-healing Cementitious Materials
}

Edited by T. Kanda

Invited Paper

Development of Engineered Self-Healing and Self-Repairing Concrete-State-of-the-Art Report

Hirozo Mihashi, Tomoya Nishiwaki

$170-184$

Self Healing of Concrete Structures - Novel Approach Using Porous Network Concrete

Senot Sangadji, Erik Schlangen

185-194

Robust Self-Healing Concrete for Sustainable Infrastructure

Victor C. Li, Emily Herbert

207-218

Scientific Paper

Enhanced Long-Term Resistance of Concrete with Marine Sessile Organisms to Chloride Ion Penetration Yuichiro Kawabata, Ema Kato, Mitsuyasu Iwanami

$151-159$

Experimental Study on Self-Healing Capability of FRCC Using Different Types of Synthetic Fibers

Tomoya Nishiwaki, Marina Koda, Makoto Yamada, Hirozo Mihashi, Takatsune Kikuta

195-206

Development of High-Durability Concrete with a Smart Artificial Lightweight Aggregate

Jinhwan Jeon, Tetsushi Kanda, Haruki Momose, Hirozo Mihashi

$231-239$

Experimental Investigation on Reaction Rate and Self-healing Ability in Fly Ash Blended Cement Mixtures Seung Hyun Na, Yukio Hama, Madoka Taniguchi, Osamu Katsura, Takahiro Sagawa, Mohamed Zakaria 240-253

Self Healing of Longitudinal Cracks in Utility Concrete Pole

Akira Hosoda, Takayuki Higuchi, Masataka Eguchi, Haruaki Yoshida, Hayato Aoki

$278-284$

Recovery of Protective Performance of Cracked Ultra High Performance Strain Hardening Cementitious Composites (UHP-SHCC) due to Autogenous Healing

Minoru Kunieda, Kang Choonghyun, Naoshi Ueda, Hikaru Nakamura

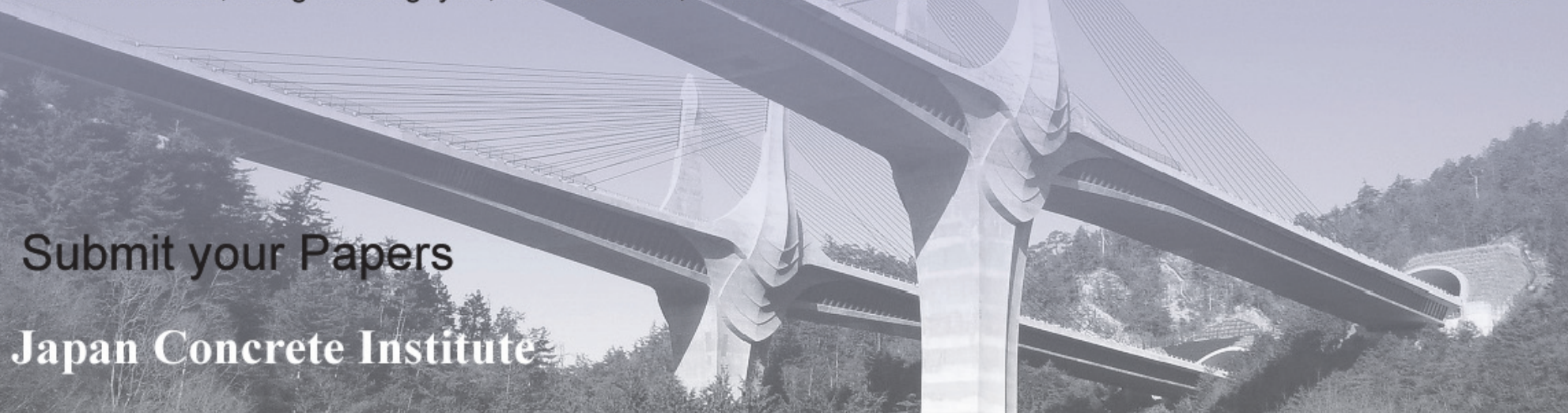




\section{Preface}

This special issue is aiming at summarizing recent progress in self-healing cementitious materials and at motivating further development in the relevant research. It took a long time since the efforts to develop self-healing materials working as intelligent materials drew strong attention worldwide in the research communities. Research and development of self-healing performance have been progressing on varieties of materials including metals, non-metals, inorganic and organic materials, and composite materials. Particularly for cement-based materials, based on the background of epoch-making ideas and existing research results, it has become possible to introduce some self-healing functions and their combined mechanisms. These self-healing cementitious materials have potentials to renovate existing structural designs and maintaining schemes and contribute to service life extension and environmental impact reduction. We expect this special issue could support to realize the potential.

This special issue was worked out under a strong support of $\mathrm{JCl}$ Technical Committee on Self-healing / Repairing Technology in Cement-based Materials (JCl TC-091) chaired by Prof. S. Igarashi, Kanazawa University. This committee's activities included 1) quantitative evaluation of self-healing performance such as water leakage control and loading damage recovery, 2) investigation into self-healing mechanism, and 3) application of non-destructive test to identify self-healing results. Committee members of the TC are listed below.

Koichi Maekawa, Editor-in-Chief of ACT

\section{TC-091 committee members}

$\begin{array}{lll}\text { Chairman } & \text { Shin-ichi IGARASHI } & \text { Kanazawa University } \\ \text { Co Chairman } & \text { Toshiharu KISHI } & \text { The University of Tokyo } \\ \text { Secretary } & \text { Minoru KUNIEDA } & \text { Nagoya University } \\ \text { Secretary } & \text { Tomoya NISHIWAKI } & \text { Tohoku University } \\ \text { Member } & \text { Tae-Ho AHN } & \text { The University of Tokyo } \\ \text { Member } & \text { Hiroshi INADA } & \text { Shimizu Corporation } \\ \text { Member } & \text { Kei-ichi IMAMOTO } & \text { Tokyo University of Science } \\ \text { Member } & \text { Yuichi OTABE } & \text { Sumitomo Osaka Cement Co., Ltd. } \\ \text { Member } & \text { Yuichiro KAWABATA } & \text { Port and Airport Research Institute } \\ \text { Member } & \text { Tetsushi KANDA } & \text { Kajima Corporation } \\ \text { Member } & \text { Kaoru KOBAYASHI } & \text { East Japan Railway Company } \\ \text { Member } & \text { Yoshinori GONDAI } & \text { Sendai National College of Technology } \\ \text { Member } & \text { Takahiro SAGAWA } & \text { Nittetsu Cement Co., Ltd. } \\ \text { Member } & \text { Yoshie SATO } & \text { Sumitomo Osaka Cement Co., Ltd. } \\ \text { Member } & \text { Madoka TANIGUCHI } & \text { Northern Regional Building Research Institute } \\ \text { Member } & \text { Sanjay PAREEK } & \text { Nihon University } \\ \text { Member } & \text { Takayuki HIGUCHI } & \text { Denki Kagaku Kogyo Kabushiki Kaisha } \\ \text { Member } & \text { Takashi HITOMI } & \text { Obayashi Corporation } \\ \text { Member } & \text { Akira HOSODA } & \text { Yokohama National University } \\ \text { Member } & \text { Ippei MARUYAMA } & \text { Nagoya University } \\ \text { Member } & \text { Haruaki YOSHIDA } & \text { Nihon Network Support Co., Ltd. } \\ \text { Member } & \text { Takeshi WATANABE } & \text { Tokushima University } \\ \text { Associate Member } & \text { Jinhwan JEON } & \text { Kajima Corporation }\end{array}$




\title{
Enhanced Long-Term Resistance of Concrete with Marine Sessile Organisms to Chloride Ion Penetration
}

\author{
Yuichiro Kawabata ${ }^{1}$, Ema Kato ${ }^{2}$ and Mitsuyasu Iwanami ${ }^{3}$
}

\begin{abstract}
Reinforced concrete (RC) structures in marine environments are generally affected by harsh marine environmental actions, resulting in early performance degradation mainly due to chloride-induced deterioration. In such conditions, corrosion of rebar progresses rapidly, and also the cross-sectional area of rebar is reduced and consequently structural performance of RC structures will be degraded. In contrast, the surface of concrete structures is often covered with many marine sessile organisms under marine tidal and submerged conditions. These marine sessile organisms have been empirically known to enhance the durability of concrete though the effectiveness is not appropriately evaluated.

This paper describes the long-term resistance of concrete with marine sessile organisms to chloride ion penetration in concrete. The effect and its sustainability of marine sessile organisms on chloride ion penetration in concrete were investigated through field exposure tests and laboratory tests. From the test results, the basal membrane, which is a matrix of marine sessile organisms, adheres to concrete strongly on a long-term basis though some gaps between concrete and the basal membrane can be observed. In addition, experimental results and simplified simulation clarified that the attachment of marine sessile organisms can enhance the long-term resistance of concrete to chloride ion penetration.
\end{abstract}

\section{Introduction}

Marine reinforced concrete structures are generally affected by harsh marine environmental actions, resulting in early performance degradation mainly due to chloride attack. In such conditions, corrosion of steel bar progresses rapidly, reducing the cross-sectional area of rebar. Consequently, the structural performance of reinforced concrete structures will be degraded.

In the process of chloride-induced corrosion of reinforced concrete, chloride ion penetration into concrete has been discussed all over the world and remains a controversial issue. In order to evaluate the service life of a structure, development of a reliable predictive model of chloride ion penetration in concrete is required. The error function solution to Fick's second law is usually used for practical prediction though many models are proposed in the research field (Nilsson 2009). Chloride ion penetration mechanisms are complex since there are so many factors involved, such as interactions between cement hydrates and chloride, moisture condition of concrete, and time-dependency of diffusion coefficient, to name a few. In addition, the penetration process of concrete immersed in real seawater is different from that immersed in a simulated sodium chloride solution. One

\footnotetext{
${ }^{1}$ Researcher, Structural Mechanics Division, Port and Airport Research Institute, Yokosuka, Japan. E-mail: kawabata-y@pari.go.jp

${ }^{2}$ Senior Researcher, Structural Mechanics Division, Port and Airport Research Institute, Yokosuka, Japan.

${ }^{3}$ Head, Structural Mechanics Division, Port and Airport Research Institute, Yokosuka, Japan.
}

example is interactions between cement hydrates and minor ions in seawater. Buenfeld and Newman (1986) pointed out that the surface skin consisting of a thin layer of brucite of around $30 \mu \mathrm{m}$ thickness and overlaying thick layer of aragonite can be formed in seawater. This surface skin causes a significant reduction in permeability of concrete. Advanced predictive models considering these phenomena are being developped.

Meanwhile, under marine tidal and submerged environment, the surface of concrete structure is often covered with an abundance of marine sessile organisms such as barnacles, oysters and mussels. It is thought that attachment of these marine sessile organisms is undesired from the aesthetic viewpoint, as is seen by what they are called: "marine fouling organisms." Marine sessile organisms also impede visual inspection of the structure, consequently generating misleading inspection results. Therefore, when carrying out inspection of existing marine structures, the marine sessile organisms is removed from the concrete surface, if necessary (Port and Airport Research Institute 2007).

Regarding the durability of concrete with marine sessile organisms, the previous papers (Iwanami et al. 2002, Maruya et al. 2003) reported the interesting experimental result that the concrete covered with marine sessile organisms, especially barnacles, had a high resistance to chloride-induced corrosion. The basal membrane of a barnacle is significantly denser than concrete, so the ingress of chloride ion and oxygen can be reduced. This result suggested that there is a possibility that the attachment of marine sessile organisms can contribute to high durability of concrete structures.

The possibility mentioned above poses the question: How long can this effect be maintained? Depending on 
the environmental conditions, the life-span of marine sessile organisms ranges from 1 to 10 years (The sessile organisms society of Japan 2006), apparently shorter than that of concrete structures. In addition, the desiccation process breaks up adhesions between barnacles and attached substances (Wiegemann and Watermann 2004). This point suggests that there is a risk that sufficient effectiveness cannot be attained under the tidal environment for long-term service.

The adhesion between marine sessile organisms and concrete is attributed to the formation of basal membrane consisting of calcite and several types of protein substances. The effect of a cyclic wetting and drying process under the tidal environment condition on adhesions between marine sessile organisms and concrete has not been clarified. Therefore, it is necessary to make clear the long-term resistance of concrete with marine sessile organisms to chloride ion penetration since it can be construed that the adhesion of basal membrane strongly affects the chloride ion penetration.

This paper describes the extensive work of the research reported by previous pioneering works (Iwanami et al. 2002, Maruya et al. 2003). The long-term exposure testing spanning about 10 years and experiments were carried out in order to determine the long-term resistance of concrete with marine sessile organisms to chloride ion penetration.

\section{Details of the experiment}

\subsection{Test specimens}

Reinforced concrete (RC) beams were casted for long-term exposure testing. The size of the test specimens was $200 \times 300 \times 2400 \mathrm{~mm}$. The mixture proportion of the concrete is summarized in Table 1. The water to cement ratio was $49.5 \%$ and ordinary portland cement was used. The compressive strength of the concrete at 37 days was $30.5 \mathrm{~N} / \mathrm{mm}^{2}$.

The test specimens were subjected to three types of exposure conditions as shown in Table 2: (Type A) 9 years and 3 months in Shimizu Port; (Type B) 9 years in the controlled seawater-splashing exposure site in the

Table 1 Mixture proportion of concrete.

\begin{tabular}{|c|c|c|c|c|c|}
\hline \multirow{2}{*}{$\begin{array}{l}\mathrm{W} / \mathrm{C} \\
(\%)\end{array}$} & \multirow{2}{*}{$\begin{array}{c}\mathrm{s} / \mathrm{a} \\
(\%)\end{array}$} & \multicolumn{4}{|c|}{ Unit content $\left(\mathrm{kg} / \mathrm{m}^{3}\right)$} \\
\cline { 3 - 6 } & $\mathrm{W}$ & $\mathrm{C}$ & $\mathrm{S}$ & $\mathrm{G}$ \\
\hline 49.5 & 41.8 & 161 & 326 & 749 & 1086 \\
\hline
\end{tabular}

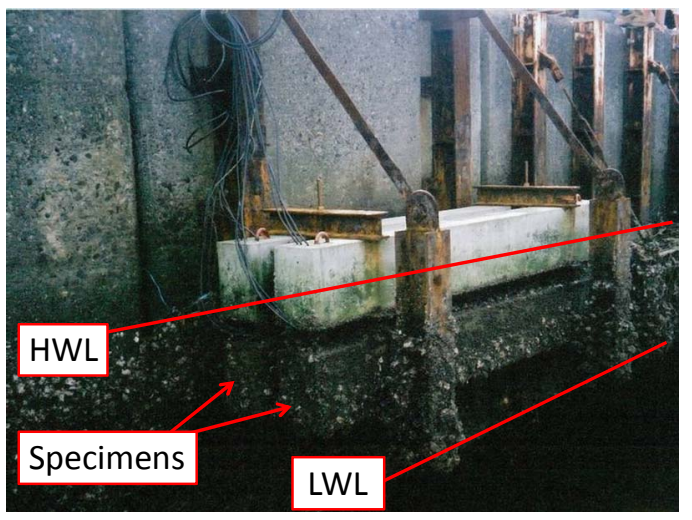

Fig. 1 The location of the test specimens in Shimizu Port.

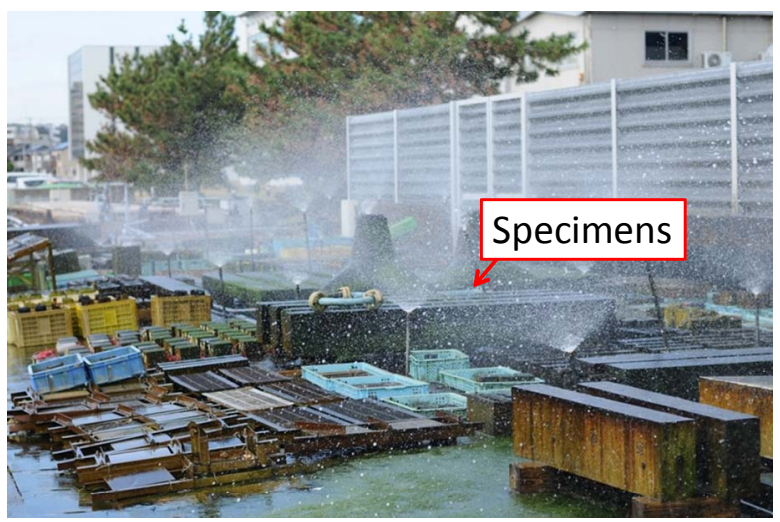

Fig. 2 Overview of PARI exposure site.

Port and Airport Research Institute (PARI exposure site) after 7 months in Shimizu Port; (Type C) 9 years and 7 months in the PARI exposure site. Shimizu Port is located in inner Suruga Bay. Tidal variation and annual mean temperature were $1.6 \mathrm{~m}$ and $16.0^{\circ} \mathrm{C}$, respectively. According to the investigation of existing $\mathrm{RC}$ structure near the exposure site, barnacles, oysters and mussels can be detected as marine sessile organisms. Figure 1 shows the location of the test specimens in Shimizu Port with the tidal range. The test specimens were immersed in seawater for approximately 15 hours. Figure 2 shows an overview of the PARI exposure site. At this site, natural seawater was pumped up from Kurihama Bay, which is the inner bay area of Tokyo Bay, and sprayed onto the specimens twice a day for 3 hours each and no marine sessile organisms were attached on the concrete surface. Therefore, the drying period per a day in the PARI exposure site was 18 hours or 2 times longer than that in

Table 2 Exposure condition.

\begin{tabular}{|c|c|c|c|}
\hline & Exposure condition & $\begin{array}{c}\text { Attachment of } \\
\text { marine sessile organisms }\end{array}$ & $\begin{array}{c}\text { Attachment area of } \\
\text { marine sessile organisms } \mathrm{A}_{\mathrm{ad}}(\%)\end{array}$ \\
\hline Type A & 9 years and 3 months in Shimizu Port & $\begin{array}{c}\text { 9 years and 3 months } \\
\text { continuously }\end{array}$ & $85-96$ \\
\hline Type B & $\begin{array}{c}\text { 9 years in PARI exposure site } \\
\text { after 7 months in Shimizu Port }\end{array}$ & First 7 months only & $42-51$ \\
\hline Type C & $\begin{array}{c}\text { 9 years and 7 months in } \\
\text { PARI exposure site }\end{array}$ & No & 0 \\
\hline
\end{tabular}


general tidal environments. The PARI exposure site enhanced the drying process of concrete so that detachment between marine sessile organisms and concrete would be accelerated.

After exposure testing, concrete cores were extracted from the RC beams. By using these core samples, the experimental programs described in the following sections were carried out.

\subsection{Attached area measurement}

The attached and detached areas of marine sessile organisms were observed and traced on tracing paper. By using image analysis, the percentage of the sum of attached and detached areas to the whole measured area, $A_{a d}$, was calculated.

\subsection{Electrical migration test}

Electrical migration testing as an accelerated test is carried out to evaluate the chloride ion penetration in concrete. Cylinder specimens with a diameter of $100 \mathrm{~mm}$ were cored from the RC beam. After being saturated with water, the test specimen is sandwiched between two chambers. The specimen which had a thickness of $30 \mathrm{~mm}$, included one surface with marine sessile organisms. As an anodic chamber solution, saturated calcium hydroxide was used, whereas a $5.0 \%$ sodium chloride solution was used as the cathodic chamber solution. The surface with marine sessile organisms was fixed to the cathodic chamber. Direct current of $15 \mathrm{~V}$ was applied between the anodic and the cathodic chambers. The sample solution was taken from the anodic chamber periodically to measure the chloride ion concentration. At a steady state, the effective chloride ion diffusion coefficient, $D_{e}$, was calculated using the Nernst-Planck's equation with an increasing rate of chloride concentration in the cathodic chamber solution (Japan Society of Civil Engineers 2005).

\subsection{Chloride ion content measurement}

Chloride ion content in concrete was measured at depths of 0-5, 5-10, 10-20, 20-30, 30-40, 40-50 and 80-90 mm from the surface. After being cut, the samples were powdered by a vibrating mill. Total chloride concentration was measured as per JCI-SC4.

\subsection{SEM-EDS analysis}

SEM observation and EDS analysis were carried out in order to investigate the interface between marine sessile organisms and concrete. Analyses were conducted with an accelerating voltage of $15 \mathrm{kV}$ and with a beam current of $300 \mathrm{pA}$.

\section{Results and discussions}

\subsection{Attachment of marine sessile organisms}

Figure 3 shows the concrete surface of Type B, in which the detachment between marine sessile organisms and concrete surface was accelerated. During exposure in

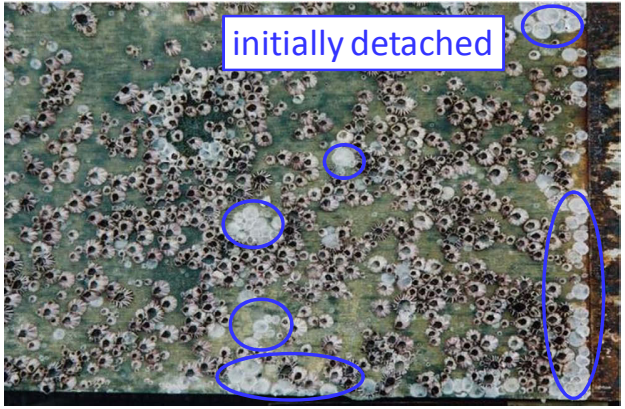

(a) 4 months in PARI exposure site

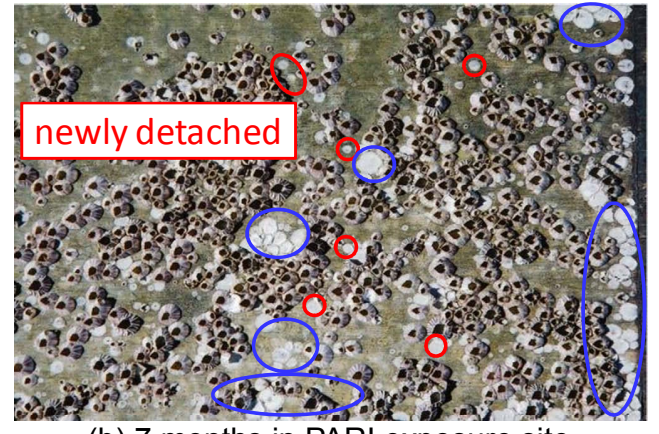

(b) 7 months in PARI exposure site

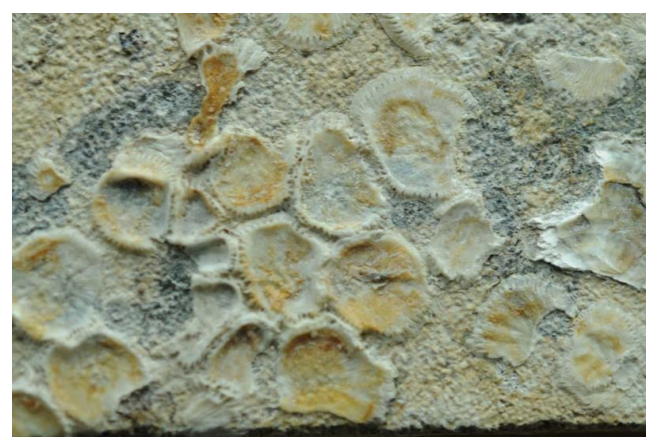

(c) Basal membrane

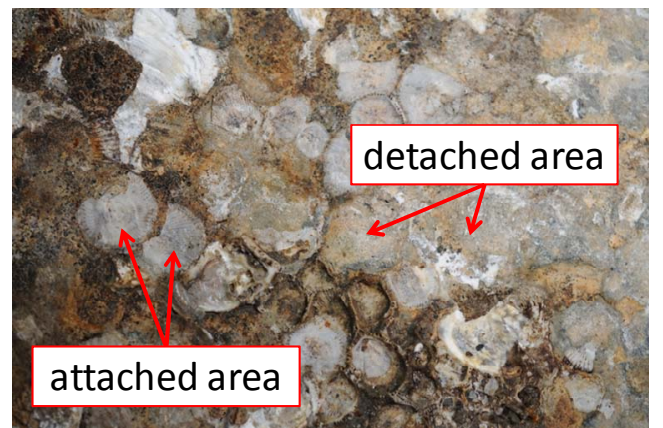

(d) Detachment of basal membrane (after exposure)

Fig. 3 Adhesion of marine sessile organisms to Type B.

Shimizu Port for 7 months, marine sessile organisms were attached on the concrete surface. The main sessile organisms were barnacles and some oysters could be found on the concrete surface. After 4 months of exposure at the PARI exposure site, detachment of barnacles could be found as shown in Fig. 3(a). This might be 
caused by transportation from Shimizu Port to the PARI exposure site. Thereafter, newly detachment derived from physical or chemical action could be found as shown in Fig. 3(b). The basal membrane, however, could adhere to the concrete surface (Fig. 3(c)). Even after the end of the life-span of marine sessile organisms, the basal membrane can show strong adhesion to attached substances (The sessile organisms society of Japan 2006). After exposure testing, more than half of basal membranes were detached (Fig. 3(d)). White gel-like deposits, however, could be detected in the detached area. On the contrary, Type A, in which marine sessile organisms can attach continuously, showed an abundance of marine sessile organisms attached on the concrete surface after exposure. The attached area of marine sessile organisms reached over $85 \%$ of concrete surface. In Type C concrete, no attachment of marine sessile organisms was observed.

In order to investigate adhesion between the basal membrane and concrete, the interface was observed by SEM and analyzed by EDS. Figure 4 shows the backscattered electron image (BEI) of Type A concrete. Figure 4(a) shows the interface of attached and non-attached areas. Basal membrane adheres to concrete, depending on asperity, voids of the concrete surface. It is also clear that the surface of non-attached area was damaged compared to the attached area. The damage is attributable to seawater attack (Gjorv, 1971). C-S-H gel converting to $\mathrm{M}-\mathrm{S}-\mathrm{H}$ gel and ettringite formation due to penetration of $\mathrm{Mg}^{2+}$ and $\mathrm{SO}_{4}{ }^{2-}$ contained in seawater induces damage to the concrete surface. Attachment of marine sessile organisms can suppress not only chloride ion penetration but also deterioration due to seawater attack. As shown in Fig. 4(b), the basal membrane can also adhere well to the mesoscopically smooth surface of concrete though some gaps between basal membrane and concrete can be detected. Figure 5 shows a magnified image of the interface between basal membrane and concrete, with distribution of elements. In the microscopic view, physical adhesion is seen between the basal membrane and "microscopic rough" concrete surface but no evidence of chemical adhesion between basal membrane and concrete can be observed. This observation indicates that the adhesion mechanism between basal membrane and concrete is attributed to physical adhesion to the microscopically rough surface.

Figure 4(c) shows the interfaces of attached area and detached area. The gap between basal membrane and concrete and some deposits are observed. Though it is likely shown that there is no basal membrane in the detached area, thin layer can be observed in the magnified image. Figure 6 shows the magnified image of detached area with distribution of elements. Ca-rich region of which thickness is $20 \mu \mathrm{m}$ can be observed near the concrete surface. The texture of the region is different from that of cement paste and is dense. This region would be produced by secondary organic-inorganic cement secreted by barnacles. Barnacles, which become partially or wholly detached from their substratum in a natural environment, produce a secondary cement secretion (Saroyan et al. 1970). Composition of the secondary secreted cement is similar to that originally secreted

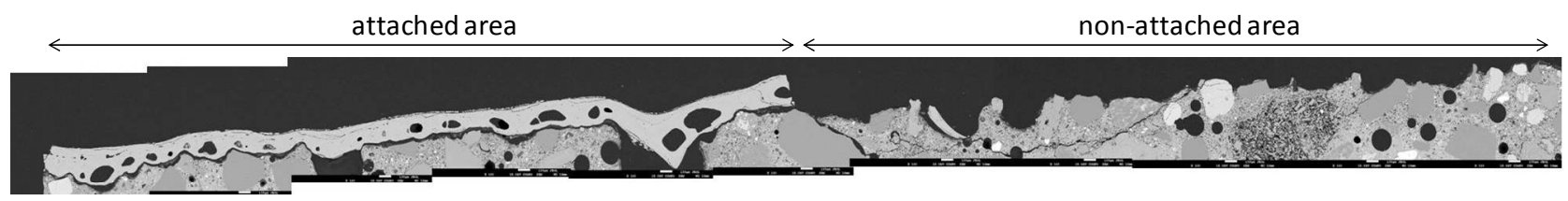

(a) Attached area and non-attached area (rough surface)

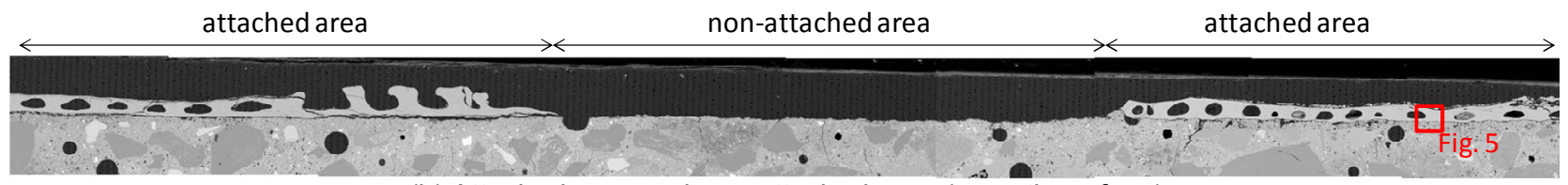

(b) Attached area and non-attached area (smooth surface)

non-attached area detached area attached area

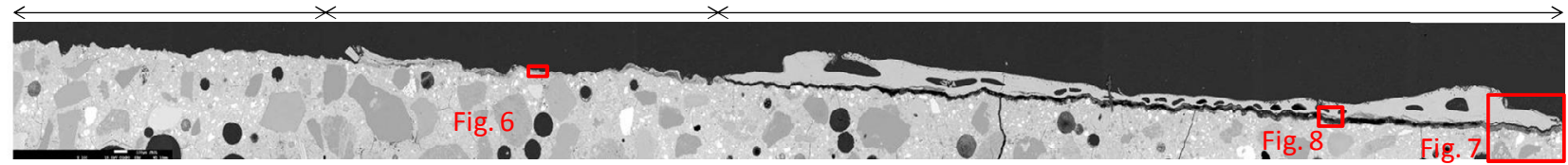

(c) Attached area and detached area

non-attached area attached area

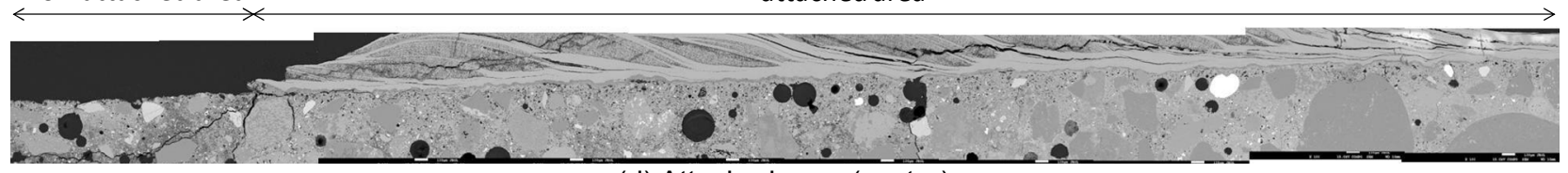

(d) Attached area (oyster)

Fig. $4 \mathrm{BEI}$ of interface between concrete and basal membrane (Type A). 


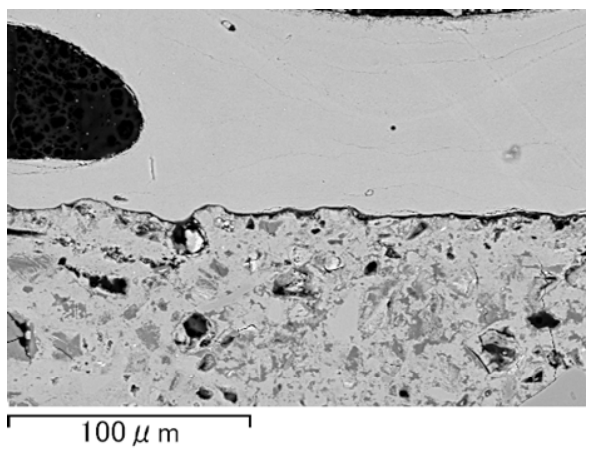

(a) BEI

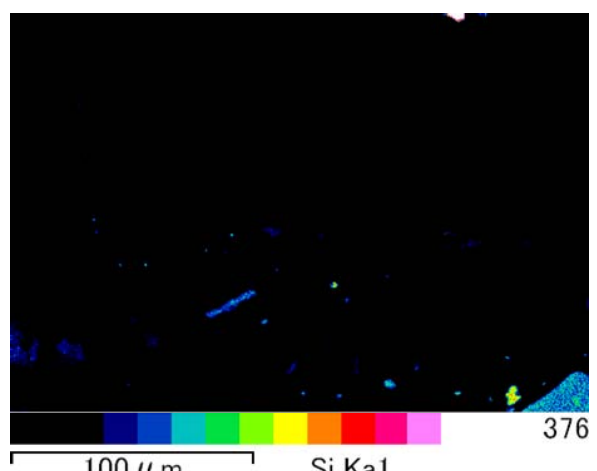

(c) $\mathrm{Si}$

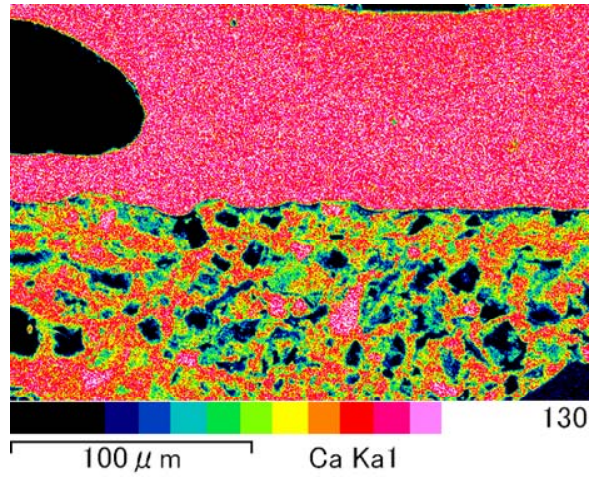

(b) $\mathrm{Ca}$

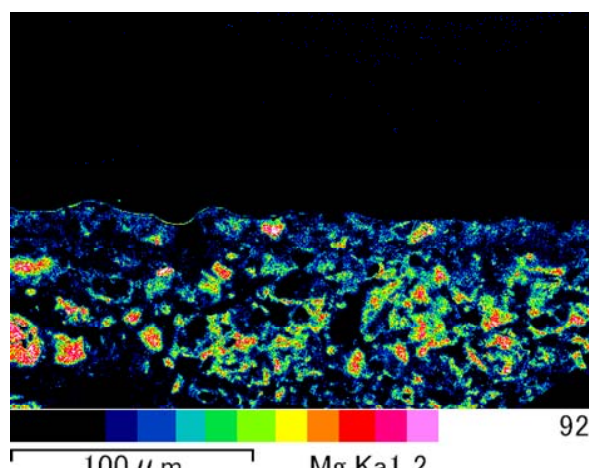

(d) $\mathrm{Mg}$

Fig. 5 Magnified image of attached area.

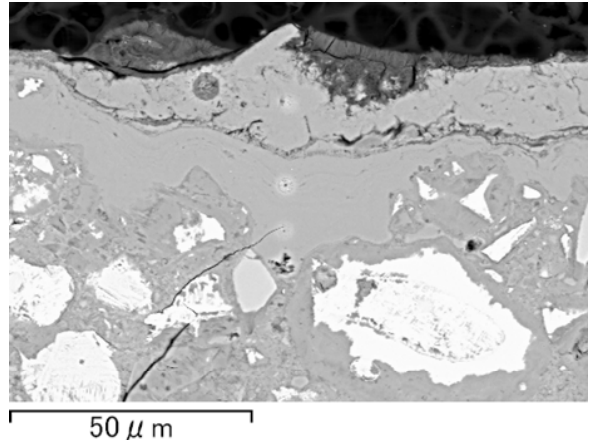

(a) BEI

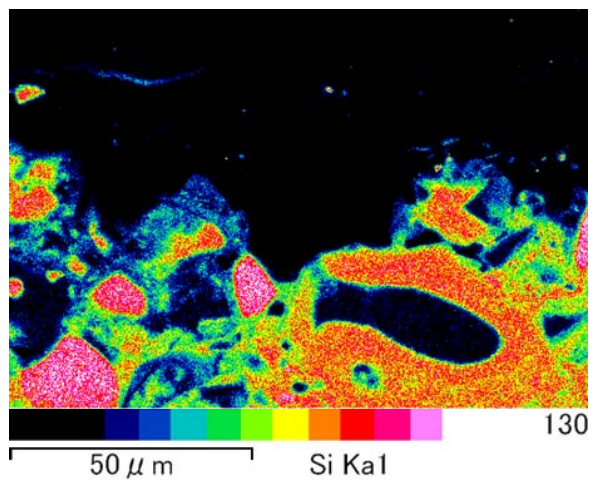

(c) $\mathrm{Si}$

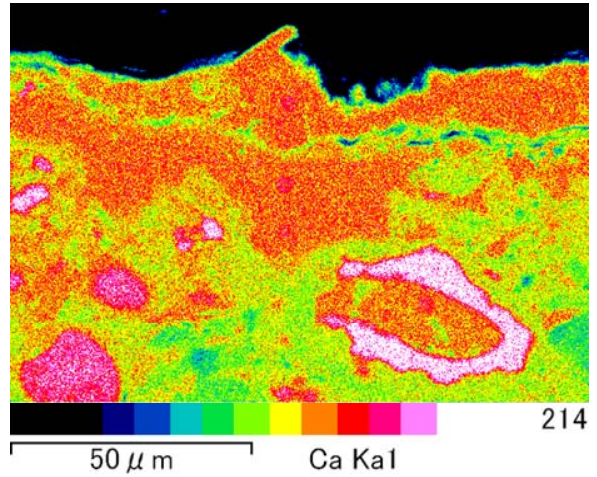

(b) $\mathrm{Ca}$

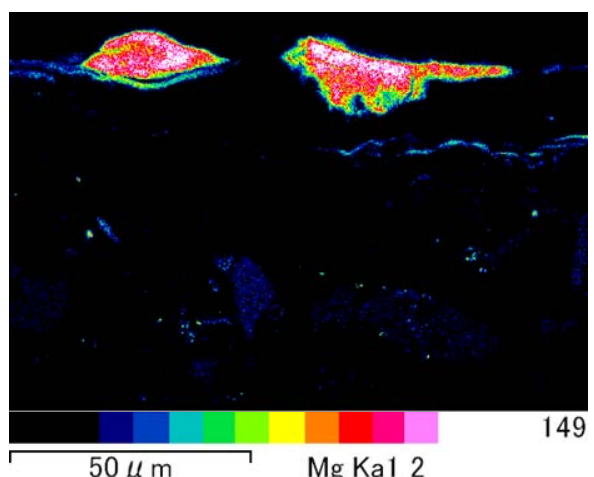

(d) $\mathrm{Mg}$

Fig. 6 Magnified image of detached area.

(Kamino et al. 1996).

In the gaps, as shown in Fig. 4(b) and Fig. 4(c), some deposits are observed. Figure 7 shows the magnified image of the boundary between attached and detached areas and distribution of elements. Mg-rich substances, which are supposed to be brucite, are precipitated in the 


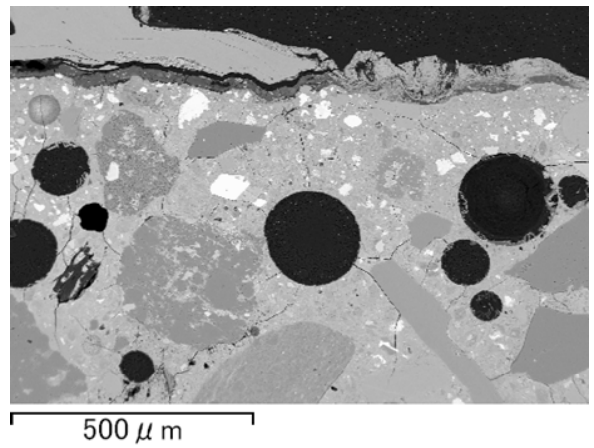

(a) BEI

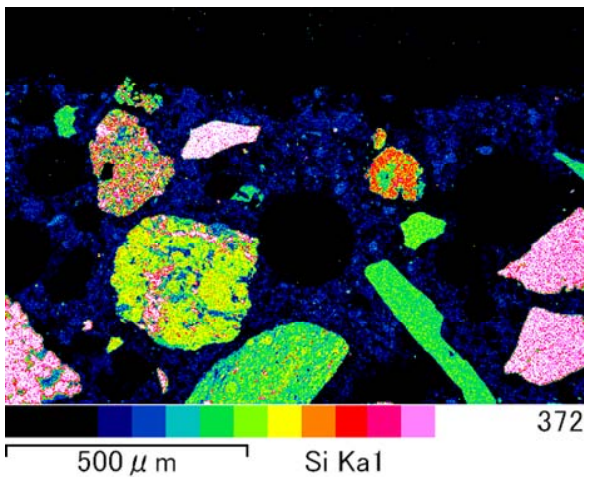

(c) $\mathrm{Si}$

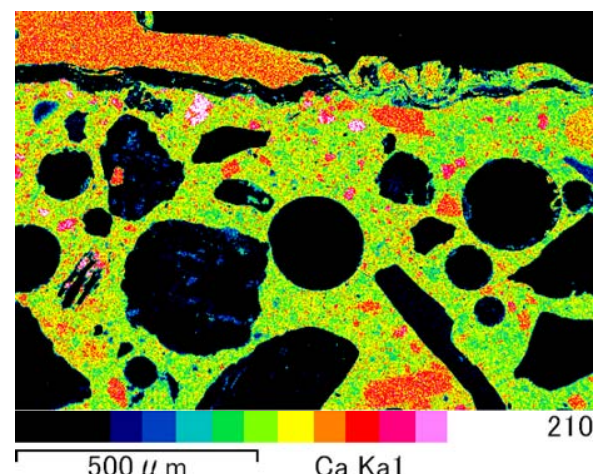

(b) $\mathrm{Ca}$

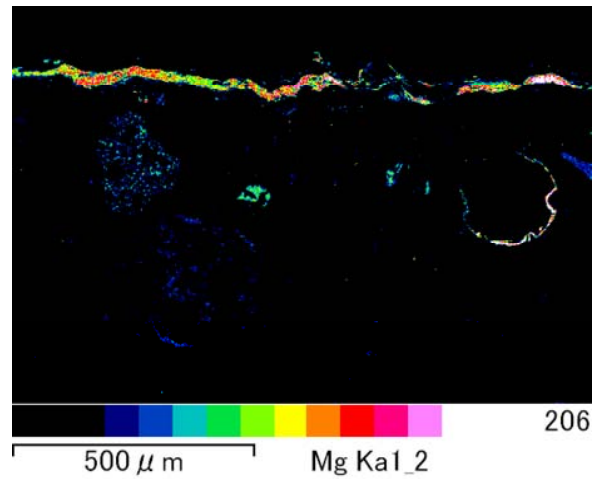

(d) $\mathrm{Mg}$

Fig. 7 Magnified image of gap.

gaps. The mechanism is attributed to localized increases in $\mathrm{pH}$ within the gap. From the solubility product of brucite, the higher $\mathrm{OH}^{-}$concentration is, the lower $\mathrm{Mg}^{2+}$. The seawater is saturated with respect to brucite at high $\mathrm{pH}$ (Buenfeld and Newman 1986). Figure 8 shows the magnified image of the gap. It is supposed that the basal membrane originally adheres to concrete well and the gap was not filled with brucite. This means that brucite has nothing to do with gap formation. The precipitation of brucite is not the cause of gap formation, but resulted from gaps derived from other factors.

Figure 4(d) shows the interface between concrete surface and oyster. It is obvious that oyster adheres to the concrete surface compared to barnacles. This observation implies that oysters can also inhibit chloride ion penetration.

\subsection{Electrical migration test}

Figure 9 shows the effective chloride ion diffusion coefficient, $D_{e}$, of concrete with marine sessile organisms. The mean $D_{e}$ of concrete without marine sessile organisms showed $0.53 \mathrm{~cm}^{2} /$ year. It should be noted that $D_{e}$ significantly decreased with an increasing attached area of marine sessile organisms. $D_{e}$ of concrete with $A_{a d}$ of $96 \%$ was only $10 \%$ of that without marine sessile organisms. This result suggests that attachment of marine sessile organisms on the concrete surface improves the resistance to chloride ion penetration.

In the experiment carried out in the previous research (Iwanami et al. 2002, Maruya et al. 2003), $D_{e}$ of 6 types

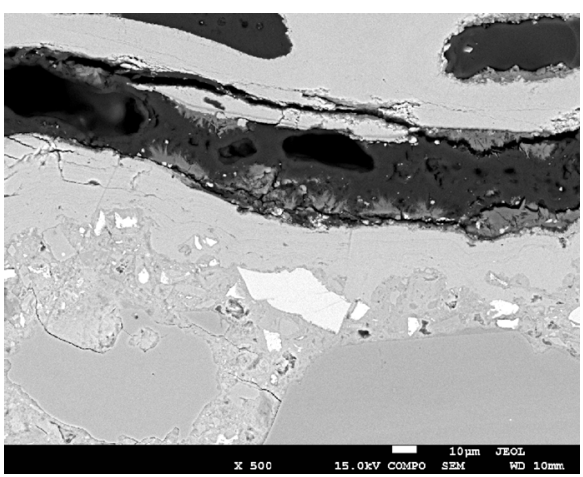

Fig. 8 Magnified image of gap.

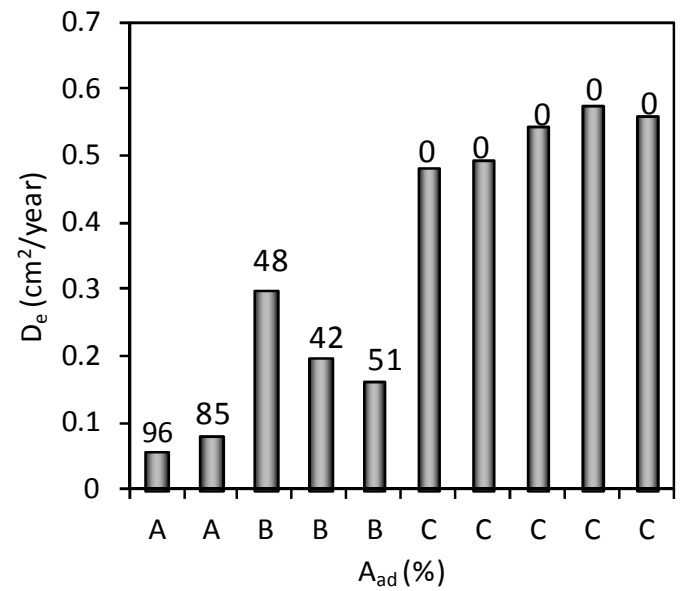

Fig. $9 D_{e}$ of concrete with/ without marine sessile organisms. 
of concrete with different materials and mixture proportions was evaluated. The experimental conditions are the same as that described in this paper except for the shape of the test specimen. The result showed that the attached area of marine sessile organisms strongly affects $D_{e}$ while the material properties and mixture proportions demonstrate no significant effect. Figure 10 shows the relationship between $A_{a d}$, attached and detached areas of marine sessile organisms, and the normalized $D_{e}$ of the concrete. The normalized $D_{e}$ is defined as the ratio of the $D_{e}$ of concrete to that of non-attached concrete. For comparison, the data published in the previous paper are plotted in this figure. In general, after about a 10 -year exposure, the trend between $A_{a d}$ and the normalized $D_{e}$ is almost the same as that published in the previous paper, irrespective of exposure conditions. Type B, in which the detachment between marine sessile organisms and concrete surface was accelerated, also showed the consistent trend. In fact, about more than half of the marine sessile organisms were detached from the concrete surface in Type B. It is interesting that the sum of the attached area and detached area is correlated to normalized $D_{e}$. This result suggests that not only the attached area but also the detached area inhibit chloride ion penetration on a long-term basis.

According to Wiegemann and Watermann (2004), barnacles cannot adhere to non-stick coatings when being desiccated. The drying process of a tidal zone, however, allows marine sessile organisms to be supplied with seawater or moisture from the concrete. The supply of water from concrete would help to keep the adhesion between marine sessile organisms and concrete surface. From these results, long-term resistance of concrete to chloride ion penetration can be promising in a natural marine tidal zone when an abundance of marine sessile organisms are attached on the concrete surface.

\subsection{Chloride ion content}

The chloride content profiles in concrete Type A with a different attached areas are shown in Fig. 11. The chloride content decreased with increase in the distance from the concrete surface. Figure 12 shows the total chloride ion content in concrete. The total chloride content penetrated into concrete with marine sessile organisms was less than half that of non-attached concrete. This result strongly supports that the attachment of marine sessile organisms prevents chloride ion from penetrating into concrete. In the tidal zone and submerged zone where the marine sessile organisms can attach continuously, high resistance of concrete to chloride ion penetration can be attained.

In order to obtain the apparent diffusion coefficient of concrete, the error function solution to Fick's second law was fitted to the chloride content profile in the concrete without marine sessile organisms, as shown in Eq. (1).

$$
C(x, t)=C_{0}\left(1-\operatorname{erf}\left(\frac{x}{2 \sqrt{D_{a} t}}\right)\right)
$$

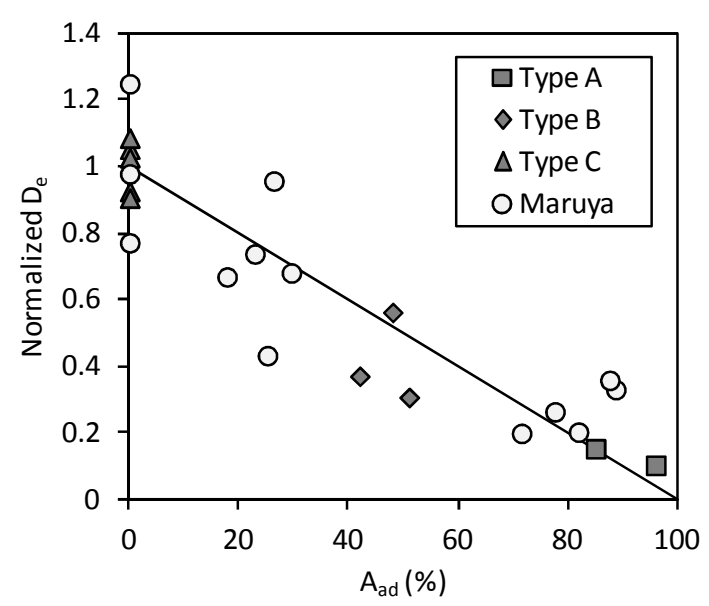

Fig. $10 A_{a d}$ and normalized $D_{e}$.

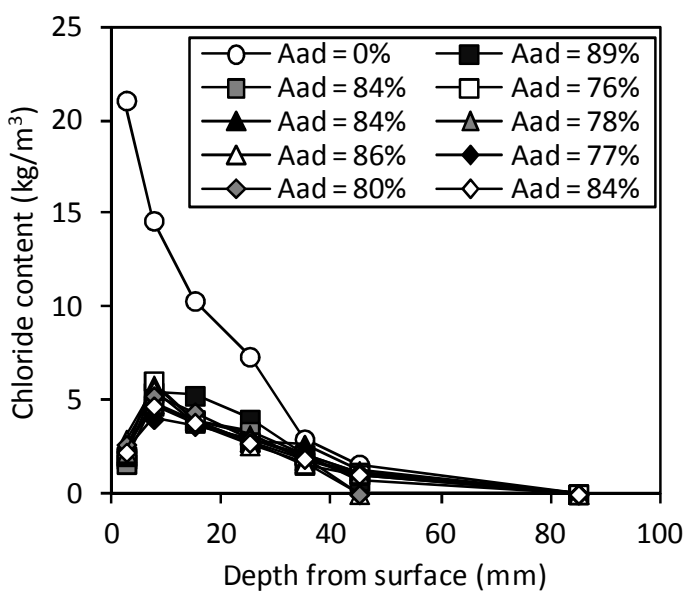

Fig. 11 Chloride content profiles of Type A concrete.

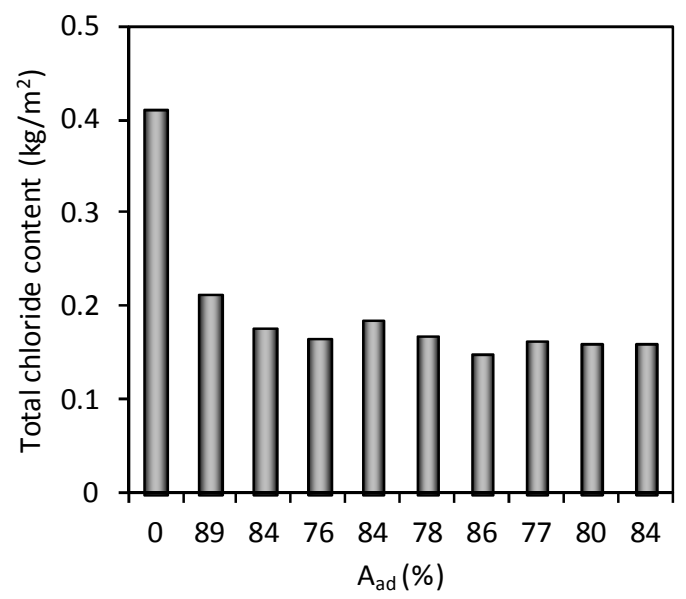

Fig. 12 Total chloride content in Type A concrete.

where, $C(x, t)=$ chloride content at depth $x$ and time $t, C_{0}$ $=$ chloride content at surface, $x=$ depth from concrete surface, $t=$ time, and $D_{a}=$ apparent diffusion coefficient. From the calculation, the apparent diffusion coefficient of concrete and chloride content at surface was 0.29 
$\mathrm{cm}^{2} /$ year, $21.5 \mathrm{~kg} / \mathrm{m}^{3}$, respectively. This calculation does not take into consideration time-dependent changes in either apparent diffusion coefficient or chloride content at surface.

Here, the effect of the marine sessile organisms on chloride ion diffusivity in concrete was considered. In the actual condition, marine sessile organisms attach continuously so that chloride ion penetration would decrease with the increase in attached area simultaneously. Simply assumed that the diffusion coefficient of basal membrane, $D_{m}$, is zero, chloride ion cannot penetrate from the attached area. This means the area where the chloride ion ingresses decreases with increase in attached area. Time-dependent change of attachment of marine sessile organisms is difficult to evaluate quantitatively as was attempted in the previous research (Maruya et al. 2003). For simplified evaluation, the time evolution of attachment of marine sessile organisms is expressed as in the following Eqs. (2) and (3).

$$
\begin{aligned}
& A_{a d(t)}=A_{a d \max } \times U_{(t)} \\
& U_{(t)}=\left\{\begin{array}{l}
1\left(t \geq t_{a}\right) \\
0\left(t<t_{a}\right)
\end{array}\right.
\end{aligned}
$$

where, $A_{\mathrm{ad}(t)}=$ the sum of attached and detached areas at time $t, A_{\text {admax }}=$ maximum value of $A_{\text {ad }}, U_{(t)}=$ step function, $t_{a}=$ the period at which $A_{(t)}$ equals to $A_{\text {admax }}$, and $t=$ time. According to Maruya et al. (2003), $A_{\text {ad }}$ reached the maximum value at the exposure period of 1 or 2 years so that $t_{a}$ was set to $0.5,1.5$ and 2 years. From the experiments, $A_{\text {admax }}$ was set to be $80 \%$. Before $t$ equals to $t_{a}$, the chloride ion diffusion was calculated according to Eq. (1). After $t_{a}$, the diffusion at attached and detached areas and non-attached area are calculated individually. In the attached and detached areas, the calculation method for surface coated concrete was used (Tsutsumi et al. 2010). Thereafter, the chloride content profiles are calculated with weighted average method. For simplified calculation, one dimensional diffusion was assumed. Table 3 summarizes the calculation condition.

Figure 13 shows the calculated results. Calculated chloride profiles show similar trends to experimental results. The difference between calculation and experiment at the concrete surface would be attributed to carbonation and Ca-leaching from concrete. It is also clear that the effect of $t_{a}$ on chloride profiles was small in this calculation. More importantly, the calculated results support that chloride ion had not been able to penetrate into concrete after 1 to 2 years of exposure. Assuming that such high resistance of concrete to chloride ion penetration will be sustained permanently, chloride ion content at $70 \mathrm{~mm}$ from the concrete surface, which is one of standard covering depths, can be calculated as 1.2 $\mathrm{kg} / \mathrm{m}^{3}$ after 50 -year service.

Chloride content profiles and calculations greatly suggest that the attachment of marine sessile organisms can enhance the long-term resistance of concrete to chloride ion penetration. In the previous paper, the ap-

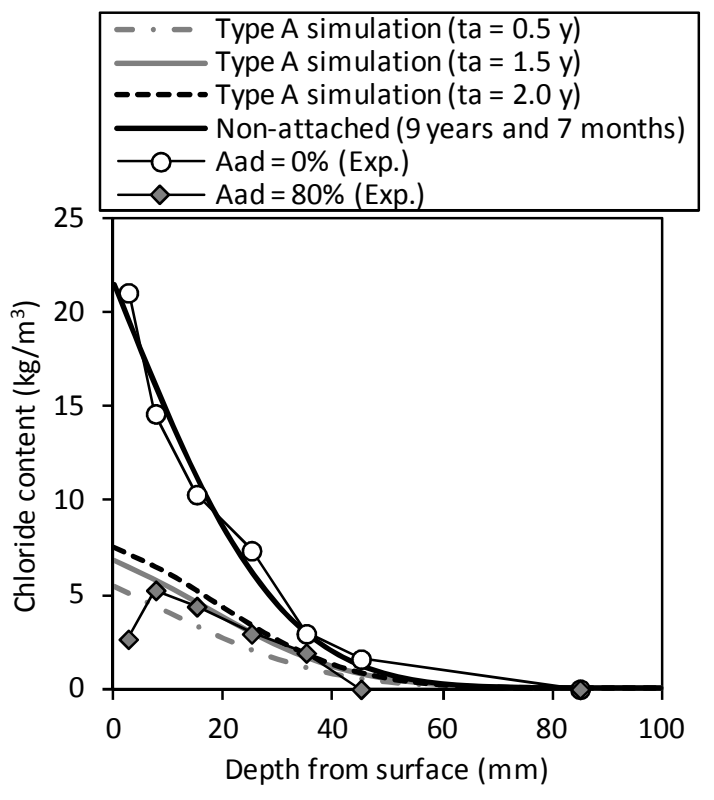

Fig. 13 Calculated chloride content profiles.

Table 3 Calculation condition.

\begin{tabular}{|c|c|}
\hline $\mathrm{C}_{0}\left(\mathrm{~kg} / \mathrm{m}^{3}\right)$ & 21.5 \\
\hline $\mathrm{D}_{\mathrm{a}}\left(\mathrm{cm}^{2} /\right.$ year $)$ & 0.29 \\
\hline $\mathrm{D}_{\mathrm{m}}\left(\mathrm{cm}^{2} /\right.$ year $)$ & 0 \\
\hline $\mathrm{t}_{\mathrm{a}}$ (year) & $0.5,1.5,2$ \\
\hline $\mathrm{A}_{\mathrm{admax}}(\%)$ & 80 \\
\hline
\end{tabular}

parent diffusion coefficient of concrete covered with marine sessile organisms is 100 times smaller than that of non-attached concrete. In the previous paper, however, the apparent diffusion coefficient was decided by exposure testing for 1 year and 8 months. It is well known that the apparent diffusion coefficient is a time-dependent parameter so the diffusion coefficient of marine sessile organisms would be a significantly smaller value, nearly equal to zero.

\subsection{Effect of attachment of marine sessile or- ganisms on chloride ion penetration}

From the results described above, the attachment of marine sessile organisms can enhance the long-term resistance of concrete structures to chloride ion penetration. Even if the marine sessile organisms are detached from the concrete surface, the secondary secreted cemented region would prevent the penetration of chloride ion. In existing marine structures, deterioration of concrete structures located in tidal and submerged zones is rarely more severe than our expectations. The result is well consistent with the empirical rule.

\section{Conclusions}

This paper investigated the long-term resistance of concrete with marine sessile organisms to chloride ion penetration. The exposure test spanned about 10 years and experiments were carried out. The conclusions can 
be summarized as follows:

(1) The basal membrane, which is a dense matrix of marine sessile organisms, adheres to concrete strongly on a long-term basis though some gaps between concrete and the basal membrane can be observed.

(2) In the detached area of the basal membrane, the region containing a high $\mathrm{Ca}$ content can be observed. This region would be produced by a secondary organic-inorganic cement secretion.

(3) The electrical migration test revealed that the sum of attached and detached areas of the basal membrane is well correlated to effective diffusion coefficient of concrete, irrespective of exposure duration. This result suggests that not only the attached area but also the detached area of marine sessile organisms can inhibit chloride ion penetration on a long-term basis.

(4) Total chloride ion content in concrete with marine sessile organisms was significantly less than non-attached concrete. Experimental results and simplified simulation clarified that the attachment of marine sessile organisms can enhance the long-term resistance of concrete to chloride ion penetration.

\section{References}

Buenfeld, N. R. and Newman, J. B., (1986). "The development and stability of surface layers on concrete exposed to sea-water." Cement and Concrete Research, 16, 721-732.

Gjorv, O. E., (1971). “ Long-time durability of concrete in seawater." ACI Journal, 68(10), 60-67.

Iwanami, M., Yokota, H., Hamada, H., Yamaji, T. and Watanabe, H. (2002). "Can marine fouling organisms extend the life of concrete structures?" Proceedings of the IABSE Symposium on Towards a Better Built Environment - Innovation, Sustainability, Information Technology, Paper No. PAP164 (CD-ROM).

Japan Society of Civil Engineers, (2005). "JSCE Guidelines for Concrete No.2." Japan Society of Civil Engineers.

Kamino, K., Odo, S. and Maruyama, T., (1996). "Cement proteins of the acorn barnacles, Megabalanus rosa." Biological Bulletin, 190, 403-409.

Maruya, T., Iwanami, M., Sakai, E., Mashimo, M. and Hamada, H., (2003). "Durability enhancement of RC structures covered with a dense layer formed by marine aquatic fouling organisms." Journal of Materials, Concrete Structures and Pavements, 739(60), 61-74. (in Japanese)

Nilsson, L.-O., (2009). "Models for chloride ingress into concrete - from Collepardi to today." International Journal of Modelling, Identification and Control, 7, 129-134.

Port and Airport Research Institute, (2007). "Manual on maintenance of Port and Harbour Facilities." Coastal Development Institute of Technology. (in Japanese)

The sessile organisms society of Japan, (2006). "Latest topics on barnacles." Kouseisha-kouseikaku Corporation. (in Japanese)

Tsutsumi, T., Onitsuka, S., Seshimo, Y., Nakagawa, T. and Iwanami, M., (2010). "Research on quantitative evaluation of effect of repair to reinforced concrete damaged by chloride attack." Proceedings of 4th ACF International Conference, Paper No. PO-09 (CD-ROM).

Wiegemann, M. and Watermann, B., (2004). "The impact of desiccation on the adhesion of barnacles attached to non-stick coatings." Biofouling, 20, 147-153. 


\title{
Development of Engineered Self-Healing and Self-Repairing Concrete- State-of-the-Art Report
}

\author{
Hirozo Mihashi ${ }^{1}$ and Tomoya Nishiwaki ${ }^{2}$
}

\begin{abstract}
Challenging studies of engineered self-healing and self-repairing functions in concrete structures are briefly reviewed. While self-healing of concrete has been studied for a long time, it was only recently noticed that some engineered technologies are useful to stimulate the potential of concrete to be self-healed. For example, fiber reinforced cementitious composites (FRCC) have a much higher potential of self-healing than ordinary concrete because of their capability to keep cracks thinner and also because of the bridging network system in cracks; a specific bio-chemical approach, i.e. the application of mineral-precipitating bacteria, is now available; and various mineral admixtures are useful for practical application. Furthermore, the new concept of self-repairing concrete, which is based on the design concept of intelligent materials, is reported. Self-repairing concrete is concrete that incorporates devices for achieving the three key functions of an intelligent material, (1) sensing, (2) processing, and (3) actuating.

This paper is a state-of-the-art report on the recent development of engineered self-healing and self-repairing concrete.
\end{abstract}

\section{Introduction}

Concrete structures often suffer from cracking that leads to much earlier deterioration than designed service life. To prevent such deterioration, regular inspection of cracks in concrete structures and their repair are usually carried out by means of some kind of human intervention. On the other hand, for example, a small cut on our body can be healed by a simple treatment even though it takes a couple of days. In nature, animals and trees usually can heal small bodily damage by themselves.

Generally speaking, cracks in concrete can occur in any stage of the service life of concrete structures due to volume instabilities such as autogenous shrinkage and/or drying shrinkage since concrete is composed of aggregate of various sizes connected with the hydration products generated by mixing cement and water. Furthermore, the tensile strength of concrete is about $10 \%$ of the compressive strength, so that concrete is reinforced with steel bars at least in the parts subject to tensile stress. Once cracking occurs in reinforced concrete members, not only is the stiffness reduced but steel bars corrosion also occurs due to the permeation of rain and aggressive substances, reducing structural safety and serviceability. In order to avoid the dangerous situations caused by such deterioration, application of proper maintenance systems is required. For concrete structures to avoid most such damage, the initial performance of concrete needs to be set at a high level and comprehensive maintenance sys-

${ }^{1}$ Professor, Tohoku Institute of Technology, Sendai, Japan.

E-mail: mihashi@tohtech.ac.jp

${ }^{2}$ Tohoku University, Graduated School of Engineering, Sendai, Japan. tems need to be applied. This presents the drawback of higher construction cost.

In the meantime, sustainability is now one of the top issues in the field of building and civil engineering from the viewpoint of global ecology. For this reason, extending the service life of structures has become a key objective. A full array of inspection and maintenance techniques for concrete structures has been developed. In some cases, however, it is difficult for engineers to access damaged sites for repair work because of their location and/or environmental conditions. Some examples are underground structural members, radioactive waste disposal facilities, and walls of tanks storing highly toxic waste.

The availability of self-healing and self-repairing systems would make structures more reliable. For example, if control and repair of early-stage cracks in concrete structures were possible, permeation of driving factors for deterioration could be prevented, thus extending the service life of the structures. For this reason, many papers have been published on self-healing and self-repairing concrete

Overcoming the apparent contradictory requirements of low cost and high performance is a challenging task. A major goal of concrete technologies might be to make concrete a functional material to meet a specific set of performance requirements. The adaptation of new technologies to structural engineering is expected to result in better, stronger, and more durable structures at lower life cycle costs. Meanwhile, the development of advanced concrete materials should be related to the cost-effective engineering means to achieve the desired structural properties.

In recent years, intelligent materials have been extensively developed in various research fields using a new material design approach based on the concept of in- 
stalling smart functions such as sensing, processing and actuating in the material itself (Science and Technology Agency 1989). Shahinpoor (1997) reported that intelligent materials are currently defined as materials capable of automatically and inherently sensing or detecting changes in their environmental conditions and responding to those changes with some kind of actuation or action. Once the sensing function catches a change, the influence on the required performance of the structure is assessed. If the influence is not negligible, the change is repaired or improved automatically. In order to accomplish such functions, advanced apparatus such as optical fiber sensors, shape memory alloy and piezoelectric devices are usually employed. Generally speaking, the merging of such advanced devices results in a substantial escalation in costs. In specific fields such as aerospace and medical engineering, reliability and performance are the dominant factors in material design and cost is a secondary consideration. In the field of concrete used in civil and building engineering, however, solutions that involve a steep rise of the cost of materials are hard to justify in terms of the cost-benefit relation. Hence, the viability of new materials in the field of concrete engineering is highly controlled by their cost.

In this report, previous papers related to the self-healing and self-repairing concrete are briefly reviewed.

\section{Definition of terms - self-healing and self-repairing}

Self-healing, or autogenous healing, of concrete and reinforced concrete is a phenomenon that has been studied by various researchers. Reviews on healing of cracks can be found for example in Lauer \& Slate (1956), Jacobsen et al. (1998) and de Rooij \& Schlangen (2011). Many experimental results and practical experiences have demonstrated that cracks in concrete have the ability to heal themselves, reducing water flow through cracks over time. According to a review of the literature by Lauer \& Slate (1956), the action of self-healing was first discovered by the French Academy of Science in 1836, which concluded that self-healing is the conversion of calcium hydroxide exuded from the hydrated cement and converted to calcium carbonate on exposure to the atmosphere. Many subsequent researchers, however, assumed that self-healing is an action of continued hydration and other actions. Summarizing previous studies, possible mechanisms of self-healing are cited as follows (Ramm and Biscoping 1998): (1) further reaction of the unhydrated cement; (2) expansion of the concrete in the crack flanks; (3) crystallization of calcium carbonate; (4) closing of the cracks by solid matter in the water; (5) closing of the cracks by spalling-off of loose concrete particles resulting from the cracking. Among these five mechanisms, however, Edvardsen (1999) clarified that crystallization of calcium carbonate within the crack is the main mechanism for self-healing of ma-

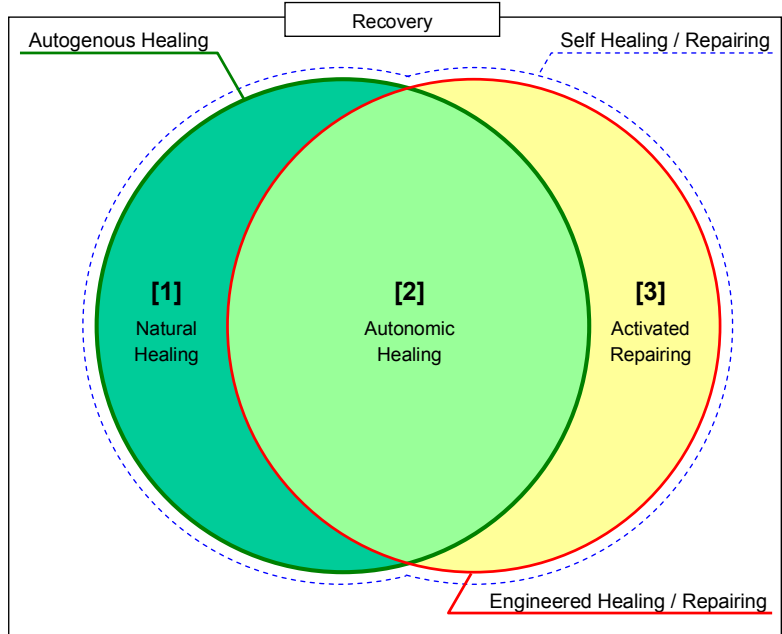

(a) Definition of self-healing/repairing concrete (JCI 2009, Igarashi et al. 2009).

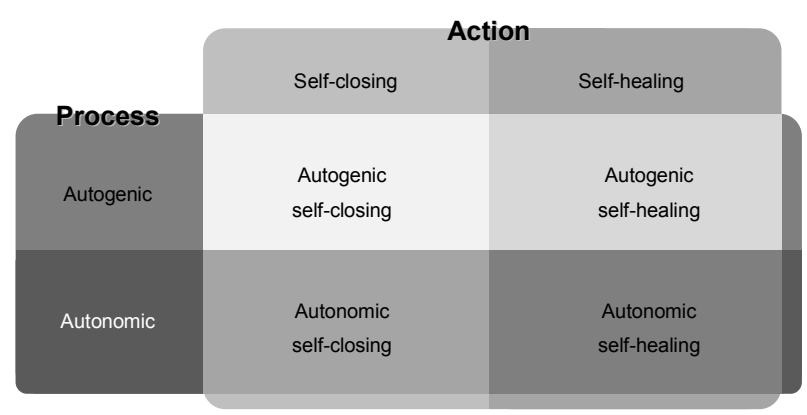

(b) Definition of self-healing concrete based on the action and the process by RILEM-TC221 (de Rooij \& Schlangen 2011).

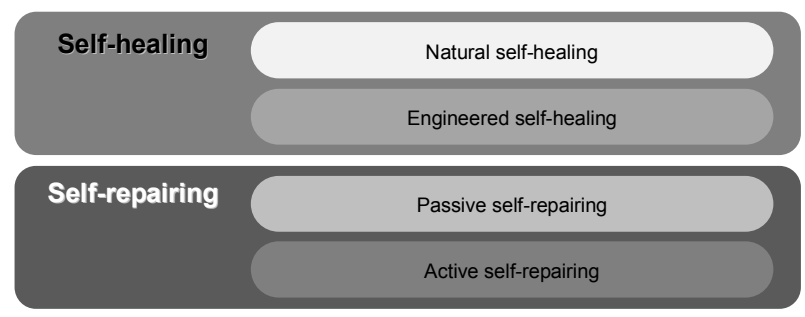

(c) Definition of self-healing/repairing concrete by the authors.

Fig. 1 Definition of Self-Healing and Self-Repairing Concrete

tured concrete.

While various techniques for engineered healing or repairing of cracks in concrete have been proposed so far, the targeted crack widths and adopted techniques greatly differ depending on the objective. For example, strength should be recovered for structural safety performance, but for durability performance, just filling cracks to prevent the permeation of water and aggressive substances is sufficient. Moreover, the key points are the materials and mechanisms used for filling up the cracks.

JCI Technical Committee on Autogenous Healing in 
Cementitious Materials (JCI 2009) proposed the following definition of self-healing/repairing concrete: (1) Natural healing; (2) Autonomic healing; and (3) Activated repairing. Furthermore, (4) Autogenous healing covers natural healing and autonomic healing (i.e. 1+2), and (5) Engineered healing/repairing covers autonomic healing and activated repairing (i.e. 2+3). Finally Self-Healing/Repairing covers all the actions of closing and/or repairing cracks (Fig. 1 (a)).

On the other hand, in RILEM Technical Committee 221-SHC: Self-healing phenomena in cement-based materials, self-healing terms are defined based on the result of the action: "self-closing" or "self-healing"; and on the process of the action: "autogenic" or "autonomic" (de Rooij \& Schlangen 2011). Thus they are subdivided into the following four groups: (1) Autogenic self-closing: own generic material closes cracks; (2) Autogenic self-healing: own generic material restores properties; (3) Autonomic self-closing: engineered additions close cracks; and (4) Autonomic self-healing: engineered additions restore properties (Fig. 1 (b)).

Roughly speaking, previous studies on the subject can be divided into two groups: the first group focuses on the potential retaining capability in concrete (or cementitious composites) to fill cracks and some engineered technologies are installed to stimulate that capability; the second group opts to supplement a function to repair cracks, and some devices are embedded in advance for that purpose. In this report, the former approach is called "engineered self-healing" and the latter is called "self-repairing." The latter can be further subdivided into two sub-groups: one is passive mode self-repairing in which functional elements such as hollow pipes are embedded in the designed position of the structural member similarly to reinforcing steel bars; the other is active mode self-repairing in which cracking is monitored by a sensor and cracks are repaired by actuation devices only when they become wider than a critical width (Fig. 1 (c)).

\section{Natural self-healing}

Lauer and Slate (1956) reported a study of the nature of the self-healing action and of the increases in tensile strength measured perpendicular to the plane of the crack. They studied the influence of age, conditions of curing, additions of lime and fly ash, and cycles of wetting and drying on healing. For example, Fig. 2 shows an increase in healing with higher water-cement ratios, in which 1-90 days means the initial breaking age was 1 day and then curing was done for 90 days until the testing of the healing effect. On the basis of the results of microscopic examination, they concluded that the bonding materials formed during the action were $100 \%$ calcium carbonate $\mathrm{CaCO}_{3}$ and calcium hydroxide $\mathrm{Ca}(\mathrm{OH})_{2}$ crystals but that no amorphous hydrated products of cement were found, though the test samples were young (1 to 28 days). Their results indicated that the addition of lime and fly ash as

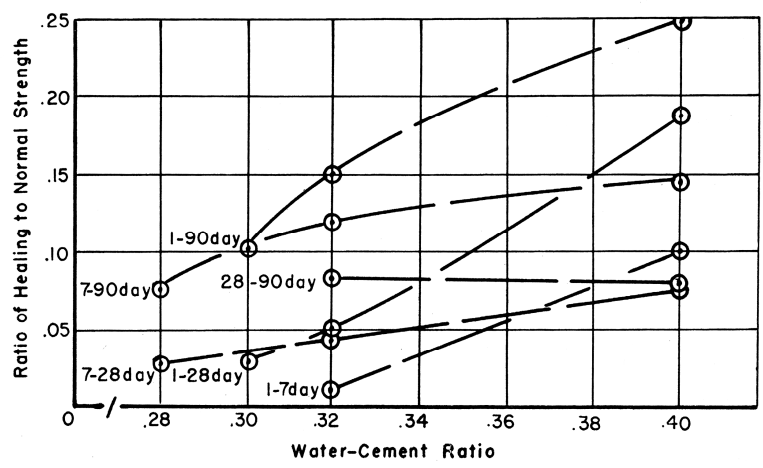

Fig. 2 Plot of ratio of healing to normal strength versus water-cement ratio (Lauer and Slate, 1956).

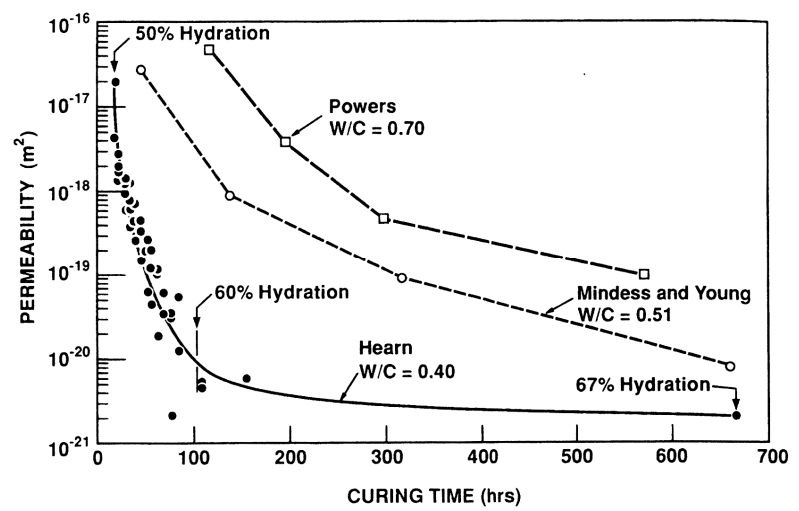

Fig. 3 Permeability vs. extent of hydration (Hearn and Morley 1997).

mixtures had a slight detrimental effect on the subsequent healing strength in water curing but that no definite trend was found in an atmosphere of $95 \%$ relative humidity. They emphasized that the presence of water as the curing medium was essential to obtain the maximum healing strength.

Dhir et al. (1973) studied the influence of age and mix proportions on the self-healing of mortars. They concluded that the rate of healing decreased with age within the test range of 7-120 days and that the percentage of recovery in strength was greater for the mixes with higher cement contents. They also concluded that the ultimate strain of healed specimens was reduced.

Hearn and Marley (1997) carried out a permeability test with 26 years old concrete and confirmed that drying and re-saturation resulted in a substantial increase in the self-healing effect and that extensive microcracking due to drying stimulated the effect (Fig. 3) since most of the flow took place through cracks exposed to the atmosphere. Furthermore, he suggested that the reduction in permeability due to hydration had a minimal effect, as shown in Fig. 4 (Hearn 1998).

Ramm and Biscoping (1998) performed an experimental study over a period of two years with respect to the self-healing and reinforcement corrosion of water-penetrated separation cracks in reinforced concrete in 

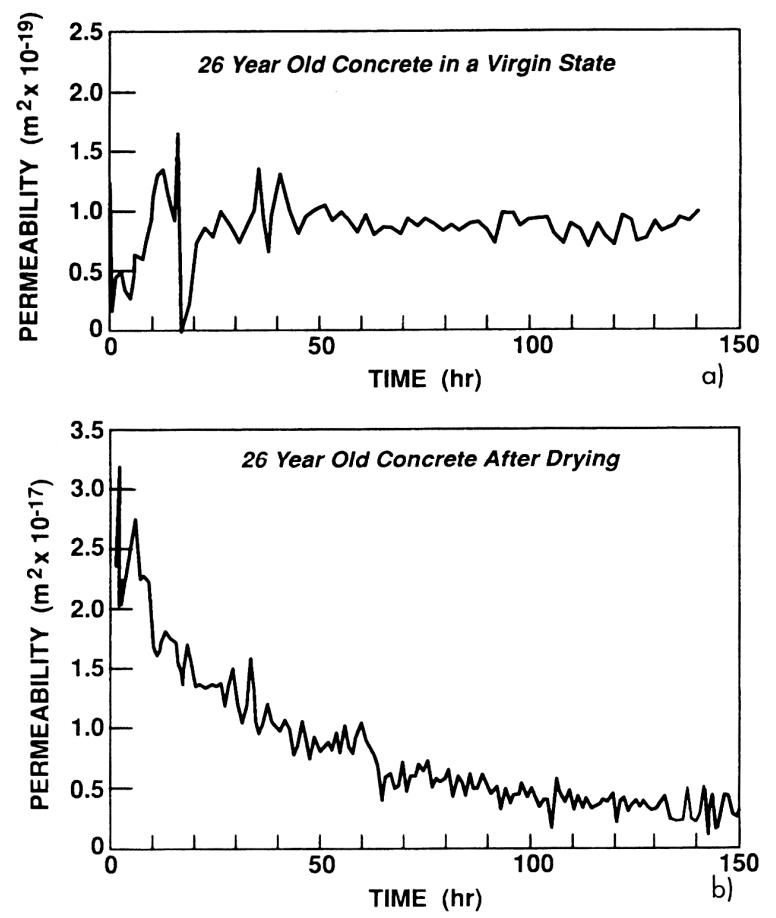

Fig. 4 Typical permeability vs. time data for virgin (a) and oven dry/resaturated (b) concrete (Hearn 1998).

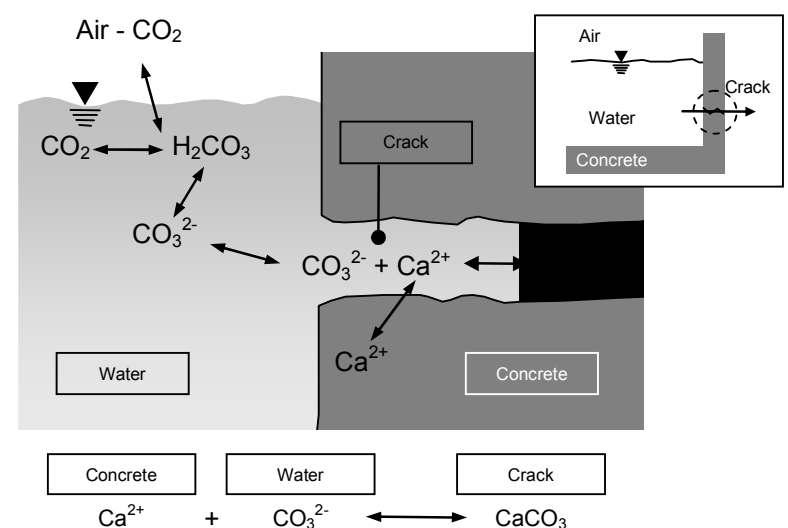

Fig. 5 Media, interfaces, and reactions in $\mathrm{CaCO}_{3}-\mathrm{CO}_{2}-\mathrm{H}_{2} \mathrm{O}$ system (Edvardsen 1999).

which the influence of the crack width, the thickness of the structure, the water pressure, and the degree of acid of the water were studied. The following conclusions were obtained. With a crack width of $0.1 \mathrm{~mm}$, corrosion was not observed in any case. For the test specimens with a crack width of $0.2 \mathrm{~mm}$, corrosion was found to start depending on the $\mathrm{pH}$ value. At increasing crack widths, increasing corrosion development is to be expected for test specimens penetrated by acid water. At the crack width of $0.4 \mathrm{~mm}$ and $\mathrm{pH}$ value of 5.2, the highest corrosion development was observed. However, there was no weakening of the cross section worth mentioning even at the end of the 2-year test period.

Edvardsen (1999) carried out a series of permeability tests on plain concrete and investigated the effects of self-healing upon the leakage of water through cracks in concrete. He found that the greatest self-healing effect occurred within the first 3 to 5 days of water exposure and that the precipitation of $\mathrm{CaCO}_{3}$ crystals in the cracks was practically the sole cause for the self-healing of the cracks. The growth rate of the $\mathrm{CaCO}_{3}$ crystals was dependent on crack width and water pressure, whereas the concrete composition and type of water had no influence on the self-healing rate. Edvardsen also concluded that the formation of $\mathrm{CaCO}_{3}$ responded to two different crystal growth processes. In the initial phase of water exposure, the kinetics of crystal growth is a surface-controlled crystal growth but later this changes to a diffusion-controlled crystal growth (Fig. 5). A similar mechanism of healing was also suggested by Lauer and Slate (1956).

Reinhardt and Jooss (2003) reported a series of permeability tests with the constant temperature at 20, 50, and $80^{\circ} \mathrm{C}$. They showed that the decrease of the flow rate depends on crack width and temperature and that a higher temperature favors a faster self-healing process.

Granger et al. (2007) carried out an experimental study on the mechanical properties of ultra high performance concrete and concluded that the self-healing of the pre-existing cracks was mainly due to hydration of anhydrous clinker on the crack surface and that the stiffness of newly formed crystals is close to that of primary C-S-H.

As for the recovery of material properties due to self-healing, Aldea et al. (2000) reported that the recovery in signal transmission with crack healing was not as spectacular as that in permeability.

The effect of self-healing on the properties of concrete subjected to cycles of freezing and thawing has been suggested as early as the middle of the 20th century (McHenry \& Brewer 1945, Lauer \& Slate 1956). Jacobsen et al. $(1995,1996)$ studied the microcracking of concrete due to freeze/thaw (1 to 10 micrometers) and self-healing of cracks after subsequent storage of deteriorated specimens in water for three months, using a scanning electron microscope (SEM). Two concrete types of $\mathrm{W} / \mathrm{B}=0.4$ and $\mathrm{SF} / \mathrm{B}=0$ or 0.05 were investigated. Deterioration/healing was measured by resonance frequency (dynamic modulus of elasticity) and compressive strength. While freezing/thawing led to substantial loss in both resonance frequency and compressive strength, subsequent self-healing gave a substantial recovery of the frequency but only a small recovery in the compressive strength. After self-healing, solid hydration products bridging cracks smaller than 5 micrometer were observed at several locations. Occurrence of the products was less apparent in the concrete with SF than in OPC concrete. Energy dispersive $\mathrm{X}$-ray analysis revealed that the composition of the products was of the C-S-H type. Calcium hydroxide crystals and ettringite were observed, too. 


\section{Engineered self-healing of concrete}

\subsection{Engineering with fiber reinforcement}

Compared with the rather large number of studies of self-healing of plain concrete, such studies on Fiber Reinforced Cementitious Composites (FRCC) are rather scarce. Hannant \& Keer (1983) carried out an experimental study on the recovery of elastic modulus and tensile strength of thin FRCC sheets under tension. W/C was 0.34 using OPC mortar containing fine silica sand of 150 to 300 micrometers and reinforced with a polypropylene network. The specimens were $1.2 \mathrm{~mm}$ in thickness and $30 \mathrm{~mm}$ in width. The study concluded that specimens containing about 22 cracks of the average width of 7 micrometers showed almost complete recovery of elastic modulus but only about $50 \%$ recovery of tensile strength after curing of seven months to two years in natural weathering conditions.

Grey (1984) examined self-healing of the interfacial bond strength between steel fiber and mortar by means of a pulling-out test on single fibers embedded in water-cured specimens. The test results indicated that the extent of the interfacial bond healing was greater than that observed for the compressive strength of the plain mortar.

Li et al. (1998) carried out experimental studies on the self-healing capability of Engineered Cementitious Composites (ECC) and concluded that cementitious materials with inherently tight crack widths are conducive to self-healing and that self-healing can distinctly recover the stiffness of cracked ECC, a fact established through resonance frequency measurement.

Yang et al. (2005) investigated self-healing of ECC subjected to wetting and drying cycles. They used a mix proportion of water-binder ratio of 0.25 including a large amount of fly ash and 2\% PVA fiber by volume fraction. The ECC material subjected to tensile load exhibited multiple cracking in which the crack width was between 60 and 80 micrometers on average. It was found that the resonance frequency could recover $76 \%$ to $100 \%$ of the initial value and that the tensile strain after self-healing could recover $1.8 \%$ to $3.1 \%$ for specimens pre-loaded to high levels of strain between $2 \%$ and 3\%. Yang et al. (2009) also reported similar results and showed that the majority of the self-healed products were characteristic of calcium carbonate crystals (Fig. 6).

Herbert \& Li (2011) reported the results of an experimental study on the self-healing behavior of ECC under natural environmental conditions. They concluded that self-healing was not as robust as that observed in some experimental results carried out under controlled laboratory conditions, though the self-healing in the natural environment was promising.

Homma et al. (2008, 2009) investigated the self-healing capability of FRCC by microscope observation, water permeability test, tension test and backscattered electron image analysis. They prepared specimens with water/binder ratio of 0.45 , containing three different types of fiber (i.e. steel cord 0.75 vol.\%: SC, polyethylene fiber 1.5 vol.\%: PE, and hybrid type including both of these two fibers: 0.75 vol. $\%$ \& 0.75 vol. $\%$ for a total of 1.5 vol. $\%$ : PE+SC). It was found that lots of very fine PE fibers bridge over the crack and that crystallization products of calcium carbonate examined with Raman spectroscopy become easily attached to the PE fibers. As a result, in the case of PE, the mean thickness of the crystallization products attached to the crack surface increased much faster compared with the other fibers (Fig. 7). Water permeability reduced as a function of the crack width together with the curing time for the self-healing, though the reduction rate was not improved for crack widths greater than 100 micrometers even if 1.5 vol.\% of PE was contained (Fig. 8). While specimens of $\mathrm{PE}+\mathrm{SC}$ showed a significant improvement in tensile strength, the improvement in specimens of $\mathrm{PE}$ was only $10 \%$ to $60 \%$, although the thickness of the attached crystallization products was the largest (Figs. 9 \& 10). Furthermore, by means of backscattered electron

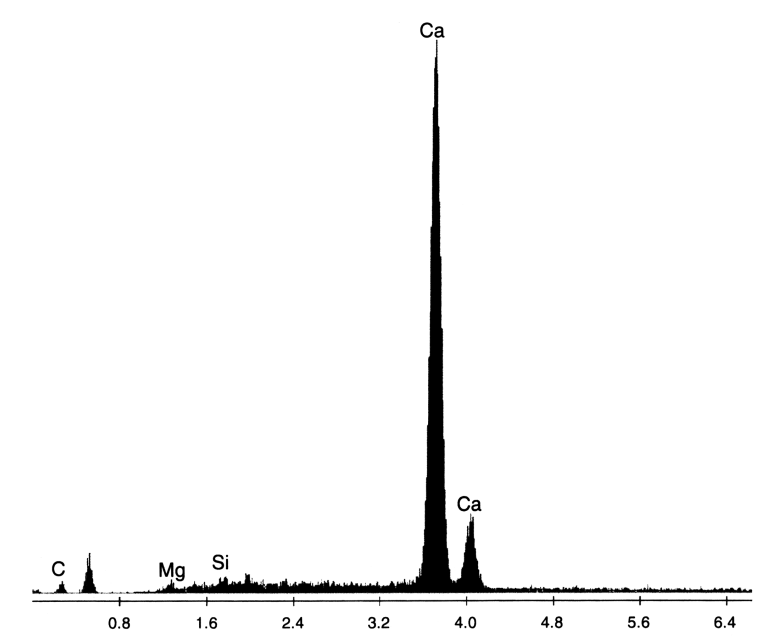

Fig. 6 Chemical composition of self-healed products (Yang et al. 2009).

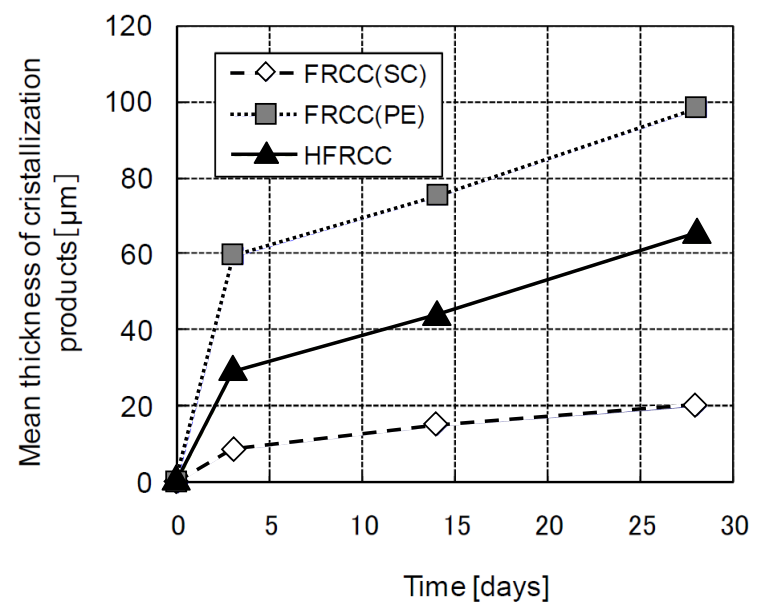

Fig. 7 Time dependence of mean thickness of crystallization products attached to the crack surface (Homma et al. 2009). 


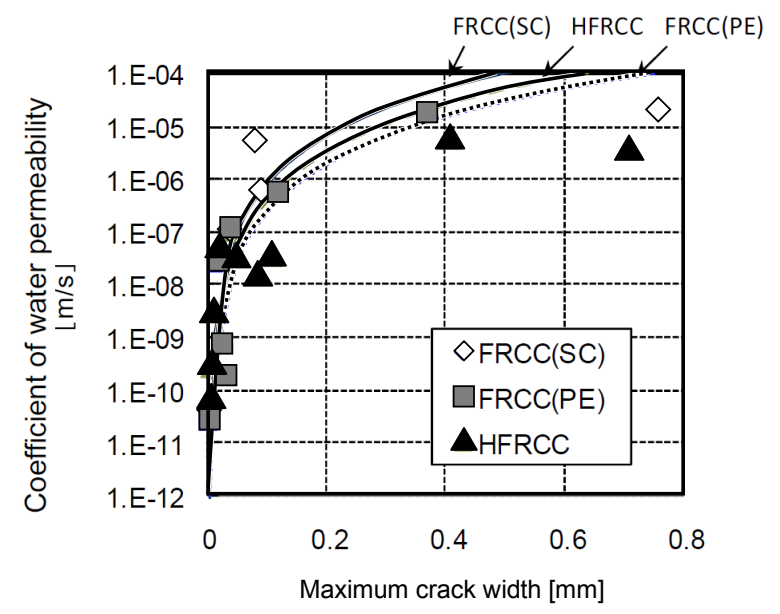

Fig. 8 Relationship between the coefficient of water permeability and the maximum crack width (Homma et al. 2009).

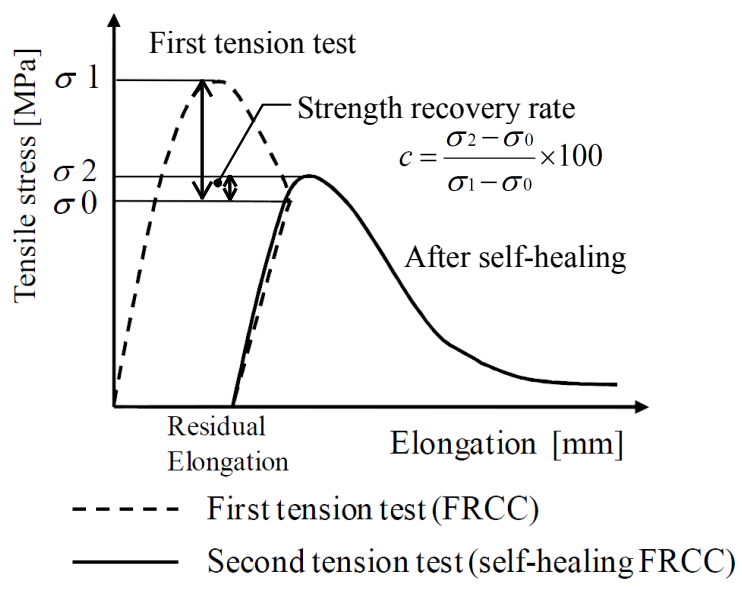

Fig. 9 Schematic description of the relationship between tensile stress and tensile elongation of FRCC (Homma et al. 2009).

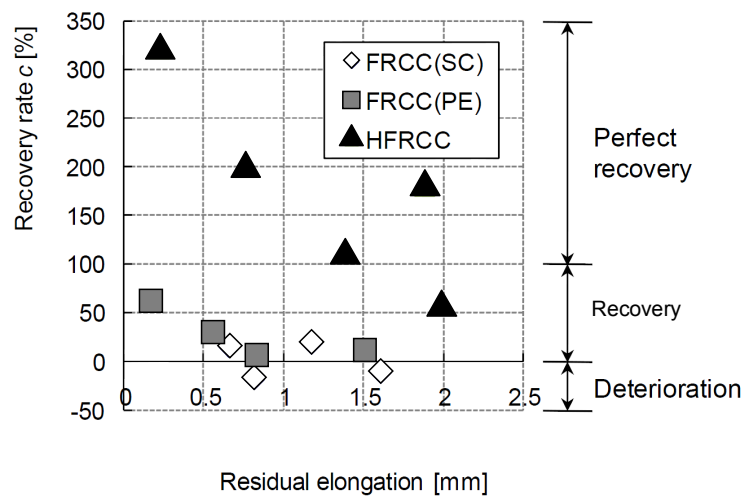

Fig. 10 Relationship between recovery rate and residual elongation (Homma et al. 2009).

image analysis, it was also verified that the difference of hydration degree had little influence on the self-healing capability.

Koda et al. (2011) carried out a similar permeability test to that of Homma et al. (2009) using PE and PVA

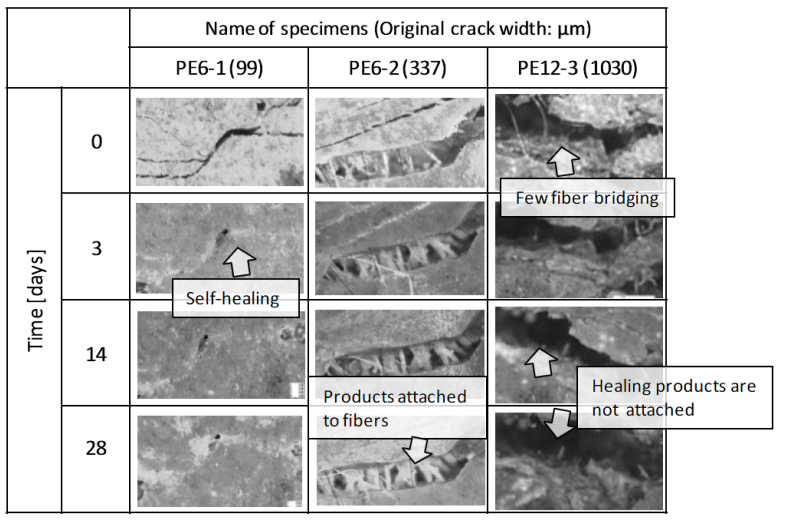

(a) PE series

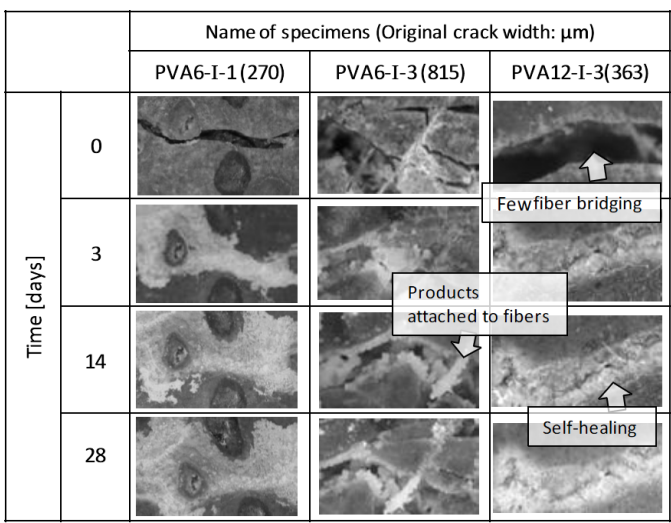

(b) PVA series

Fig. 11 Microscopic observation of crystallization products on crack surface (Koda et al. 2011).

fibers in which the volume content of both fibers was $1.5 \%$. Fibers such as PVA fiber that possess chemical polarity had a significantly higher capability for self-healing of cracks caused by pre-loading (Fig. 11). It is noteworthy that the capability of self-healing of PE and PVA is almost the same for cracks thinner than 100 micrometers, but the difference becomes significant for cracks wider than 100 micrometers (Fig. 12).

Self-healing of FRCC has also been noticed as a key factor that contributes to the significant reduction of steel bar corrosion in RC members. Sanjuan et al. (1997) reported that FRCC containing only 0.5 vol.\% of polypropylene with $\mathrm{W} / \mathrm{C}=0.5$ demonstrated an ability for self-healing of cracks under a corrosive environment.

Mihashi et al. (2011) reported the results of a series of long-term corrosion tests on FRCC containing PE alone and hybrid fiber (i.e. mix of steel cord and polyethylene: $\mathrm{SC}+\mathrm{PE}$ ). The results were also compared with ordinary mortar. While beam specimens were subjected to accelerated corrosion for one year by applying electrical potential of 3 volts across an internal anode (the steel bar) and an external cathode built with wire mesh and placed near the bottom face of the beams, the results showed that the hybrid FRCC exhibited excellent performance compared to mortar and another FRCC containing PE fiber (Figs. $13 \&$ 14). Besides the narrow crack width 
due to bridging of fibers, the self-healing of cracks in these specimens might also contribute to the reduction of steel corrosion. As shown in Homma et al. (2009), cracks in specimens containing PE fibers are closed by the self-healing products and the steel bar embedded in the specimen was more obstructed against the access of chloride ions than that in the mortar specimen.

\subsection{Engineering with admixtures}

In order to stimulate the chemical reaction to produce hydration products for filling cracks in concrete, some admixtures can be used. For example, the application of mineral-producing bacteria and geo-materials has been proposed.

Recently, the precipitation of calcium carbonate due to biochemical action of bacteria has been noticed. According to Jonkers (2010), the principal mechanism of bacterial crack healing is that the bacteria themselves act largely as a catalyst and transform a precursor compound into a suitable filler material. Jonkers pointed out the following two requirements for applying this new technique to a self-healing concrete: (1) The lifetime of the bacteria needs to be long enough, that is equivalent to that of concrete structures; (2) The addition of bacteria or additionally necessary bio-cement precursor compounds should not cause the loss of other properties of the concrete itself. While alkali-resistant spore-forming bacteria existing in nature are viable over 50 years, the lifetime of bacteria in concrete is limited to only a few months when the bacteria spores are added directly to the concrete mixture. Furthermore, a drastic decrease of compressive strength was found because of various organic bio-cement precursor compounds, though a large number of bacteria spores hardly affected the strength.

A technique based on the application of mineral-producing bacteria has been developed in several laboratories. Ramachandran et al. (2001) published a paper describing an innovative biotechnology utilizing microbiologically induced mineral precipitation for concrete remediation. Cracks filled with bacteria and sand demonstrated a significant increase in compressive strength and stiffness when compared with crack without cells.

Van Tittelboom et al. (2010) investigated the use of a biological repair technique in which ureolytic bacteria are able to precipitate $\mathrm{CaCO}_{3}$ in their micro-environment by conversion of urea into ammonium and carbonate. It was shown that cracks were filled completely by protecting bacteria in silica gel against the high $\mathrm{pH}$ in concrete, though pure bacteria cultures were not able to bridge the cracks.

In order to substantially increase the lifetime and associated functionality of concrete incorporated bacteria, Jonkers $(2010,2011)$ immobilized both bacteria spores and a simultaneously needed organic bio-mineral precursor compound (calcium lactate) by applying a vacuum technique in porous expanded clay particles prior to addition to the concrete mixture. The particle size was 1

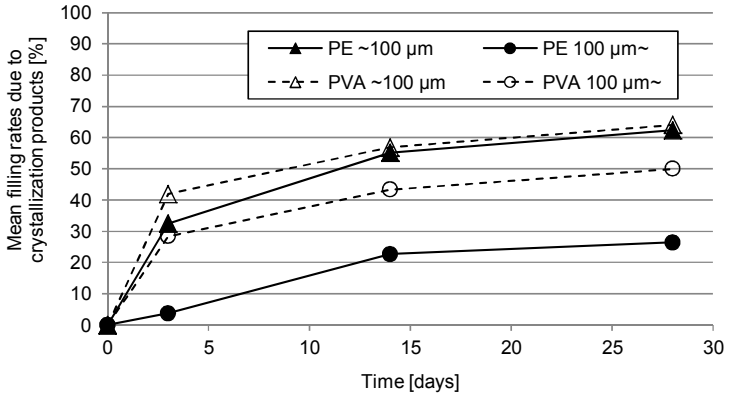

Fig. 12 Relationship between the mean filling rate of crack and the time (Koda et al. 2011).

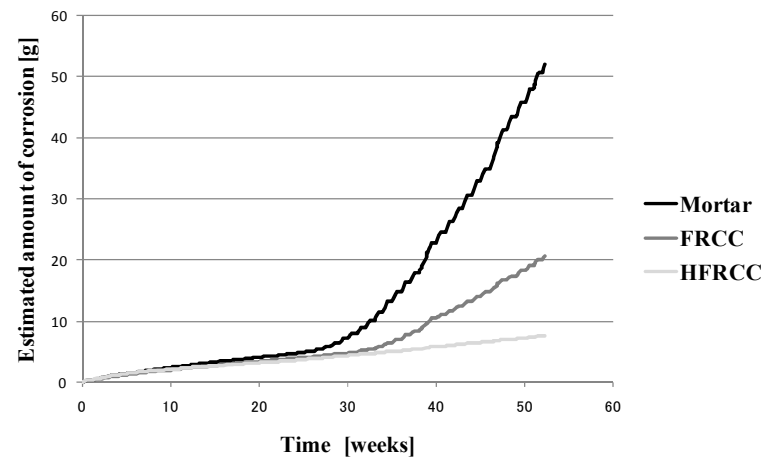

Fig. 13 Time-dependent change of the amount of estimated corrosion (Mihashi et al. 2011).
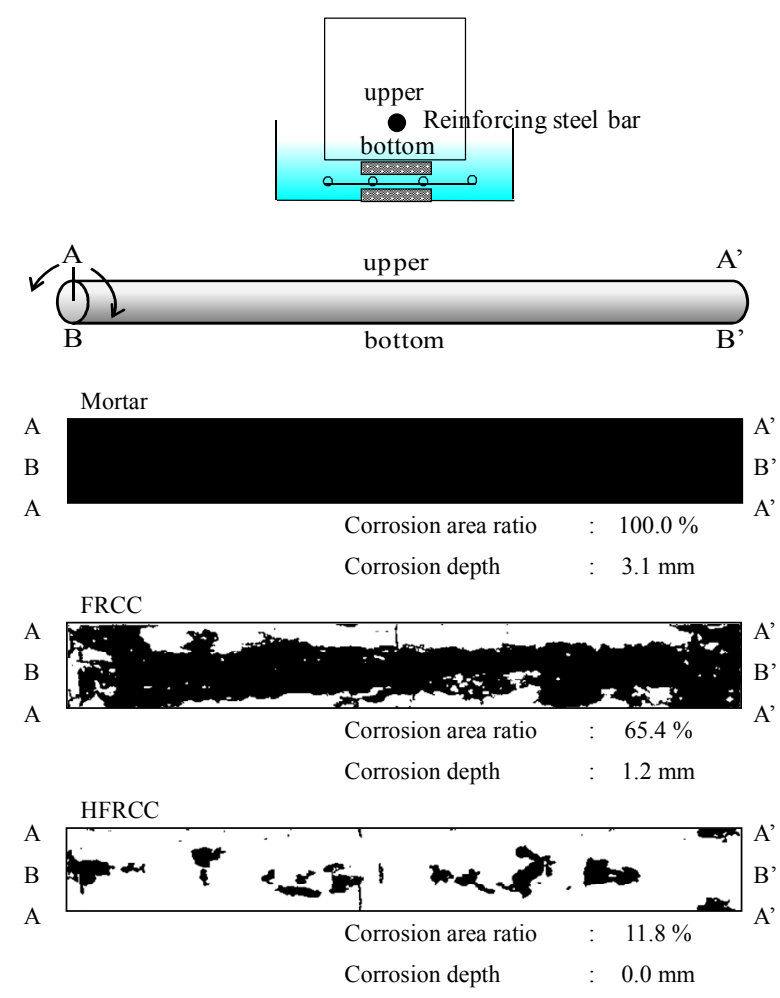

Fig. 14 Development charts of the reinforcing steel bar (Mihashi et al. 2011). 
to $4 \mathrm{~mm}$ and the shape was spherical. Environmental SEM revealed that efficient healing of cracks occurred in the bacteria specimen in which large (50 to $100 \mathrm{mi}-$ crometers) calcium-carbonate-based self-healing products were created. Tests also showed that bacterial spore viability increased from two to more than six months, though this duration is still much shorter than the usual lifetime of buildings and infrastructures.

Ahn and Kishi (2010) developed a self-healing concrete using geo-materials which was available for practical application. They concluded that self-healing capability was significantly affected by aluminosilicate materials and various modified calcium composite materials mainly due to the swelling effect, expansion effect and recrystallization. This approach requires the supply of water or at least moisture, but since most infrastructures are exposed to rain or underground water, usually this is an easily satisfiable requirement.

Taniguchi et al. (2011) studied self-healing of frost-damaged concrete in which sand was replaced with fly ash of $15 \%$ of cement by weight. The water-Portland cement ratio was 0.5 . After undergoing accelerated freeze-thaw cycles until their relative dynamic modulus of elasticity was reduced to $80 \%$, specimens were cured in water at $40^{\circ} \mathrm{C}$ for 28 days as a second curing. In addition to measuring the change in the relative dynamic modulus, carbonation tests were carried out. As a result, reduction of the carbonation rate after the second curing was found to be significant in the case of concrete containing fly ash and entrained air, although the recovery of the relative dynamic modulus of elasticity was comparatively not so good.

Sisomphon et al. (2011) proposed a self-healing system for carbonated blast furnace slag mixtures subjected to carbonation and frost salt scaling attack. They used expanded clay lightweight aggregates (LWA) impregnated with a sodium-monofluorophosphate (referred to as Na-MFP) solution and subsequently coated with a cement paste layer as a healing medium. Na-MFP is one of the corrosion inhibition agents for reinforced concrete. The experimental results revealed that the mortar specimens containing encapsulated LWA had better resistance against frost salt attack and that the capillary water absorption was obviously decreased in comparison with mortar with normal fine aggregates. The conclusion was that the healing mechanism could be due to the reaction of Na-MFP compound and portlandite crystals supplied from the coating layer of Portland cement paste.

\section{Self-repairing}

\subsection{Intelligent materials}

Self-repairing concrete is involved in a category of intelligent materials which is popular definition in various material research communities other than concrete. Intelligent materials possess the following three functions (Fig. 15) (Science and Technology Agency 1989, Mihashi et al. 2000, Mihashi et al. 2002).
(1) Sensing function - for locating or detecting the presence of targeted changes such as cracks.

(2) Processing function - for judging which action should be taken and/or when it should be taken.

(3) Actuating function - for putting the planned repair operations into action.

That is to say, an intelligent material can treat stimuli from the changing external environment as information to process the condition of the material itself. Such functions can be incorporated into cementitious materials not necessarily in the form of the natural healing process and/or ordinary admixtures as mentioned above, but also through the supply of specialized devices to repair cracks. The choice of such devices includes sensing devices, tubular network-like vessels, and synthetic resins that harden without the presence of water. Functions are given by different substances from the original constitutions of concrete. This concept was defined as "Activated Repairing" by the JCI Technical Committee on Autogeneous-Healing in Cementitious Materials as shown above (Fig. 1(a)).

\subsection{Passive self-repairing}

The Science and Technology Agency (1989) suggested the basic concept of intelligent materials composed of distributed functional capsules and matrices (Fig. 15). This concept was widely recognized as a practical research topic for research on polymeric materials using encapsulated chemicals (White et al. 2001). Figure 16 shows a conceptual diagram of self-healing. In the field of concrete engineering, there were some researchers who adopted a similar concept, i.e. mixing brittle capsules containing healing agent in concrete. One of the earliest studies in the field of concrete engineering was carried out by Dry (1994). In this study, an adhesive agent contained in hollow brittle glass fibers served as the repairing chemicals. A crack in a brittle cementitious

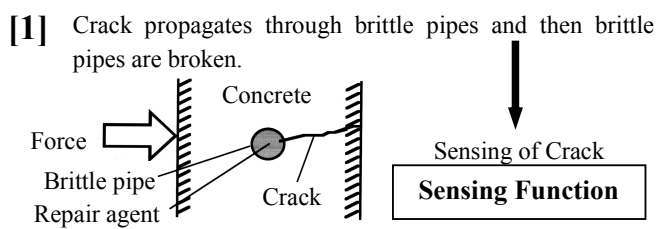

[2] Repair agent is released from the broken pipe.

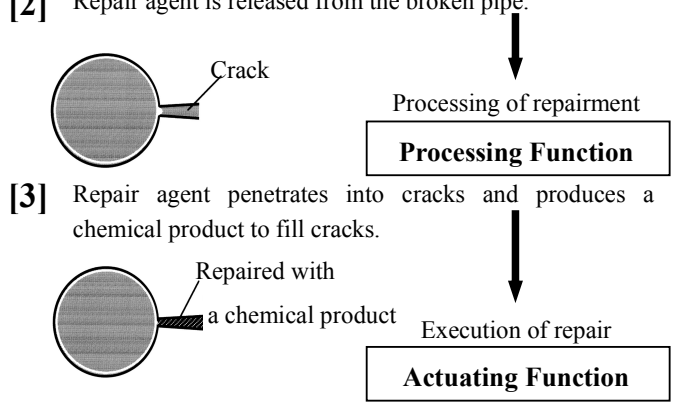

Smart Material with Self-Repairing Function

Fig. 15 Schematic description of the self-healing system (Science and Technology Agency 1989, Mihashi et al. 2000, Mihashi et al. 2002). 


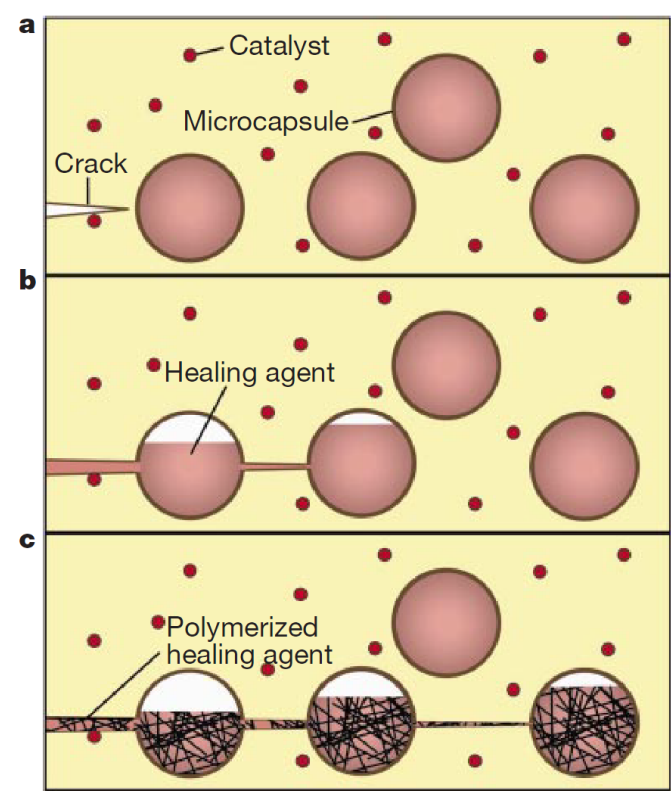

Fig. 16 The autonomic healing concept (White et al. 2001).

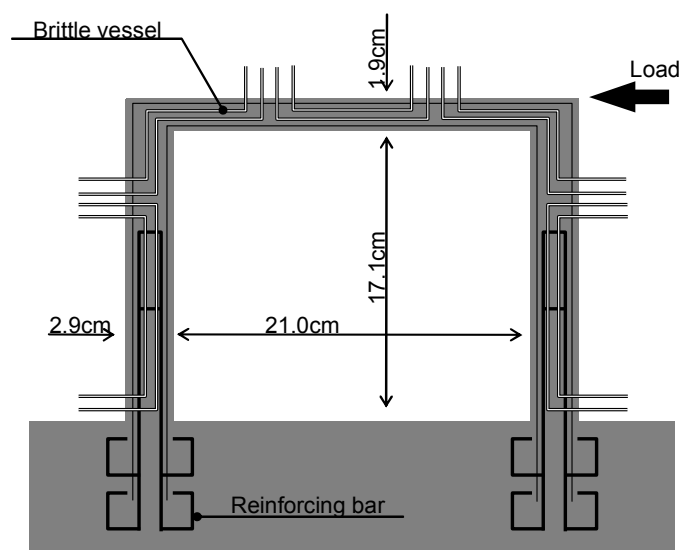

Fig. 17 Sample test frame (Dry 2001).

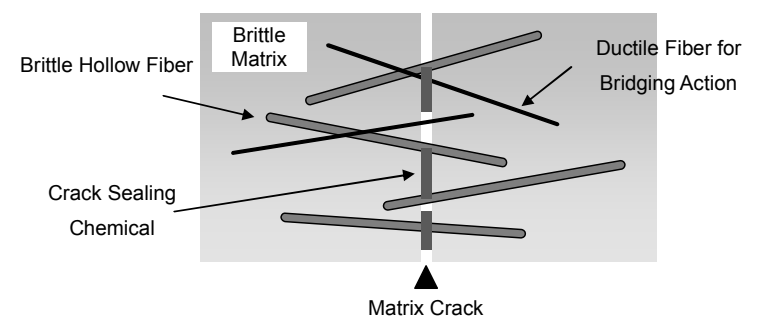

Fig. 18 PSS-ECC proposed by Li (drawn based on Li 1995).

material caused by overloading was detected by breaking the fibers. The resulting tensile cracking of the matrix and breaking of the glass fibers stimulate the actuating mechanisms to repair the crack in this totally passive smart material system. The adhesive agent can be released into and fill up the crack. Thereafter, Dry investigated some applications of this idea to concrete structures (Dry 1998 and Dry 2001). Figure 17 shows the employed specimen, a plane, one-story, rigid portal frame cast monolithically with a concrete base. The chemically inert tubing was cast within the cross section of the member and then filled with adhesive agents. As adhesive agents with different characteristics could be applied, a series of experimental investigations was carried out to evaluate the ability of three different adhesive agents, i.e. cyanoacrylate, two-part epoxy resin, and silicon based adhesive agent. In order to evaluate the efficiency of each adhesive agent for self-repairing, changes in the stiffness and frequency of cracking were monitored during loading. Both of these parameters showed that cyanoacrylate adhesive agent was the most effective for recovering stiffness and preventing crack reopening.

Li et al. (1998) studied a Passive Smart Self-healing Engineered Cementitious Composite (PSS-ECC). The PSS-ECC in that study consisted of brittle glass tubes containing superglue (ethyl cyanoacrylate) embedded in ECC (Fig. 18). The authors emphasized that controlling the crack width within tens of micrometers is essential for PSS-ECC because wider crack widths rapidly exhaust the amount of adhesive agent available for healing the cracks. Otherwise, very thick hollow glass tubes are necessary, which in turn reduces the mechanical properties of the FRCC. Moreover, the maximum allowable crack width should be also limited by the actuation mechanism because the release of adhesive agents into the crack is dependent on the capillarity of the thin channels created by the crack surfaces against the capillary force inside the hollow glass fibers. Thus, the crack width of the matrix should be limited to less than the inner diameter of the glass fiber for effective actuation.

Nishiwaki et al. (2004) investigated a passive self-repairing system composed of embedded brittle glass tubes and an HPFRCC matrix. The repair agent was selected to recover not mechanical properties but water tightness, and a water permeability test was carried out on the pre- and post-cracked specimens. Figure 19 shows the relation between the maximum crack width (wR) and the calculated coefficient of water permeability (K). The results showed that the self-healing system was effective in FRCC specimens with maximum crack widths larger than $0.2 \mathrm{~mm}$, but also that it was often ineffective when the maximum crack width was below this value because of the viscosity of the repair agent.

\subsection{Active self-repairing}

Since cementitious materials including concrete are brittle, all passive repairing systems such as those mentioned above require brittle containers for preserving self-repairing agents, and these container need to break as soon as critical cracks occur in order to release the agents. However, handling of such fragile devices is quite difficult at construction sites. Allowing the introduction of minimum inputs such as electrification or heating could greatly increase the selection of devices that can be used. 


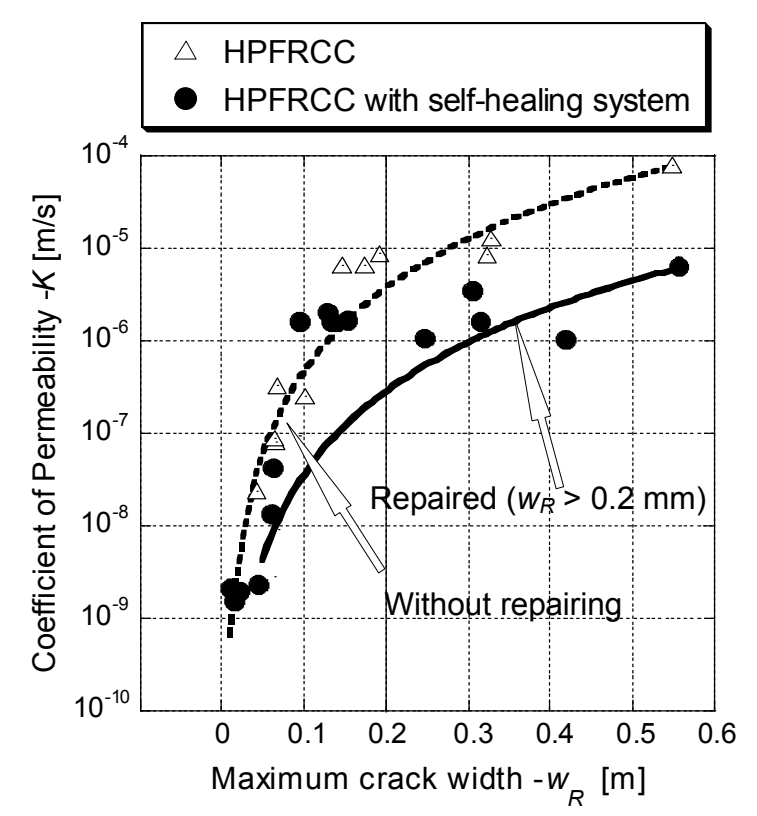

Fig. 19 Relationship $K-w_{R}$ (Nishiwaki et al. 2004).

A crack-closure system using Shape Memory Alloy (SMA) was proposed by Sakai et al. (2003). They used SMA as main reinforcing bars for concrete beams in order for large cracks under loading to be mechanically closed after unloading, although total crack closure was elusive.

Jefferson et al. (2010) developed a similar crack-closure system for cementitious materials using shrinkable polymer tendons. The system involved the incorporation of unbonded pre-oriented polymer tendons in cementitious beams. Cracks can be closed through thermal activation of the shrinkage mechanism of the restrained polymer tendons (Fig. 20). However, if external human intervention is required for heating the structure to close cracks, the material does not qualify as an intelligent material as defined above. Moreover, too much energy would be required to activate the system if the whole structure needs to be heated.

Nishiwaki et al. (2006a) developed a new approach to achieve a self-repairing function in a totally different way from the previous technologies including passive ones. That approach could be called an "active self-repairing system." This system automatically starts in response to electrical signals triggered by cracking in concrete. The system consists of a conductive composite for self-diagnosis of cracking and pipes made with heat-plasticity film that contains a low viscosity epoxy resin as a repairing agent. The self-diagnosis composite is a kind of crack monitoring sensor and at the same time it has the function of a heating device for a specific location through electrification. The self-diagnosis composite is fabricated using fiber-reinforced composites and conductive particles (Fig. 21). In the absence of any damage, the sensor can monitor the strain due to the electrical conduction path with dispersed conductive particles. When the sensor detects a localized large strain due to the formation of a crack in the concrete, its electrical resistance increases since part of the electrical conduction path is cut off around the crack. The sensor

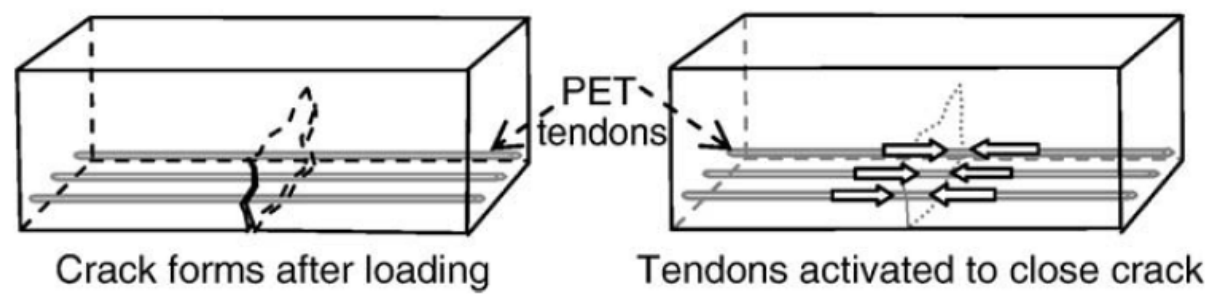

Fig. 20 Schematic illustration of concept for new composite material system (Jefferson et al. 2010).

Cutting of conductive path by partial strain

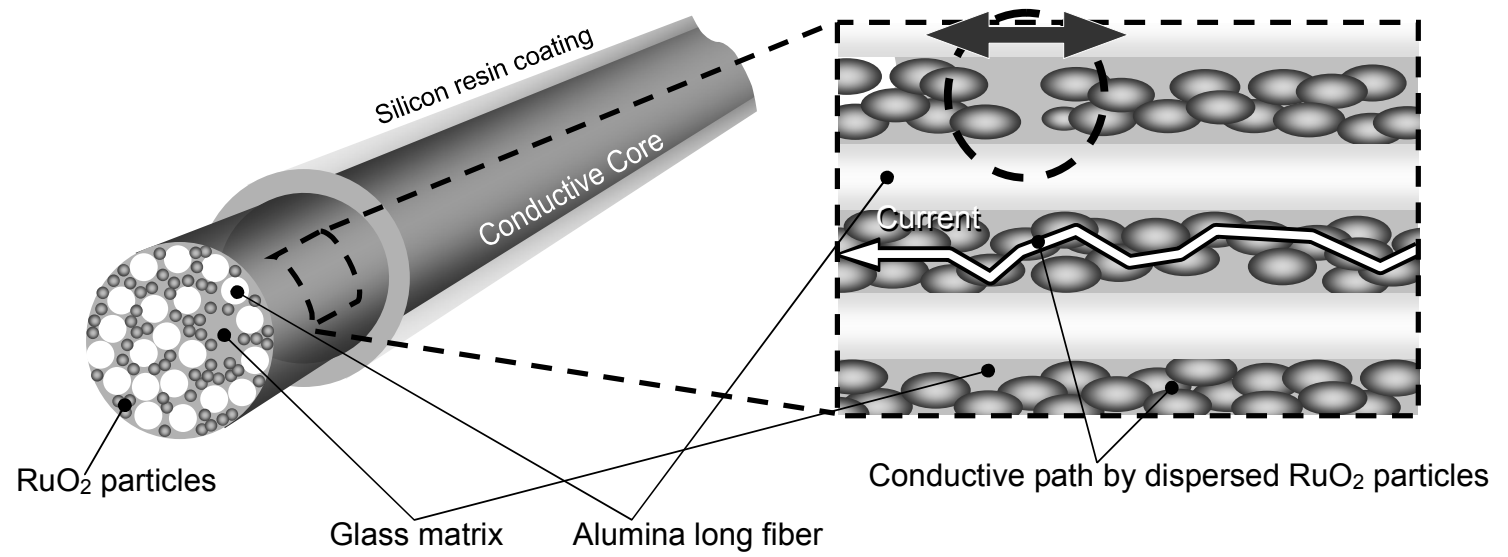

Fig. 21 Schematic diagram of self-diagnosis composite structure (Nishiwaki et al. 2006a). 


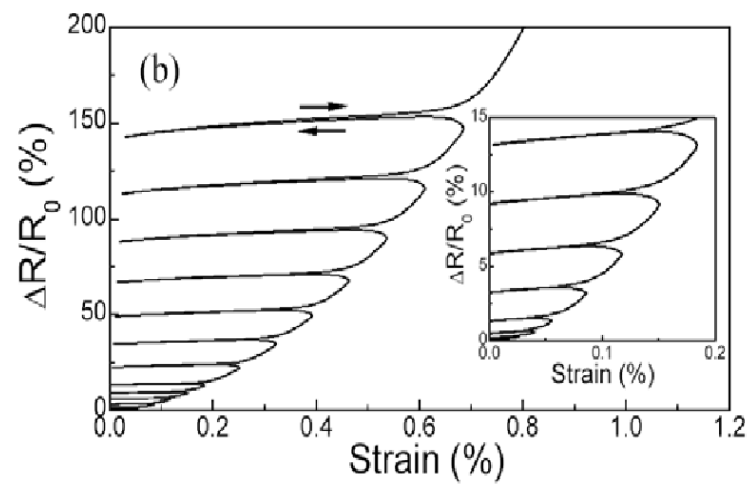

Fig.22 Strain vs. resistance change ratio relationship of strain monitoring sensor under tensile cyclic load (Mihashi et al. 2008).

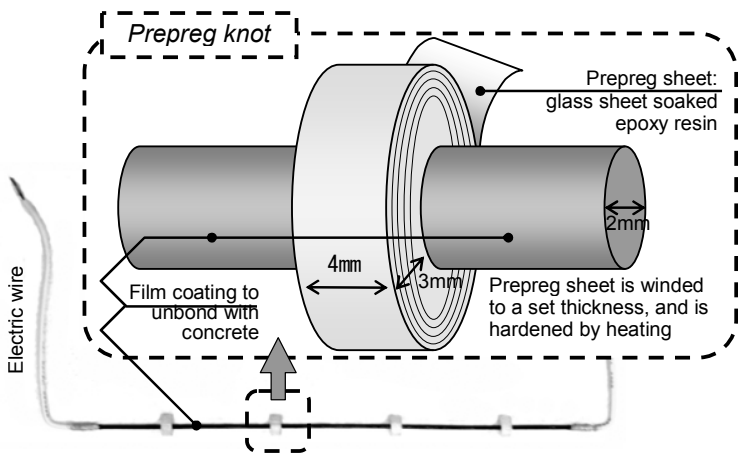

Fig. 23 Schematic diagram of prepreg knot and the self-diagnosis composite. (Nishiwaki et al. 2007).

can then delicately increase the resistance even in the case of a very small strain due to the dispersive structure of the conductive particles (Fig. 22). By means of electrification in this sensor, a partial increase in electrical resistance can achieve selective heating around the crack. In these sensors, different conductive particles such as $\mathrm{RuO}_{2}$ and carbon black particles can be used for different monitoring targets. Moreover, different types of conductive particles yield various calorific values under the same electrification condition because of the different resistance ratios of the sensors (Nishiwaki et al. 2006b).

In order to investigate the quantitative relation be-

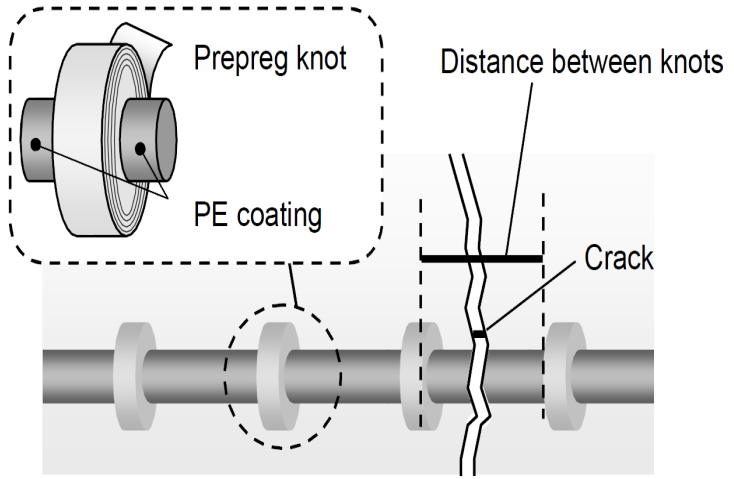

Fig. 24 Schematic diagram of crack monitoring sensor (Mihashi et al. 2008).

tween the strain of the diagnosis and the crack width, Nishiwaki et al. (2007) installed nodes on the surface of the self-diagnosis composite as shown in Fig. 23. Between these nodes, the self-diagnosis composite was coated with polyethylene film to eliminate the bond between the concrete and the self-diagnosis composite (Fig. 24). Thus the crack width was directly related to the measured strain with some accuracy.

A schematic diagram of this self-repairing system is shown in Fig. 25 (Nishiwaki et al. 2010). In this system, copper plates were employed to connect the heating device and the melting pipe for smooth thermal transfer. Once selective heating at the location of the crack starts, the plastic pipe at the crack is melted to supply the epoxy resin to the crack. The heat-plasticity pipe seals off the repair agent inside the concrete in order to prevent its hardening reaction and the melting point of the film should be low enough to allow easy melting of the film by heating around the crack. For that purpose, an ethylene vinyl acetate polymer film with a melting point of $93^{\circ} \mathrm{C}$ was employed.

Figure 26 (Mihashi et al. 2008) shows a schematic description of a specimen and the test setup that was used for confirming the function of the self-diagnosis composite. The effectiveness of the heating function was proved by means of direct observation with an infrared

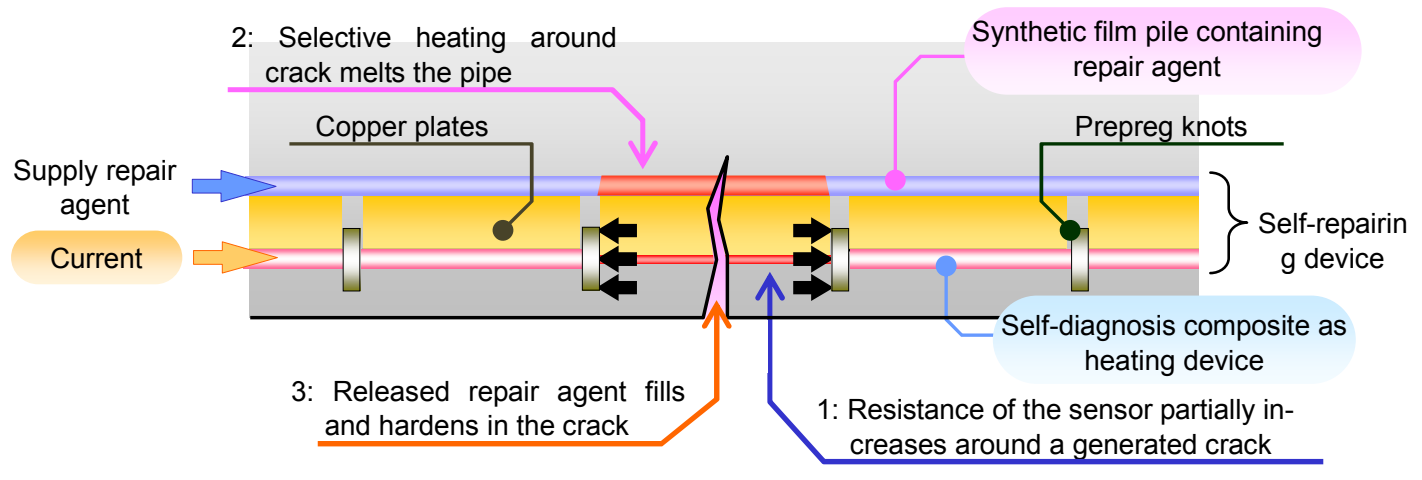

Fig. 25 Schematic illustration of activated repairing system (Nishiwaki et al. 2010). 


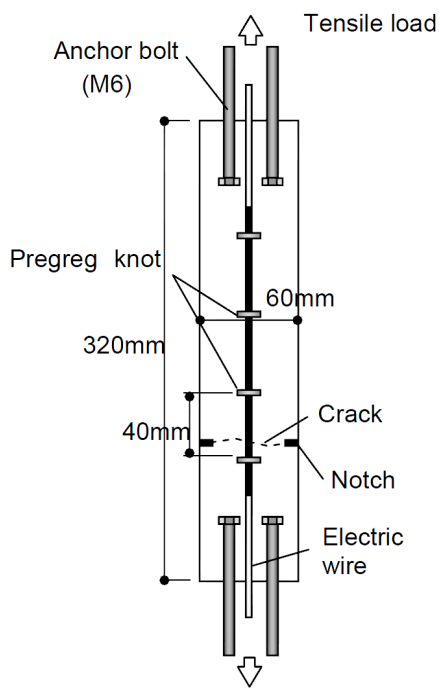

(a) Tested specimen

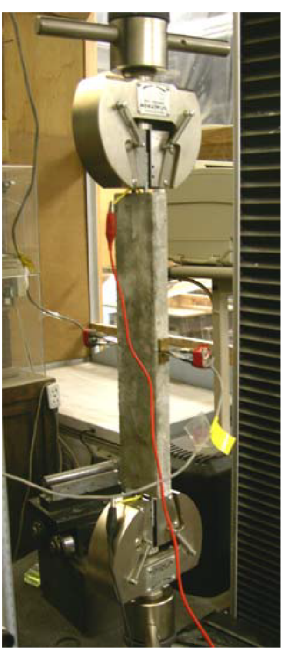

(b) Test set-up for tensile loading

Fig. 26 Schematic description of the specimen and the test setup (Mihashi et al. 2008).

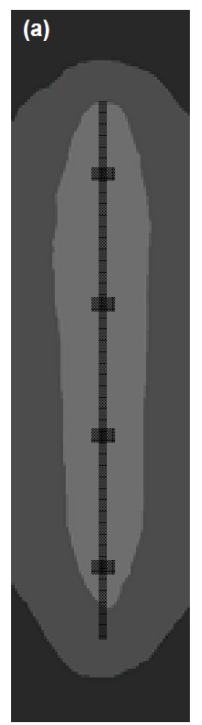

(a) Before cracking

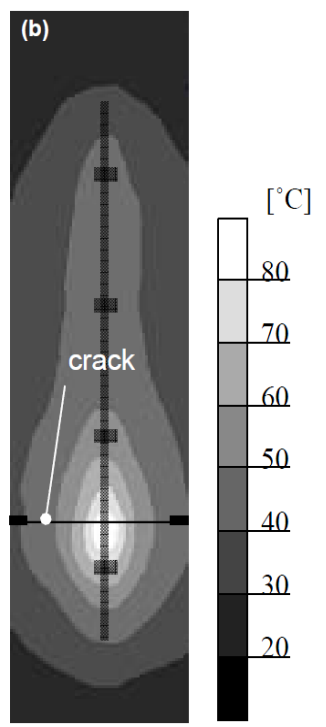

(b) After cracking

Fig. 27 Temperature distribution measured by thermography (Mihashi et al. 2008).

radiation thermography technique as shown in Fig. 27. Figure 28 shows an example of the experimental results of the permeability test to prove the effectiveness of the self-repairing system (Nishiwaki et al. 2010). Figure 29 shows an example of load-displacement curves of specimens with and without the self-repairing system. The whole results are shown in Fig. 30, in which the recovery of strength and stiffness by the system is obvious (Nishiwaki et al. 2009).

\section{Concluding remarks}

This paper has reviewed previous studies on self-healing and self-repairing of concrete. The following concluding remarks can be made.

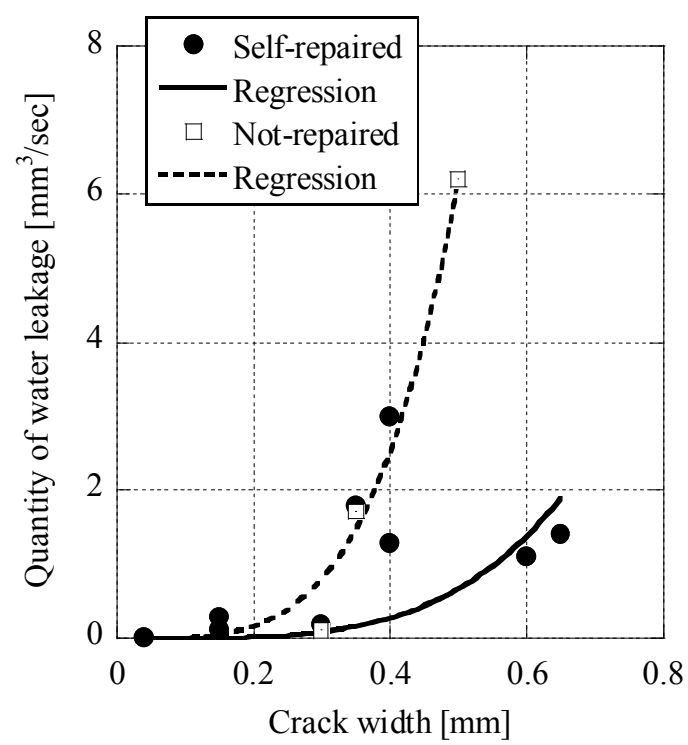

Fig. 28 Relationship between the generated maximum crack width versus the quantity of water leakage (Nishiwaki et al. 2010).

(1) Fiber reinforced cementitious composites (FRCC) have higher self-healing potential than ordinary plain concrete. Fibers bridging the crack can reduce the crack width and furthermore they can work as cores for the precipitation of calcium carbonate in the crack. However, the latter mechanism can function only when water or at least moisture is supplied.

(2) Precipitation of calcium carbonate due to biochemical action of bacteria has been expected to be one of the new technologies for self-healing of concrete, though there are still problems, such as the fact that the lifetime of bacteria in concrete is much shorter than the service life of buildings and infrastructures. 


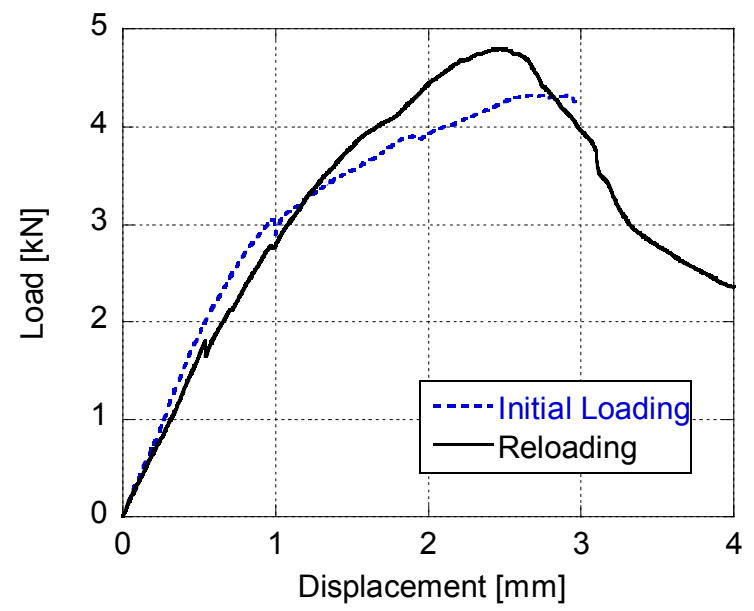

(a) Self-repaired

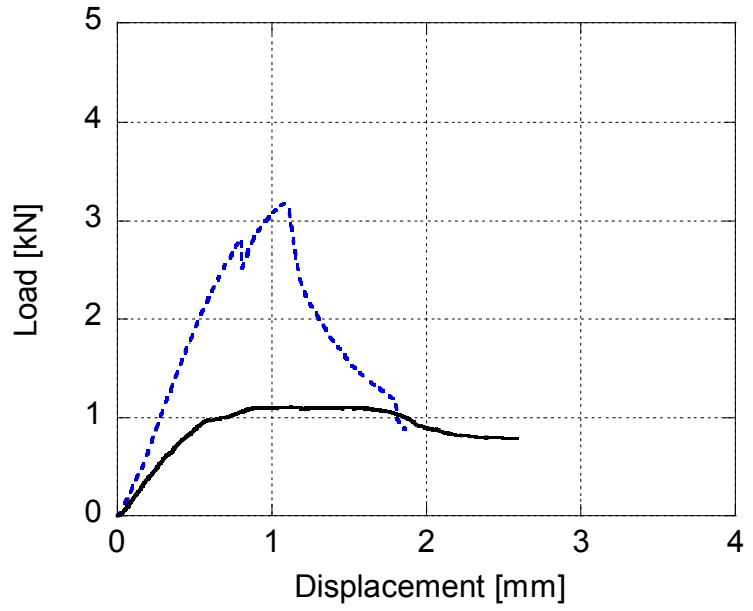

(b) Without repair

Fig. 29 Examples of load-displacement curve (Nishiwaki et al. 2009).

(3) At present, the geomaterial approach using mineral admixtures seems to be more practical in the field of civil engineering than other self-healing technologies. While this approach requires the supply of water or at least moisture, most infrastructures are exposed to rain or underground water.

(4) Self-repairing concrete has the potential to be an intelligent material and it can be applied to specific concrete structures for which ordinary means of repairing cracks are not available owing to engineer safety considerations or inaccessible location.

In closing, engineered self-healing and self-repairing concrete hold promise for longer lasting concrete structures and we expect significant development of this field in the future.

\section{References}

Ahn, T.-H. and Kishi, T., (2010). "Crack self-healing behavior of cementitious composites incorporating various mineral admixtures." Journal of Advanced Concrete Technology, 8(2), 171-186.

Aldea, C.-M., Song, W.-J., Popovics, J. S. and Shah, S. P.,

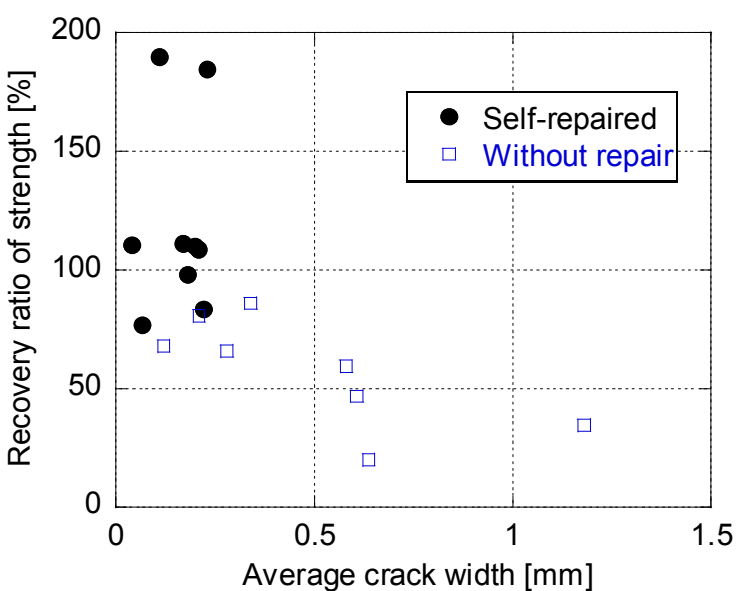

(a) Strength

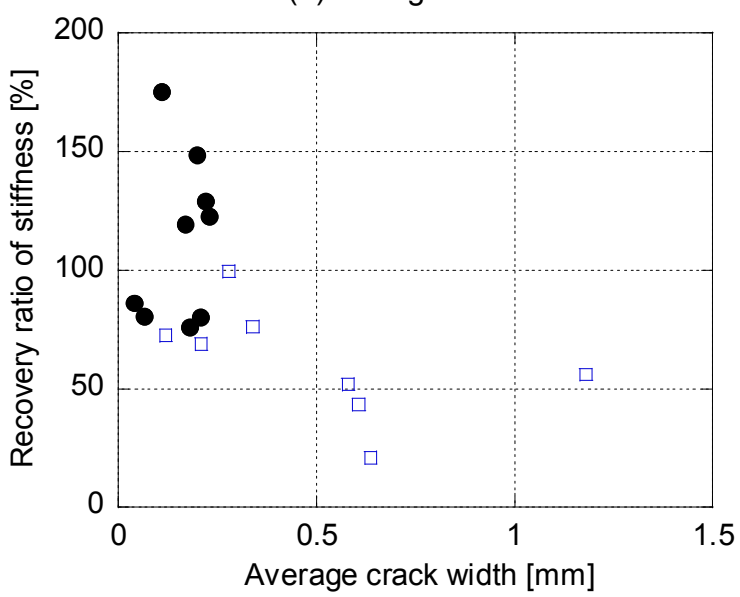

(b) Stiffness

Fig. 30 Relationship between average crack width and recovery ratio of strength and stiffness (Nishiwaki et al. 2009).

(2000). "Extent of healing of cracked normal strength concrete." Journal of Materials in Civil Engineering, 12(1), 92-96.

De Rooij, M. R. and E. Schlangen (eds.) (2011). "Self-healing phenomena in cement-based materials." Draft of State-of-the-Art Report of RILEM Technical Committee 221-SHC.

Dhir, R. K., Sangha, C. M. and Munday, J. G. L., (1973). "Strength and deformation properties of autogenously healed mortars." Journal of the American Concrete Institute, 70(3), 231-236.

Dry, C.M., (1994). "Smart multiphase composite materials that repair themselves by a release of liquids that become solids." Proceedings of SPIE, 2189, 62-70.

Dry, C. M., (1998). "Preserving Performance of Concrete Members under Seismic Loading Conditions." Proceedings of SPIE, 3325, 74-80.

Dry, C. M., (2000). "Three designs for the internal release of sealants, adhesives, and waterproofing chemicals into concrete to reduce permeability." Cement and Concrete Research, 30(12), 1969-1977. 
Dry, C. M., (2001). "Design of Self-growing, Self-sensing and Self-repairing Materials for Engineering Applications." Proceedings of SPIE, 4234, 23-29.

Edvardsen, C., (1999). "Water permeability and autogenous healing of cracks in concrete." $A C I$ Material Journal, 96(4), 448-454.

Grey, D. J., (1984). “Autogenous healing of fiber/matrix interfacial bond in fiber-reinforced mortar." Cement and Concrete Research, 14, 315-317.

Granger, S., Loukili, A., Pijaudier-Cabot, G. and Chanvillard, G., (2007). "Experimental characterization of the self-healing of cracks in an ultra high performance cementitious material: mechanical tests and acoustic emission analysis." Cement and Concrete Research, 37(4), 519-527.

Hannant, D. J. and Keer, J. G., (1983). "Autogenous healing of thin cement based sheets." Cement and Concrete Research, 13(3), 357-365.

Hearn, N. and Morley, C. T., (1997). "Self-healing property of concrete - experimental evidence." Materials and Structures, 30(201), 404-411.

Hearn, N.(1998). "Self-sealing, autogenous healing and continued hydration: what is the difference?" Materials and Structures, 31(212), 563-567.

Herbert, E. N. and Li, V. C., (2011). "Self-healing of engineered cementitious composites in the natural environment." High Performance Fiber Reinforced Cement Composites 6, G. J. Parra-Montesinos, H. W. Reinhardt and A. E. Naaman (eds.), Springer, 155-162.

Homma, D., Mihashi, H. and Nishiwaki, T., (2009). "Self-healing capability of fiber reinforced cementitious composites." Journal of Advanced Concrete Technology, 7(2), 217-228.

Homma, D., Mihashi, H. and Nishiwaki, T., (2008). "Experimental study of the self-healing capability of fiber reinforced cementitious composites." in Fiber Reinforced Concrete: Design and Applications, R. Gettu (ed.), RILEM Publications, S.A.L., 1029-1038.

Igarashi, S., Kunieda, M. and Nishiwaki, T., (2009). "Research activity of JCI technical committee TC-075B: Autogenous healing in cementitious materials." Proceedings of 4th International Conference on Construction Materials: Performance, Innovations and Structural Implications, ConMat'09 (Nagoya, Japan), JCI, Keynote Lecture, 89-96.

Jacobsen, S., Marchand, J. and Hornain, H., (1995). "SEM observation of the microstructure of frost deteriorated and self-healed concrete." Cement and Concrete Research, 25(8), 1781-1790.

Jacobsen, S. and Sellevold, E. J., (1996). "Self healing of high strength concrete after deterioration by freeze/thaw." Cement and Concrete Research, 26(1), 55-62.

Jacobsen, S., Marchand, J. and Gerard, B., (1998). "Concrete cracks I: durability and self-healing - a review." In Concrete under Severe Conditions 2, Gjorv,
O.E., Sakai, K. and Banthia, N. (eds.), London; New York, E \& FN Spon, 217-231.

Japan Concrete Institute (2009). "Autogenous-healing in cementitious materials." Technical Committee Report of JCI-TC075 B, 179p. (in Japanese)

Jefferson, A., Joseph, C., Lark, R., Isaacs, B., Dunn, S. and Weager, B., (2010). "A new system for crack closure of cementitious materials using shrinkable polymers." Cement and Concrete Research, 40, 795-801.

Jonkers, H. M., (2010). "Self-healing of cracks in concrete using a bacterial approach." in Frontiers of Self-healing Materials and Applications, N. Shinya (ed.), CMC Publishers, 194-203.

Jonkers, H. M., (2011). "Bacteria-based self-healing concrete." Heron, 56(1/2), 1-12.

Koda, M., Mihashi, H., Nishiwaki, T., Kikuta, T. and Kwon, S.-M., (2011). "Self-healing capability of fiber reinforced cementitious composites." Proc. of Int'l Workshop on Advances in Construction Materials through Science and Technology, Hong Kong, RILEM PRO 79.

Lauer, K. R. and Slate, F. O., (1956). "Autogenous healing of cement paste." Journal of the American Concrete Institute, 52(6), 1083-1097.

Li, V. C., (1995). "Development of passive smart self-healing cementitious composites at Univ. Michigan." Private communication.

Li, V. C., Lim, Y. M. and Chan, Y-W., (1998). "Feasibility study of a passive smart self-healing cementitious composite" Composites Part B, 29B, 819-827.

McHenry, D. and Brewer, H. W., (1945). "Discussion of a paper by T.C. Powers: A working hypothesis for further studies of frost resistance of concrete." Journal of American Concrete Institute, 41(supplement), 272.9-12.

Mihashi, H., Kaneko, Y., Nishiwaki, T. and Otsuka, K., (2000). "Fundamental study on development of intelligent concrete characterized by self-healing capability for strength." Transactions of the Japan Concrete Institute, 22, 441-450.

Mihashi, H., Nishiwaki, T., Kaneko, Y. and Nishiyama, N., (2002) "Development of smart concrete." Concrete Structures in the $21^{\text {st }}$ Century, Proceedings of the $1^{\text {st }}$ fib Congress, 6, 165-174.

Mihashi, H., Nishiwaki, T., Miura, K. and Okuhara Y., (2008). "Advanced Monitoring Sensor and Self-Repairing System for Cracks in Concrete Structures." Proceedings of SACoMaTiS 2008 (Como Lake, Italy), RILEM, 1, 401-409.

Mihashi, H., Ahmed, S. F. U. and Kobayakawa, A., (2011). "Corrosion of reinforcing steel in fiber reinforced cementitious composites." Journal of Advanced Concrete Technology, 9(2), 159-167.

Nishiwaki, T., Leite, J. P. de B. and Mihashi, H., (2004). "Enhancement in Durability of Concrete Structures with Use of High-Performance Fibre Reinforced 
Cementitious Composites." Proceedings of the $4^{\text {th }}$ International Conference on Concrete under Severe Conditions: Environment \& Loading; CONSEC'04, 2, 1524-1531.

Nishiwaki, T., Mihashi, H., Jang, B. K. and Miura, K., (2006a). "Development of self-healing system for concrete with selective heating around crack." Journal of Advanced Concrete Technology, 4(2), 267-275.

Nishiwaki, T., Mihashi, H., Miura, K., Okuhara, Y. and Matsubara, H., (2006b). "Smart concrete system with strain monitoring sensors including conductive particles." Proceedings of the $2^{\text {nd }}$ International Symposium on Advances in Concrete through Science and Engineering, 11-13 September 2006, Quebec City, Canada, RILEM Pro051, Marchand, J., Bissonnette, B., Gagne, R., Jolin, M. and Parac, F. (eds.), CD-ROM.

Nishiwaki, T., Mihashi, H. Gunji, Y. and Okuhara, Y., (2007). "Development of smart concrete with self-healing system using selective heating device." Proceedings of the $5^{\text {th }}$ International Conference on Concrete under Severe Conditions: Environment \& Loading; CONSEC'07, Tours, France, Toutlemonde F. et al. (eds), 1, 665-672.

Nishiwaki, T., Mihashi, H. and Okuhara, Y., (2009). "Experimental study on a recovery assessment of effectiveness of a self-repairing concrete." Proceedings of Japan Concrete Institute, 31(1), 2167-2172. (in Japanese)

Nishiwaki, T., Mihashi, H. and Okuhara, Y., (2010). "Fundamental study on self-repairing concrete using a selective heating device." Proceedings of the $5^{\text {th }}$ International Conference on Concrete under Severe Conditions: Environment \& Loading; CONSEC'10 (Mérida, Yucatán, México), 2, 665-926.

Ramachandran, S. K., Ramakrishnan, V. and Bang, S. S., (2001). "Remediation of concrete using micro-organisms." ACI Material Journal, 98(1), 3-9.

Ramm, W. and Biscoping, M., (1998). "Autogeneous healing and reinforcement corrosion of water-penetrated separation cracks in reinforced concrete." Nuclear Engineering and Design, 179(2), 191-200.

Reinhardt, H.-W. and Jooss, M., (2003). "Permeability and self-healing of cracked concrete as a function of temperature and crack width." Cement and Concrete
Research, 33(7), 981-985.

Sakai, Y., Kitagawa, Y., Fukuta, T. and Iiba, M., (2003). "Experimental study on enhancement of self-restoration of concrete beams using SMA wire." Proceedings of SPIE, 5057, 178-186.

Sanjuan, M. A., Andrade, C. and Bentur, A., (1997). "Effect of crack control in mortars containing polypropylene fibers on the corrosion of steel in a cementitious matrix." ACI Materials Journal, 94, 134-141.

Science and Technology Agency (1989). "Report for overtures No. 13 in the council of aeronautical and electronic technology." 1-82. (in Japanese)

Sisomphon, K., Copuroglu, O. and Fraaij, A., (2011). "Application of encapsulated lightweight aggregate impregnated with sodium monofluorophosphate as a self-healing agent in blast furnace slag mortar." Heron, 56(1/2), 13-32.

Shahinpoor, M., (1997). "Intelligent civil engineering materials, structures and systems revisited." in Intelligent Civil Engineering Materials and Structures, F. Ansari, A. Maji, and C. Leung (eds.), New York, ASCE, 44-61.

Taniguchi, M., Hama, Y., Katsura, O., Sagawa, T. and Na, S. H., (2011). "Self healing of frost-damaged concrete incorporating fly ash." Proc. $3^{\text {rd }}$ International Conference on Self-Healing Materials, Bath, U.K. 27-29 June.

Van Tittelboom, K., de Belie, N., de Muynck, W. and Verstraete, W., (2010). "Use of bacteria to repair cracks in concrete." Cement and Concrete Research, 40(1), 157-166.

White, S. R., Sottos, N. R., Geubelle, P. H., Moore, J. S., Kessler, M. R., Sriram, S. R., Brown, E. N. and Viswanathan, S., (2001). "Autonomic healing of polymer composites." Nature, 409, 794-797.

Yang, Y.-Z., Lepech, M. D. and Li, V. C., (2005). "Self-healing of engineered cementitious composites under cyclic wetting and drying." in Durability of Reinforced Concrete under Combined Mechanical and Climatic Loads, T.-J. Zhao, F.H. Wittmann and T. Ueda (eds.), Aedificatio Publishers, 231-242.

Yang, Y.-Z., Lepech, M. D., Yang, E.-H. and Li, V. C., (2009). "Autogenous healing of engineered cementitious composites under wet-dry cycles." Cement and Concrete Research, 39(5), 382-390. 


\title{
Self Healing of Concrete Structures - Novel Approach Using Porous Network Concrete
}

\author{
Senot Sangadji ${ }^{1,2}$ and Erik Schlangen ${ }^{1}$
}

\begin{abstract}
To repair concrete cracks in difficult or dangerous conditions such as underground structures or hazardous liquid containers, self healing mechanism is a promising alternative method. This research aims to imitate the bone self healing process by putting porous concrete internally in the concrete structure to create a porous network similar to 'spongious bone'. When cracks are formed and detected by sensors, healing agent can be infused into the porous network so as to fill up voids and seal a crack or cracks in the concrete body. This idea was tested using cylindrical and beam samples. A porous concrete core was placed in the concrete specimens. Uniaxial tensile load in the case of the cylindrical samples and bending load in case of beams was applied to create cracks. A healing action was performed by injecting healing agent manually. The results show that a macro-crack is sealed and strength of concrete is regained. Therefore, the concept is considered as to be feasible for self repair mechanism in concrete.
\end{abstract}

\section{Introduction}

Generally, public has expectations of very long servicelive of infrastructures, not only 50 years as in expected design life, but more like 'last-forever'. However, many constructed infrastructure, e.g. building, concrete structure, transport facilities, built in the second half of the last century is rapidly approaching its critical period marked by reduced functionality due to material deterioration. In contrary, exponential urban population growth has caused increasing public demand of infrastructure that serves their need in constant high level of service.

Van Breugel (2007) presented graphs (see Fig. 1) describing the performance of structures with elapse of time. Gradual degradation occurs until the moment that first repair is urgently needed. Yet there is still a point of concern which is the durability of infrastructures repairs Very often a second repair is necessary only ten to fifteen years later. Spending more money initially in order to ensure a higher quality often pays off. The maintenance-free period will be longer and the first major repair work can often be postponed for many years.

Many scientists and engineers are now looking for the 'right key' for designing structures that show higher durability and have longer 'maintenance free' performance with low repair cost. Society wasted huge amounts of money due to the low quality and durability of concrete and road structures and its ecological impact. In Europe, $50 \%$ of the annual construction budget is esti-

\footnotetext{
${ }^{1}$ Microlab, Faculty of Civil Engineering and Geosciences, Delft University of Technology, the Netherlands.

E-mail: H.E.J.G.Schlangen@tudelft.nl

${ }^{2}$ Civil Engineering Department, Faculty of Engineering, Universitas Sebelas Maret, Solo, Indonesia.
}

mated to be spent on rehabilitation and repair of the existing structures. In US, the average cost of bridge maintenance and repair is $\$ 5.2$ billion. In addition, the costs due to traffic jam are more than 10 times direct maintenance and repair cost. Furthermore, based on DEFRA, up to $50 \%$ of $\mathrm{CO}_{2}$ emission can be associated to building and construction industry.

Van Breugel (2007) argued, "Enhancing the longevity of our built infrastructure will undoubtedly reduce the impact of mankind's activities on the stability of the biosphere". For instance, enhanced infra-structure service life will lessen the demand of new infrastructures resulting low raw material usage. On its turn, it reduces energy consumption and decrease related $\mathrm{CO}_{2}$ emission.

From the material point of view, the effort to increase service life of infrastructure can be made by using various high quality materials, including the new emerging concepts of self healing materials. Nature provides many lesson as biological materials show capabilities to

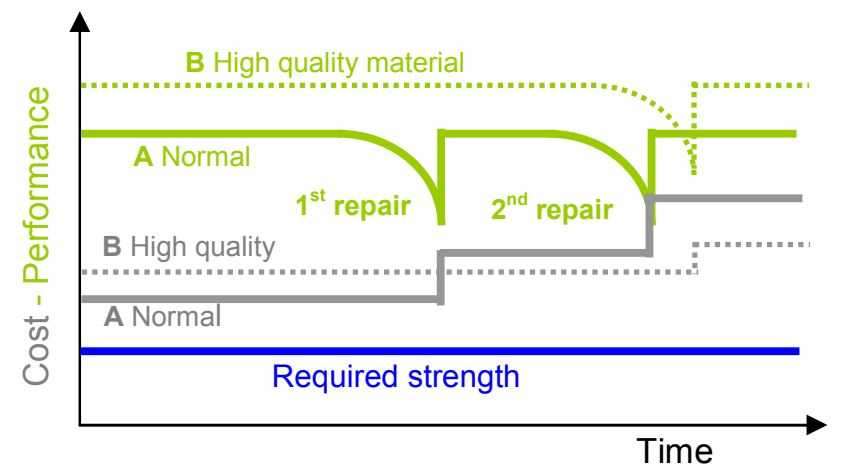

Fig. 1 Performance and cost, including direct repair cost, versus elapse time for $(A)$ normal and $(B)$ high quality infrastructure. External economic parameter neglected (according to Van Breugel 2007). 
heal it self by neutralize wound or injury to reach its previous performance.

Along with the damage management paradigm observed in nature as proposed by Van der Zwaag (2007), many scientists have developed self-healing materials that mimics many of the features of a biological system. Many techniques and methods have been developed according to the intrinsic properties between the various material classes. However, the common feature is all of these self healing materials are able to sense 'damage' and self repair, thus, demonstrate 'continuous renewal' its performance. This results to longer material life time.

For infrastructure the ideal case would be that no costs for maintenance and repair have to be considered at all because the material is able to repair itself as depicted in Fig. 2, (Van Breugel, 2007).

In the past, quite some investigations on the topic of self healing of concrete have been conducted. Neville (2002) gives a useful overview of his literature search in this field. He puts the practical significance of autogenous healing in the reduction of water transport through cracks, for example in concrete water pipes. Neville also concludes from his literature research that there is no agreement between different studies about what happens inside the crack when self healing occurs and therefore further research would be useful. The early research on self healing of concrete mainly focused on water retaining structures or reservoirs where leakage through cracks was the main issue (Edvardsen 1999, Reinhardt and Joos 2003). In the research of Ter Heide et al. (2005) and Granger et al. (2006) the main focus was regaining mechanical properties of cracks in early age concrete by ongoing hydration of cement particles.

Ter Heide (2005) gives a nice overview of different causes of autogenic healing (see Fig. 3), in which a material has already by nature the ability to heal itself. On the other hand, materials can also be designed to have a self healing capacity (Schlangen and Joseph 2008). Then we classify them as autonomic materials, which can again be subdivided in passive and active modes. A passive mode smart material has the ability to react to an external stimulus without the need for human inter-

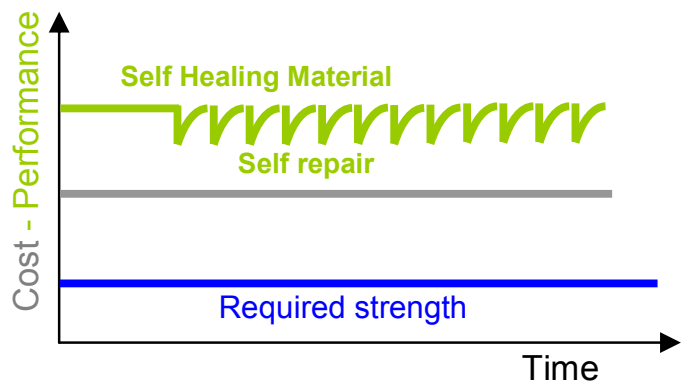

Fig. 2 Performance and cost versus elapse time for structure built with self healing material. Inflation and interest ignored (after van Breugel, 2007).

vention, whereas an active mode smart material or structure requires intervention in order to complete the healing process.

In the last 5 years the design of materials with healing ability is becoming more and more popular in a wide range of materials and applications (Van der Zwaag 2007, Gosh 2008). For cement based materials different method can be found in literature. In the first type of approaches encapsulated sealants or adhesives are used (Dry 200). The adhesives can be stored in short fibres (Li et al. 1998, Qian et al. 2009, van Tittelboom et al. 2011) or in longer tubes (Nishiwaki et al. 2006, Joseph 2008, Joseph et al. 2008). Another approach is incorporating an expansive component in the concrete which starts to expand and fill voids and cracks when triggered by carbonation or moisture ingress (Hosoda et al. 2007, Sisomphon et al. 2009). Using bacteria to stimulate the self healing mechanism is an alternative but promising technique studied at different groups (Bang et al. 2001, Jonkers and Schlangen 2007, De Muynck et al. 2008, Wiktor and Jonkers 2011). More information on the various projects carried out at Delft University can be found on a special Blog that is created (www.selfhealingconcrete.blogspot.com).

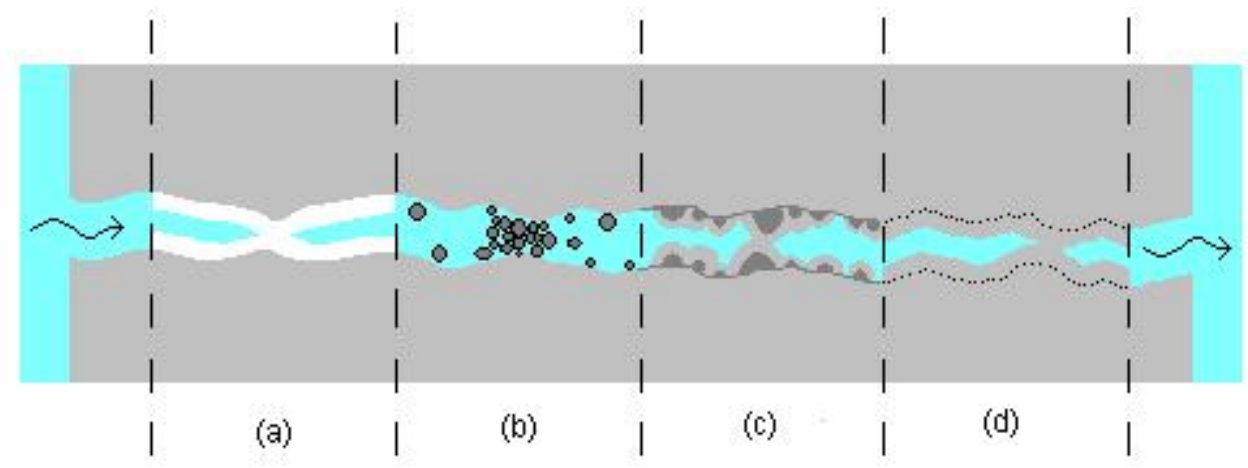

Fig. 3 Possible causes of self healing: (a) formation of calcium carbonate or calcium hydroxide, (b) sedimentation of particles, (c) continued hydration, (d) swelling of the cement-matrix (after Ter Heide 2005). 
At the Microlab of Delft University one of the proposed ideas is to mimic nature by making a novel porous network system in concrete in the form of the spongy part of the bones. This system uses prefabricated thin porous concrete cores which are placed internally in the concrete structure. In the later stage (epoxy-based) healing agents can be transferred through the interconnected pores to reach the damage zone, including micro and macro-cracks, and glue the cracks surface together. Alternatively the healing agent could also be a bacteriacontaining cement paste (Jonkers et al. 2009) or grout, which would make the filling material also self healing when additional cracks appear in future. The goal of the project is to create a self healing material or rather a self healing component in a concrete structure which can tackle many concrete structures problems such as; preventing leakage by forming dense barrier, blocking substance transfer through cracks by crack sealing.

\section{Concept development}

\subsection{Self healing mechanisms in nature and syn- thetic systems}

The route of healing action of synthetic systems can be compared with the biological route as presented in Blaizik (2010). Biological systems respond to injury in three steps, namely inflammatory response (immediate), cell proliferation (secondary), and matrix remodelling (long-term). In more simplistic manner and mostly at accelerated rate, these processes are similarly mimicked by synthetic (biomimetic) system. Damage in material triggers the second response by which self healing agents (SHA) will be transferred into damage location, then, followed by matrix remodelling which is conducted by chemical repair.

Several healing mechanisms in synthetic systems that have been tried successfully namely capsule based, vascular, and intrinsic healing techniques (Blaizik 2010). These techniques have been used for different materials ranging from polymer to ceramic, including concrete.

\subsection{Study of bone morphology and its healing mechanism}

For this research the inspiration comes from the nature of bone and of the complexity of its healing mechanism. Ideas are developed to imitate the process by proposing autonomous repairing mechanisms for concrete.

Structurally, bone can be described as complex hierarchical composite material which consists of cells, fibers, fundamental substances, and different tissues in which collagen is the main structural protein (Balbas 2010). Morphologically bone can be classified into cortical (or compact) bone and cancellous (or trabecular / spongious) bone as shown in Fig. 4.

For the sake of simplicity the complex healing mechanism of fractured bone is described as follows: When bones have fractured as part of surgical procedures or through injury it will demonstrate similar heal- ing response and process. Immediate bleeding and blood clotting at the fracture site provides the initial framework for the next step and inflammation takes place. Then bone production replaces clotted blood with fibrous tissue and cartilage (soft callus) which later on will be replaced by hard callus. The next step is bone remodelling by which tissues become compact and take form returning to its original shape (Kalfas 2001).

\subsection{Mimicking bone healing in concrete struc- tures}

The new self healing technique for concrete material was proposed by imitating bone morphology, that make use of prefabricated cylinder porous concrete core, which are placed internally in the concrete beam as shown in Fig. 5. The porous network constitutes alternate means for (1) channeling temporary or permanent materials to form a dense layer and (2) distributing healing agent to cracks in the main body.

In general the proposed self healing mechanism concept will be carried out in an autonomous manner. This effort can be tackled by adopting intelligent materials concepts which have three basic requirements of capabilities; sensing, actuating, and adaptive controlling to the environment (Leung 2001).

Figure 6 shows the control scheme in this proposed self healing concrete. Damages in the concrete, e.g.

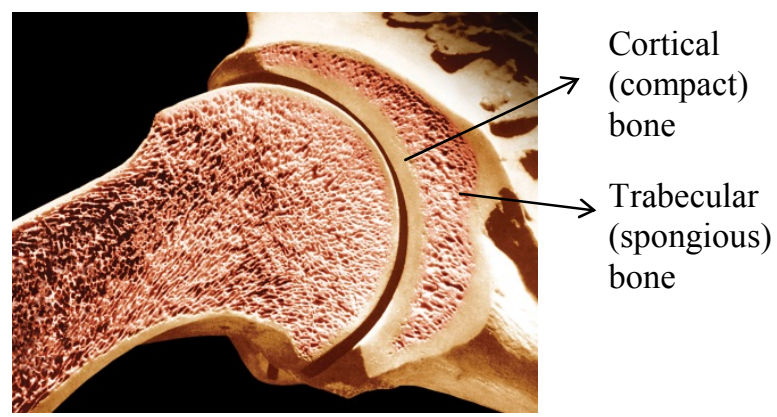

Fig. 4 Longitudinal section of the humerus (upper arm), showing outer compact and inner cancellous (spongy) bone.

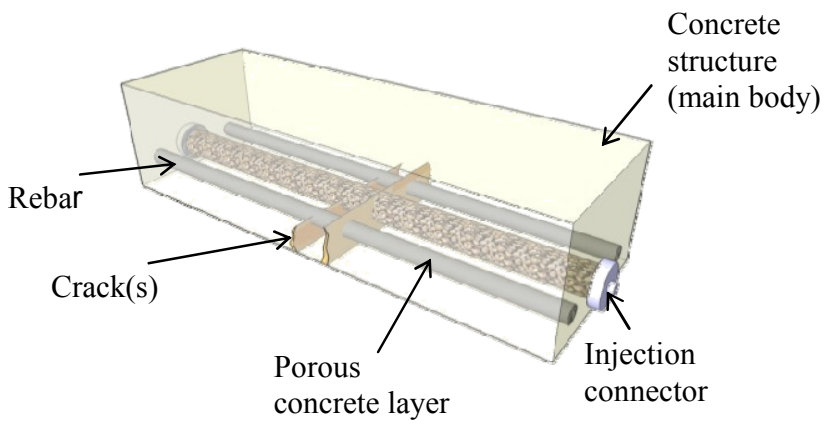

Fig. 5 A conceptual design and application of porous network concrete. 


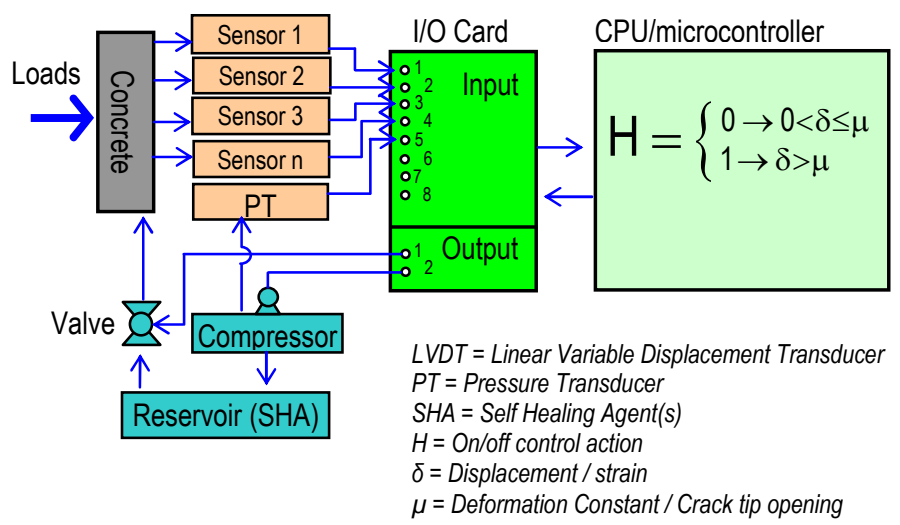

Fig. 6 A design of on-off control scheme for self healing mechanisms in porous network concrete.

cracks located in difficult area for human observation, are detected using sensors. Then data will be collected and calculated by a computer which is then triggers a signal to the actuator. This actuator will switch on a pump that injects healing agent from a reservoir through the porous network concrete layer and makes it dense and also seals cracks. This injection process will be stopped automatically using an algorithm which compares the measured parameters with ultimate or designed values.

\section{General approach and experimental test}

To test the proposed concept in the preliminary phase of the research, the authors designed cylindrical concrete samples. In order to mimic bone structure a porous concrete core was made and placed in the center interior of solid concrete. Uniaxial direct tensile load was applied to create cracks close to the notch in the middle of the sample. Healing action was performed by injecting healing agent manually through the topside injection channel using a syringe. The setup is depicted in Fig. 7.

\subsection{Material design}

To create porous network concrete a porous concrete cylinder was used as a core. Based on the works of many researchers (Yang and Jiang 2002, Mahboub et al. 2009), porous concrete initial mix design was formulated using 2-4 mm single graded aggregate. Weight composition was $1513 \mathrm{~kg} / \mathrm{m}^{3}$ gravel, $355 \mathrm{~kg} / \mathrm{m}^{3}$ ordinary Portland cement CEM I 42.5, $22 \mathrm{~kg} / \mathrm{m}^{3}$ Pulverized Fly Ash (PFA) and $1.41 / \mathrm{m}^{3}$ super-plasticizer with 0.28 water/cement ratio.

Porous concrete cylinders of $\varnothing 35 \mathrm{~mm}$ with $130 \mathrm{~mm}$ height were casted in PVC mould and compacted by pressing and top vibrating. After casting all samples were covered with plastic. In 24 hour samples were demoulded and cured in curing chamber $\left( \pm 20^{\circ} \mathrm{C}, 95 \% \mathrm{RH}\right)$. After seven days the samples were taken out of the curing chamber and allowed to achieve saturated surface dry (SSD) condition for 24 hours (see Fig. 8).

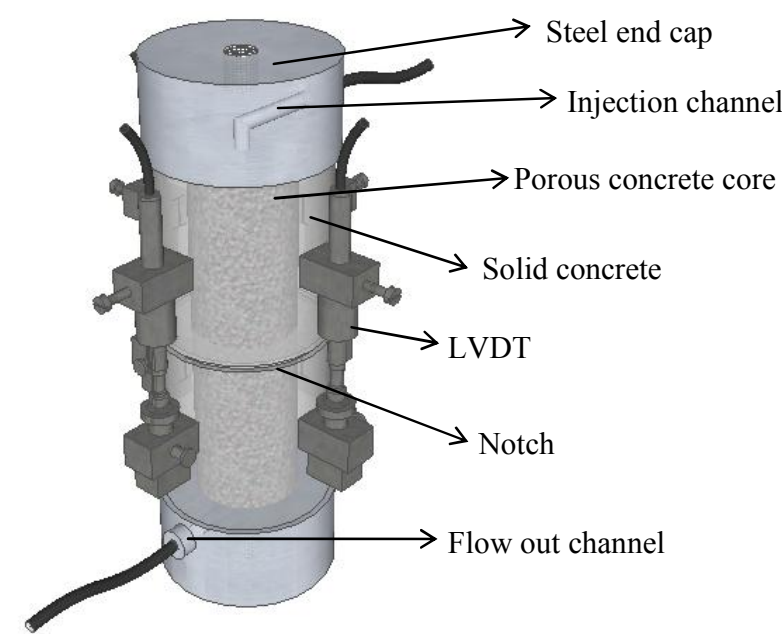

Fig. 7 Conceptual design of material and method of proposed healing action.

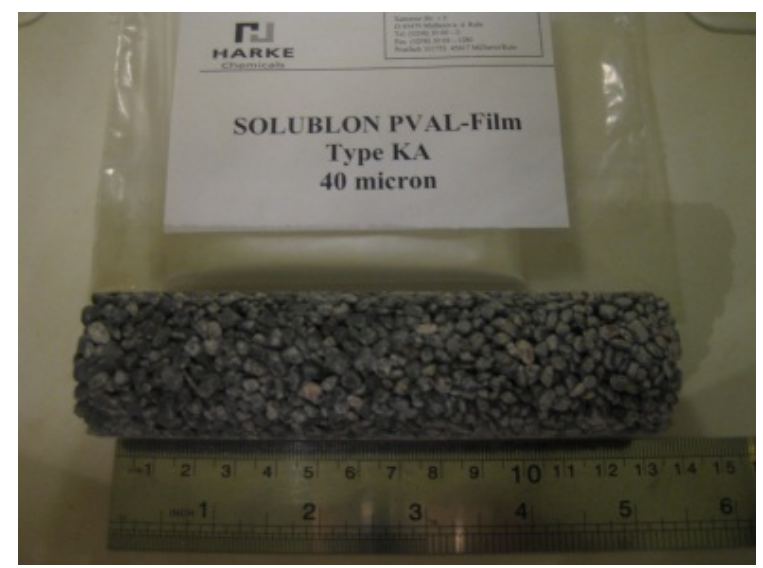

Fig. 8 Porous concrete cynlider that would be used as porous core in PNC samples.

Achieving SSD, one sample was covered with PVA water soluble plastic and one sample was not covered. A cold water soluble plastic, SOLUBLON PVAL-film grade KA 40 micron supplied by HARKE Chemical 

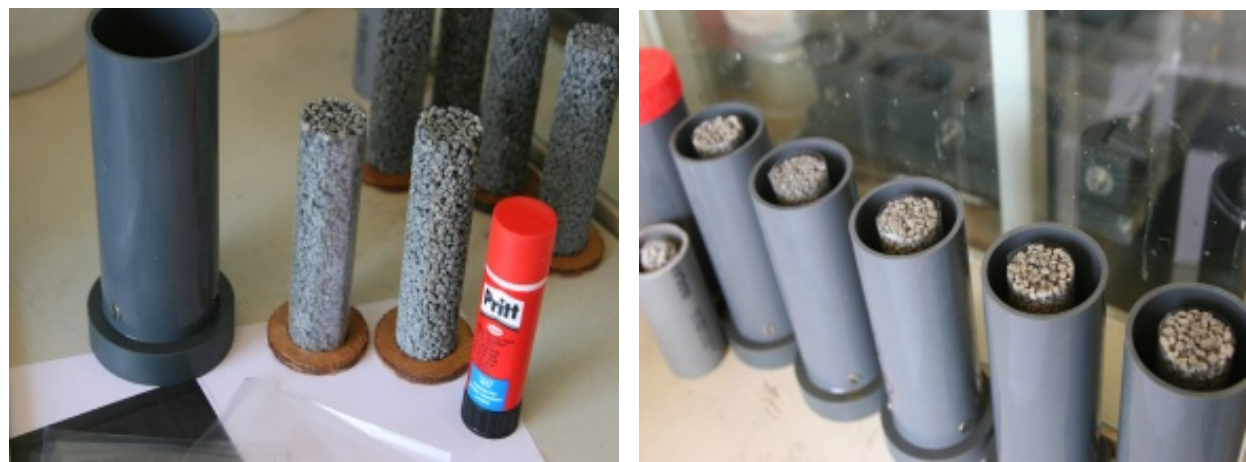

Fig. 9 Casting preparation where porous concrete cylinder was placed in the center of the mould and normal strength self compacting concrete was poured around.

$\mathrm{GmbH}$ was used in this experiment.

Afterwards the porous cylinder was put in the center of a $\varnothing 56 \mathrm{~mm}$ PVC mould as shown in Fig. 9. Medium strength self compacting concrete designed based on the work of Mohammed (2004), was used as outer solid concrete and casted around the porous cylinder core. The samples are treated with similar curing procedure as explained above for next 7 days.

Figure 10 shows porous network concrete, a new hierarchical material that has been developed in which pore connection can be used as media for transportation

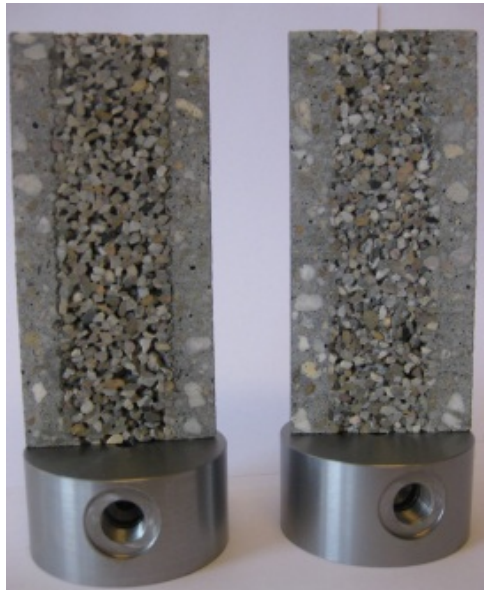

Fig. 10 Bone-like concrete; a new hierarchical material is made in which sponge-like core is surrounded by solid concrete. of healing agents. Boundary between porous core and solid concrete was more obvious in the samples in which the porous core was covered with PVA film resulting in more regular circle core while an irregular boundary can be seen in samples without PVA film cover as shown in Fig. 11.

\subsection{Creation of crack}

At an age of 7 days, porous network concrete samples were taken out from the curing chamber and dried in an oven at $35^{\circ} \mathrm{C}$ for 24 hours. Then, tensile stress was applied to create a crack in the notch region in the middle of the sample height (see Fig. 12a). The test has been done in deformation control at the rate of $0.1 \mu \mathrm{m}$ per second until a displacement of about $200 \mu \mathrm{m}$ was reached. Plastic sheets were placed in the top and bottom side centre of the samples to avoid glue contact between the porous core and steel end clamps, so tension was isolated to the solid concrete.

\subsection{Crack healing by manual injection}

At a crack opening of $200 \mu \mathrm{m}$ the tensile load was removed. Then the samples were taken out of the instrument and healing agent was injected using syringe through the top side end cap as can be seen in the Fig. $12 b$.

Epoxy was chosen as healing agents explicitly to seal the crack (Schlangen and Joseph 2009, Issa and Debs 2007). The healing agent consists of epoxy resin Conpox Harpiks BY 158 (liquid) and hardener Haerder HY
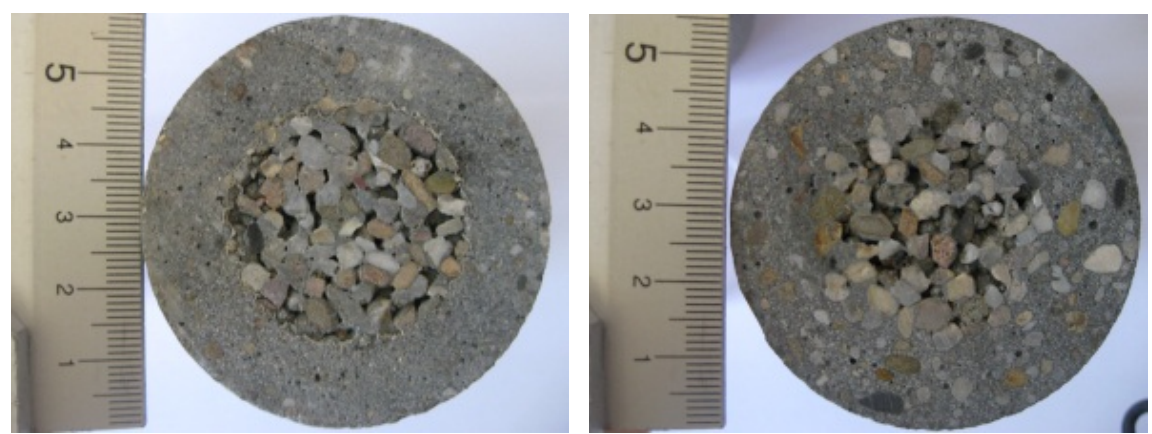

Fig. 11 a) Left; more regular circle of porous core due to PVA film cover, and b) Right: irregular boundary due to penetration of cement paste into uncovered porous core. 

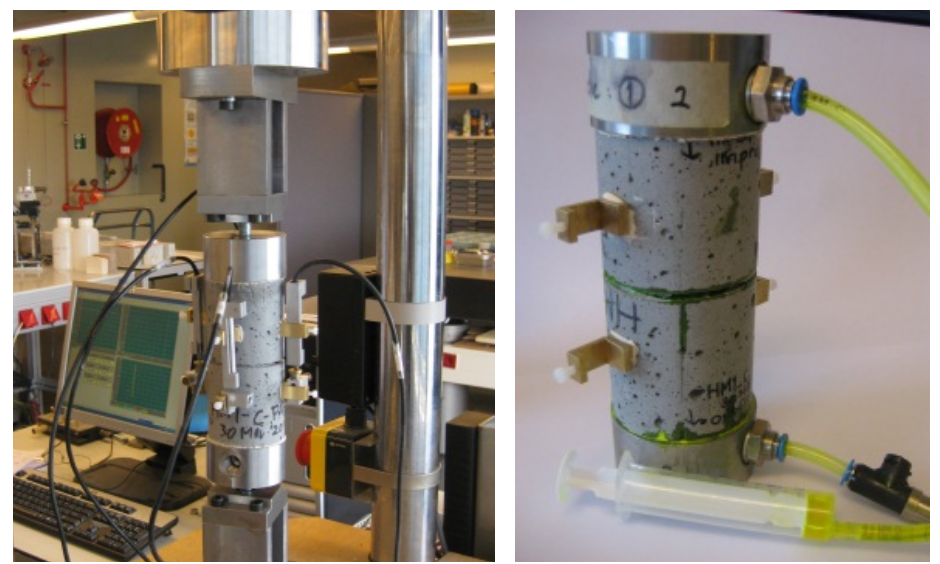

Fig. 12 a) Left; Deformation controlled tensile test. b) Right; SHA was manually injected into porous layer at the top side.

\section{Load vs displacement (avg) diagram before \& after injection SHA}

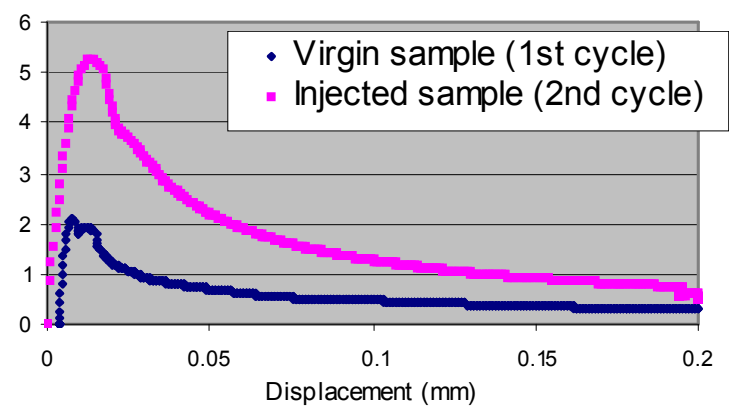

Fig. 13 Deformation controlled tensile test.

2996 (liquid) with weight ratio of 0.3 . Fluorescent dye (powder) is used with $1 \%$ weight proportion to epoxy to help visualize pore and cracks under Ultra Violet (UV) light. After the injection process the samples were kept in the oven at $\pm 35^{\circ} \mathrm{C}$ for 24 hour. This process is carried out to ensure epoxy polymerization has taken place completely. After complete polymerization, one of the samples was tested in a second cycle under tensile loading.

\subsection{Visualization of crack healing}

One sample was cut longitudinally (vertical) to see how epoxy fills pore spaces and cracks. Under UV light the longitudinal section of the samples was portrayed. An other method of visualization applied in this research is putting a sample in the X-ray $\mu \mathrm{CT}$ Scanner. 3D image reconstruction has been done using ImageJ to process image stacks, DeVIDE to reconstruct 3D image, and MeshLab to visualize the image produced as shown in Fig. 16b.

\section{Results and discussions}

Some tendencies have been recognized, although there is certainly some variability in the results obtained from the experiments due to the heterogeneous nature of the

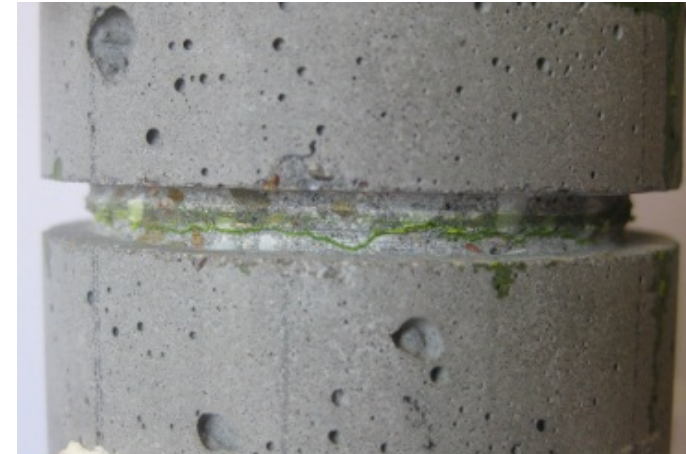

Fig. 14 Crack formation of concrete cylinder in the notch.

system investigated. The average load-displacement response of the cylinder tested is presented in Fig. 13.

It may be seen that for virgin samples there is a peak value of tensile load around $2.2 \mathrm{kN}$. It is noticed that the peak tensile load value occurs when the crack mouth opening displacement (CMOD) reach $15 \mu \mathrm{m}$, followed by non-linear softening behavior until CMOD reached $200 \mu \mathrm{m}$ when the test was stopped. Figure 14 visually confirms crack formation in the notch area of the cylinder.

The efficacy of the manually assisted healing action of porous network concrete may be examined by comparing the mechanical response of the healed cylinder to the initial response of the virgin cylinder. The second loading cycle results in a similar load-CMOD response, but with higher peak value approximately $5.2 \mathrm{kN}$ at 25 $\mu \mathrm{m}$ crack width. It may be noted that also a higher material stiffness in the linear elastic phase has been obtained which is illustrated by the diagram of the injected sample.

This apparent 'enhancement' of response in term of higher value of initial stiffness and peak tensile load occurs due to the following reasons: The low viscosity epoxy is believed flow and fills up all void spaces in the porous concrete core including crack in the fracture process zone (FPZ), hence, creating a polymercementitious composite action which enhances the mechanical properties in the cylinder. 

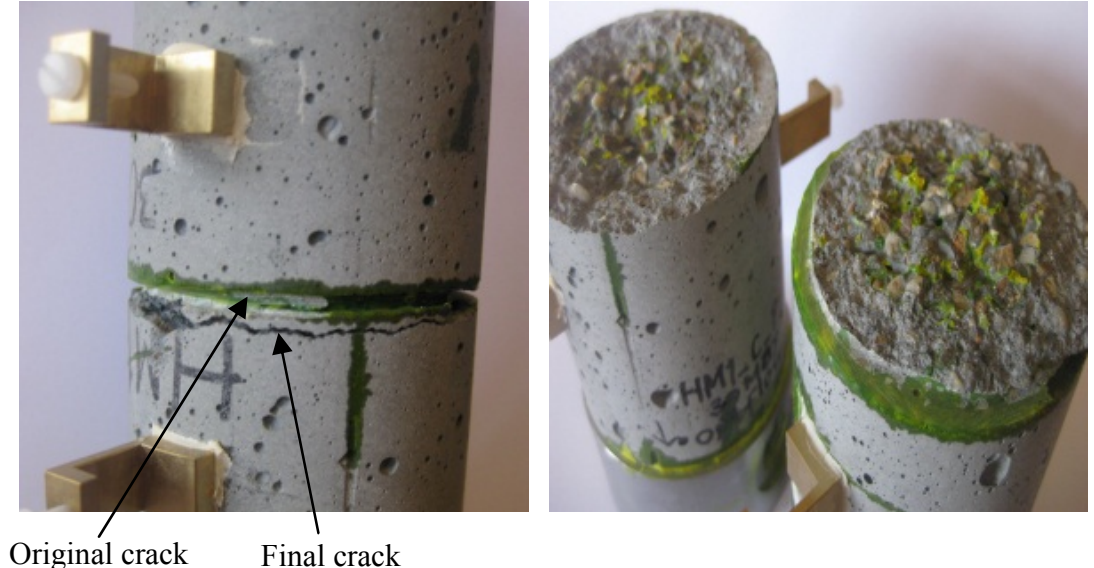

Original crack Final crack

Fig. 15 Original and final crack pattern and new crack surface.
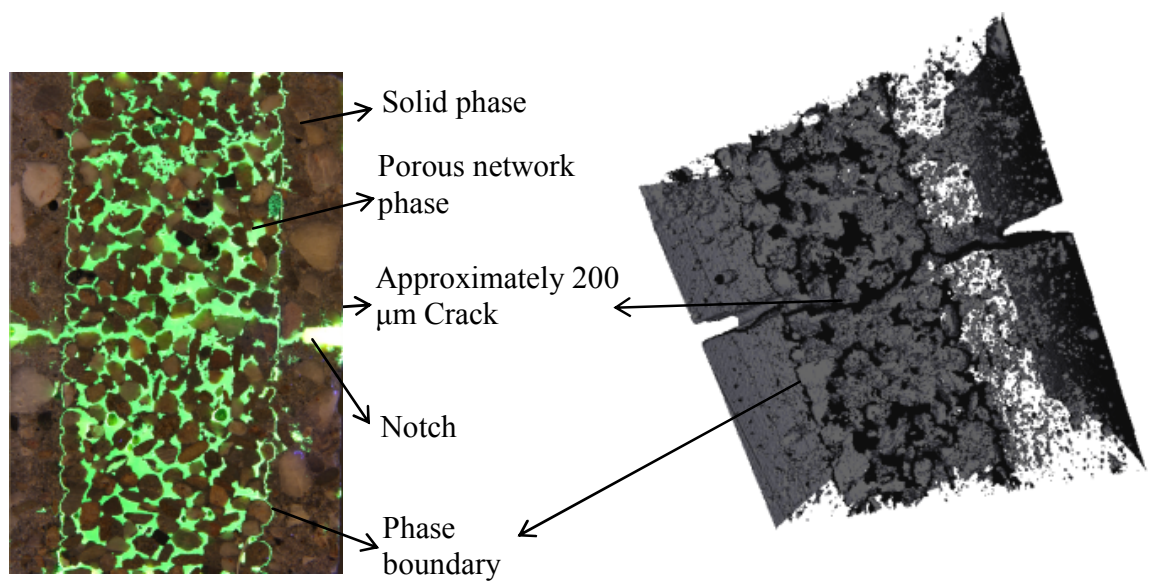

Fig. 16 (a) Longitudinal cross section showing the crack which has been filled by epoxy. (b) 3D reconstruction of the vascular concrete after crack propagation.

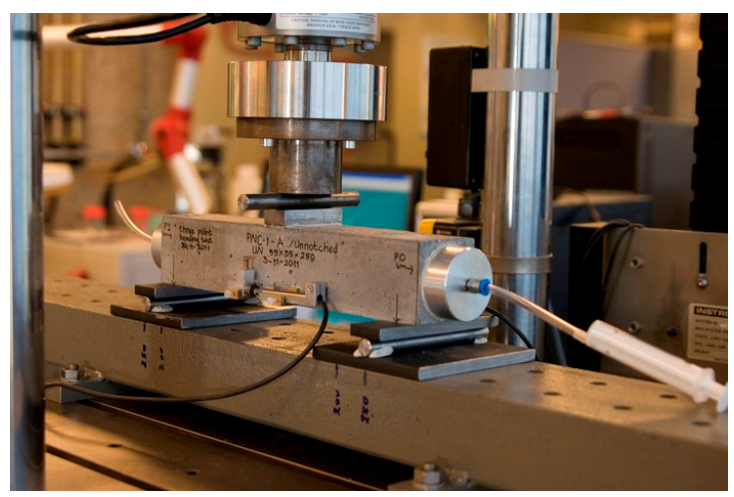

Fig. 17 Three points bending test for crack formation showing injection connector and LVDT.

Visual confirmation of healed response is provided by a new crack surface formation which occurred in the cylinder. Figure 15 shows the original and final crack patterns on the side face of the cylinder and new fracture surface that shifted some millimeter away from the notched area where the previous crack was formed. It is clear from Fig. 15 that in spite of the concentration of the stress built up by the notch that created the original macro-crack during the first loading cycle, the final crack occurred at a different location for the self-healing specimens. The crack at this new location was not observed to occur in the first cycle, and therefore, this is clear evidence of the effectiveness of the bonding capabilities of the epoxy when used within a concrete.

Bright green epoxy polymer can be seen filling up all space including crack in the fracture process zone of the sample (see Fig. 16). It may be noticed that the boundary line between solid phase and porous concrete is visible and filled with epoxy. It can be concluded that PVA film was dissolved during or after casting the self compacting concrete. This phenomenon ensured that the porous concrete could be kept porous in the interior of the concrete structure.

Furthermore reinforced concrete prisms were tested as presented in the conceptual design sketched in Fig. 5. The beams have a size 55 × 55 × $295 \mathrm{~mm}$. One Ø3-295 $\mathrm{mm}$ threaded steel rebar was placed longitudinally in the centreline of the beam $10 \mathrm{~mm}$ from the bottom face. A porous cylinder of Ø26-295 $\mathrm{mm}$ was put on top of the rebar. The beams were produced in the same way as the cylindrical specimens discussed before.

The strain controlled three point loading test was performed with strain rate of $0,1 \mathrm{~mm} / \mathrm{sec}$ as shown in Fig. 


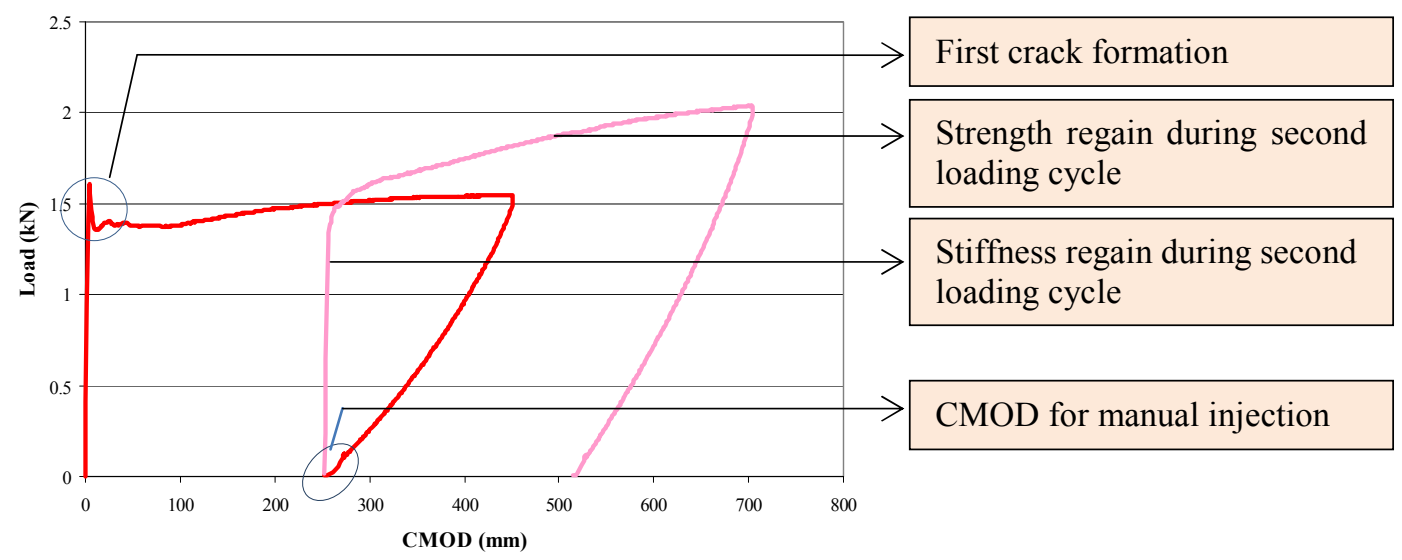

Fig. 18 Load versus CMOD of porous network concrete with second loading after the crack was injected with healing agents through porous network in the interior of concrete main body.
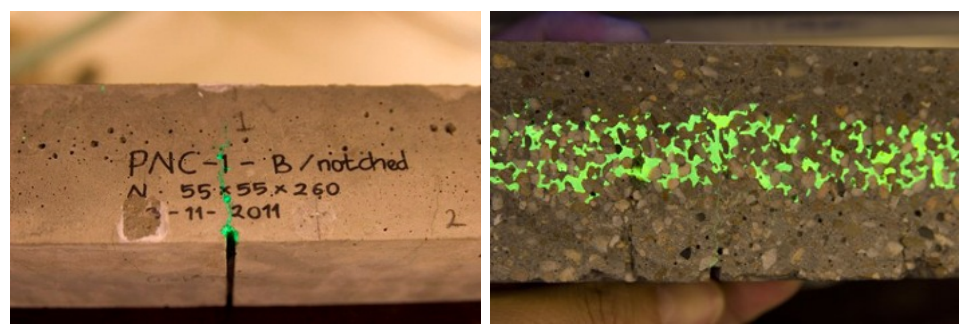

Fig. 19 (a) Original crack formation filled up with epoxy and (b) final crack pattern from second cycle loading has shifted from previous crack plane.

17. The beam was simply supported by steel cylinder with $250 \mathrm{~mm}$ span and the load was right in the midspan. To control and measure the crack width during the test a linear variable differential transformers (LVDT) which has a range of $\pm 500 \mu \mathrm{m}$ with an accuracy of 1 $\mu \mathrm{m}$ was attached to the beam, one on each side of the beam specimen at the bottom.

After the first crack had been created in these beams of approximately $400 \mu \mathrm{m}$, the load was removed and the crack mouth opening decreased to a value of approximately $250 \mu \mathrm{m}$. At this points the injection of healing agent was carried out through the porous core by means of an manual injection. The automatic injection action with a system as proposed in Fig. 5 was still under construction. To achieve complete polymerization of healing agents 24 hours curing time was allowed in the oven at $35^{\circ} \mathrm{C}$. Afterwards the beams were loaded again to measure the strength after healing and to observe crack development. The strength and stiffness was regained (see Fig. 18) completely and new cracks developed as can be seen in Fig. 19, where the original cracks are shown as well as the newly developed cracks in the second loading cycle.

\section{Concluding remarks}

In this article a new approach of self healing that makes use of a porous network concrete is described. This rather innovative idea mimics bone shape material and its healing process when injury happens. Prefabricated porous concrete cylinders were place internally in the centre of concrete cylinder structures, which is somewhat similar to bone in terms of morphology. Manual healing intervention at the right time and location can be done effectively. A method is proposed to turn the manual healing onto a completely automated self healing system. In addition, the authors consider that self healing agent (SHA) e.g. chemical-based, bacteria containing liquid, or cement slurry can be chosen depending on the application criteria in the practical situation.

Comparing both mechanical responses between virgin and healed samples shows clear evidence that healing has taken place using the proposed porous network concrete. The effectiveness of the novel approach is also confirmed by visual evidence provided.

This method of self-healing or automatic repair is extremely suitable for situation that are difficult to repair from the outside, for instance when the cracks are not accessible or in situations where it is too dangerous to do a manual repair.

At this moment the on-going research is focussing on determining over which length injection is possible and how many injection points and parallel tubes are necessary to perform self-healing in practical situations and also be able to perform multiple self-healing events.

\section{Acknowlegement}

Mr. Gerrit Nagtegaal and Mr. Arjan Thijssen were very 
instrumental in supporting the author for mechanical test and X-ray $\mu \mathrm{CT}$ scanning, respectively. Furthermore, the authors acknowledge the support of HARKE chemical GmbH Germany for supplying PVA film for the experiments.

\section{References}

Balbas, E. M., (2001). “Optical Techniques for the Study of Living Tissue." Doctoral Thesis Delft: Technische Universtiteit Delft.

Bang, S. S., Galinat, J. K. and Ramakrishnan, V., (2001). Calcite precipitation induced by polyurethaneimmobilized Bacillus pasteurii. Enzyme Microb. Technol. 28, 404-409.

Blaiszik, B. J., Kramer, S. L. B., Olugebefola, S. C., Moore, J. S., Sottos, N. R. and White S. R., (2010). "Self-Healing Polymers and Composites." Annual Review of Materials Research, 40, 179-211.

De Muynck, W., Debrouwer, D., De Belie, N. and Verstraete, W., (2008). "Bacterial carbonate precipitation improves the durability of cementitious materials." Cement and Concrete Research, 38, 10051014.

Dry, C. M., (2000). "Three designs for the internal release of sealants, adhesives, and waterproofing chemicals into concrete to reduce permeability." Cement and Concrete Research, 30(12), 1969-1977.

Edvardsen, C., (1999). "Water permeability and autogenous healing of cracks in concrete." $A C I$ Materials Journal, 96(4), 448-454.

Granger, S., Loukili, A., Pijaudier-Cabot, G. and Behloul, M., (2006). "Self healing of cracks in concrete: from a model material to usual concretes." In: Proceedings of the 2nd international symposium on advances in concrete through science and engineering. Quebec city, Canada, 11-13 September 2006.

Gosh, S. K. ed., (2008). "Self-healing materials; fundamentals, design strategies and applications." Wiley.

Hosoda, A, Kishi, T., Arita, H. and Takakuwa, Y., (2007). "Self healing of crack and water permeability of expansive concrete." In: 1st international conference on self-healing materials. Noordwijk, Holland.

Issa, C. A. and Debs, P. "Experimental study of epoxy repairing of cracks in concrete." Construction and Building Materials, vol. 21, pp. 157-163, 2007.

Jonkers, H., Schlangen, E., Schmets, A. J. M. and van der Zwaag, S. eds., (2007). Proceedings of the First International Conference on Self Healing Materials, 18-20 April 2007, Noordwijk aan Zee, The Netherlands, Springer.

Jonkers, H. M., Thijssen, A., Muijzer, G., Copuroglu, O. and Schlangen, E., (2009). "Application of bacteria as self-healing agent for the development of sustainable concrete." Ecological engineering, 1-6.

Joseph, C., (2008). "Experimental and numerical study of fracture and self healing of cementitious materials." PhD thesis, Cardiff University.

Joseph, C., Jefferson, A. D. and Lark, R. J., (2008). "Lattice modelling of autonomic healing processes in cementitious materials." In: WCCM8 / ECCOMAS 2008, Venice, Italy.

Kalfas, I. H., (2001). "Principles of bone healing." Neurosurgical Focus, 10, 04/01/2001.

Li, V. C., Lim, Y. M. and Chan, Y. W., (1998). "Feasibility study of a passive smart self-healing cementitious composite." Composites Part B: Engineering, 29(B), 819-827.

Leung, C. K. Y., (2001). "Fiber optic sensors in concrete: the future?" NDT \& E International, 34, 85-94.

Mahboub, K. C., Canler, J., Rathbone, R., Robl, T. and Davis, B., (2009). "Pervious Concrete: Compaction and Aggregate Gradation." ACI Materials Journal, 106, 523-528.

Mohammed, S., (2004). "Medium strength selfcompacting concrete containing fly ash: Modelling using factorial experimental plans." Cement and Concrete Research, 34, 1199-1208.

Neville, A., (2002). "Autogenous healing - A concrete miracle?" Concrete International, November 2002.

Nishiwaki, T., Mihashi, H., Jang, B-K. and Miura, K., (2006). "Development of self-healing system for concrete with selective heating around crack." Journal of Advanced Concrete Technology, 4(2), 267275.

Qian, S., Zhou, J., de Rooij, M. R., Schlangen, E., Ye, G. and van Breugel, K., (2009). "Self-Healing Behavior of Strain Hardening Cementitious Composites Incorporating Local Waste Materials." Cement and Concrete Composites, 31, 613-621.

Reinhardt, H. W. and Joos, M., (2003). "Permeability and self-healing of cracked concrete as a function of temperature and crack width." Cement and Concrete Research, 33(7), 981-985.

Schlangen, E. and Joseph, C., (2008). "Self-healing processes in concrete." In: SK Ghosh (Ed.), Selfhealing materials: fundamentals, design strategies and applications, Weinheim: Wiley, 141-182.

Sisomphon, K., Çopuroğlu, O. and Fraaij, A. L. A., (2009). "Durability of Blast-Furnace Slag Mortars Subjected to Sodium Monofluorophosphate Solution Curing." In: Proc. 4th International Conference on Construction Materials: Performance, Innovations and Structural Implications, Nagoya, Japan.

Ter Heide, N., (2005). "Crack healing in hydrating concrete." MSc-thesis, Delft University of Technology, The Netherlands.

Ter Heide, N., Schlangen, E. and van Breugel, K., (2005). "Experimental Study of Crack Healing of Early Age Cracks.” In: Proceedings Knud Højgaard conference on Advanced Cement-Based Materials, Technical University of Denmark, June 2005.

Van Breugel, K., (2007). "Is there a market for selfhealing cement-based materials? "1st international 
conference on self-healing materials. Noordwijk, Holland, 2007.

Van der Zwaag, S. (ed), Self healing materials : an alternative approach to 20 centuries of materials science, Dordrecht, Netherlands, Springer, 2007.

Van Tittelboom, K., De Belie, N, Van Loo, D. and Jacobs, P., (2011). "Self-healing efficiency of cementitious materials containing tubular capsules filled with healing agent." Cement and Concrete Composites, 33(4), 497-505.
Wiktor, V. and Jonkers, H. M., (2011). "Quantification of crack-healing in novel bacteria-based self-healing concrete." Cement and Concrete Composites, 33, 763-770.

www.selfhealingconcrete.blogspot.com [Accessed 29 February 2012]

Yang J. and Jiang, G., (2002). "Experimental study on properties of pervious concrete pavement materials." Cement and Concrete Research, 33, 381-386. 


\title{
Scientific paper
}

\section{Experimental Study on Self-Healing Capability of FRCC Using Different Types of Synthetic Fibers}

\author{
Tomoya Nishiwaki ${ }^{1}$, Marina Koda ${ }^{2}$, Makoto Yamada $^{2}$, Hirozo Mihashi ${ }^{3}$ and Takatsune Kikuta ${ }^{4}$
}

\begin{abstract}
Experimental studies are carried out to evaluate the self-healing capability of FRCC using different types of synthetic fibers that have different chemical properties, i.e. poly vinyl alcohol (PVA), ethylene vinyl alcohol (EVOH), polyacetal (POM) and polypropylene (PP). FRCC specimens were subjected to tension tests in order to generate a crack, and the cracked specimens were immersed in water. In order to evaluate the effect of self-healing phenomena, permeability tests and microscopic observation were carried out. Microscopic observation revealed that the high polarity of synthetic composite has high potential of self-healing precipitation around fibers bridging a crack. Moreover, the coefficient of water permeability was generally reduced with this chemical precipitation, especially in the PVA series. However, even when it was confirmed by microscopic observation that precipitation had appeared and filled a crack, we found there is no recovery of water tightness in some cases, i.e. in the EVOH and POM series. It is confirmed that not only the chemical properties of fibers but also the geometrical properties of the crack surface, such as roughness, complexity and continuity, affect the capability of self-healing for water tightness.
\end{abstract}

\section{Introduction}

To maintain the global environment and build a sustainable society, highly durable and long-life concrete structures are strongly required. However, cracking is inherent in concrete structures and it leads to serious damage that cause durability issues, e.g. increase in permeability, ingress of aggressive agent and corrosion of reinforcing steel. It is important to perform periodic and/or detailed inspection, and implement proper repairing procedures for preventing such damage and deterioration. On the other hand, even in the case of ordinary concrete, cracks with small width of $0.1 \mathrm{~mm}$ or less tend to close autogenously in the presence of moisture due to precipitation of calcium carbonate (Edvardsen 1999). A number of studies have been carried out to enhance the self-healing capability of concrete using several approaches. Reinhardt and Joose (2003) revealed that curing temperature has a great influence on self-healing of cracked concrete. Ahn and Kishi (2010) applied some types of mineral admixtures and Jonkers et al. (2010) demonstrated quite a novel approach using bacteria as a self-healing agent in order to enhance self-healing capability. Such crack closing phenomena are useful for preventing invasion of aggressive agent,

\footnotetext{
${ }^{1}$ Associate Professor, Graduate School of Engineering, Tohoku University, Sendai, Japan.

E-mail: ty@archi.tohoku.ac.jp

${ }^{2}$ Tohoku University, Graduate School of Engineering, Sendai, Japan.

${ }^{3}$ Professor emeritus, Tohoku University, Sendai, Japan.

${ }^{4}$ Assistant Professor, Graduate School of Engineering, Tohoku University, Sendai, Japan.
}

though efficient recovery of strength is doubtful.

In Japan, a technical committee of the Japan Concrete Institute (JCI) summarized recent research on self-healing concrete, giving definitions of various types of such concrete (Igarashi et al. 2009). Per these definitions, enhancing methods that use admixtures are categorized as "autonomic healing," i.e. involuntary healing of cracks by admixtures. Fiber reinforced cementitious composite (FRCC) is one of the autonomic healing materials. FRCC has mechanical characteristics that can control crack propagation in the cement matrix through bridging with short fibers. Since the self-healing process requires crack widths that are sufficiently small, FRCCs are expected to have great self-healing capability. Li et al. (1998) carried out experimental studies on the self-healing capability of Engineered Cementitious Composites (ECC) and concluded that cementitious materials with inherently tight crack width effectively self-heal. Kan et al. (2010) identified self-healing products of ECC as mainly C-S-H and calcium carbonate through ESEM-EDS and TEM observation. Qian et al. (2009) showed the self-healing behavior of fiber reinforced strain hardening cementitious composites incorporating blast furnace slag and limestone powder. The recovery in deflection capacity and stiffness was found to range between $65 \%$ and $105 \%$ in virgin specimens, significantly higher compared to the specimens without benefit of the self-healing process. Mihashi et al. (2011) declared FRCC to have better resistance against corrosion of steel bars than mortar because of the fiber bridging of cracks and the self-healing of some of the cracks. Homma et al. (2009) confirmed that fibers bridging the crack surface play a role not only as a crack width controller but also as a core for depositing chemical products, and that a larger number of bridging 
fibers achieves more effective self-healing. Koda et al. (2011) concluded that different types of fibers show different levels of self-healing performance. In particular, fibers with polarity (e.g. PVA) promote more effectively the deposition of crystallization products than other types of fibers.

In this study, the self-healing capability of FRCCs containing synthetic fibers with different chemical properties is investigated to reveal the role of the fibers as a core for the precipitation of calcium carbonate and to enhance the potential of the self-healing function of FRCC.

\section{Self-healing mechanism}

Self-healing is a natural process of crack repair that can occur in concrete in the presence of moisture. The deposition of calcium carbonate is said to occur as the result of the following reactions (Edvardsen 1999).

$$
\begin{aligned}
& \mathrm{H}_{2} \mathrm{O}+\mathrm{CO}_{2} \leftrightarrow \mathrm{H}_{2} \mathrm{CO}_{3} \leftrightarrow \mathrm{H}^{+}+\mathrm{HCO}_{3}{ }^{-} \leftrightarrow 2 \mathrm{H}^{+}+\mathrm{HCO}_{3}{ }^{2-} \\
& \mathrm{Ca}^{2+}+\mathrm{CO}_{3}{ }^{2-} \leftrightarrow \mathrm{CaCO}_{3}\left(\mathrm{pH}_{\text {water }}>8\right) \\
& \mathrm{Ca}^{2+}+\mathrm{HCO}_{3}{ }^{-} \leftrightarrow \mathrm{CaCO}_{3}+\mathrm{H}^{+}\left(7.5<\mathrm{pH}_{\text {water }}<8\right)
\end{aligned}
$$

The dissolved $\mathrm{CO}_{2}$ ion and the calcium ion $\mathrm{Ca}^{2+}$ in water combine with each other to produce calcium carbonate crystals, and the resulting calcium carbonate crystallization is precipitated on the crack surface. As a result, the crack width is gradually reduced and ultimately the crack closes. Homma (2009) revealed also that the self-healing mechanism was accelerated in the crack with bridging fiber. Polymers that have polarity can attract $\mathrm{Ca}^{2+}$ and become a template of calcium carbonate (Kato et al. 2008). For that reason, some types of synthetic fibers, such as PVA, can attract $\mathrm{Ca}^{2+}$ and promote the deposition of crystallization products.

\section{Testing procedures}

In this study, two experiments (hereafter called A and B) were carried out to evaluate the self-healing capability of FRCC using different types of synthetic fibers. In experiment $\mathrm{A}$, the volume of the precipitated crystals of calcium carbonate around each fiber that was exposed from the crack surface of FRCC plates was measured to estimate the potential of self-healing. In experiment B, microscopic observation of the crack surface and a water permeability test on FRCC plates containing different types of fibers were carried out to evaluate the self-healing capability.

\subsection{Experiment $A$ (observation of precipitated crystals around each fiber)}

The precipitation of crystals of calcium carbonate around each fiber exposed from the crack surface of FRCC immersed in curing water was observed and the volume of the precipitation was measured. Table 1 lists the mix proportion of the FRCC specimens used in experiment A and experiment B. Tables $\mathbf{2}$ and $\mathbf{3}$ gives the properties of the employed synthetic fibers and the other materials, respectively. Figure 1 shows the chemical constitution of the fibers, with the circles indicating polarity groups. Polarity strengths depend on electro-negativity, which is the tendency of an atom or a functional group to attract electrons. In Fig. 1, PVA has the highest polarity strength due to the $\mathrm{OH}$ radical, and PP has no polarity strength. EVOH consist of both PVA and PP parts, and POM has the $\mathrm{O}$ radical. Thus, the polarity strengths of EVOH and POM are of moderate level between PVA and PP. Prismatic specimens of $40 \mathrm{~mm} \times 40 \mathrm{~mm} \times 160 \mathrm{~mm}$ were prepared. After standard curing for 7 days, each specimen was subjected to the 3-point bending test and completely separated. A half piece of specimen was cut into

Table 1 Mix proportion of FRCC specimens.

\begin{tabular}{|c|c|c|c|c|}
\hline W/B & S/B & SF/B & SP/B & $\begin{array}{c}\text { Fiber } \\
\text { (Vol. \%) }\end{array}$ \\
\hline 0.45 & 0.45 & 0.15 & 0.009 & 2.0 \\
\hline
\end{tabular}

B: binder (cement + silica fume)

Table 3 Properties of employed materials.

\begin{tabular}{|c|c|c|c|c|c|c|}
\hline Series & Type of Fiber & $\begin{array}{l}\text { Density } \\
{\left[\mathrm{g} / \mathrm{cm}^{3}\right]}\end{array}$ & $\begin{array}{c}\text { Tensile } \\
\text { Strength } \\
{\left[\mathrm{N} / \mathrm{mm}^{2}\right]}\end{array}$ & $\begin{array}{c}\text { Length } \\
{[\mathrm{mm}]}\end{array}$ & $\begin{array}{l}\text { Diameter } \\
{[\mu \mathrm{m}]}\end{array}$ & $\begin{array}{l}\text { Shape of } \\
\text { Section }\end{array}$ \\
\hline PP & \multirow{2}{*}{ Polypropylene } & \multirow{2}{*}{0.91} & \multirow{2}{*}{760} & \multirow{2}{*}{6} & 11 & Circle \\
\hline C-PP & & & & & 18 & Deformed \\
\hline POM & Polyacetal & 1.41 & 135 & 10 & 48 & Circle \\
\hline $\mathrm{EVOH}$ & $\begin{array}{l}\text { Ethylene vinyl alcohol } \\
\text { copolymer }\end{array}$ & 1.04 & 231 & 5 & 15 & Circle \\
\hline PVA-I & \multirow{2}{*}{ Polyvinyl alcohol } & \multirow{2}{*}{1.30} & \multirow{2}{*}{1600} & \multirow{2}{*}{6} & 14 & \multirow{2}{*}{ Circle } \\
\hline PVA-II & & & & & 37 & \\
\hline
\end{tabular}

\begin{tabular}{|c|c|l|}
\hline Material & Symbol & \multicolumn{1}{|c|}{ Properties } \\
\hline Cement & C & $\begin{array}{l}\text { High early strength Portland } \\
\text { cement Density: } 3.14 \mathrm{~g} / \mathrm{cm}^{3}\end{array}$ \\
\hline $\begin{array}{c}\text { Silica } \\
\text { fume }\end{array}$ & SF & Density: $2.20 \mathrm{~g} / \mathrm{cm}^{3}$ \\
\hline Sand & S & $\begin{array}{l}\text { Silica sand } \# 5, \text { Density: } 2.61 \\
\text { g/cm } / 3 \text { Diameter: } \sim 500 \mu \mathrm{m}\end{array}$ \\
\hline $\begin{array}{c}\text { Super } \\
\text { plasticizer }\end{array}$ & SP & $\begin{array}{l}\text { Polycarboxylic acid ether system } \\
\text { Density: } 1.05 \mathrm{~g} / \mathrm{cm}^{3}\end{array}$ \\
\hline
\end{tabular}

Table 2 Properties of employed fibers. 


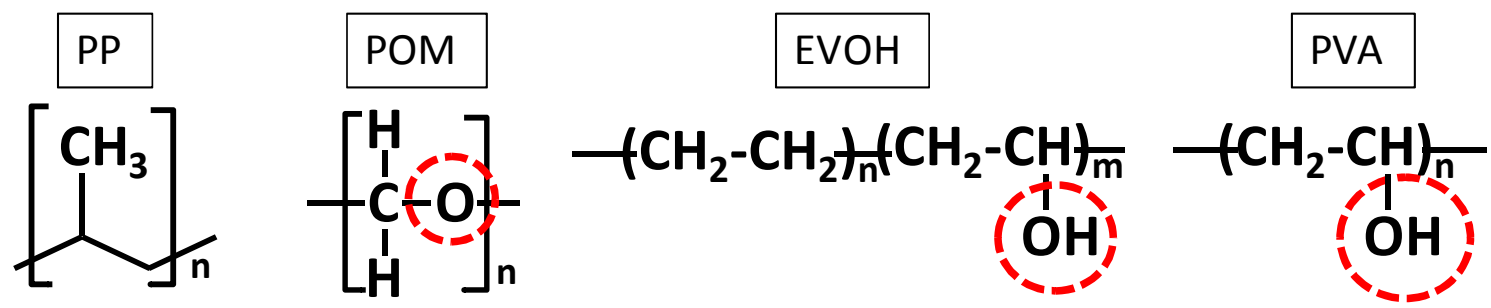

Fig. 1 Characteristic parts of chemical constitution.

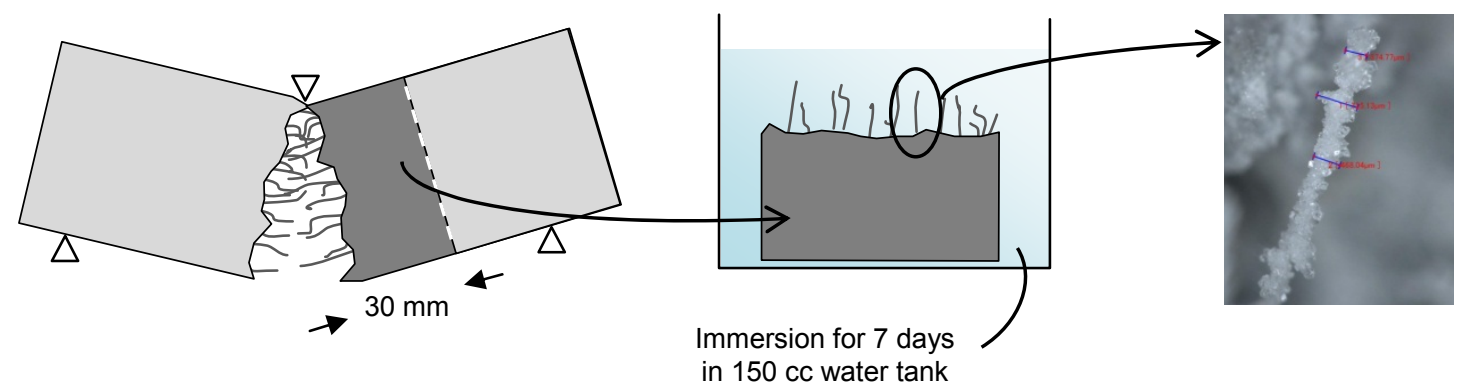

(a) Three-point bending test to split a specimen into 2 pieces (b) Immersion of a cut specimen in water (c) Measurement of the diameter of fibers with precipitated crystals

Fig. 2 Testing procedure of experiment $A$.

$30 \mathrm{~mm}$ height, and the broken surface was observed under microscope. Next, it was immersed in a water tank of $150 \mathrm{cc}$ volume. Figure 2 shows the schematic diagram of the testing procedure. Following immersion of the specimens in curing water for 7 days, fibers exposed from the broken surface were observed and the diameter of each fiber was measured using a digital microscope. Figure 2 (c) shows a sample of fiber covered by the precipitated crystals. The precipitation thickness was calculated from the difference between the measured whole diameter and the original diameter of the fiber. Here, the catalogue data was used as the original diameter because minimal scatter was confirmed by microscopic observation. Measurements were carried out over 15 points for all fibers projecting from the broken surface, and the thickness was calculated as the mean value.

\subsection{Experiment B (evaluation of self-healing capability of FRCCs containing different syn- thetic fibers)}

In experiment $\mathrm{B}$, self-healing capability was confirmed from morphological change of chemical products precipitated around cracks obtained by microscopic observation and from the coefficient of water permeability obtained by water permeability test. Figure 3 shows a specimen and the apparatus employed for the uniaxial tension test. The geometry of the specimen is a plate measuring $85 \mathrm{~mm} \times 85 \mathrm{~mm} \times 30 \mathrm{~mm}$ fixed in place with 4 screw bars (M6) with an anchor nut at the tip (Homma
2009). The same materials and mix proportion as those used in experiment A (Tables 1, 2 and 3) were employed. In the first curing step, the specimens were settled in a

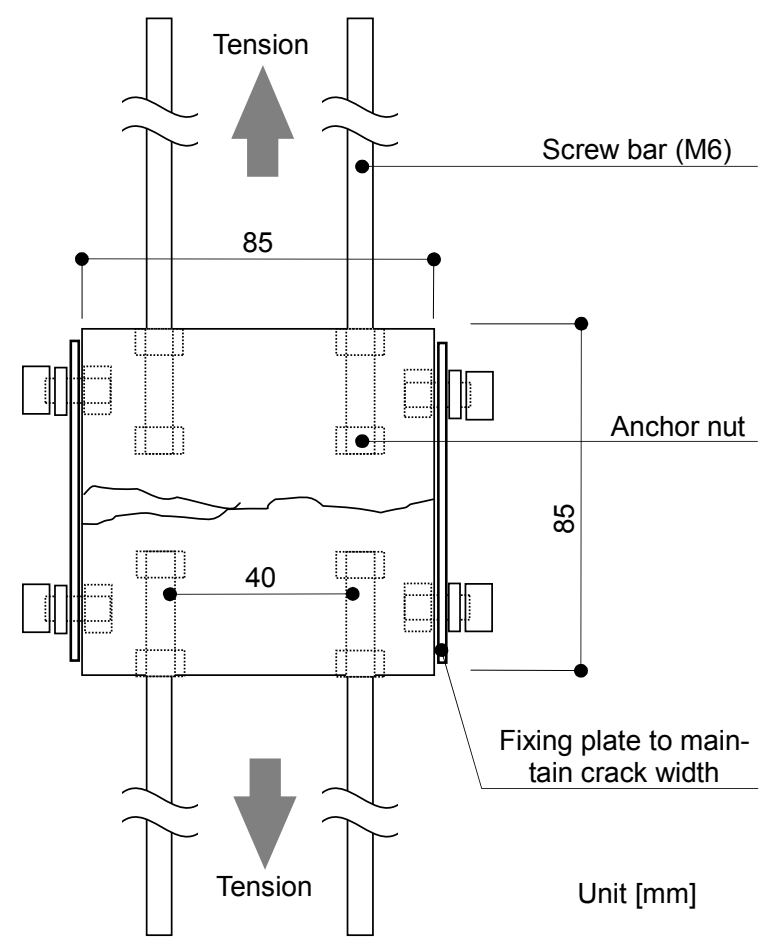

Fig. 3 Apparatus and specimen of experiment B. 


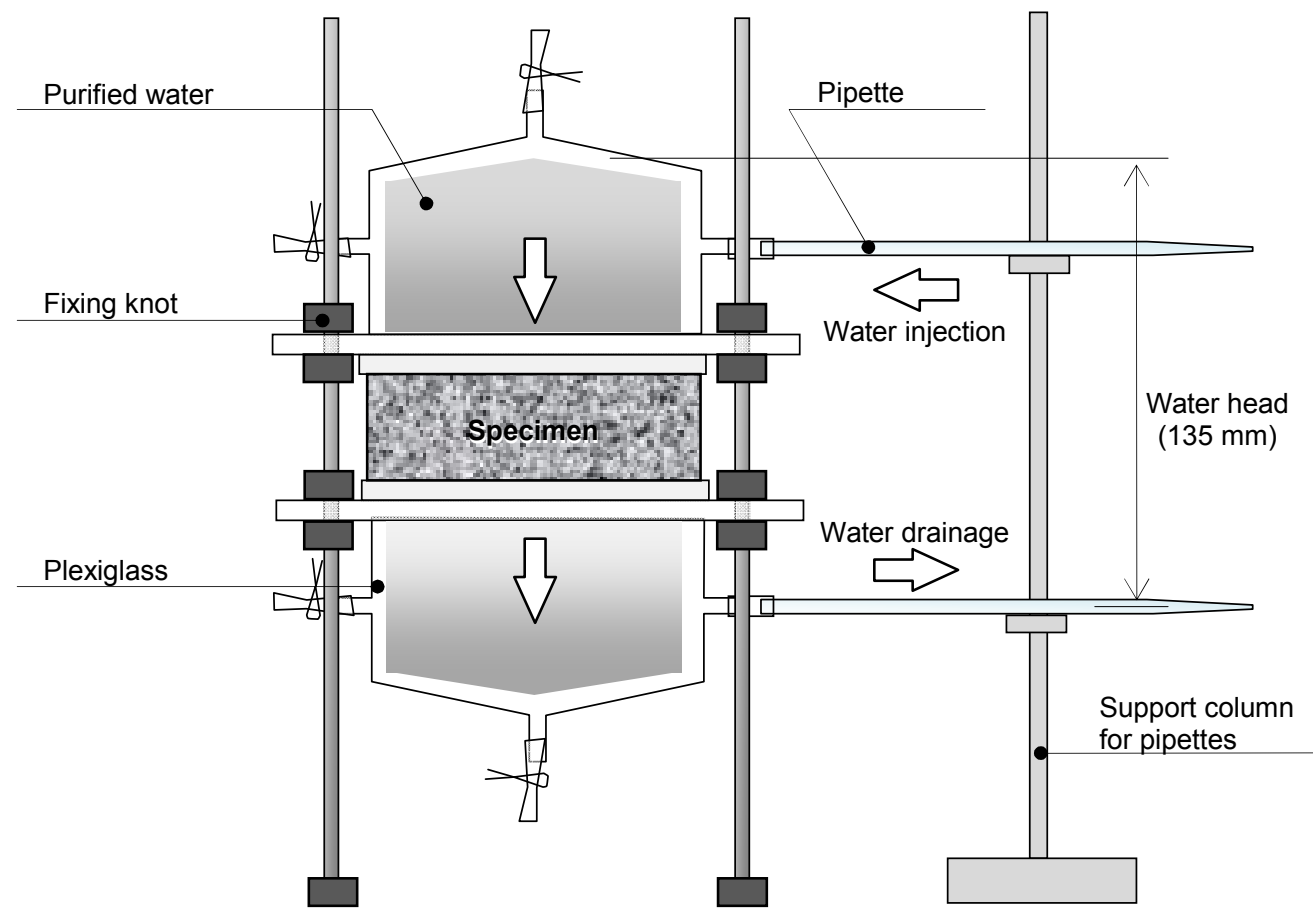

Fig. 4 Apparatus for water permeability test (Homma et al. 2009 and Kishimoto et al. 2007).

Table 4 Maximum crack width evaluated by means of microscopic observation $(\mu \mathrm{m})$.

\begin{tabular}{|c|c|c|c|c|c|c|}
\hline \multirow{2}{*}{$\begin{array}{c}\text { Speci- } \\
\text { men } \\
\text { No. }\end{array}$} & \multicolumn{7}{|c|}{ Series } \\
\cline { 2 - 7 } No. 1 & 240 & 301 & 350 & 287 & 423 & 225 \\
\hline No. 2 & 248 & 176 & 361 & 399 & 327 & 185 \\
\hline No. 3 & 250 & 195 & 367 & 339 & - & 106 \\
\hline
\end{tabular}

water tank of $20^{\circ} \mathrm{C}$ temperature and $50 \mathrm{~cm}$ water depth for 6 days after demolding. After the first curing step, the specimens were subjected to a uniaxial tension test using 4 embedded screw bars to generate a crack approximately $300 \mu \mathrm{m}$ wide. Metal plates were settled through the installed nuts on both sides of the specimen to maintain this crack width (Fig. 3). The maximum crack width of each specimen that was evaluated by means of microscopic observation is shown in Table 4. Except for the crack width, the three specimens were used under identical conditions. In the case of multiple cracking, the widest crack width of the main crack was measured as the maximum crack width, because the maximum crack width could be considered as a more sensitive parameter than average crack width for FRCC specimens featuring multiple cracks in order to evaluate the coefficient of water permeability (Nishiwaki 2004).

The cracked specimens were subjected to a water permeability test. Figure 4 shows the apparatus of this permeability test. This testing apparatus was developed from the method used by Kishimoto (2007) and Homma (2009). The coefficient of water permeability was calculated from the volume of permeating water through the specimen. After the test, as the second curing step, the specimens were immersed under the same conditions as the first curing step. During this second curing step, the water permeability test was carried out again after 3,14 and 28 days. At the same time, the surface around the crack was observed under digital microscope. After curing for 28 days (at the end of the second curing step), the specimens were subjected to the uniaxial tension test again, and the fractured surface of the specimens was observed under digital microscope to obtain information on the precipitation area and of the geometry of the crack surface.

\section{Results and discussion}

\subsection{Experiment $A$}

Figure 5 shows the average thickness of precipitated crystals of calcium carbonate on different types of fibers. According to this figure, the smallest precipitation was observed in the PP series. The precipitation thickness increased in the order of the POM, EVOH, PVA-I and PVA-II series. This tendency corresponds with the strength of polarity that was estimated from the chemical formula shown in Fig. 1. The polarity of the synthetic composite, e.g. hydroxide ions, suggests that the positively charged calcium ions are attracted by a strong electric field (Manoli, 2002). Therefore, a polarized fiber tends to attract $\mathrm{Ca}^{2+}$ ions and precipitate calcium carbonate. While the type of fibers used in the PVA-I and PVA-II series was the same but the diameters of the fibers differed between the two series, the fibers showed almost the same quantity of precipitated chemical products. Thus, it was revealed that the diameter of the fibers 


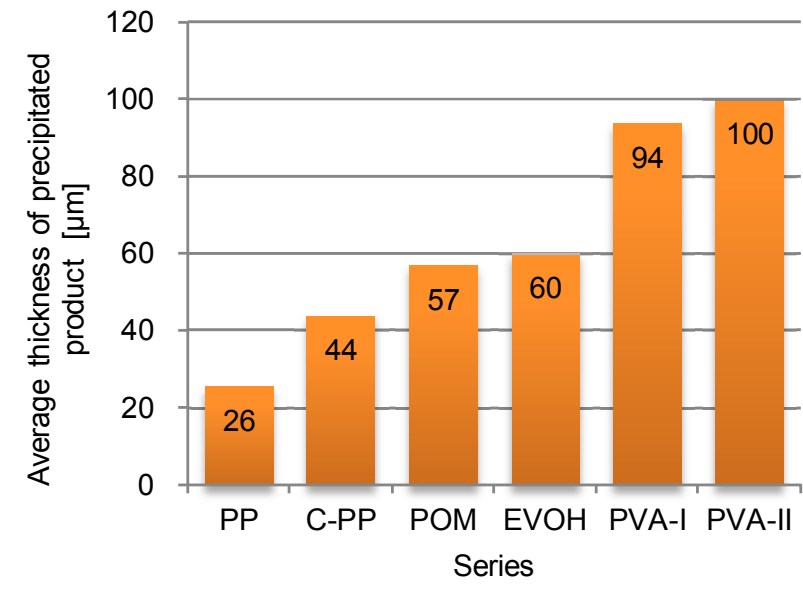

Fig. 5 Average thickness of precipitated chemical products.

has a slight influence on precipitation. Therefore, it is expected that thinner fibers with the same volume content can produce a larger number of cores on fibers bridging the crack than thicker ones, and that they can

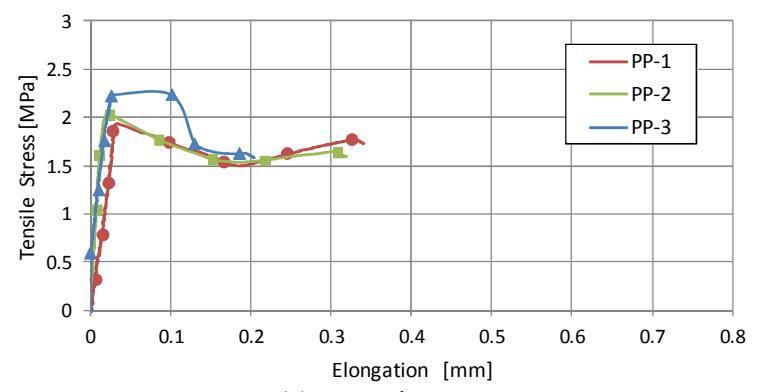

(a) PP series

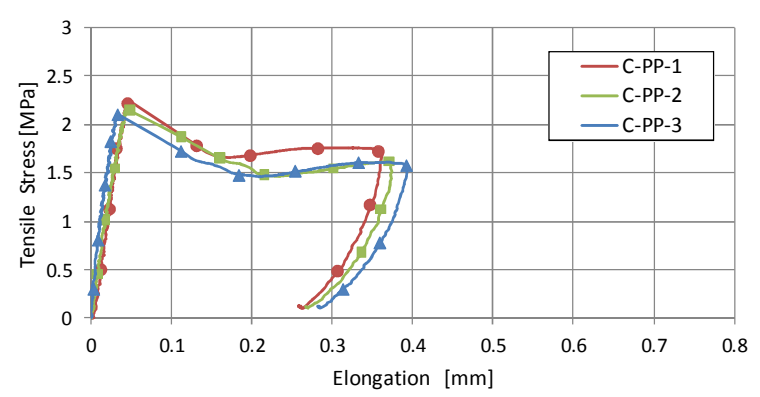

(c) C-PP series

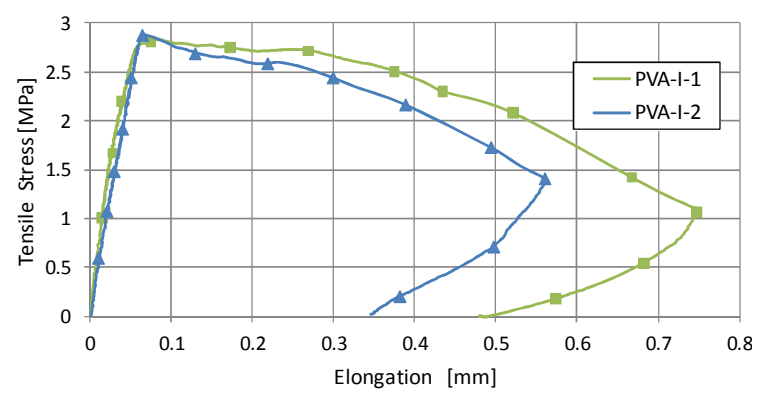

(e) PVA-I series supply a larger total quantity of self-healing precipitation in the crack. However, the tendency was different in the case of the C-PP and PP series, where a larger quantity of precipitation was measured in the former series than in the latter series. It was confirmed that fibers whose section is more complex and has a longer perimeter can provide a larger quantity of self-healing products than fibers whose section is of simple geometry.

\subsection{Experiment B \\ 4.2.1 Uniaxial tension test}

Figure 6 shows the relationship between tensile stress and elongation under the uni-axial tension test carried out for crack generation at the age of 7 days. According to this figure, the tensile strength of all the series was 2 to 3 $\mathrm{N} / \mathrm{mm}^{2}$, and ductility depends on the type of fiber. The PP, C-PP, PVA-I and PVA-II series showed ductile behavior even under uniaxial tensile stress. After the tension test, the residual crack width could be controlled to be approximately $300 \mu \mathrm{m}$. However, the POM and EVOH series showed brittle behavior and the specimens were almost instantaneously split into two parts just after the

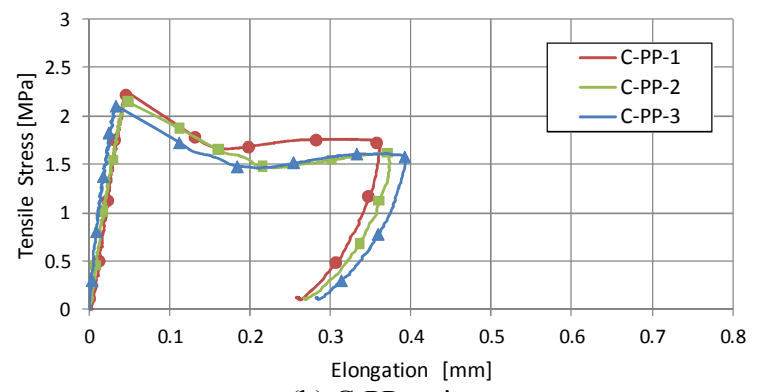

(b) C-PP series

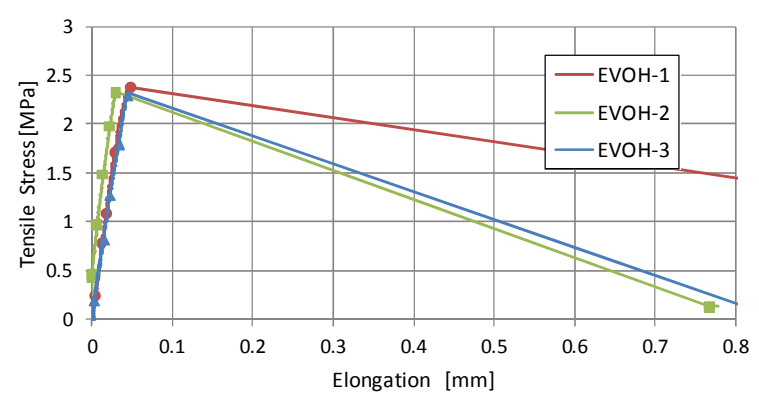

(d) EVOH series

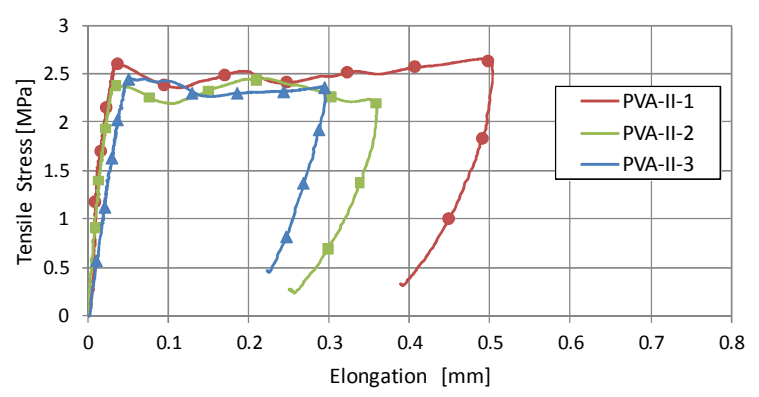

(f) PVA-II series

Fig. 6 Relationship between tensile stress and elongation in first loading test to generate a crack. 
peak load. The brittle behavior was due to the slight bond strength between the fibers and cement matrix, i.e. the bridging effect was not sufficient to obtain a ductile behavior. Therefore the unloaded specimens were set in the condition of a crack width of approximately $300 \mu \mathrm{m}$ using metal plates with embedded nuts and bolts.

\subsubsection{Microscopic observation}

Figure 7 shows the surface of each specimen including the maximum crack width obtained by microscopic observation. Just after the loading test (for crack generation), the edges of the crack could be clearly recognized. However, after water curing, self-healing products ap- peared and specimens containing different types of fibers showed different patterns of crack filling product precipitation. Concerning the PP series, crystallization products could be found only around the bridging fibers and they filled up only a small part of the crack even in the case of 14-day curing. In the other series, the crack of all the specimens was filled with precipitated crystals after 14-day curing. In both of the PVA series, remarkable self-healing was observed even after only 3 days of curing. These tendencies agree with the results of experiment $\mathrm{A}$ as discussed in section 4.1. In the case of ordinary plain concrete, the maximum crack width that can be filled by natural self-healing phenomena is under

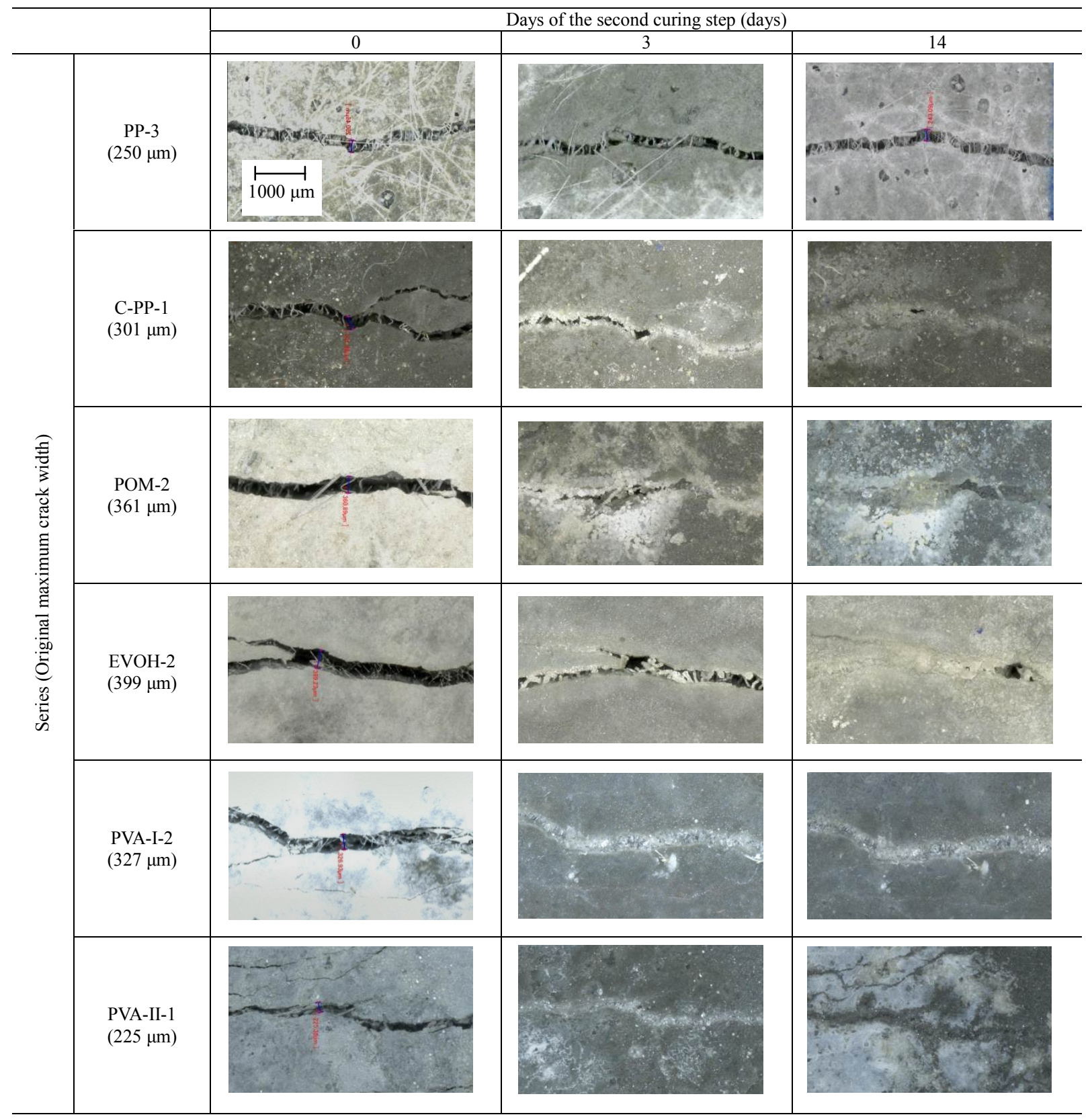

Fig. 7 Microscopic observation of precipitated products around crack. 
$0.1 \mathrm{~mm}$ (Edvardsen 1999). That is to say, FRCCs with synthetic fibers have greater self-healing capability than ordinary concrete.

\subsubsection{Water permeability test}

Figure 8 shows the relationship between curing days in the second curing step and the coefficient of water permeability $(k)$. The $k$ value was calculated by equation (4).

$$
k=\frac{Q \cdot l}{t \cdot A \cdot h}
$$

where $Q=$ quantity of water flow $\left[\mathrm{m}^{3}\right], l=$ thickness of the specimen $\left[\mathrm{m}^{2}\right], t=$ time [sec], $A=$ surface area of the plate specimen $\left[\mathrm{m}^{2}\right]$ and $h=$ pressure head $[\mathrm{m}]$ (here, $0.135 \mathrm{~m})$.

In Fig. 8, the $k$ value of the specimen without any crack is shown by dotted lines. Figure 9 (a) shows the normalized coefficient of water permeability in the case of the PP, C-PP, PVA-I and PVA-II series, which showed relatively ductile behavior under uniaxial tensile stress. The normalized coefficient of water permeability was estimated as the ratio of the value of $k$ in each curing day

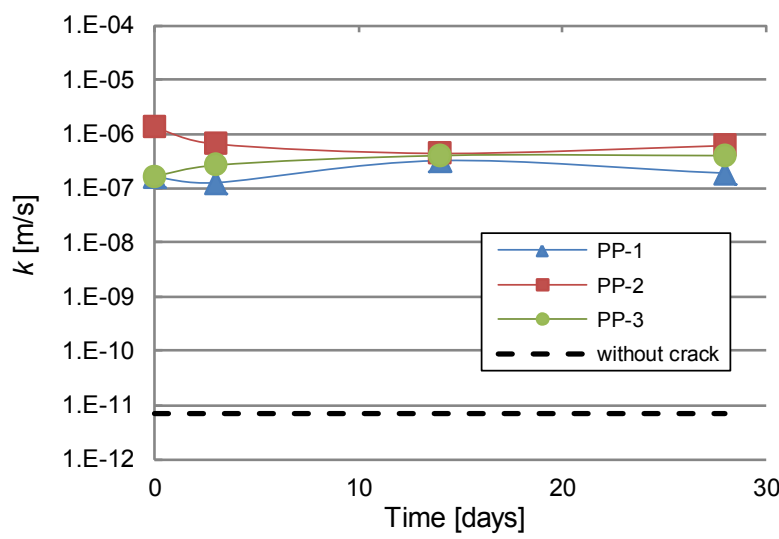

(a) PP series

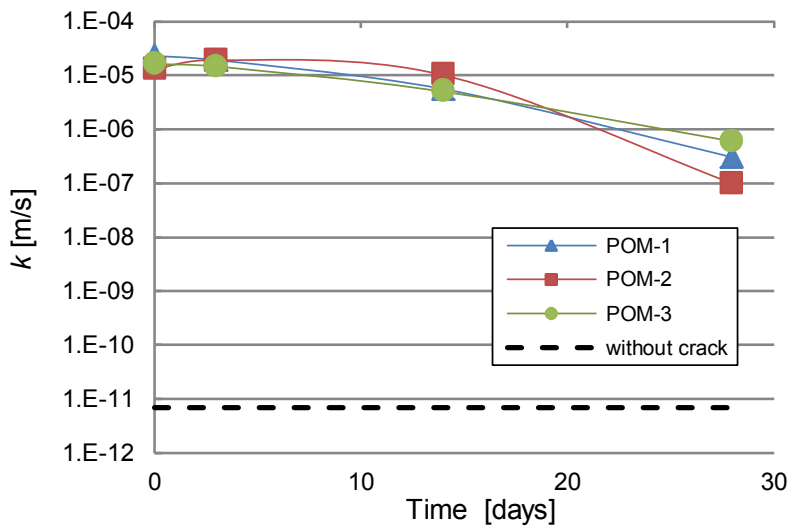

(c) POM series

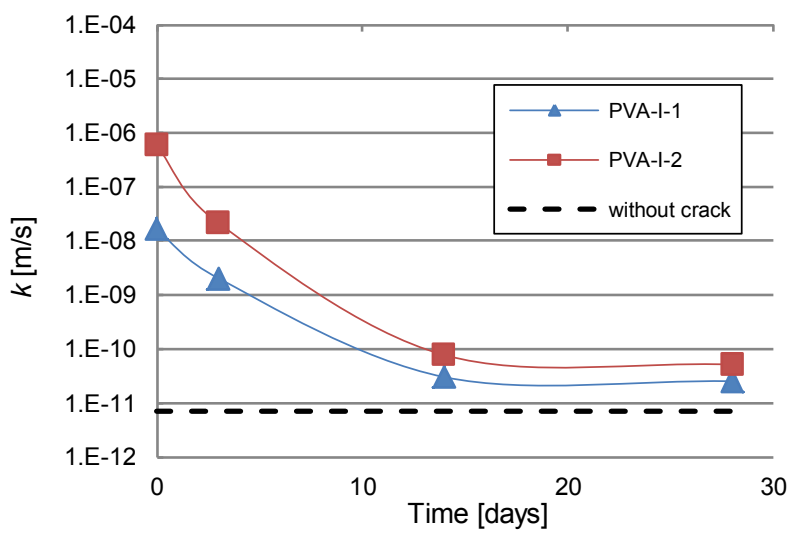

(e) PVA-I series

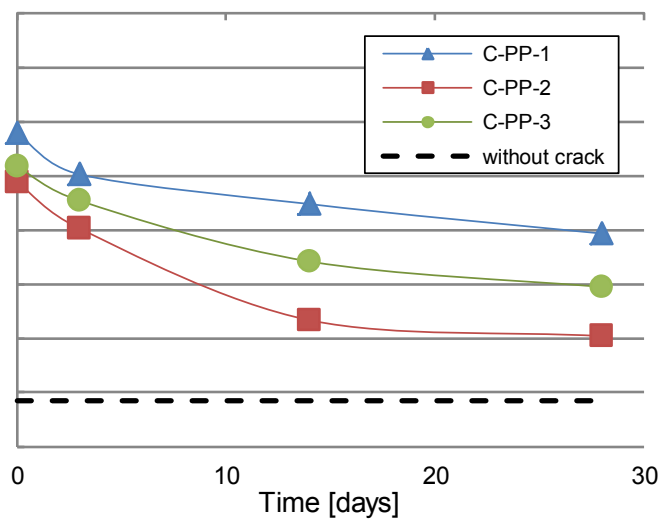

(b) C-PP series

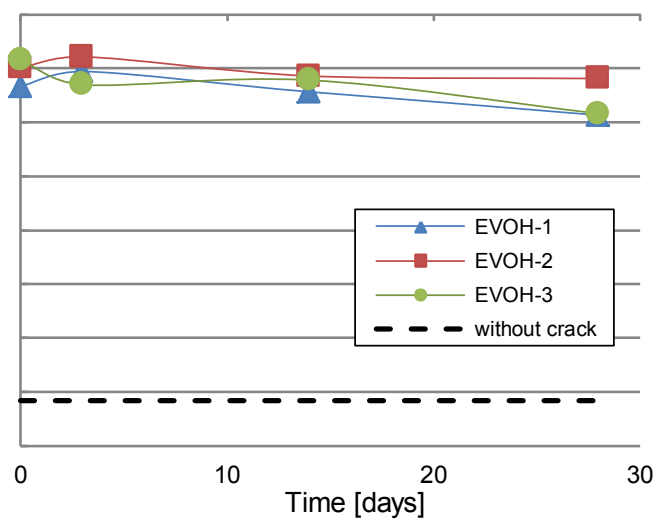

(d) EVOH series

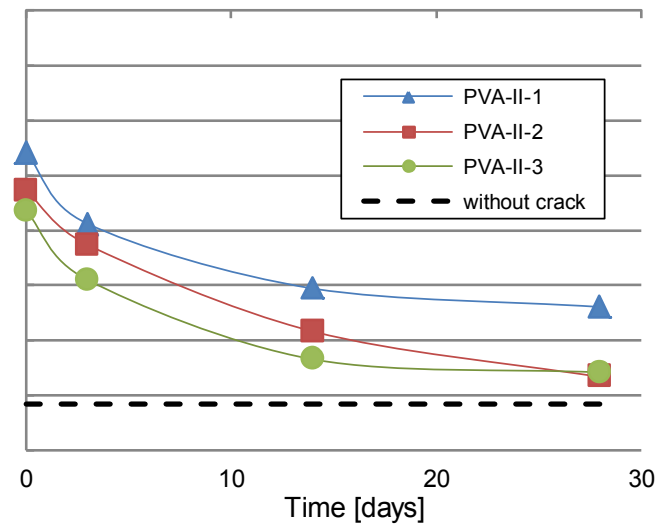

(f) PVA-II series

Fig. 8 Relationship between time and coefficient of water permeability. 


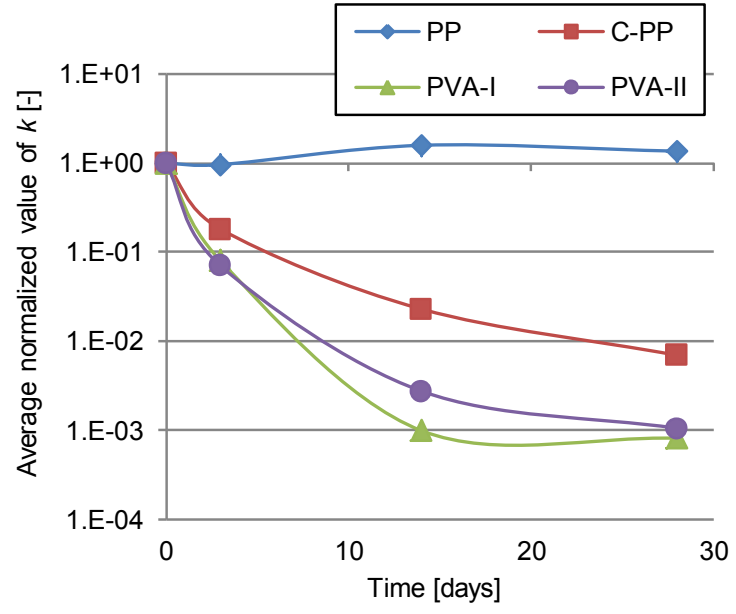

(a) PPs and PVAs series

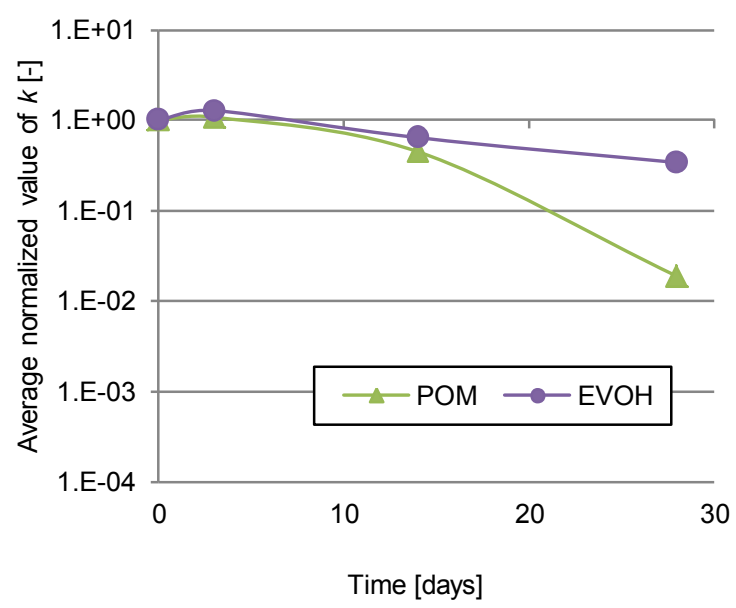

(b) POM and EVOH series

Fig. 9 Relationship between time and average normalized coefficient of water permeability.

to the $k$ value just after crack induction. As a result of self-healing phenomena, that is, filling of the crack with precipitated products, water permeability was reduced and water tightness was recovered. In the case of the PP series, however, the reduction of the coefficient of water permeability was not confirmed. By contrast, the C-PP specimen recovered its water tightness during the second curing step. In the cases of the PVA-I and PVA-II series, the coefficient of water permeability was remarkably reduced up to approximately 0.1 to $10 \times 10^{-11}(\mathrm{~m} / \mathrm{s})$, which approximates that of specimens without any cracks (approximately $0.7 \times 10^{-11} \mathrm{~m} / \mathrm{s}$ ). That is to say, taking the self-healing capability into account, FRCC using PVA fibers can allow a crack width of at least 0.3 $\mathrm{mm}$, which is much larger than the $0.1 \mathrm{~mm}$ width allowed in the case of ordinary concrete. Moreover, the PVA-I series reduced the coefficient of water permeability more effectively than the PVA-II series. This is due to the increase in the number of bridging fibers across the crack because PVA-I is much thinner than PVA-II. The employed type of synthetic fiber obviously affected the water tightness recovery ratio result.

Figures 8 (c) and (d) show the relationship between the number of days of the second curing and the coefficient of water permeability of the POM and EVOH series. Figure 9 (b) shows the relationship between time and the average normalized coefficient in the case of the POM and EVOH series, which showed brittle behavior in the tension test (see Figs 6 (c) and (d)). In both of these series, the coefficient of water permeability was not reduced by the self-healing phenomena, though self-healing products were observed under microscope as discussed in section 4.2.2. That is to say, self-healing products around the crack of the specimen cannot guarantee the recovery of water tightness. Since these series showed brittle behavior in the tension test, we considered that not only the chemical properties of the employed fibers but also the geometric properties of the crack surface affected the capability of self-healing. In the following section, the geometry of the crack surface was observed as one of the aspects of the FRCC specimen related to the mechanical properties.

\subsubsection{Observation of the fractured surface of the specimen}

After 28 days, that is the end of the second curing step, a uniaxial tension test was carried out again and the specimens were completely separated. Figure 10 shows detailed views of crack surfaces. Self-healing products remaining on the surface were observed, because the crack generated by the first loading was grown again in the same place by the second loading. The hexagonal shapes in the section are the marks left by the anchor nuts placed at the tip of the screw bars (Fig. 3). In almost all the specimens, the generated crack was located in the section including the anchor nuts. Self-healing products were found around the marginal area in all the series. However, self-healing products were observed in the center area of the crack surface only in the PVAs series. That indicates that the PVA series is more effective in creating self-healing products, including inside the crack.

As aspects of cracking properties, roughness and tortuosity of the crack surface were observed under digital microscope. Figure 11 shows the observed area and measured lines, which correspond to the data depicted in Fig. 13. The microscope employed in this study (VHX-1000, Keyence Corporation) has a function to automatically measure the depth of the surface three dimensionally. The depth is calculated based on the locations that are in (and out of) focus in obtained photographs (Nayar 1994). Figure 12 shows an example of the obtained contour map of a crack surface. From this figure, the surface area was automatically calculated by the digital microscope. Figure 13 shows the roughness of the crack surface measured along the five lines in Fig. 11. The PP and PVA-I series, which showed ductile behavior in the tension test, had rougher and more complex surfaces than the POM and EVOH series, which showed brittle behavior in the tension test. Moreover, Fig. 14 


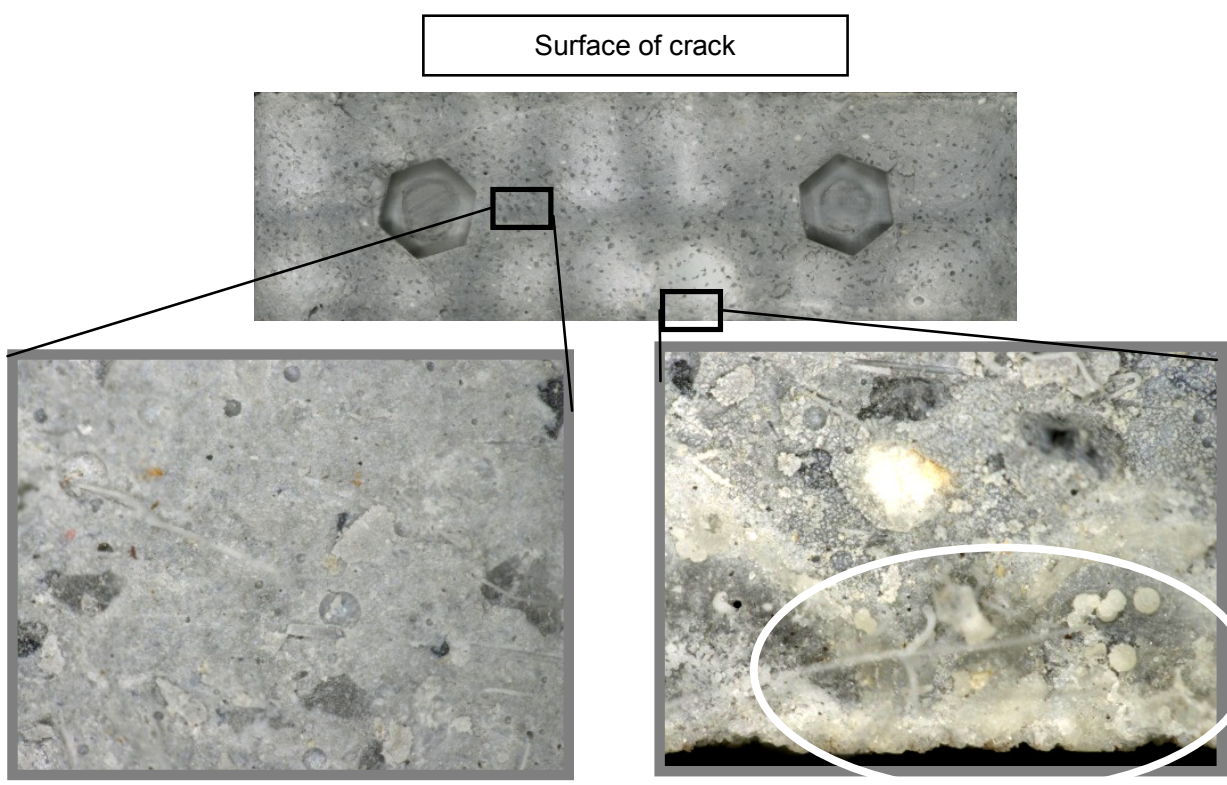

Center area of crack surface

Marginal area of crack surface

(a) POM series

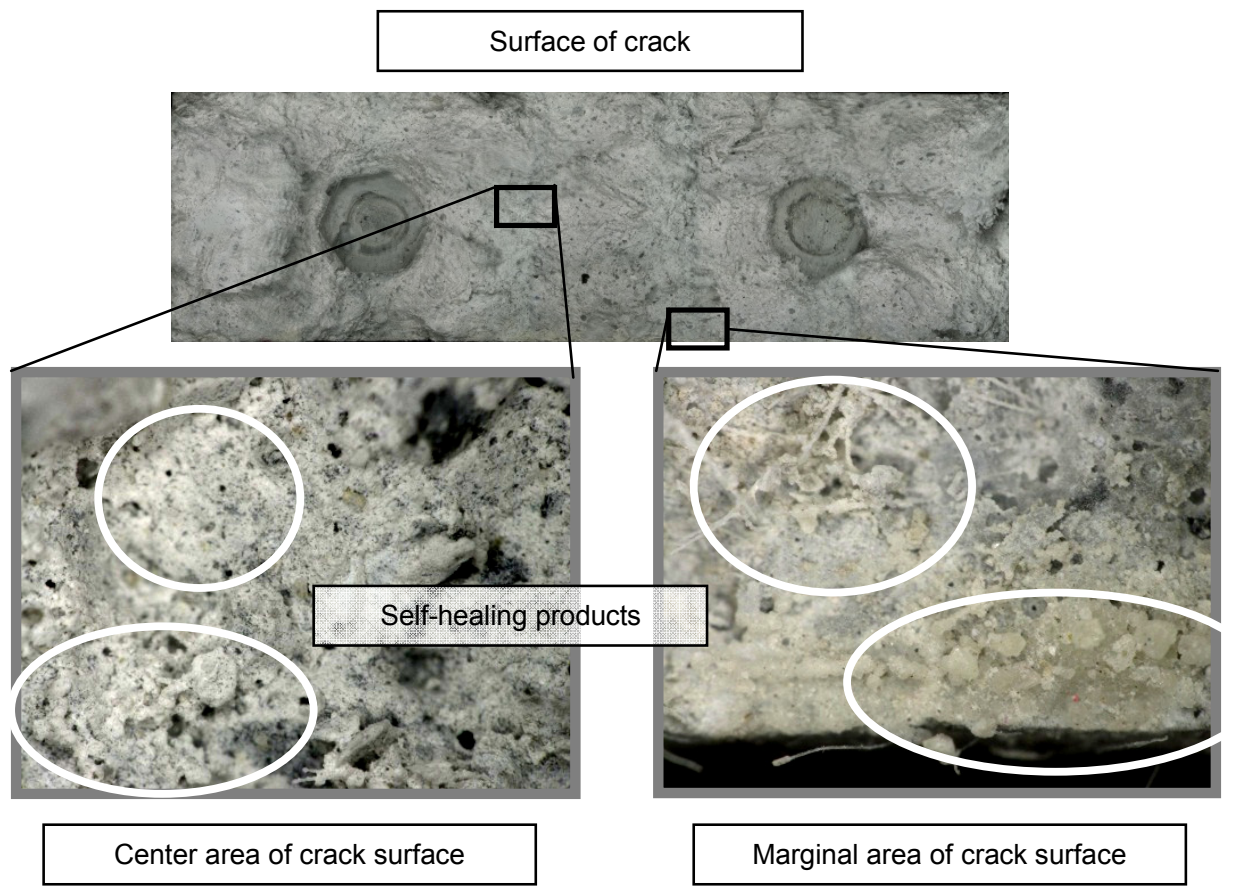

(b) PVA-I series

Fig. 10 Self-healing products on surface of crack.

shows the ratio of the crack surface area to the reference base area $\left(25 \times 35=875 \mathrm{~mm}^{2}\right)$. This figure clearly shows that the brittle series (POM and EVOH) had a smaller crack surface area and simpler surface geometry than the others series. On the other hand, the specimens of the ductile series (PPs and PVAs) had a complex and rougher surface, and the PVAs series in particular had the most complex crack surface geometry.
The water-flow rate through a crack in concrete is described as a function of the cube of the crack width per the following formula (Tsukamoto et al, 1991):

$$
q=\alpha \frac{g I \ell w^{3}}{12 v}
$$

where $q=$ water flow in concrete cracks $\left[\mathrm{m}^{3} / \mathrm{s}\right], \alpha=\mathrm{em}$ - 


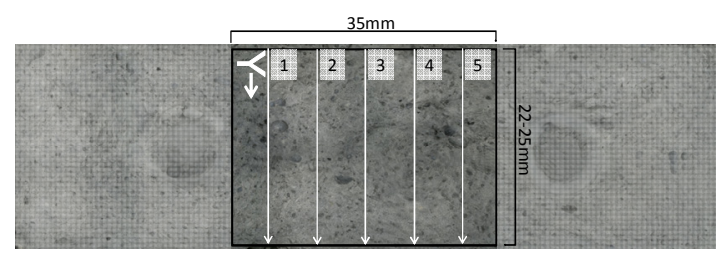

Fig. 11 Measured lines for roughness of crack surface.

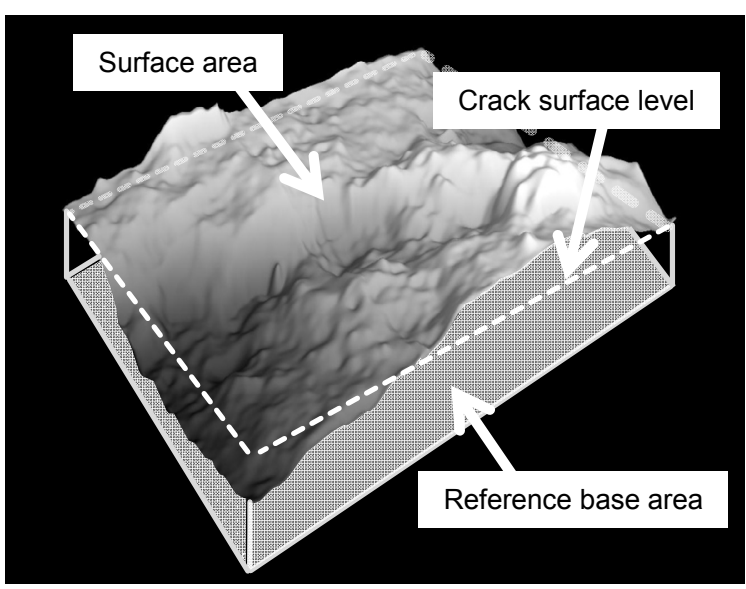

Fig. 12 Calculated surface area and reference base area.

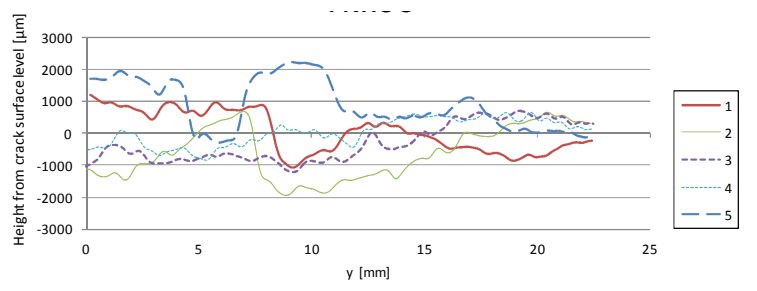

(a) PP series

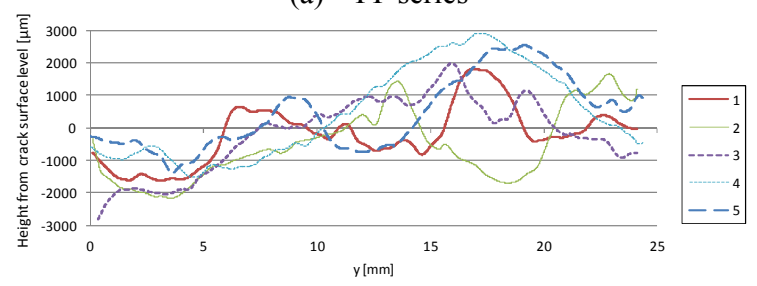

(b) PVA-I series

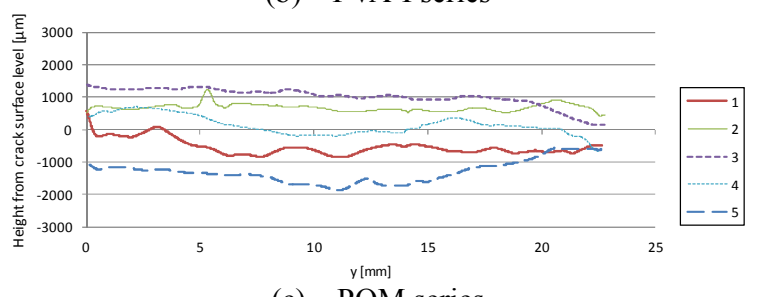

(c) POM series

Fig. 13 Roughness of crack surface.

pirical parameter [-], $g=$ gravity acceleration $\left[\mathrm{m} / \mathrm{s}^{2}\right], I=$ pressure gradient $(h / L), h=$ height of fluid column on the inlet side [m], $L=$ crack length in the flow direction [m], $\ell=$ crack length at a right angle to the flow direction $[\mathrm{m}], w=$ crack width $[\mathrm{m}]$, and $v=$ kinematic viscosity $\left[\mathrm{m} / \mathrm{s}^{2}\right]$.

Here, we can recognize $\alpha$ as a coefficient related to the

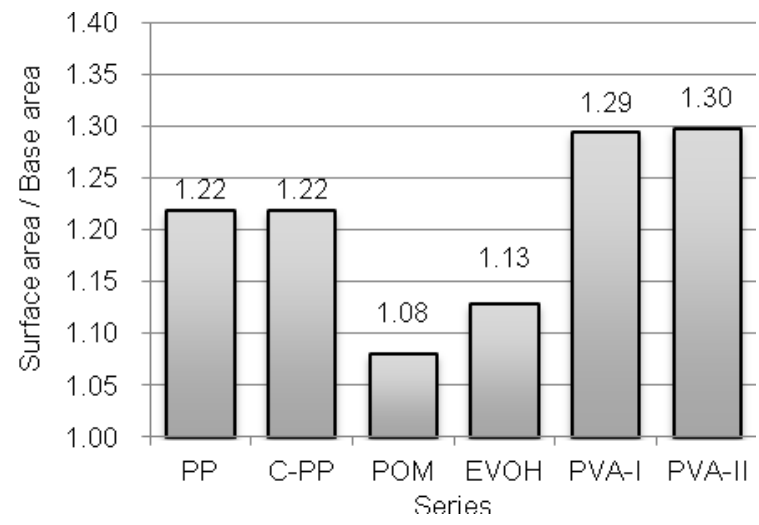

Fig. 14 Ratio of crack surface to reference base area.

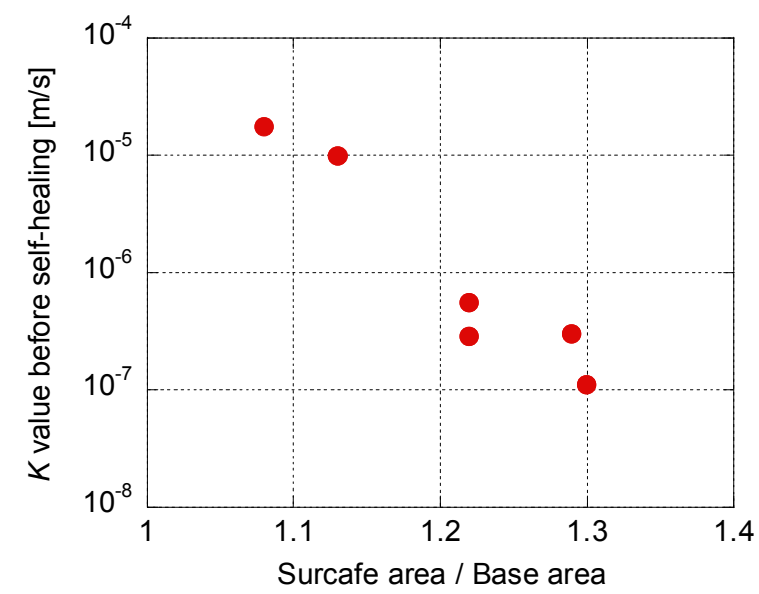

Fig. 15 Relationship between ratio of crack surface to reference base area and coefficient of water permeability before self-healing.

geometry of the crack surface differing from a smooth plate, e.g. roughness of crack surface, crack branching, and accumulation of microcracks around the main crack. Thus, $\alpha$ is related to the ratio shown in Fig. 14. In this study, the crack width of the specimens was adjusted to approximately $300 \mu \mathrm{m}$. Before the self-healing process, the coefficients of water permeability $k$ and $\alpha$ are related to each other (Fig. 15). Homma et al. (2009) demonstrated that more complex crack surfaces have greater capability of self-healing. Here, the ductile series with larger $\alpha$ (PVAs) reduced the water permeability more effectively than the brittle series (EVOH and POM). The specimens of the ductile series tended to have multiple cracks and crack branches while the specimens of the brittle series were separated into two pieces with a single crack. Such complex and discontinuous cracks have a fracture process zone at the tip, where self-healing products can be easily deposited because of their rather narrow crack width. However, the PPs series, which had mediate level $\alpha$, had lower capability of self-healing because of lack of polarity of reinforcing fibers. Namely, both of the ductility and chemical properties were required in order to enhance the capability of self-healing 


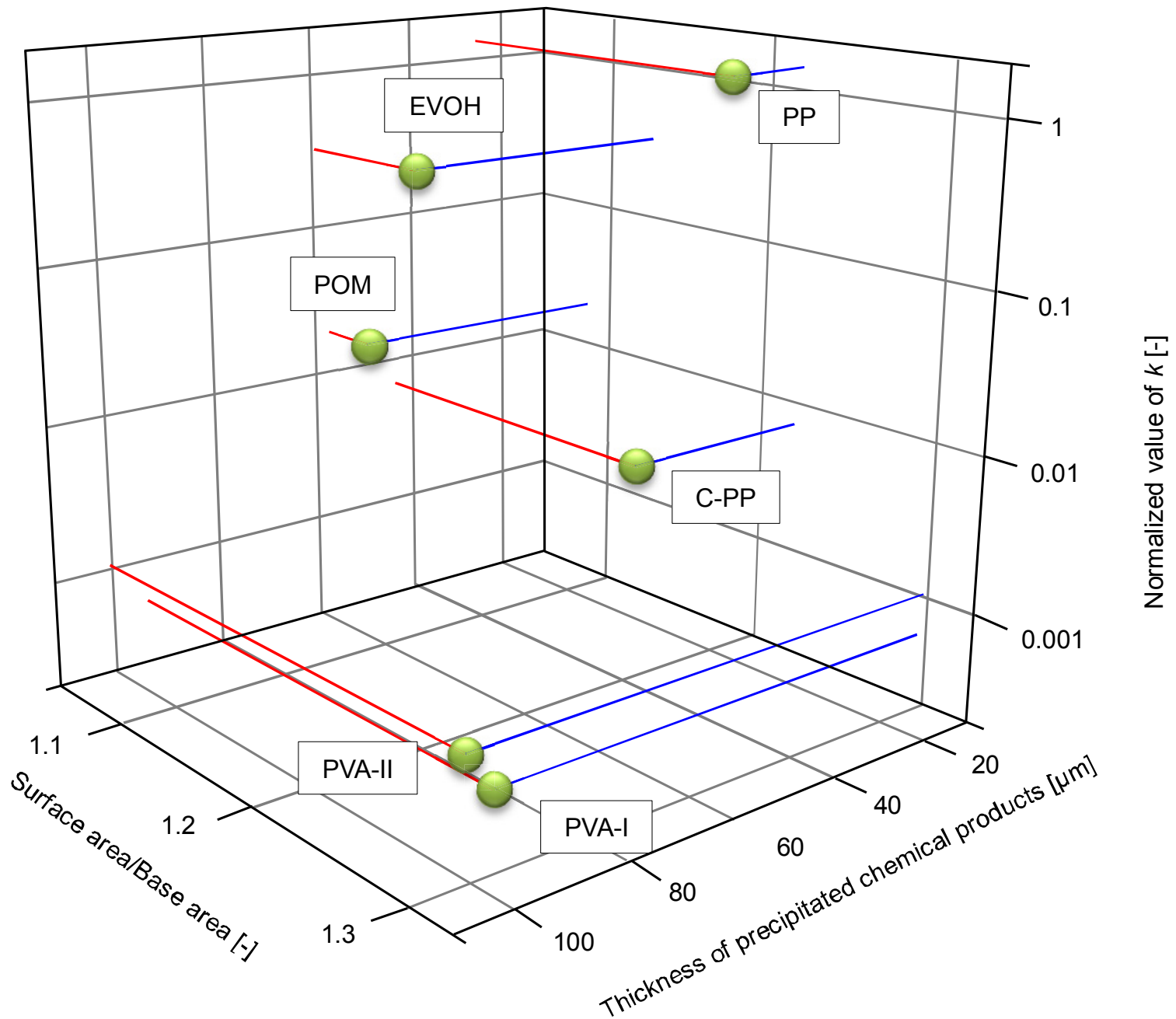

Fig. 16 Relationship between normalized coefficient of water permeability, thickness of self-healing precipitation and ratio of crack surface to reference base area.

of FRCC. These results are summarized in Fig. 16, which shows the relationship between the capability of self-healing (the normalized coefficient of water permeability), the polarity of the employed fibers (the thickness of self-healing precipitation), and the complexity of the geometry of the crack surface (the ratio of the crack surface to the reference base area).

Based on the findings of the present study, it is suggested that selecting the optimal type of fiber most effective for self-healing of FRCC should be done from the viewpoint of not only the potential of chemical precipitation but also the cracking properties of the whole FRCC material.

\section{Concluding remarks}

This paper presents the results of experimental studies on the self-healing capability of FRCC using different types of synthetic fibers. Based on the results, the following conclusions can be drawn:

(1) FRCC using synthetic fibers have a great self-healing capability, because synthetic fibers not only control crack width but also serve as a core of the chemical precipitation site. In particular, synthetic fibers with high polarity can promote effective precipitation of self-healing products.

(2) The PVAs series, which have the highest polarity, can recover water tightness by self-healing phenomena for crack widths up to $0.3 \mathrm{~mm}$.

(3) It is confirmed that not only the chemical properties of fibers but also the geometrical properties of the crack surface, i.e. roughness, complexity and continuity, affect the capability of self-healing for water tightness.

\section{Acknowledgement}

This research was partially supported by a Grant-in-Aid for Young Scientists (A) from the Ministry of Education, Culture, Sports, Science and Technology (\#23686078, 2011-2014).

\section{References}

Ahn, T. H. and Kishi, T., (2010). "Crack self-healing behavior of cementitious composites incorporating 
various mineral admixtures." Journal of Advanced Concrete Technology, 8(2), 171-186.

Edvardsen, C., (1999). "Water permeability and autogenous healing of cracks in concrete." $A C I$ Materials Journal, 96(96-M56), 448-454.

Homma, D., Mihashi, H. and Nishiwaki, T., (2009). "Self-healing capability of fibre reinforced cementitious composites." Journal of Advanced Concrete Technology, 7(2), 1-12.

Igarashi, S. Kunieda, M. and Nishiwaki, T. (2009). "Research activity of JCI technical committee TC-075B: Autogenous healing in cementitious materials." In: Proceedings of 4th International Conference on Construction Materials: Performance, Innovations and Structural Implications (Nagoya, Japan), 89-96.

Jonkers, H. M., Thijssen, A., Muyzer, G., Copuroglu, O. and Schlangen, E., (2010). "Application of bacteria as self-healing agent for the development of sustainable concrete.” Ecological Engineering, 36, 230-235.

Kan, L. L., Shi, H. S., Sakulich, A. R. and Li, V. C., (2010). "Self-healing characterization of engineered cementitious composite materials." ACI Materials Journal, 107(6), 617-624.

Kishimoto, Y. Hokoi, S., Harada, K. and Takada, S., (2007). "The effect of vertical distribution of water permeability on the modeled neutralization process in concrete walls." Journal of ASTM International, 4(1), Paper ID JAI100323.

Koda, M., Mihashi, H., Nishiwaki, T., Kikuta, T. and Kwon, S. M., (2011). "Self-healing capability of fiber reinforced cementitious composites." In: Proceedings of the 3rd International Symposium on Advances in Concrete through Science and Engineering (Hong Kong, China), RILEM, CD-ROM (abstract 119).

Li, V. C., Lim, Y. M. and Chan, Y.-W., (1998).
"Feasibility study of a passive smart self-healing cementitious composite." Composites- Part B, 29B, 819-827.

Manoli, F., Kanakis, J., Malkaj, E. and Dalas, E., (2002). "The effect of aminoacids on the crystal growth of calcium carbonate." Journal of Crystal Growth, 236, 363-370.

Mihashi, H., Ahmed, S. F. U. and Kobayakawa, A., (2011). "Corrosion of reinforcing steel in fiber reinforced cementitious composites." Journal of Advanced Concrete Technology, 9(2), 159-167.

Nayar, S. K. and Nakagawa, Y., (1994). "Shape from focus." IEEE Transactions on Pattern Analysis and Machine Intelligence, 16(8), 824-831.

Neville, A.vM., (1996). "Properties of concrete." 4th ed. Essex :Pearson Education Limited.

Nishiwaki, T., Leite, J. P. B. and Mihashi, H., (2004). "Enhancement in durability of concrete structures with use of high-performance fibre reinforced cementitious composites." In: Proceedings of the Fourth International Conference on Concrete under Severe Conditions: Environment \& Loading; CONSEC'04, 2, 1524-1531.

Qian, S., Zhou, J., De Rooij, M. R., Schlangen, E., Ye, G., and Van Breugel, K., (2009). "Self-healing behavior of strain hardening cementitious composites incorporating local waste materials." Cement \& Concrete Composites, 31, 613-621.

Reinhardt, H. W. and Joose, M., (2003). "Permeability and self-healing of cracked concrete as a function of temperature and crack width." Cement and Concrete Research, 33, 981-985

Tsukamoto, M. and Woerner, J. D., (1991). "Permeability of cracked fibre-reinforced concrete." Darmstadt Concrete, 6, 123-135. 
Invited paper

\title{
Robust Self-Healing Concrete for Sustainable Infrastructure
}

\author{
Victor C. $\mathrm{Li}^{1}$ and Emily Herbert ${ }^{2}$
}

\section{Abstract}

This article introduces the concept of self-healing concrete for sustainable infrastructure through reduction of maintenance and repair in the use phase. To realize this goal, self-healing must observe at least six robustness criteria - long shelf life, pervasive, quality, reliable, versatile, and repeatable. Five broad categories of self-healing approaches, namely chemical encapsulation, bacterial encapsulation, mineral admixtures, chemical in glass tubing, and intrinsic healing with self-controlled tight crack width, are evaluated against the robustness criteria. It is suggested that while significant progress has been made over the last decade in laboratory studies, important knowledge gaps must be filled in all categories of self-healing approaches to attain the goal of smart sustainable infrastructures that possess self-repair capability in the field.

\section{Introduction}

Many developed countries, including Germany, S. Korea, and the US, are experiencing unprecedented amounts of civil infrastructure deterioration, so much so that the annual outlay for repair and rehabilitation has outstripped the cost of new infrastructure construction. The annual economic impact associated with maintaining, repairing, or replacing deteriorating structures is estimated at \$18-21 billion in the U.S. alone (Vision 2020 2006). The American Society of Civil Engineers estimates that $\$ 2.2$ trillion is needed over the next five years for repair and retrofit; a cost of $\$ 2$ trillion has been estimated for Asia's infrastructure. To make matters worse, repairs of concrete structures are often shortlived. In the US, it is estimated that half of all field repairs fail and require re-repairs (Mather and Warner 2003). The concerns related to civil infrastructure deterioration are not limited to the economic cost of repair and rehabilitation, but extend to social and environmental costs. While there is little documentation and quantification of the social and environmental costs, it is generally agreed that repeated repairs of civil infrastructure over their service life is decidedly unsustainable.

Over the last decade, the concept of concrete infrastructure able to repair itself without human intervention has emerged as a possible cure for overcoming civil infrastructure deterioration. While the idea remains a novelty in practice, it has attracted a significant amount of attention in the research community. Many different approaches to functionalizing concrete to possess self-

${ }^{1}$ Professor, Department of Civil and Environmental Engineering, University of Michigan, Ann Arbor, MI 48109-2125 USA

E-mail:vcli@umich.edu

${ }^{2}$ Department of Civil and Environmental Engineering, University of Michigan, Ann Arbor, MI 48109-2125 USA healing ability have been investigated. Given that damage in concrete is dominated by cracks, much attention has been given to self-repair of cracks. In a few cases, field trials have been launched. These studies hold promise to the feasibility of future civil infrastructure smart enough to detect its own damage and undergo repair by itself. Thus self-healing concrete has significant implications in extending service life, and reducing economic, social and environmental costs of civil infrastructure. That is, self-healing concrete could be a major enabling technology towards sustainable civil infrastructure.

Figure 1 shows the number of publications related to self-healing concrete over the last decade. It paints a picture that we have entered into an intense phase of research on the development of self-healing concrete, and the pace of research will likely continue to accelerate. A survey of the literature indicates that this development is spread over the major continents, and is particularly intense in Europe and Japan.

There have been a number of review articles on selfhealing concrete [see, e.g. Wu et al. 2012, RILEM TC-

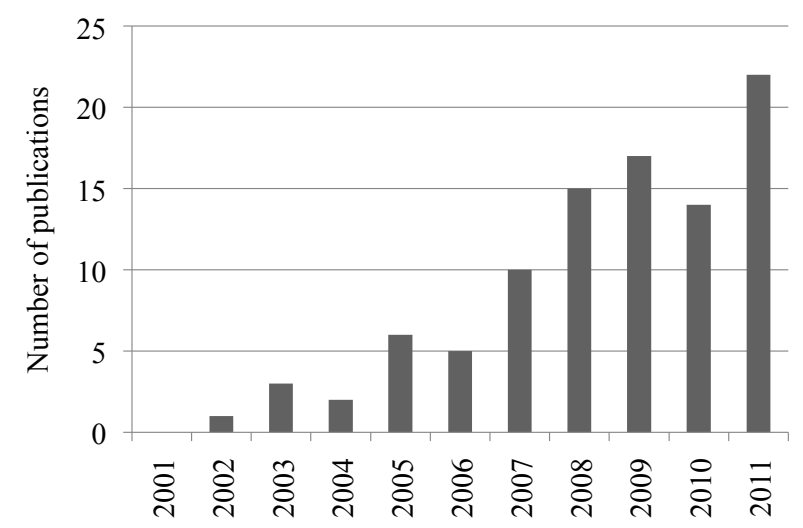

Fig. 1 Archived journal publications on self-healing concrete over the last decade (2001-2011) (Source: Google Scholar on-line) 


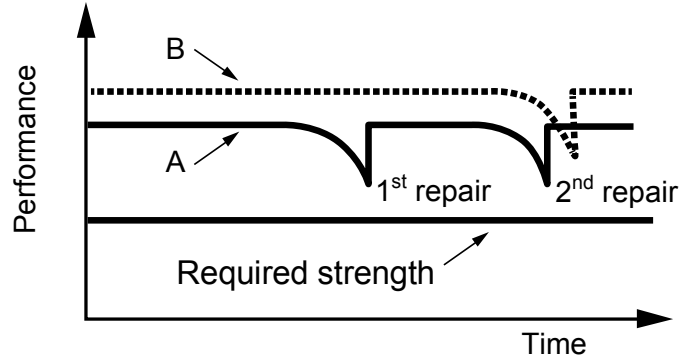

(a)

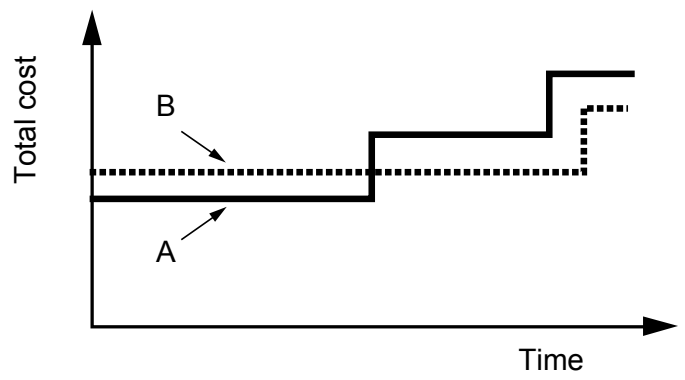

(b)

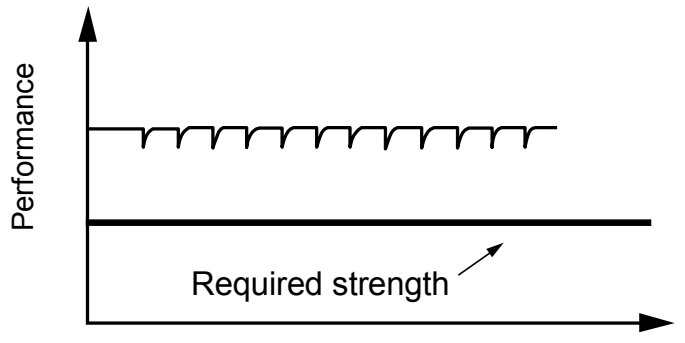

(c)

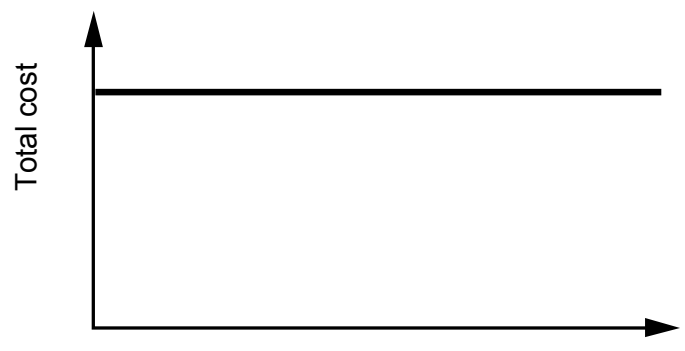

(d)

Fig. 2 (a) and (b): Conceptual life-cycle cost model emphasizing cost increase associated with repair events for conventional concrete structure; The dotted lines are for a higher performance higher cost concrete B compared with concrete $A ;(c)$ and (d): Corresponding life-cycle cost and performance when self-healing is included (van Breugel, 2007).

221-SHC STAR]. The purpose of the present article is not to repeat these reviews, but rather to propose the concept of robust self-healing - a set of robustness criteria against which several generic self-healing approaches can be evaluated. As such, the literatures referenced are not meant to be exhaustive of what has been published. The objective of such an evaluation is aimed at highlighting advantages and limitations of various self-healing approaches, to spur additional research to overcome current limitations, and to generally accelerate convergence of concrete technology developments that support the realization of smart sustainable infrastructure systems with self-repair functionality. The robustness criteria serve as a filter for self-healing approaches under study in laboratories and identify those that are likely effective in infrastructure damage selfrepair in the field.

\section{Impact of self-healing on infrastructure life cycle cost}

Some direct benefits of concrete self-healing include the reduction of the rate of deterioration, extension of service life, and reduction of repair frequency and cost over the life cycle of a concrete infrastructure. These direct benefits may be expected to lead to enhanced environmental sustainability since fewer repairs implies lower rate of material resource usage and reduction in energy consumption and pollutant emission in material production and transport, as well as that associated with traffic alterations in transportation infrastructure during repair/reconstruction events.
Van Breugel (2007) suggested a conceptual life cycle performance and cost model. The current practice of periods of gradual infrastructure deterioration punctuated by discrete repair events leads to increasingly high cumulative costs that may match or even exceed the initial construction cost (Fig. 2a,b). In contrast, infrastructure built with self-healing concrete may have higher initial cost, but the self-healing functionality maintains the quality of infrastructure with minimum or no additional cost accumulation over the life cycle, resulting in a life-cycle cost that could be competitive with that of current concrete infrastructure (Fig. 2c,d).

A simple quantitative life cycle cost model was proposed by Li et al. (2010) for reinforced concrete infrastructure subjected to a chloride environment. The service life and life cycle cost analysis framework is depicted in Fig. 3. Using a reinforced concrete bridge deck as illustration, the service life is assumed to be governed by the time to corrosion initiation $t_{i}$ of steel reinforcing bars as influenced by the rate of chloride diffusion through the cover concrete, and corrosion propagation $t_{p}$ as influenced by the spalling propensity of the concrete cover. Life-365 software (Life-365, 2012) was used to compute the service life and life cycle cost. In this model experimental data for chloride diffusion coefficient in cracked concrete serve as the input parameter in Life-365.

The presence of self-healing alters the crack pattern, reducing the crack width (lower right corner box in Fig. 3). Complete sealing is assumed to take place when concrete cracks are limited to below $30 \mu \mathrm{m}$, as has been experimentally demonstrated (Yang et al. 2009). This 


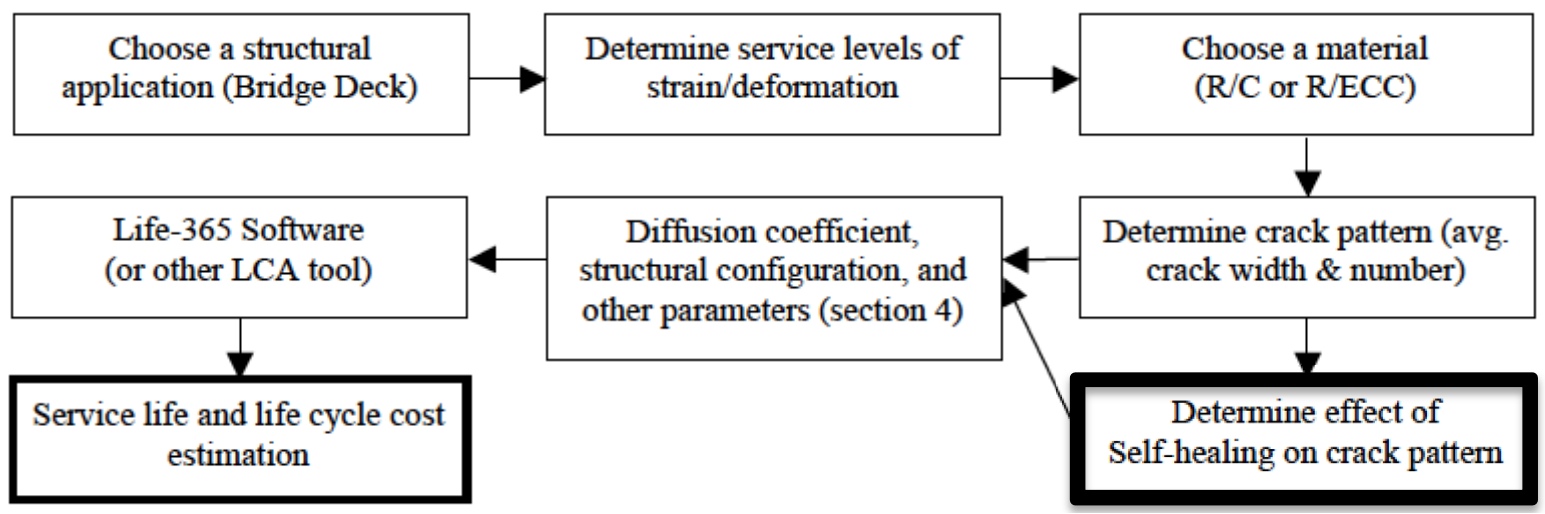

Fig. 3 Service life and life cycle cost estimation framework accounting for self-healing functionality in concrete (Li et al, 2010).

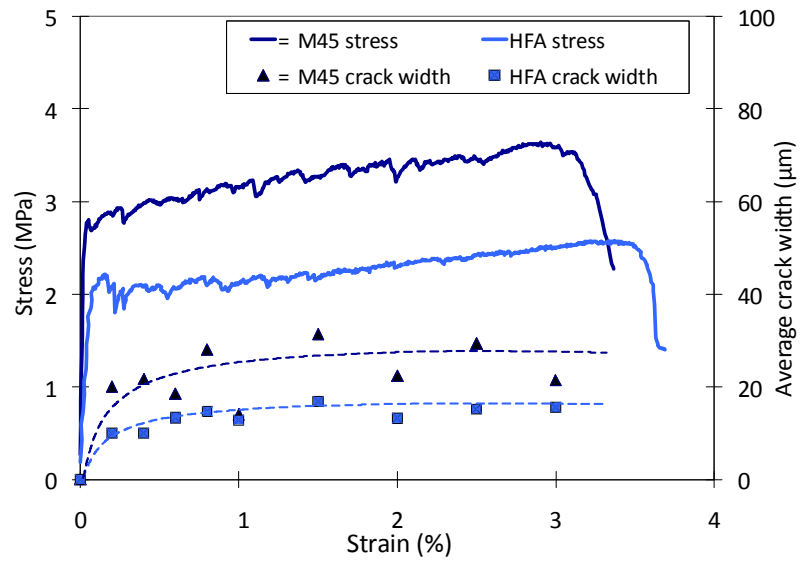

Fig. 4 Tensile Stress-Strain Curve and Crack width Development of Two ECCs. Tight crack width can be designed down to below $20 \mu \mathrm{m}$, even at tensile strain exceeding $2 \%$.

results in a lowering of the chloride diffusion coefficient that forms the input to Life-365 that computes the lengthened service life and reduction in life cycle cost.

The realization of tight crack width for self-healing was achieved in Engineered Cementitious Composite (ECC) (Li et al. 2002; Li 2003). ECC exhibits tensile strain hardening with strain capacity of several percent. During strain hardening, multiple microcracking takes place with crack width limited to below $60 \mu \mathrm{m}$. Typical tensile stress-strain curves and crack width development in ECC are shown in Fig. 4.

The measured effective diffusion coefficient $D_{e}$ for ECC was found to increase linearly (Sahmaran et al. 2007) with imposed tensile strain (Fig. 5a). For normal concrete, the measured effective diffusion coefficient was found to increase exponentially with tensile deformation. In terms of crack width, the diffusion coefficient was found to scale parabolically as shown in Fig. 5b. For ECC, the linear increase in $\mathrm{D}_{\mathrm{e}}$ was due to increase in crack number while the crack width remains essentially fixed even as deformation increases to a large value (Fig. 4).

For comparison with ECC with an imposed strain of $0.3 \%$ (concrete fractures at $0.01 \%$ ), a normal concrete

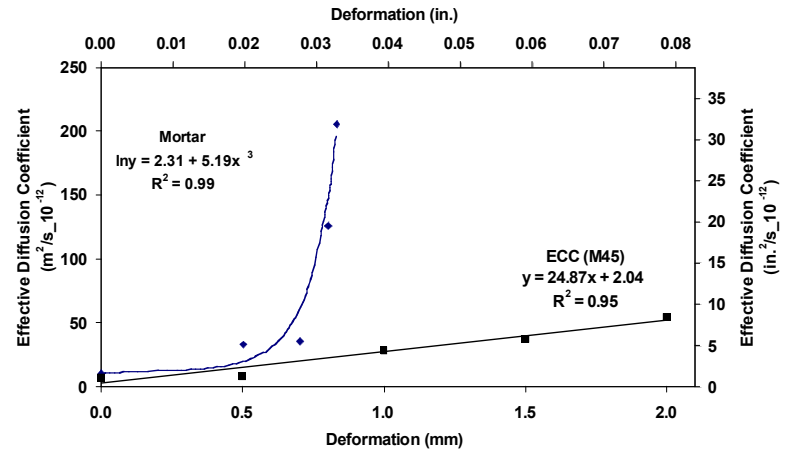

(a)

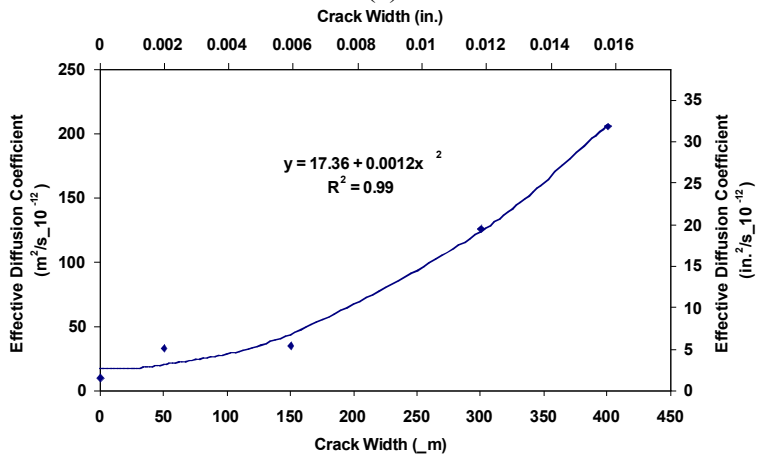

(b)

Fig. 5 Chloride Diffusion Coefficient (a) as a function of specimen flexural deformation in mortar and ECC and (b) as a function of crack width for mortar (after Sahmaran et al 2007). These experimental data are used to compute the service life and life cycle cost (Figure 3).

with crack width up to $400 \mu \mathrm{m}$ (allowable by AASHTO for exterior exposure, AASHTO 2004) was adopted in the study on service life and life cycle cost by Li et al. (2010). A high chloride exposure $(0.75 \%$ weight concentration on concrete surface) on a bridge deck located in Detroit, MI was specified. A corrosion inhibitor of 15 liter $/ \mathrm{m}^{3}$ was further assumed. (Other imposed deformation values and corrosion inhibitor values were also analyzed, but the main conclusions remained essentially the same.)

The computed service life is summarized in Fig. 6a. The presence of inhibitor increases the time to corrosion 


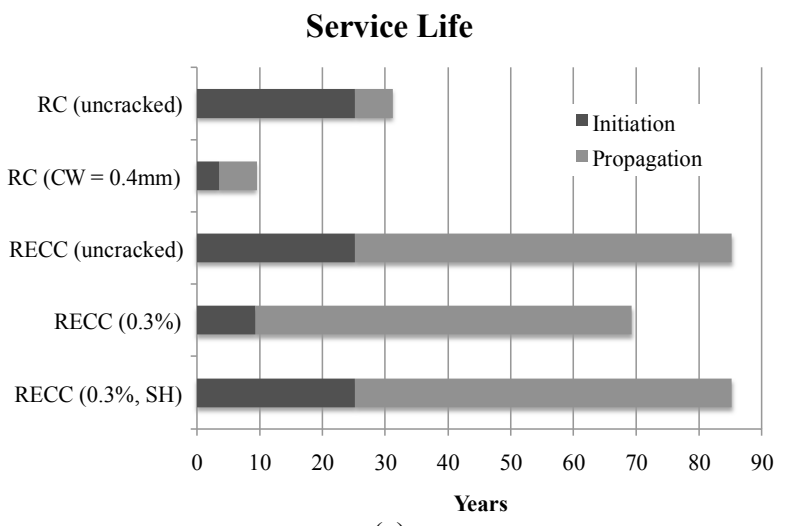

(a)

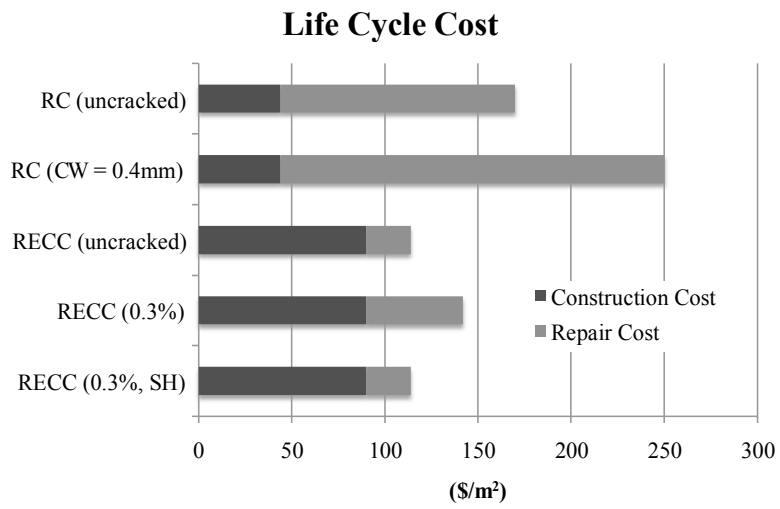

(b)

Fig. 6 Computed (a) service life and (b) life-cycle cost showing the effect of self-healing (Li et al, 2010). The "construction cost" here refers to mainly the initial material cost.

initiation $\mathrm{t}_{\mathrm{i}}$ for uncracked concrete from 8.9 years to 25.2 years. However, $\mathrm{t}_{\mathrm{i}}$ drops to 3.5 years when a crack of $400 \mu \mathrm{m}$ width is introduced. For the case of ECC, $t_{i}$ drops from 25.2 years to 9.3 years when a tensile strain of $0.3 \%$ is imposed but fully recovers to 25.2 years when self-healing takes place and completely seals the microcracks. The time to corrosion propagation of six years in normal concrete is extended to 60 years in ECC based on its strain-hardening ability. ECC is able to withstand the tension induced by steel rebar expansion as a result of corrosion, thus suppressing the spalling of the concrete cover (Lepech 2006). Through a combination of tensile ductility and self-healing ability, the total service life of the ECC bridge deck was shown to extend from less than ten years for the RC deck to over eighty years.

The calculated life-cycle costs (including materials cost and repair cost) for these cases are shown in Fig. 6b. Life-365 computes a life cycle cost of $\$ 170 / \mathrm{m}^{2}$ for $\mathrm{R} / \mathrm{C}$ when uncracked, but increases to $\$ 250 / \mathrm{m}^{2}$ when a crack of $400 \mu \mathrm{m}$ width is present. For the ECC bridge deck, the material cost assumed is higher $\left(\$ 90 / \mathrm{m}^{2}\right.$ compared with $\$ 44 / \mathrm{m}^{2}$ for normal concrete) reflecting the additional costs in ECC due to inclusion of fibers and other expensive ingredients. However, the repair cost for the ECC deck drops from $\$ 206 / \mathrm{m}^{2}$ for a concrete deck with $400 \mu \mathrm{m}$ cracks to only $\$ 52 / \mathrm{m}^{2}$ for a ECC deck with imposed $0.3 \%$ strain, and further down to $\$ 24 / \mathrm{m}^{2}$ when self-healing takes place. Thus, despite the higher initial material (construction) cost, the ECC bridge deck ends up with a lower life cycle cost of $\$ 114 / \mathrm{m}^{2}$, compared with $\$ 250 / \mathrm{m}^{2}$ for the RC deck with $0.4 \mathrm{~mm}$ crack width.

The above calculations highlight the economic value of self-healing for civil infrastructure. Self-healing translates into sealing of microcracks that reduces the effective diffusion coefficient for chloride ion transport, which in turn translates into longer corrosion initiation time and extended service life before repair is needed. Although the numerical values are based on a bridge deck in Michigan with focus on steel corrosion deterioration, the general model framework demonstrates the potentially significant economic impact of concrete with self-healing functionality.

The life cycle cost model described above is helpful in estimating service life extension and economic savings from reduced repair due to self-healing in concrete infrastructure. To assess the reduction of environmental and social impacts, a more comprehensive life-cycle assessment model is needed. The infrastructure sustainability framework (Fig. 7a) proposed by Keoleian et al. (2005) may be extended for this purpose. Input and output parameters are computed for each life cycle phase as shown in Fig. 7b. The self-healing functionality could be embedded into the module that quantifies life-cycle major and minor repair and maintenance events in the use phase, much as what has been described above for the life cycle cost model. In this way, the return loopings indicated by "Repair and maintenance" in Fig. 7b are reduced. This more comprehensive model will allow additional evaluation of sustainability metrics such as global warming potential, primary energy consumption, and motorist lost time associated with reconstruction events, in addition to agency costs. This research is being carried out at the University of Michigan.

\section{Robustness of self-healing}

To realize the goals of infrastructure sustainability highlighted above, it will be necessary to move beyond demonstrating the feasibility of self-healing in concrete. Self-healing will need to meet at least the following six criteria to assure robustness of this functionality and its intended purpose in real structures. Apart from quantifying self-healing robustness, these six criteria can also be used to evaluate the advantages and limitations of various approaches to achieving self-healing in concrete infrastructures.

\subsection{Six criteria for robustness}

Shelf life: Unlike many manufactured products, civil 


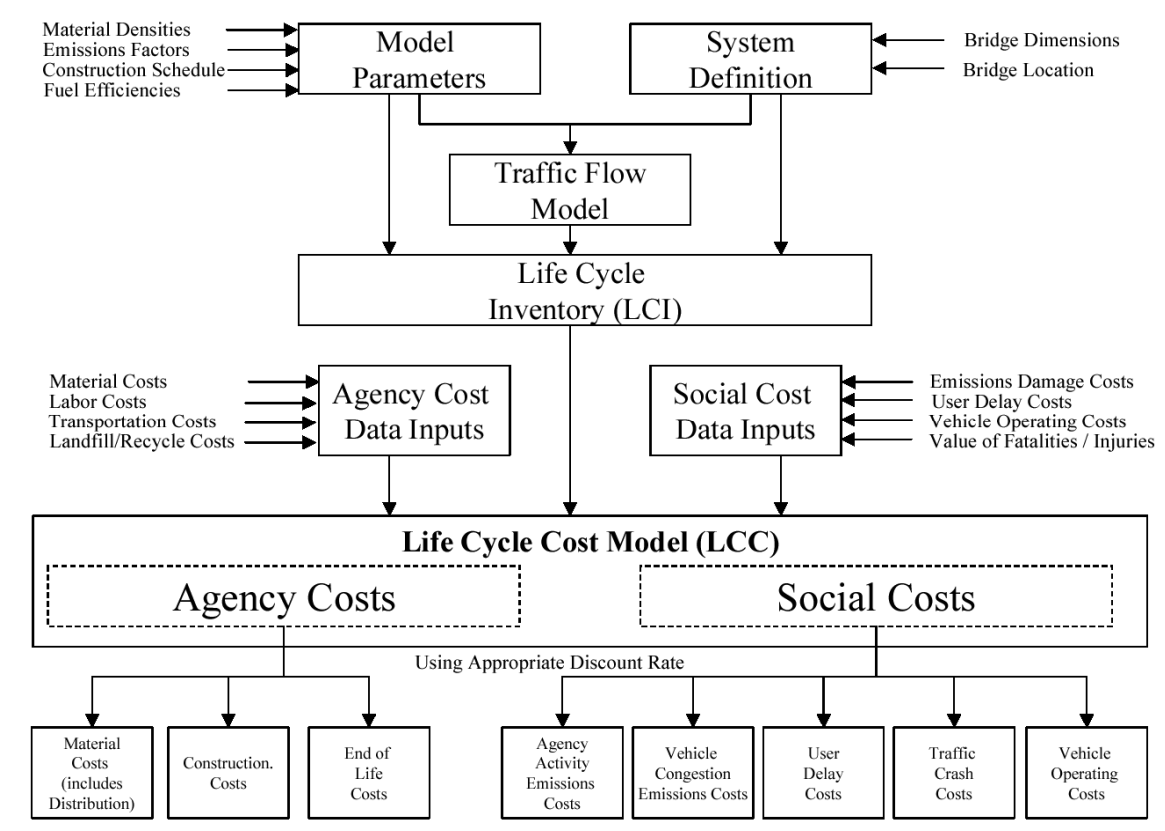

(a)

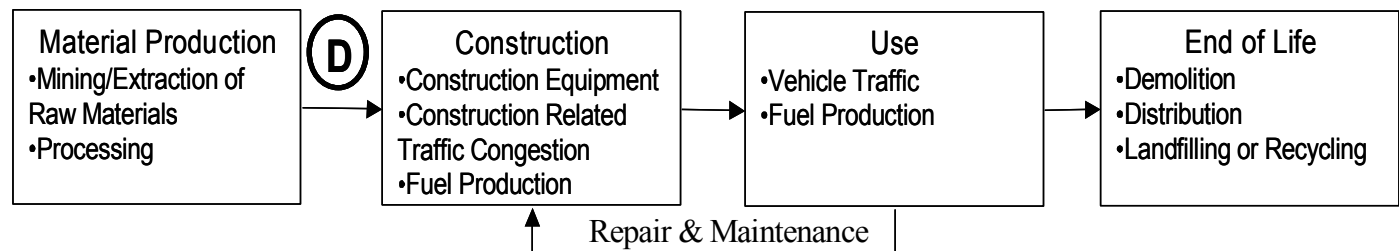

(b)

Fig. 7 (a) Integrated LCA-LCC Model (Keoleian et al 2005). Self-healing impacts on the infrastructure repair frequency and associated economic and environmental costs during the use phase of a life-cycle and reduces the loop back shown in (b).

infrastructure has a relatively long service life ranging from fifty to a hundred years. Since it is impossible to predict when damage of the concrete will occur over this period of time, it implies that any self-healing functionality must also possess a shelf life of fifty to a hundred years. This long shelf life requirement for infrastructure applications is possibly unique among engineered materials and products. This criterion indicates that the self-healing process, whether physical, biological, chemical or a combination of these, must possess a shelf-life that is as long as the design life of civil infrastructure.

Pervasiveness: Since most civil infrastructure experiences complex combined environmental and mechanical loading, it is prudent to assume that damage of the concrete may occur anywhere in the structure and that crack orientation may be difficult to predict accurately. This assumption implies that the self-healing functionality needs to be pervasive in the structure, rather than being available only in limited and discrete parts of a structural component. Cracks should be rehealed regardless of actual orientation.

Quality: Ideally, self-healing should lead to full re- covery of both transport and mechanical properties. Transport properties include permeability and diffusivity, while mechanical properties include stiffness, strengths and possibly ductility. If only transport properties are recovered, then the self-healing functionality is truly limited to self-sealing only. The percentage of recovery for a given level of damage also provides an indicator of the quality of healing. The quality criterion implies that the self-healing process must lead to products that not only fill the cracks, but also chemically bind the crack faces together.

Reliability: Self-healing reliability refers to the consistency of mechanical and/or transport property recovery. A lack of consistency from test to test (large coefficient of variation) would suggest the lack of reliability of the particular self-healing approach.

Versatility: Civil infrastructure is exposed to a variety of environments, some continuously dry, others continuously wet, and still others experience periods of dryness and wetness. They may also be exposed to a high chloride environment, such as bridge decks and roadways in northern climates where deicing salts are used, or in coastal regions where the infrastructure may 
come into contact with salt from seawater. Self-healing approaches that allow recovery of material properties in widely varying environments would be considered highly versatile.

Repeatability: Damage in a given civil infrastructure will likely take place repeatedly due to multiple overload events over the lifetime of the structure. Selfhealing approaches should therefore be able to function not just once, but multiple times over the design life of the structure. This criterion implies that the self-healing mechanism must remain operational when cracking occurs multiple times, even possibly in the same location of the structure.

The above six criteria - Shelf life, Pervasiveness, Quality, Reliability, Versatility, and Repeatability - may be used to evaluate the robustness of any given selfhealing approach. In most cases, current available experimental data are inadequate to conduct a quantitative evaluation. However, qualitative considerations of these six criteria with respect to current knowledge on the various self-healing approaches should still be useful to identify advantages and potential shortcomings that may require additional research efforts. This will be carried out in Section 3.3 after describing five generic approaches for self-healing.

\subsection{Self-healing approaches}

Self-healing approaches may be broadly grouped into five categories - chemical encapsulation, bacterial encapsulation, mineral admixtures, chemical in glass tubing, and intrinsic self-healing with self-controlled tight crack width. These approaches are schematically illustrated in Fig. 8. Related approaches that require human intervention, such as applying heat, are not considered here.

Chemical encapsulation (Fig. 8a) includes all approaches that utilize self-healing chemical agents contained in microcapsules that are dispersed uniformly in the concrete. While different chemicals and microcapsule materials have been investigated, the common
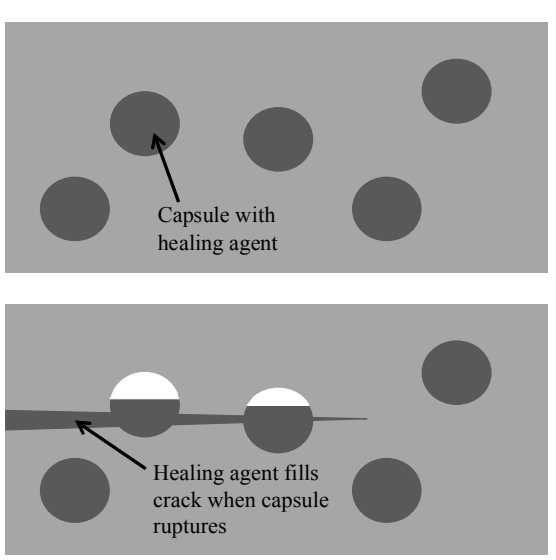

(a)
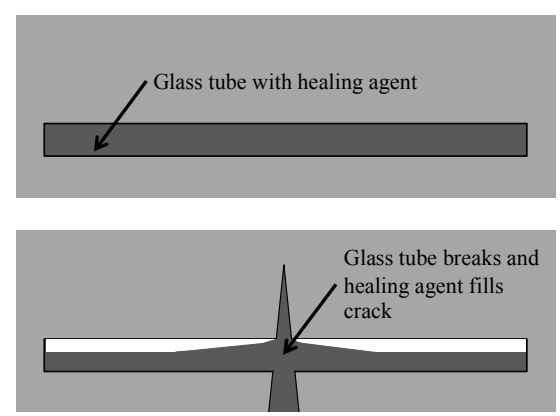

(d)

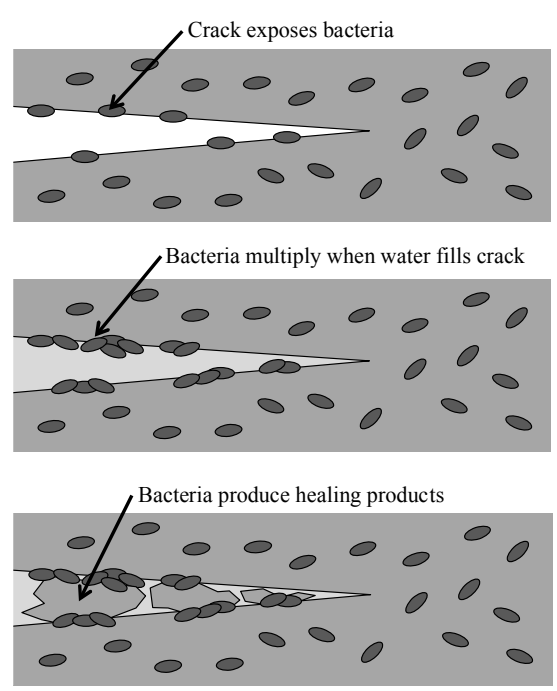

(b)
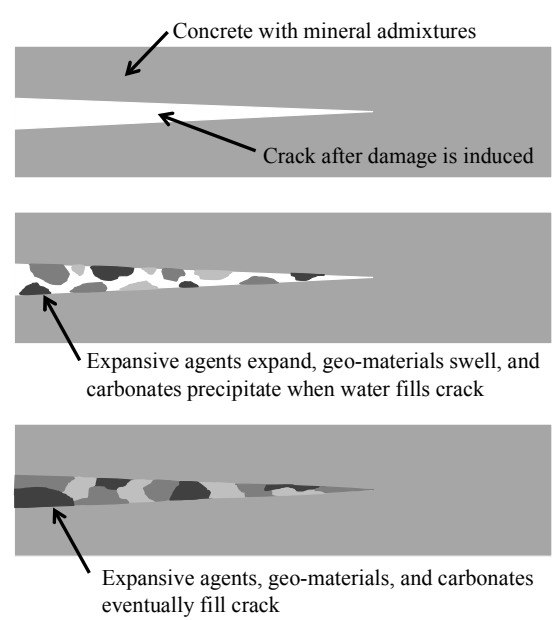

(c)

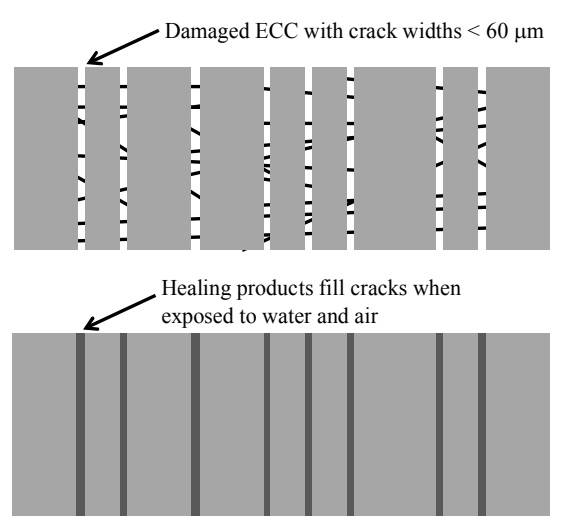

(e)

Fig. 8 Schematic illustration of (a) chemical encapsulation self-healing approach, (b) bacteria additive self-healing approach, (c) mineral admixtures self-healing approach, (d) glass tubing self-healing approach, (e) self-controlled tight crack width self-healing approach. 
theme is to isolate the healing chemical(s) from the concrete by the microcapsules until a concrete crack breaks them open. Leaking of the chemicals then either seals the crack and/or bonds the crack faces.

This approach is appealing due to the versatility of microencapsulation technology that can essentially encapsulate a variety of chemicals in any size down to submicron size. Huang and Ye (2011), for example, employed sodium silicate solution encapsulated in $5 \mathrm{~mm}$ wax capsules for self-healing in a cementitious composite which demonstrated recovery of mechanical properties including flexural stiffness and strength after damage induced by three-point bending tests.

The bacteria additive self-healing approach utilizes bacteria that induce precipitation of calcium carbonate as a result of carbonate generation by bacteria metabolism in a high calcium environment (Fig. 8b). The specific bacteria chosen must be able to withstand the high alkalinity of cement and the internal compressive pressure as microstructure continuously densifies with cement hydration. A nutrient must also be available to feed the bacteria. For example, Jonkers (2011) encapsulated spores of Bacillus pseudofirmus and Bacillus cohnii, calcium lactate and yeast extract in porous expanded clay particles up to $4 \mathrm{~mm}$ in size. Evidence of selfhealing was based on visual observation of calcium carbonate precipitates on the cracked surfaces after 100 days of immersion in water (Wiktor and Jonkers 2011). Wang et al. (2012) immobilized Bacillus sphaericus in silica gel and polyurethane inside $40 \mathrm{~mm}$ long glass tubes with $3 \mathrm{~mm}$ in diameter. Release of the bacteria upon glass tube fracturing caused by a mortar crack allows breaking down of urea into ammonium $\left(\mathrm{NH}_{4}{ }^{+}\right)$ and carbonate $\left(\mathrm{CO}_{3}{ }^{2-}\right)$. In the high calcium environment, calcium carbonate is precipitated on the bacterial cell wall and in the surrounding medium. Regaining of flexural strength and reduction of water permeability coefficient was reported as evidence of rehealing.

Mineral admixtures have been deployed as an approach for self-sealing of concrete cracks by reducing the water permeability after concrete damage (Fig. 8c). Kishi and co-workers (2007) demonstrated the use of a tailored mix of expansive agents $\left(\mathrm{C}_{4} \mathrm{~A}_{3} \overline{\mathrm{S}}, \mathrm{CaSO}_{4}\right.$, and $\mathrm{CaO}$ ), swelling geo-materials (mainly silicon dioxide and sodium aluminum silicate hydroxide, and montmorillonite clay) and various types of carbonates as partial $(10 \%$ by weight) cement replacement, and found that the sealing action can be effective for cracks up to $0.22 \mathrm{~mm}$ in water permeability tests in concrete with normal w/c ratio. Self-sealing phenomenon of cracks was confirmed by time-lapse optical microscopy, SEM and X-ray mapping on the rehealed crack zone, and by use of water permeability test under immersed condition (Ahn et al. 2010). The combined effects of geo-material swelling upon rehydration, expansive agent expansion and precipitation of carbonates in the crack were suggested as the mechanisms leading to effective (speedy formation and chemically stable healing products) heal- ing. Calcium sulfoaluminate (CSA) has also been utilized (Hosoda et al. 2007; Kishi et al. 2007; Sisomphon et al. 2011; Sisomphon and Copuroglu 2011) as an expansive agent for self-sealing.

The use of glass tubing for self-healing is based on the concept of self-sensing and actuation when a concrete crack is intercepted by the glass tubing which reacts by fracturing and releasing a repair chemical (Fig. 8d). (It should be noted, however, that this sensingactuation concept is in fact present implicitly or explicitly in all five self-healing approaches discussed here.) Indeed the glass tubing approach may be considered a variant of chemical encapsulation as an alternative form of healing agent delivery approach, with the advantage of potentially carrying a larger amount of healing agent compared with microcapsules. Various chemicals including methyl methacrylate (Dry and McMillan 1996), ethyl cyanoacrylate (Li et al. 1998; Joseph et al. 2010) and polyurethane combined with an accelerator (van Tittelboom et al. 2011) have demonstrated the ability of recovering concrete mechanical and transport properties. These chemicals are chosen to have low viscosity so that the healing agents can leak from the fractured glass tube into the concrete crack to perform the self-healing. As noted above, the healing agent is not limited to chemicals, but could be biological (Wang et al. 2012). Evidence of self-healing was based on mechanical reloading of beam elements that show a rebound of stiffness and/or strength (Li et al. 1998; Joseph et al. 2010; Van Tittelboom et al. 2011) and a reduction of permeability coefficient (Van Tittelboom et al. 2011).

One of the simplest approaches utilizes the intrinsic natural tendencies of continued hydration, pozzolanic, and carbonation processes when cracks in cementitious materials are exposed to water and carbon dioxide (in air), taking advantage of the existence of unhydrated cement grains and fly ash in a binder with low water/binder ratio. This approach can only work if the cracks are tight, $100 \mu \mathrm{m}$ or less. ter Heide and Schlangen (2007) demonstrated this approach by continued hydration of damaged concrete specimens placed under water and subjected to a compressive load that closes the cracks. $\mathrm{Li}$ and co-workers demonstrated the ability of self-healing in ECC that tensile strain-hardens with crack widths self-controlled to below $50 \mu \mathrm{m}$ (Fig. 8e). This tight crack width remains even when the material is loaded to several percent tensile strain. Evidence of self-sealing in ECC has been demonstrated by reduced permeability over time (Lepech and Li 2009). Evidence of self-healing in the mechanical sense has been demonstrated by recovery of stiffness in pre-damaged specimens (Sahmaran and Li 2008; Yang et al. 2009; Yamamoto et al. 2010; Li and $\mathrm{Li} 2011$ ) exposed to various environments.

\subsection{Self-healing robustness of various ap- proaches}

In this section, the five broad categories of self-healing 
approaches will be evaluated against the six criteria summarized in 3.1 above. Table 1 highlights the essence of the evaluation results. In performing this evaluation, it is realized that the current literature does not contain adequate data sets for proper assessment of reliability, as it has been typical that only a small number of specimens are used in all self-healing investigations so far.

As previously stated, chemical encapsulation is appealing due to its versatility. Not only can the capsules incorporate a variety of healing agents and be made in any size, but this approach could be used in a variety of infrastructures since the healing process is independent of the external environment. This approach also has the potential of a long shelf life, although this would depend on the chemical being encapsulated and how long it can remain active. Chemical encapsulation is also pervasive since the capsules could be mixed directly into concrete, provided they are strong enough to withstand the mixing process, thus making them uniformly dispersed in the matrix without being damaged. Huang and Ye (2011) demonstrated that this approach can be used to regain mechanical properties such as flexural stiffness and strength, but no work has been carried out to determine the recovery of transport properties. Also, although no studies have been conducted with more than one loading cycle, healing by chemical encapsulation is likely not repeatable since the capsules would be emptied during the first damage cycle unless repeated crack opening in the rehealed zone can be avoided.

Bacterial encapsulation has been used to completely regain transport properties in damaged concrete specimens (Jonkers 2011), but the regain in mechanical properties appears minimal (Wang et al. 2012). With this approach, the shelf life is determined by how long the bacterial spores are able to remain viable within the concrete matrix. Jonkers (2011) showed that encapsulated spores are viable for at least six months, and currently running viability tests will determine if there is a loss of viability over longer periods of time. This technique is also pervasive if the bacterial spores are incorporated into capsules that can be mixed directly into the concrete matrix. Currently it is unclear if the bacterial encapsulation self-healing approach possesses versatil-

Table 1 Robustness matrix of self-healing approaches.

\begin{tabular}{|c|c|c|c|c|c|}
\hline & $\begin{array}{c}\text { Chemical } \\
\text { Encapsulation }\end{array}$ & $\begin{array}{c}\text { Bacterial } \\
\text { Encapsulation }\end{array}$ & $\begin{array}{c}\text { Mineral } \\
\text { Admixtures }\end{array}$ & $\begin{array}{c}\text { Chemical in Glass } \\
\text { Tubing }\end{array}$ & $\begin{array}{c}\text { Intrinsic Healing } \\
\text { with } \\
\text { Self-Controlled } \\
\text { Tight Crack } \\
\text { Width } \\
\end{array}$ \\
\hline Shelf Life & $\begin{array}{l}\text { Potential of long } \\
\text { shelf life; would } \\
\text { depend on healing } \\
\text { agent in capsule }\end{array}$ & $\begin{array}{l}\text { Currently running } \\
\text { viability tests show } \\
\text { spores are viable for } \\
\text { at least } 6 \text { months }\end{array}$ & $\begin{array}{l}\text { Long shelf life as } \\
\text { long as additives } \\
\text { remain reactive and } \\
\text { unhydrated }\end{array}$ & $\begin{array}{c}\text { Potential of } \\
\text { long shelf life; } \\
\text { depends on } \\
\text { healing agent used }\end{array}$ & $\begin{array}{c}\text { Long shelf life } \\
\text { since no special } \\
\text { additives are incor- } \\
\text { porated into ECC } \\
\text { for } \\
\text { self-healing }\end{array}$ \\
\hline Pervasive & $\begin{array}{c}\text { Yes, capsules } \\
\text { could be } \\
\text { dispersed uniformly } \\
\text { in concrete }\end{array}$ & $\begin{array}{c}\text { Yes, capsules } \\
\text { containing spores } \\
\text { could be dispersed } \\
\text { uniformly in } \\
\text { concrete }\end{array}$ & $\begin{array}{l}\text { Yes, admixtures } \\
\text { are mixed in and } \\
\text { dispersed } \\
\text { uniformly in } \\
\text { concrete }\end{array}$ & $\begin{array}{l}\text { No, tubes have } \\
\text { to be placed in } \\
\text { discrete locations } \\
\text { where cracks and } \\
\text { crack orientation } \\
\text { are anticipated }\end{array}$ & $\begin{array}{c}\text { Yes, intrinsic } \\
\text { healing } \\
\text { mechanisms } \\
\text { available anywhere } \\
\text { in the structure for } \\
\text { any crack } \\
\text { orientation }\end{array}$ \\
\hline Quality & $\begin{array}{l}\text { Regains mechanical } \\
\text { properties to an } \\
\text { extent; self-sealing } \\
\text { has not been } \\
\text { investigated }\end{array}$ & $\begin{array}{l}\text { Complete self- } \\
\text { sealing } \\
\text { demonstrated; } \\
\text { regain of } \\
\text { mechanical } \\
\text { properties is } \\
\text { minimal }\end{array}$ & $\begin{array}{c}\text { Capable of } \\
\text { complete } \\
\text { self-sealing; } \\
\text { regain of } \\
\text { mechanical } \\
\text { properties has not } \\
\text { been investigated }\end{array}$ & $\begin{array}{l}\text { Self sealing and } \\
\text { regain of } \\
\text { mechanical } \\
\text { properties occur to } \\
\text { an extent, but not } \\
100 \% \text { efficient }\end{array}$ & $\begin{array}{l}\text { Self-sealing and } \\
\text { regain of } \\
\text { mechanical } \\
\text { properties can be } \\
100 \% \text { efficient }\end{array}$ \\
\hline Reliable & $\begin{array}{c}\text { Inadequate data } \\
\text { for proper } \\
\text { assessment }\end{array}$ & $\begin{array}{c}\text { Inadequate data } \\
\text { for proper } \\
\text { assessment }\end{array}$ & $\begin{array}{c}\text { Inadequate data } \\
\text { for proper } \\
\text { assessment }\end{array}$ & $\begin{array}{c}\text { Inadequate data } \\
\text { for proper } \\
\text { assessment }\end{array}$ & $\begin{array}{c}\text { Inadequate data } \\
\text { for proper } \\
\text { assessment }\end{array}$ \\
\hline Versatile & $\begin{array}{c}\text { Yes, healing } \\
\text { mechanism is inde- } \\
\text { pendent of external } \\
\text { environment }\end{array}$ & $\begin{array}{l}\text { Currently limited to } \\
\text { continuous water } \\
\text { exposure }\end{array}$ & $\begin{array}{c}\text { Currently } \\
\text { limited to } \\
\text { continuous water } \\
\text { exposure }\end{array}$ & $\begin{array}{c}\text { Yes, healing } \\
\text { mechanism is inde- } \\
\text { pendent of external } \\
\text { environment }\end{array}$ & $\begin{array}{c}\text { Yes, demonstrated } \\
\text { feasible in a variety } \\
\text { of exposure } \\
\text { environments }\end{array}$ \\
\hline Repeatable & $\begin{array}{l}\text { No studies have } \\
\text { been done with } \\
\text { more than one } \\
\text { loading cycle; likely } \\
\text { not repeatable }\end{array}$ & $\begin{array}{l}\text { No studies have } \\
\text { been done with } \\
\text { more than one load- } \\
\text { ing cycle }\end{array}$ & $\begin{array}{l}\text { No studies have } \\
\text { been done with } \\
\text { more than one } \\
\text { loading cycle }\end{array}$ & Not repeatable & $\begin{array}{l}\text { Yes, partial regain } \\
\text { in stiffness for sam- } \\
\text { ples exposed to } \\
\text { more than one } \\
\text { loading cycle }\end{array}$ \\
\hline
\end{tabular}


ity or repeatability characteristics since all studies to date are carried out under continuous water exposure, and no repeat loading has been applied. While experiments by Wiktor and Jonkers (2011) found that cracks up to 0.46 millimeters can be completely sealed with healing products, the versatility of this approach may be limited if the bacterial spores lose viability under drying conditions. Still, the bacterial encapsulation approach may be suitable for self-sealing of underground or water containing structures where continuous exposure of the concrete to water may be expected.

Self-healing using mineral admixtures is highly pervasive since the additives are used as partial cement replacements and are therefore dispersed uniformly throughout the concrete. Visual observation and permeability tests have shown that this approach allows for complete regain of transport properties (Kishi et al. 2007; Hosoda et al. 2007; Sisomphon et al. 2011; Sisomphon and Copuroglu 2011), but no research has been carried out to determine the feasibility of regain of mechanical properties. Currently it is unclear if this approach is versatile or repeatable; no investigation has been performed to determine the repeatability of this method and samples have only been allowed to heal in continuously wet environments. The mineral admixture self-healing approach appears to be intended for underground structures. The continuously wet environment for such structures would be inductive for this selfhealing technique. No specific work has been conducted to determine the shelf life, but there is potential for a long shelf life as long as sufficient mineral admixture particles remain unhydrated and reactive.

The main advantage of the glass tubing approach is that this technique can be quite versatile since the healing mechanism is independent of the external environment. Joseph and co-workers (2010) found that specimen age did not affect the healing response. Therefore, this approach could also provide a healing mechanism with a long shelf life, but this would depend on the healing agent used. The use of glass tubing is not pervasive since the tubes have to be placed in discrete locations where cracking is anticipated. In addition, the glass tubing must be oriented in a direction close to perpendicular to the anticipated crack path, so that the concrete crack would break the glass tube to activate the healing mechanism. Also, this technique is not repeatable since the glass tubes would most likely be emptied during the first loading cycle. It has been shown that self-healing using this approach leads to recovery of both transport and mechanical properties, however, it is not $100 \%$ efficient and the healing under repeated damage was minimal (Li et al. 1998; Joseph et al. 2010; Van Tittelboom et al. 2011).

Utilizing the intrinsic self-healing tendency of cementitious materials coupled with self-controlled tight crack width of ECC has proven to be a promising approach. Although no specific experiments have been conducted to determine the shelf life of this approach, it is likely that there would be a long shelf life since the intrinsic mechanisms of continuing hydration, pozzolanic reaction and carbonation in cementitious materials are known to be long lasting. This technique is highly pervasive since it utilizes unhydrated cement and fly ash, which are uniformly dispersed within the cementitious matrix. It has been found that the recovery of transport and mechanical properties can be $100 \%$ efficient depending on the preloading levels and exposure conditions (Lepech and Li 2009; Sahmaran and Li 2008; Yang et al. 2009; Yamamoto et al. 2010; Li and Li 2011). It has also been shown that this approach can be repeatable. Yamamoto et al (2010) found that there is a regain in stiffness for samples exposed to multiple damage and rehealing cycles. ECC self-healing has been demonstrated under continuous or intermittent water exposures, and at room and elevated temperatures (Yang et al. 2009) and at young and mature ages (Yang et al. 2009; Yang et al. 2011). In addition, ECC has been shown to self-heal under various adverse conditions, such as highly alkaline and chloride environments (Sahmaran and Li 2008; Li and Li 2011).

\section{Field studies on self-healing ECC}

Limited research has been conducted to determine the level of self-healing under field conditions. Although self-healing in the lab may look promising, these results have limited value unless the self-healing techniques discussed above can be applied to structures in the field.

Kishi et al (2011) conducted field tests to determine the recovery of water tightness due to self-healing in water-retaining containers. The concrete, which contained mineral admixtures for self-healing, was prepared in a ready-mix concrete plant and delivered to the casting site in an agitator truck. The concrete was then cast into box shaped water-retaining containers and, after several months of curing, cracks of 0.2 millimeters were induced and the boxes were filled with water. The amount of water leakage over time was measured to determine the extent of self-healing. It was found that the fresh properties of the self-healing concrete produced at the ready-mix plant satisfied construction field requirements and the mineral admixtures within the concrete helped seal the cracks and reduce water leakage from the containers.

Herbert and Li (2011) performed a three-month study on the self-healing of ECC in the natural environment, where samples were exposed to random and sometimes extreme environmental conditions. Through visual observation, it was found that the majority of cracks less than 20 micrometers in width underwent self-healing. In contrast, cracks up to 150 micrometers have been able to heal under controlled laboratory conditions (Yang et al. 2009). It was also found that ECC samples in the natural environment were able to recover a significant portion of their initial stiffness after three months of natural environment exposure, but this recovery was 
less than that for samples healed in the laboratory. Therefore, although self-healing of ECC in the natural environment is promising, it is not as robust as the selfhealing seen under controlled laboratory conditions.

\section{Further discussions and conclusions}

From a holistic viewpoint, the recent technological developments in self-healing concrete and the serious deterioration of civil infrastructure on a broad scale produce a convergence of technology push and pull that should result in future smart infrastructure with the intrinsic ability to self-heal when damaged. The success of this new technology will transform the current civil infrastructure practice of deterioration-repair cycles to one of health self-maintenance without external intervention such as inspection and repair. The implication of selfhealing infrastructure on economic impact, environmental sustainability, and quality of life is expected to be significant.

All five self-healing approaches - chemical encapsulation, bacterial encapsulation, mineral admixtures, chemical in glass tubing, and intrinsic healing with selfcontrolled tight crack width, have been demonstrated to be effective to some extent under certain laboratory conditions. However, there are limitations in almost all approaches that will require additional investigation before the vision of truly self-repairing civil infrastructure can be fully realized. In some cases, the limitations are intrinsic to the fundamental nature of the selfhealing approach so as to make it impossible to attain robustness.

For the chemical encapsulation approach, almost all the robustness criteria could be met in principle, although substantial additional research will be needed to verify this expectation quantitatively. A significant advantage of this approach is the continuing maturation of technology for micro-encapsulation of a wide range of chemicals for self-healing (see, e.g. Yang et al. 2008). Perhaps the most severe limitation is the difficulty in meeting the repeatability criterion since once emptied, the capsules are not likely to be usable for repair during the next cycle of damage. This may imply that selfhealing can take place just once for damage in the same location of a structure. On the other hand, if the microcapsules are truly on the size-scale comparable to cement grains, then there may be enough of them to persist in self-healing functionality even under multiple damage events. The challenge would be to assure filling the crack volume given the limited amount of healing chemicals that could be encapsulated in such microcapsules especially in light of mm-size cracks commonly observed in concrete infrastructure.

For the bacterial encapsulation approach, the adoption of biological processes for concrete self-healing could be highly efficient and attractive. However, it is not known if the bacterial spores are able to remain viable during the entire service life of civil infrastructure, especially if the structure is not under a constantly wet environment. These considerations of shelf life and versatility may limit the applicability of this approach to certain types of structures such as underground or water retaining infrastructure.

For the mineral admixtures approach, demonstration of the effectiveness of sealing cracks and reducing water leakage has been highly convincing. An outstanding feature of this approach is the ability to self-heal cracks of relatively large width, up to $0.22 \mathrm{~mm}$. This is one of the few approaches that have undergone limited fieldtesting (Kishi et al. 2011). The most urgent research needs here include the verification of true self-healing in the sense of recovery of mechanical properties to levels seen before concrete damage, and verifying that rehealing under repeated damage is feasible.

For the chemical in glass tubing approach, the demonstration of mechanical property recovery and the flexibility of adopting different types of self-healing chemicals in the glass tube make it promising. The strongest limitations, however, are the nonpervasiveness of this approach unless a network of such tubes is used throughout the structure. The fracturing of the glass tube to activate the self-healing mechanism also limits the repeatability of this approach. Glass tubes and capsules are simply different geometric forms of vessels containing the repair agent. As microencapsulation technology continues to mature, it is likely that the limitations of the glass tubing approach would yield to the advantages of microencapsulation.

The intrinsic healing with self-controlled tight crack width approach (based on ECC) shows promise of meeting all six robustness criteria. However, the economic cost of ECC is at present substantially higher (two to three times) than that of normal concrete. As shown in Section 2, the higher initial cost of ECC could be offset by the lower life-cycle maintenance cost, so that this self-healing approach could be justified on an economic life cycle cost basis. In addition, the tight crack width and tensile ductility of ECC serves other purposes of infrastructure durability and resiliency enhancements.

It may be of interest to investigate combinations of the five different approaches to lead to further enhancements in self-healing. Already, encapsulation methods have been used for both chemicals and bacteria. In future, it is conceivable that highly robust selfhealing could be attained by combining bacteria and/or expansive additives in ECC with tight crack width control.

The lack of data to properly evaluate the reliability of the various self-healing approaches points to the need for further research with a larger number of specimens.

Perhaps the biggest adversary of self-healing is large crack width. As a tension-softening material, normal concrete is difficult to reheal since the crack width can extend indefinitely. Even with steel reinforcement, it is generally agreed that crack width in concrete structures is difficult to control. This point is driven home by the 
change in code language between the 1995 edition (ACI 318R-95) and the 2002 edition (ACI 318R-02) of the ACI Building Code. The specification (in the 1995 edition) of the Gergely-Lutz " $z$ " factor in steel reinforcement for limiting the crack width to a recommended value of $400 \mu \mathrm{m}$ for exterior exposure conditions was removed. In its place was the specification in the 2002 edition of maximum reinforcement spacing "intended to limit surface cracks to a width that is generally acceptable in practice but may vary widely in a given structure". As crack width increases, the efficiency of selfhealing drops rapidly. ECC has definite advantage in attaining higher self-healing efficiency by virtue of intrinsic tight crack width control in the material, without dependence on steel reinforcement.

As pointed out in Section 4, very little work has been conducted in self-healing under a natural environment. Conditions in the field such as highly variable temperature and precipitation may render self-healing that looks promising under highly controlled conditions in the laboratory meaningless. This is an area of research that should receive much more attention in the future, if the objective is to realize extended service life and to reduce economic, social and environmental life-cycle costs for civil infrastructure. Given the trajectory of research and progress made over the last decade, selfhealing sustainable concrete infrastructure appears to be a realistic expectation in the recent future provided that the six self-healing robustness criteria are met with additional research and validated under field conditions.

\section{References}

AASHTO, (2004). "LRFD Bridge Design Specifications." 3rd Ed., AASHTO, Washington, D.C, Ahn, T. H. and Kishi, T., (2010). "Crack self-healing behavior of cementitious composites incorporating various mineral admixtures." Journal of Advanced Concrete Technology, 8(2), 171-186.

American Concrete Institute, (1995). "Building code requirements for structural concrete (ACI 318-95) and commentary (ACI 318R-95)." ACI Committee 318, Detroit, Michigan,

American Concrete Institute, (2002). "Building code requirements for structural concrete (ACI 318-02) and commentary (ACI 318R-02)." ACI Committee 318, Detroit, Michigan.

Dry, C, and McMillan, W., (1996). "Three-part methylmethacrylate adhesive system as an internal delivery system for smart responsive concrete." Smart Mater Struct, 5(3), 297-300.

Herbert, E. N. and Li, V. C., (2001). "Self-healing of engineered cementitious composites in the natural environment." In: High Performance Fiber Reinforced Cement Composites 6, Ann Arbor, MI, USA, June 20-22.

Hosoda, A., Kishi, T., Arita, H. and Takakuwa, Y., (2007). "Self healing of crack and water permeability of expansive concrete." In: 1st International
Conference on Self-Healing Materials, Noordwijk aan Zee, The Netherlands, April 18-20.

Huang, H., and Ye, G., (2011). "Application of sodium silicate solution as self-healing agent in cementitious materials." International Conference on Advances in Construction Materials through Science and Engineering, Hong Kong, China, September 5-7.

Jonkers, H. M., Thijssen, A., Muyzer, G., Copuroglu, O. and Schlangen, E., (2010). "Application of bacteria as self-healing agent for the development of sustainable concrete." Ecological Engineering 36, 230-235.

Jonkers, H. M., (2011). "Bacteria-based self-healing concrete." Heron, 56(1/2), 1-12.

Joseph, C., Jefferson, A. D., Isaacs, B., Lark, R. and Gardner, D., (2010). "Experimental investigation of adhesive-based self-healing of cementitious materials." Magazine of Concrete Research, 62(11), 831-843.

Keoleian, G. A., Kendall, A., Chandler, R., Helfand, G. E., Lepech, M. and Li., V. C., (2005). "Life cycle cost model for evaluating the sustainability of bridge decks." In: Andrzej S. Nowak \& Dan M. Frangopol, Eds., Proc. Advances in Life-Cycle Analysis \& Design of Civil Infrastructure Systems, 143-150.

Kishi, T., Ahn, T., Hosoda, A., Suzuki, S. and Takaoka, H., (2007). "Self-healing behaviour by cementitious recrystallization of cracked concrete incorporating expansive agent." In: $1^{\text {st }}$ International Conference on Self-Healing Materials, Noordwijk aan Zee, The Netherlands, April 18-20.

Kishi, T., Ahn, T. H., Morita, M. and Koide, T., (2011). "Field test of self-healing concrete on the recovery of water tightness to leakage through cracks." In: $3 r d$ Int'l Conf. on Self-Healing Materials, Bath, UK 2011.

Lepech, M. D., (2006). "A paradigm for integrated structures and materials design for sustainable transportation infrastructure." $\mathrm{PhD}$ Thesis, University of Michigan.

Lepech, M. D. and Li, V. C., (2009). "Water permeability in engineered cementitious composites." Cement and Concrete Composites, 31(10), 744-753.

Li, M., and Li, V. C., (2011). "Cracking and healing of engineered cementitious composites under chloride environment.” ACI Materials J., 108(3), 333-340.

Li, M., Ranade, R. Kan, L. and Li, V. C., (2010). "On improving the infrastructure service life using ECC to mitigate rebar corrosion." In: van Breugel, K. Ye, G. and Yuan, Y. eds. Proc.,2nd Int'l Symp. on Service Life Design for Infrastructure, Delft, The Netherlands, RILEM PRO 70, 773-782.

Li, V. C., (2003). "On engineered cementitious composites (ECC) - A review of the material and its applications.” J. Advanced Concrete Technology, 1(3) 215-230.

Li, V. C., Lim, Y. M. and Chan, Y., (1998). "Feasibility study of a passive smart self-healing cementitious composite." Composites Part B: Engineering, 29B, 819-827. 
Li, V. C., Wu, C., Wang, S., Ogawa, A. and Saito, T., (2002). "Interface tailoring for strain-hardening PVAECC.” ACI Materials J., 99(5) 463-472.

Life-365, 2012. http://www.life-365.org/installation.html, Assessed March $2^{\text {nd }}, 2012$.

Mather, B. and Warner, J., (2003). "Why do concrete repairs fail." Interview held at Univ. of Wisconsin, Dept. of Eng. Professional Development, MD, WI, $<$ http://aec.engr.wisc.edu/resources/rsrc07.html $>$. Accessed, Nov., 2003.

RILEM TC-221-SHC, (2012). "State-of-the-art report Self-healing phenomena in cement-based materials, Ed. by de Rooij et al.

Sahmaran, M., Li, M. and Li, V. C., (2007). "Transport properties of engineered cementitious composites under chloride exposure." ACI Materials J., 104(6) 604-611.

Sahmaran, M. and Li, V. C., (2008). "Durability of mechanically loaded engineered cementitious composites under highly alkaline environments." Cement and Concrete Composites, 30, 72-81.

Sisomphon, K., Copuroglu, O. and Koenders, E. A. B., (2011). "Surface crack self healing behaviour of mortars with expansive additives." In: $3^{\text {rd }}$ International Conference on Self-Healing Materials, Bath, UK, June 27-29.

Sisomphon, K. and Copuroglu, O., (2011). "Self healing mortars by using different cementitious materials." In: International Conference on Advances in Construction Materials through Science and Engineering, Hong Kong, China, September 5-7.

ter Heide, N. and Schlangen, E., (2007). "Self-healing of early age cracks in concrete." In: Proceedings of the First International Conference on Self Healing Materials, 18-20 April 2007, Noordwijk aan Zee, The Netherlands.

Wang, J., van Tittelboom, K. De Belie, N. and Verstraete, W., (2012). "Use of silica gel or polyurethane immobilized bacteria for self-healing concrete." Construction and Building Materials, 26, 532-540.
Wiktor, V. and Jonkers, H. M., (2011). "Quantification of crack-healing in novel bacteria-based self-healing concrete." Cement and Concrete Composites, 33, 763-770.

Wu, M., Johannesson, B. and Geiker, M., (2012). “A review: Self-healing in cementitious materials and engineered cementitious composite as a self-healing material." Construction and Building Materials, 28, 571-583.

van Breugel, K., (2007). "Is there a market for selfhealing cement-based materials." In: Proceedings of the first international conference on self-healing materials, Noordwijk aan zee, the Netherlands; April 2007.

van Tittelboom, K., De Belie, N., Van Loo, D. and Jacobs, P., (2011). "Self-healing efficiency of cementitious materials containing tubular capsules filled with healing agent." Cement and Concrete Composites, 33, 497-505.

Vision 2020, (2006). " $A$ vision for the concrete repair protection and strengthening industry." The Strategic Development Council (SDC. http://www.concretesdc. org/_pdfs/Vision2020-Version1.0_\%20May2006.pdf

Yang, J., Keller, M. W., Moore, J. S. White, S. R. and Sottos, N. R., (2008). "Microencapsulation of isocyanates for self-healing polymers." Macromolecules, 41, 9650-9655.

Yang, Y., Lepech, M. D., Yang, E. and Li, V. C., (2009). "Autogenous healing of engineered cementitious composites under wet-dry cycles." Cement and Concrete Research, 39, 382-390.

Yang, Y., Yang, E. H. and Li, V. C., (2011). "Autogenous healing of engineering cementitious composites at early age." Cement and Concrete Research, 41(2), 176-183.

Yamamoto, A., Watanabe, K., Li, V. C. and Niwa, J., (2010). "Effect of wet-dry condition on self-healing property of early-age ECC." Proc. Japan Concrete Institute, 32(1) 251-256. 


\title{
Development of High-Durability Concrete with a Smart Artificial Lightweight Aggregate
}

\author{
Jinhwan Jeon $^{1}$, Tetsushi Kanda ${ }^{2}$, Haruki Momose ${ }^{2}$ and Hirozo Mihashi ${ }^{3}$
}

\begin{abstract}
Micro-crack development associated with the aging of concrete structure may be concerned in terms of decrease in durability. This study developed a smart artificial lightweight aggregate (ALA) capable of preventing micro-cracks by the internal curing with a reactive solution housed in its pore spaces. Water releasing capability of the smart ALA was first examined through laboratory tests that confirmed the improvement of water retaining capability within concrete. It was shown that water diffusion in concrete with the smart ALA was largely delayed leading to a decrease in micro-crack development and increase in compressive strength and improvement of durability indexes such as carbonation depth and air permeability.
\end{abstract}

\section{Introduction}

Concrete structures are subjected to drying and aging resulting in cracking and a subsequent decrease in its durability. Environmental conditions in cover concrete drive water transport and the resulting drying causes the visible and micro-cracks. One appropriate choice for the durable structures may be to achieve cover concrete with mitigating those cracks. The cracks need to be controlled by means of sufficient curing after placing but long term curing is very difficult in practice. To compensate the effects of long term curing, concrete-internal curing with water entraining media involved in concrete may mitigate these cracks and leads to achieving durable concrete structures.

Internal curing has been recently investigated to reduce autogenous shrinkage in high performance concrete (HPC, hereafter). HPC often suffers from early age cracking due to autogenous shrinkage, and this early-age cracking phenomena have been extensively investigated for years (e.g., Jensen and Hansen 2001). It has been proposed that HPCs' autogenous shrinkage and early age cracking can be reduced by introducing concrete-internal curing by involving water entraining media as one of significant countermeasures for the phenomena (e.g., RILEM 2007, Jensen and Hansen 2001, Jensen and Lura 2005). The most typical water absorbed medias is artificial lightweight aggregate (ALA). Henkensiefken et al. (2009) demonstrated that saturated ALA could nicely

\footnotetext{
${ }^{1}$ Research engineer, Building Construction and Materials Group, Kajima Technical Research Institute, Tokyo, Japan.

E-mail:jeon@kajima.com

${ }^{2}$ Research engineer, Building Construction and Materials Group, Kajima Technical Research Institute, Tokyo, Japan.

${ }^{3}$ Professor emeritus, Tohoku University, Sendai, Japan.
}

mitigate autogenous shrinkage.

Technology for durable and crack-mitigating concrete cover should be based on normal strength concrete rather than HPCs to be widely accepted in construction industry. This is because cost of HPCs reaches 2 to 3 times higher than normal concrete typically in Japan, and durable cover concrete with normal strength may be at most 2 times higher in cost even if some supplemental constituent materials are added. Very few studies exist utilizing internal curing in normal strength concrete to achieve durable cover concrete. Crack mitigating effects of ALA's concrete-internal curing on normal concrete have not been clarified. Furthermore, an expecting obstacle to adopt this approach is that saturated ALA rapidly loses absorbed water as ambient $\mathrm{RH}$ decreasing (Tsuchiya et al. 2010). Typical concrete cover with normal lightweight concrete involving ALA cannot take advantage of internal curing effects. This is because internal water content in the lightweight concrete cover rapidly decreases with time and therefore internal curing effects may diminish. This implies that ALA's water retaining characteristics should be modified to achieve durable concrete cover with normal concrete.

A goal in this study is to achieve durable concrete by developing a smart ALA, which is capable of preventing micro-cracks and densifies concrete microstructure by internal curing with a reactive solution housed in its pore spaces. The smart ALA is expected to bust the aforementioned obstacles. To reach this goal, two types of smart ALAs are produced, whose water desorption characteristics, water diffusion characteristics are experimentally investigated in comparison with distilled water saturated ALA. Then the effects of the smart ALA's internal curing are demonstrated in terms of increasing compressive strength, slowing carbonation progress, and decreasing micro-cracking amount in concrete. 


\section{ALA as a smart material}

\subsection{Basic concept}

Basic concept of smart ALA is demonstrated in Fig. 1. Smart ALA is aimed at developing highly durable concrete cover capable of preventing micro-cracks and densifies concrete microstructure by internal curing with a reactive solution housed in its pore spaces. We adopt four steps to reach this goal to take advantage of more effective internal curing using smart ALAs. In the first step, ALA type suitable for effective internal curing is selected in this study. Adopted ALA can absorb less water in desorption process than normal ALAs but delay rapid retaining water releasing in drying condition in concrete. This delay is expected to contribute to ease the aforementioned obstacle to use internal curing concept to normal strength concrete. For high strength concrete, the adopted ALA has been successfully used to mitigate autogenous shrinkage in practice (Hishiki et al. 2005).

In the second step, reactive chemical solution replaces water housed in ALAs for efficient internal curing and densifying concrete microstructure. This chemical solution selected in this study is colloidal silica, which is alternatively called as ultra-fine silica powder solution or nano- $\mathrm{SiO}_{2}$. Colloidal silica solution has 20 times higher viscosity than water and hence is going to strengthen function of smart ALAs, where enhances smart ALAs' retaining water content and delaying retaining water release in desorption isotherm. Furthermore, colloidal silica released from the smart ALAs reacts with calcium hydroxide (pozzolanic reaction) and densifies concrete

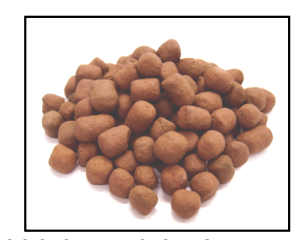

Artificial Light-weight Aggregate(ALA)

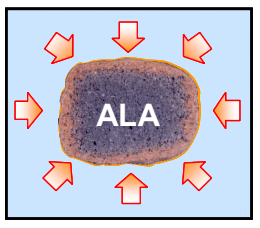

Impregnation of Chemical Solution

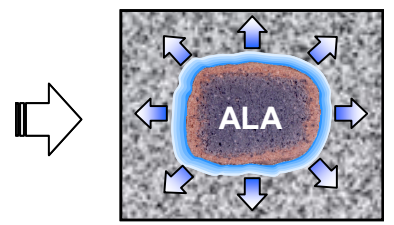

Diffusion of Chemical Solution to the Cement Matrix
Fig. 1 Schematic concept of smartize artificial light-weight aggregate. microstructure. This reaction has been reported to improve concrete's microstructure and permeability (e.g., Ji 2005), and may fill micro-crack formed in concrete due to drying. The colloidal silica may even heal nucleated micro-cracks due to the pozzolanic reaction, which is similar to self-healing function in other studies (e.g., Ahn and Kishi 2010). In the third step, viscosity controlled colloidal silica is used, which is modified by adding sodium bicarbonate solution. This addition leads to forming silica gel in ALAs' pores and is expected to further increase retaining water content and delaying retaining water release. The final step is to mix fly ash in concrete in addition to the smart ALAs to strengthen microstructure densification.

\subsection{Experimental plan}

Testing items and types of impregnated solutions for smart ALAs are shown in Table 1. Major components of adopted ALA with an absolute-dry density of $1.53 \mathrm{~g} / \mathrm{cm}^{3}$ (maximum water content is 18.0 mass\%) are coal ash and fine shale powder (Kasai et al. 2008). Properties of the adopted ALA are presented compared with a Japanese typical regular ALA product in Table 1. The adopted ALA has half of pore volume, 2/3 of water absorption rate, 2.5 times of crushing strength compared with of the regular ALA in this table. The adopted ALA has dense shell and rather porous inside in section. A scanning electron micrograph of the ALA cross section is shown in Fig. 2, where denser shell part is demonstrated than inside. Smaller pore volume in the adopted ALA has been presented than the regular ALA in Fig. 3. This figure shows the former has much less pore volume ranging from 0.1 to $1 \mu \mathrm{m}$ than the latter. This pore structure is expected to contribute to better internal curing effects for normal strength concrete.

Three types of impregnated solutions were used as shown in Table 2: distilled water, colloidal silica solution $\mathrm{A}$ and a viscosity-controlled colloidal silica solution B,

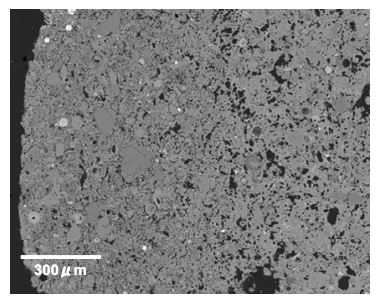

Surface

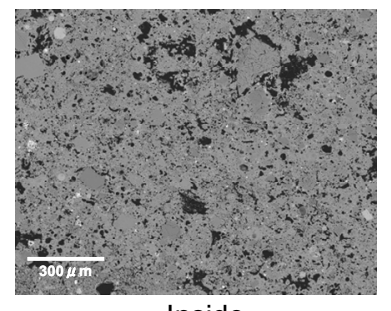

Inside
Fig. 2 SEM BSE image of the artificial light-weight aggregate cross section.

Table 1 Property of adopted ALA.

\begin{tabular}{|c|c|c|c|}
\hline Property & Adopted ALA & Regular ALA & Testing \\
\hline Density in oven-dry condition $\left(\mathrm{g} / \mathrm{cm}^{3}\right)$ & 1.53 & 1.29 & JIS A 1135 \\
\hline Water absorption rate (weight $\%$ ) & 18.0 & 26.0 & JIS A 1135 \\
\hline Crashing Strength (N) & 1510 & 576 & JIS Z 8841 \\
\hline Total pore Volume $(\mathrm{ml} / \mathrm{g})$ & 0.162 & 0.391 & - \\
\hline
\end{tabular}


Table 2 Test items and types of impregnated solutions.

\begin{tabular}{|c|c|c|}
\hline & $\begin{array}{c}\text { Type of impregnated } \\
\text { solutions }\end{array}$ & Test Items \\
\hline DW & Distilled water & \multirow{3}{*}{$\begin{array}{l}\text { Rate of impregnation } \\
\text { Isothermal diffusivity }\end{array}$} \\
\hline Sol.A & Colloidal silica & \\
\hline Sol.B & Controlled colloidal silica & \\
\hline
\end{tabular}

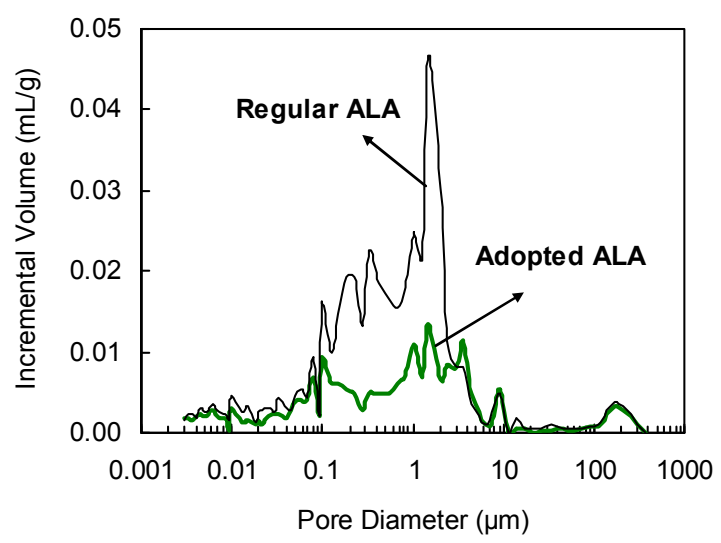

Fig. 3 Pore size distribution of adopted ALA compared with regular ALA.

which are corresponding to the first to third steps explained in a previous section. Solution A is a colloidal silica solution product in a market, whose average particle diameter is 10 to $20 \mathrm{~nm}$, solid content is $30 \%, \mathrm{pH}$ is 10 , viscosity is $15 \mathrm{cps}$ at $25^{\circ} \mathrm{C}$, specific gravity is 1.21 at $25^{\circ} \mathrm{C}$. Solution B is produced from solution A by adding $0.2 \%$ sodium bicarbonate solution. This addition leads to gel formation in ALA, which appears to be caused by colloidal silica's condensation polymerization. We confirmed this gel formation phenomenon in preliminary test but have not yet clarified detailed chemical process.

Impregnated solution content was measured to clarify the degree of impregnation and water transport properties, while isothermal drying test was performed to check the transport of solution under various relative humidities. Absolute-dry ALA was introduced to a vacuum desiccator in contact with three solutions mentioned above until a constant mass was confirmed. The impregnated ALA was wiped, weighed and the difference between the absolute-dry mass was divided with the absolute-dry mass as a mass-based impregnated solution content.

The isothermal desorption test was performed with a flow-division humidity generator. Impregnated ALAs were introduced to the specimen chamber, where they were dried under a controlled air flow of 10 liters per minute (variation of $\pm 1 \%$ ) with a specified relative humidity at a temperature of $20^{\circ} \mathrm{C}$, and changes in mass were continuously recorded. Although this method mainly evaluates water transport in gas phase and is different from that in concrete, it is still valid in comparative evaluation of the water transport of smart ALAs in concrete.

\subsection{Impregnated solution content and water diffusivity}

Because the maximum impregnated solution content was nearly 18 mass percent regardless of the solution type, the vacuum saturation method was found to be useful enough to attain the solution content as high as that of distilled water. The masses of the specimens with an impregnated solution showed gradual decrease with time. Specimens with solution A and B showed slower desorption than that with distilled water. The equilibrium impregnated solution content and diffusivity of solutions were simultaneously determined by curve-fitting the desorption data with Eq. 1 (Tada and Watanabe 2005). The equilibrium impregnated solution content corresponds to the equilibrium moisture content when the adsorbate is water.

$$
m(t)=a[1-b \exp (-c t)]
$$

where $\mathrm{m}(t)$ is the total amount of mass entering or leaving the specimen $(\mathrm{kg})$ at a time $t(\mathrm{~s}), a(\mathrm{~kg})$ is the equilibrium impregnated solution content after the infinite laps of time, $b$ is a coefficient regarding to the shape of specimen (sphere in this case) and $c(1 / \mathrm{s})$ is a coefficient including the solution diffusion coefficient $D_{\theta}\left(\mathrm{m}^{2} / \mathrm{s}\right)$ and is defined as $\mathrm{c}=D_{\theta} \pi^{2} / A^{2}$, where $A$ is the radius of the specimen in millimeter. Because $a$ represents the estimated equilibrium impregnated solution content. $\theta_{\mathrm{e}}$ $\left(\mathrm{kg} / \mathrm{m}^{3}\right)$ during desorption at a relative humidity, the residual amount of impregnated solution in the specimen $\theta_{\mathrm{r}}\left(\mathrm{kg} / \mathrm{m}^{3}\right)$ can be given by the following equation.

$$
\theta_{r}=\theta_{\max }-\theta_{e}
$$

where. $\theta_{\max }\left(\mathrm{kg} / \mathrm{m}^{3}\right)$ is the maximum solution content obtained with the vacuum saturation method.

The relationship for the equilibrium impregnated solution content as a function of relative humidity is shown in Fig. 4. This figure shows overall trend in which the equilibrium impregnated water or solution contents decreased with a decrease in relative humidity, but two remarkable findings. First, the effects of ALA types are

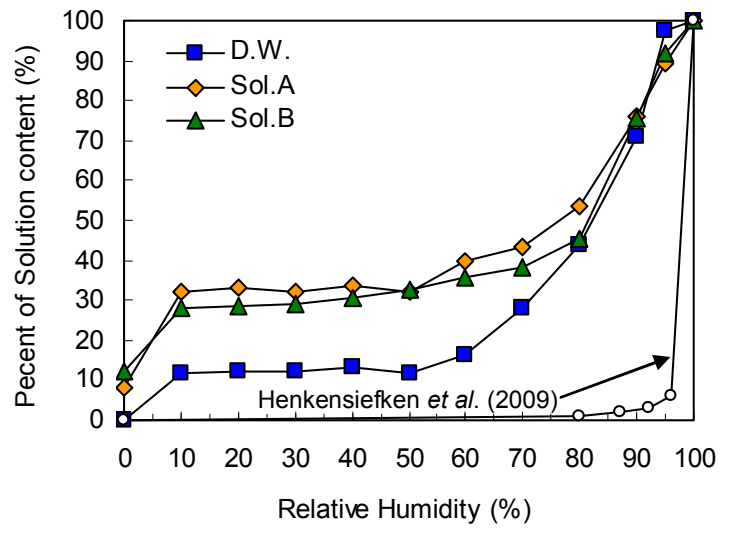

Fig. 4 Relationship between solution content and relative humidity on diffusion. 
significant. The equilibrium impregnated distilled water content in the adopted ALA is dramatically increased in any relative humidity revel compared with reference ALA referred from Henkensiefken et al. 2009. However, it should be noted that the reference ALA's properties are not clear and their effects on this water retaining capability is not clarified. When it is hypothesized that the regular ALA in Table 1 is similar to the reference ALA in Fig. 4, pore volume in the size from 0.1 to $1 \mu \mathrm{m}$ may dominate this water retaining capability. Confirming this hypothesis should be a focus of further study. Second, the equilibrium impregnated solution contents with solution $\mathrm{A}$ and $\mathrm{B}$ were two to three times as large as that with distilled water under a relative humidity of $60 \%$. This means that the adopted ALA with solution A or B has a high solution retention capability. However, differences between desorption isotherm diagrams of distilled water, solution A and solution B are not significant above RH $80 \%$ in Fig. 4. This implies that intrinsic microstructures in the adopted ALA dominates desorption properties at relative humidity above $80 \%$ and impregnated solution controls those under $80 \%$. Detailed mechanisms for this trend are not clarified in this study but are remained to investigate for later study. It should be noted that equilibrium impregnated solution contents of $\mathrm{A}$ and $\mathrm{B}$ at relative humidity of $0 \%$ were smaller than that of solid content of the solution. This implied that the ultrafine particles in the solution stayed at the near-surface zone of the ALA due to a filtering effect of ALA that is rich in independent pores.

The result of solution diffusivity is shown in Fig. 5

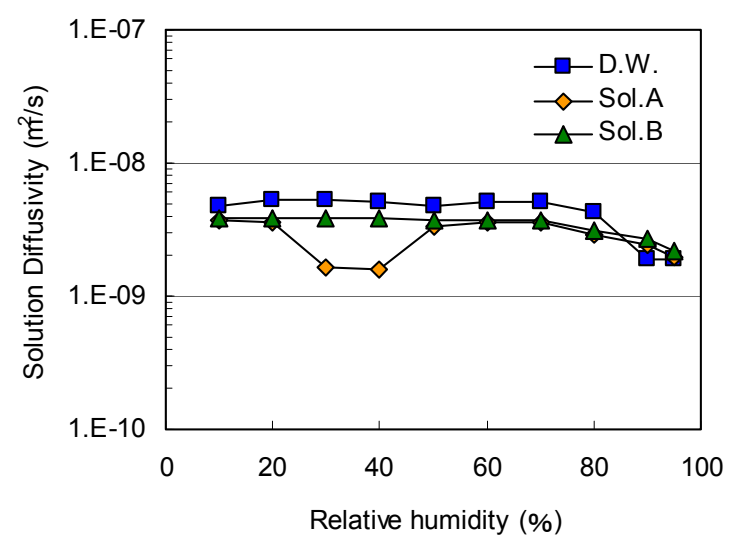

Fig. 5 Relationship between solution diffusivity and relative humidity. where it is seen that the solution diffusivities of ALA were almost the same without regard to changes in relative humidity.

With these results, it was confirmed that the adopted ALA has much higher water retention capability than reference one. Moreover, ALAs impregnated with the solutions are further improved in water retention capability than that with distilled water because the equilibrium impregnated solution contents were higher and diffusivities were smaller than those of ALA with distilled water.

\section{Highly durable concrete}

\subsection{Experimental design}

Types of concretes with smart ALAs and their constituents are shown in Table 3. Five types of concrete with a common water to cement ratio of 0.5 and a fine aggregate (natural sand) content and with different coarse aggregate types were prepared: natural aggregate type (NA), ALA with distilled water (LW, corresponding to aggregate DW in Table 1), ALAs with impregnated solution (LS, corresponding to aggregate Solution A in Table 1) and with viscosity-controlled impregnated solution (LSV, corresponding to aggregate Solution B in Table 1) and LS with a fine aggregate 10-percent substituted with fly-ash (LFS). Mix proportions of the concretes are shown in Table 4. Unit cement content, unit water content and type and unit content of fine aggregate excluding LSF were fixed while coarse aggregate type was varied using the ALAs.

Mixing of concrete was performed with a pan type mixer with a capacity of 100 liters. Materials were introduced to the mixer at one time and mixed for $90 \mathrm{sec}-$ onds. Slump and air content of each mix was $20 \pm 1.5 \mathrm{~cm}$ and $4.5 \pm 1.0 \%$ respectively showing no particular difference by mix.

Table 4 Mix proportions of concretes.

\begin{tabular}{|c|c|c|c|c|c|c|c|c|}
\hline \multirow{2}{*}{ Type } & \multirow{2}{*}{$\begin{array}{l}\text { W/B } \\
(\%)\end{array}$} & \multirow{2}{*}{$\begin{array}{l}\text { Air } \\
(\%)\end{array}$} & \multirow{2}{*}{$\begin{array}{l}\mathrm{s} / \mathrm{a} \\
(\%)\end{array}$} & \multicolumn{5}{|c|}{ Weight $\left(\mathrm{kg} / \mathrm{m}^{3}\right)$} \\
\hline & & & & $\mathrm{W}$ & $\mathrm{C}$ & $\mathrm{S}$ & FA & $\mathrm{G}$ \\
\hline NA & \multirow{5}{*}{50} & \multirow{5}{*}{4.5} & \multirow{5}{*}{47} & \multirow{5}{*}{175} & \multirow{5}{*}{350} & \multirow{4}{*}{821} & \multirow{4}{*}{-} & 943 \\
\hline LW & & & & & & & & \multirow{4}{*}{543} \\
\hline LS & & & & & & & & \\
\hline LSV & & & & & & & & \\
\hline LSF & & & & & & 739 & 69 & \\
\hline
\end{tabular}

Table 3 Experimental parameters of concretes.

\begin{tabular}{|c|c|c|c|c|c|c|}
\hline \multirow{2}{*}{ Type } & \multirow{2}{*}{ Types of Concrete } & \multirow{2}{*}{$\begin{array}{l}\mathrm{W} / \mathrm{C} \\
(\%)\end{array}$} & \multicolumn{2}{|c|}{ Aggregate types } & \multirow{2}{*}{ Impregnation solutions } & \multirow{2}{*}{$\begin{array}{l}\text { Fly ash } \\
(\%)\end{array}$} \\
\hline & & & $\mathrm{S}$ & G & & \\
\hline NA & Normal concrete & \multirow{5}{*}{50} & \multirow{5}{*}{$\begin{array}{c}\text { Natural } \\
\text { aggregates }\end{array}$} & Natural aggregates & - & - \\
\hline LW & Lightweight concrete & & & \multirow{4}{*}{$\begin{array}{c}\text { Artificial light-weight } \\
\text { aggregate }\end{array}$} & Distilled water & - \\
\hline LS & \multirow{3}{*}{ High-durability concrete } & & & & Colloidal silica & - \\
\hline LSV & & & & & Controlled Colloidal silica & - \\
\hline LSF & & & & & Colloidal silica & 10 \\
\hline
\end{tabular}




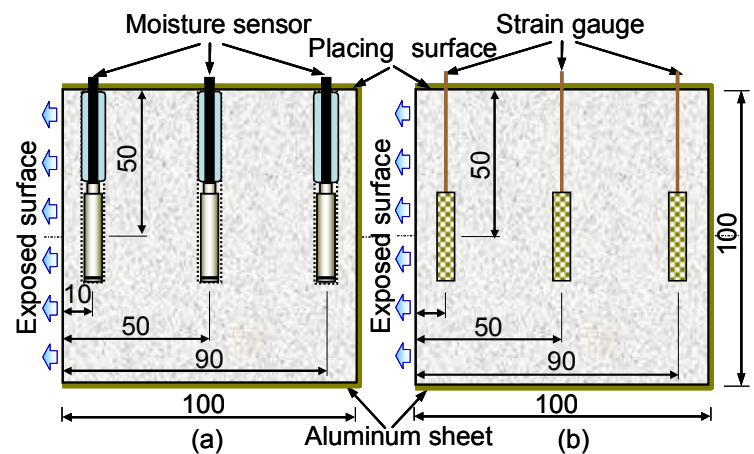

Fig. 6 Specimens for relative humidity(a) and drying-strain(b).

\subsection{Testing procedure}

\section{(1) Humidity and strain measurement}

Dimensions of the specimen and curing conditions are shown in Table 5. Specimens for relative humidity and drying strain measurement have a dimension of 100x100x100 mm where sensors were installed as shown in Fig. 6. The relative humidity inside of the concrete specimen were measured with a polymer film type small humidity sensor with a diameter of $6 \mathrm{~mm}$ inserted in holes with a depth of 10, 50 and $90 \mathrm{~mm}$ from the open surface, which has RH measuring precision of $\pm 3.0 \%$. Drying strain was measured with a two-wire, single axis type mould gauge with a length of $30 \mathrm{~mm}$ embedded at the same distance from the open surface as that of the humidity sensors.

Until the age of one week, specimens were subjected to sealed curing at a temperature of $20^{\circ} \mathrm{C}$. They were then sealed with aluminum tape leaving only one surface opened for drying and subjected to drying under relative humidity of $60 \%$ and a temperature of $20^{\circ} \mathrm{C}$.

\section{(2) Microstructure and micro-crack measure- ment}

Specimens for the microstructure and micro-cracks determination were prepared with the same dimensions and curing procedure as that for the humidity and strain measurement. Specimen preparation procedure for SEM observation is as follows.

1: At the age of 28 days, specimens were cut with a precision diamond cutter at positions 10,50 and 90 $\mathrm{mm}$ from the open surface where cubes of $12 \times 12 \times 12$ mm were extracted. Cutter was operated in the condition with round speed $2200 \mathrm{rpm}$ and position speed $0.1 \mathrm{~mm} / \mathrm{s}$.

2: Specimens were immersed in ethanol for 24 hours.

3: Specimens were dried using vacuum dry for 24 hours.

4: Specimens were placed in a vacuum container. The container was evacuated for $20 \mathrm{~min}$. to reach below $50 \mathrm{hPa}$. Then specimens were immersed into epoxy resin in the container for $30 \mathrm{~min}$. The atmosphere pressure in the container was subsequently recovered in $30 \mathrm{~min}$.

5: Specimens were removed from the container and cured in ambient room air at $20^{\circ} \mathrm{C}$.

6: After embedded in epoxy resin, the test surfaces were polished and subjected to carbon deposition.

The microstructure of the cement paste matrix was observed with a SEM-EDX at a magnification of 500 times. Ten test areas were randomly selected and the back-scattered electron image of $245 \times 184 \mu \mathrm{m}$ (2048x1536 pixels) was subjected to image analysis. A region of micro pore with a diameter smaller than $10 \mu \mathrm{m}$ was extracted from the binary image, and then micro-cracks were separated with EDX analysis targeting the carbon in the epoxy resin impregnated in micro-cracks. Micro-cracks defined as an area of $0.75 \mu \mathrm{m}^{2}$, length of $1.5 \mu \mathrm{m}$, width of $0.5 \mu \mathrm{m}$, aspect ratio of 3.0, circle-equivalent diameter of 0.977 and form factor of 1.7 was subjected to image analysis and the ratio of micro-crack area to the total area was calculated.

\section{(3) Gas permeability}

A gas permeability test was performed on the basis of the recommendation of RILEM TC116-PCD. Specimens were prepared with a polyvinyl chloride form of $150 \mathrm{~mm}$ in diameter and $50 \mathrm{~mm}$ in height. After demolding, two specimens were accumulated to have the same height of $100 \mathrm{~mm}$ as the other specimens. They were subjected to sealed curing till the age of 7 days at a temperature of $20^{\circ} \mathrm{C}$. Then the specimens were dried using an open surface under a relative humidity of $60 \%$ and a temperature of $20^{\circ} \mathrm{C}$. Using nitrogen gas, gas permeability was determined when the gas flow reached a steady state condition.

\section{(4) Accelerated carbonation}

Specimens of $100 \times 100 \times 400 \mathrm{~mm}$ were prepared. They were subjected to sealed curing until the age of 7 days

Table 5 Dimensions of the specimen and curing conditions.

\begin{tabular}{|c|c|c|}
\hline Test Item & Dimensions (mm) & Curing Conditions \\
\hline Relative humidity, Drying strain & \multirow{2}{*}{$100 \times 100 \times 100$} & \multirow{4}{*}{$\begin{array}{c}\text { Curing in condition of } 20^{\circ} \mathrm{C} \cdot \text { R.H. } 60 \% \\
\text { after sealed curing for a week }\end{array}$} \\
\hline Micro-pore and Micro-crack area & & \\
\hline Accelerated carbonation depth & $100 \times 100 \times 400$ & \\
\hline Permeability & $\varphi 150 \times 50$ & \\
\hline \multirow[b]{2}{*}{ Compressive strength } & $\varphi 100 \times 200$ & \multirow{2}{*}{$\begin{array}{c}\text { Water curing or } \\
\text { Curing in condition of } 20^{\circ} \mathrm{C} \cdot \text { R.H. } 60 \% \\
\text { after sealed curing for a week }\end{array}$} \\
\hline & $100 \times 100 \times 100$ & \\
\hline
\end{tabular}




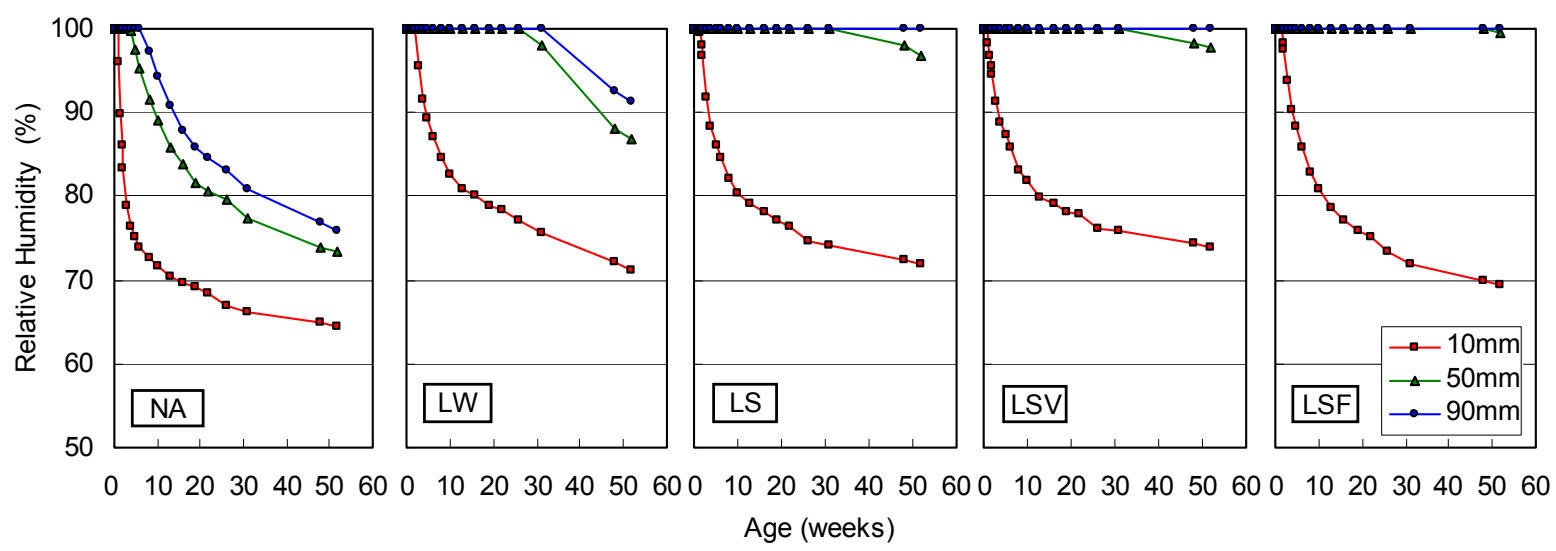

Fig. 7 Distribution of relative humidity within the specimens.

and then dried under a relative humidity of $60 \%$ and a temperature of $20^{\circ} \mathrm{C}$ till the age of 4 weeks. Subsequently, they were cured under a $\mathrm{CO}_{2}$ content of $5.0 \pm 0.2 \%$, a relative humidity of $60 \% \pm 5 \%$ and a temperature of $20^{\circ} \mathrm{C} \pm 2^{\circ} \mathrm{C}$ till the specified ages of testing.

\section{Results and discussion}

\subsection{Relative humidity and drying strain}

Distributions of relative humidity within the specimens are shown in Fig. 7. For all the specimens, relative humidity tends to decrease as it approaches to the drying surface and with time, particularly at a position $10 \mathrm{~mm}$ below the drying surface. Near-surface relative humidity of the normal concrete specimen showed abrupt decrease at one week after the start of drying and that at the center and the deepest part also started to decrease gradually after the age of 4 weeks. Relative humidity at the center and the deepest part of LW concrete kept $100 \%$ until the age of 26 weeks and then showed gradual decrease. On the other hand, relative humidity of LS, LSV, and LSF specimens kept $100 \%$ until the age of 52 weeks except for the near-surface part showing its excellent water retention capability compared to that of the normal concrete and LW.

Drying shrinkage strains in specimens are shown in Fig. 8. Upper diagram shows strains at the near-surface part, $10 \mathrm{~mm}$ from the drying surface, and lower one shows those of bottom part, $90 \mathrm{~mm}$ from the surface as shown in Fig. 6. The drying shrinkage strains at $10 \mathrm{~mm}$ from the surface increased gradually with time and, for NA concrete specimen, it developed quickly at one week after the start of drying and reached $-800 \mu$ at the age of 52 weeks, while those of LW, LSV, and LFS concretes reached around $-300 \mu$ at the age of 52 weeks. Reduction in the drying shrinkage strain was almost $500 \mu$ proving excellent water retention capability of concretes involving the adopted ALAs. Slightly larger drying shrinkage strain at $10 \mathrm{~mm}$ from drying surface for LSV and LSF than LW may be due to densified microstructure for the former two concretes. LSV and LSF have higher compressive strength as explained in a next section and ap-

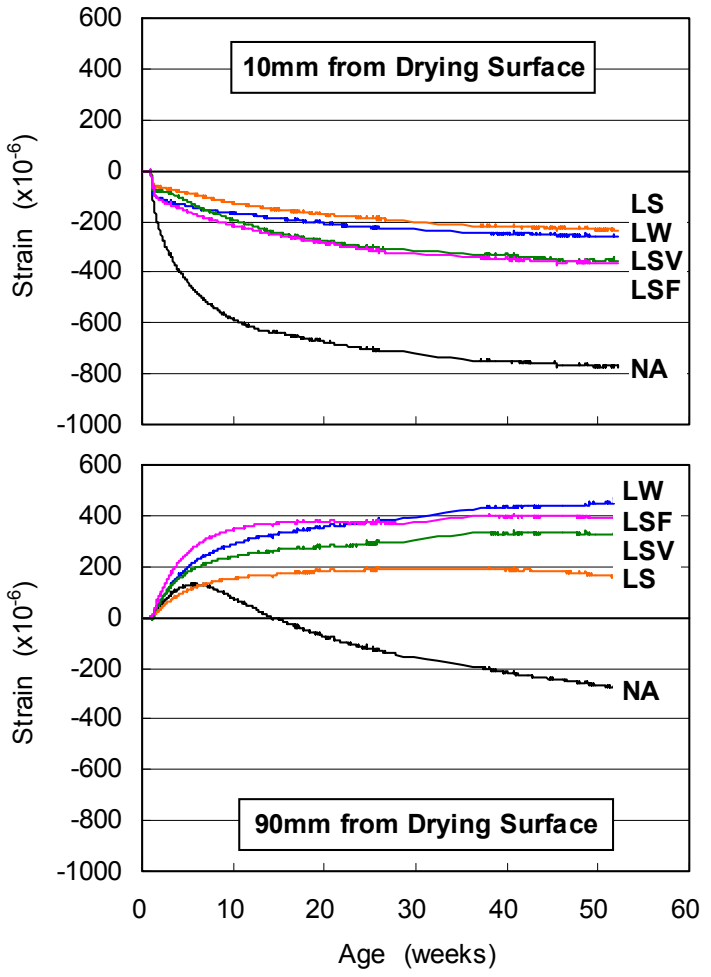

Fig. 8 Results of drying shrinkage strain.

pear to have denser microstructures and higher shrinkage coefficient, which expresses the change in strain as a function of relative humidity change (e.g., Ayano and Wittman 2002), than LW. Higher shrinkage coefficient may lead to higher drying shrinkage strain even with smaller relative humidity decrease for concretes with higher compressive strength. Strain results at $90 \mathrm{~mm}$ from the surface in Fig. 8 indicate concretes show swelling tendency except NA. This strain behavior appears to lead to very large drying shrinkage strain difference between NA and the other concretes at $10 \mathrm{~mm}$ from the surface in the upper diagram in Fig. 8. The expansive strain of LW is largest of the four concretes involving the ALAs while its internal relative humidity at $90 \mathrm{~mm}$ from the drying 
surface decreases with time away from $100 \%$. The reasons for these tendencies are not clear in this study.

Figure 9 illustrates mass decrease of specimens with time due to drying, where mass loss is larger in LW than LS, LSV, and LSF. This is considered as a clear evidence of superior water retaining capability in the proposed smart ALAs.

\subsection{Compressive strength}

Variations in compressive strength according to the curing method are shown in Fig. 10. When subjected to the standard underwater curing, compressive strengths of the cylindrical specimen generally increased with their ages while those of high-durability concretes, LS and LSV,

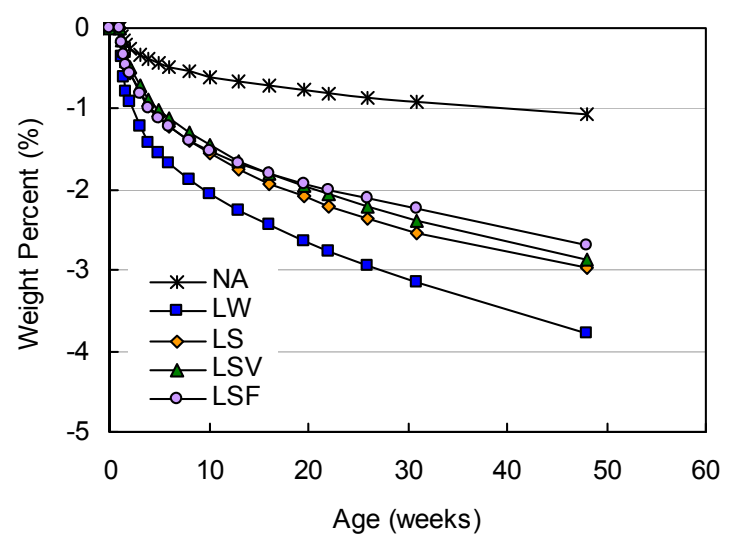

Fig. 9 Mass decreasing results of specimens due to drying.

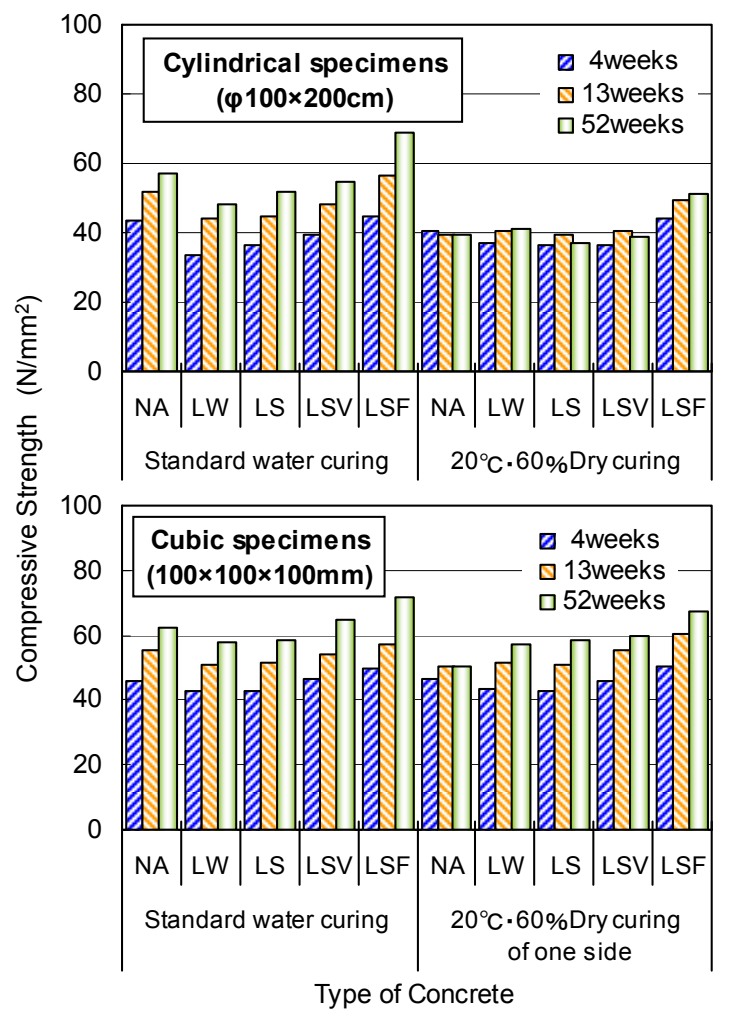

Fig. 10 Results of compressive strength. were lower than that of the normal concrete (NA) and higher than LW. The compressive strength of LSF was five to ten percent greater than that of the normal concrete. When subjected to air curing under $60 \%$ relative humidity and a temperature of $20^{\circ} \mathrm{C}$, the compressive strengths of the cylindrical specimen generally decreased with their ages except for that of LSF which became 20 percent higher than that of normal concrete. Nearly the same tendency in the variation of compressive strength was observed when cubic specimens were subjected to the standard under water curing. However when subjected to air drying, compressive strengths of high-durability concrete tended to exceed those of the normal aggregate concrete after the age of 13 weeks and much greater after 52 weeks. These results implies that the improved water retention capability and additional reactions promoted by the solutions resulted in sufficient internal curing and densification that were responsible for the development of compressive strength.

In Fig. 10, effects of internal curing on compressive strength enhancing in dry curing under $60 \%$ relative humidity and a temperature of $20^{\circ} \mathrm{C}$ are more prominent in cubic specimens than in cylinder specimens. This arises from relatively weak drying driving force in cubic specimens, which lose internal water from only one side as shown in Fig. 6. Contrary to the cubic specimens, the cylinder specimens are subjected to drying in all surfaces, which means strong drying driving force. Water retention capability due to smart ALAs appears sufficient for the conditions of the cubic specimens but not for those of the cylinder specimens. However, it should be noted that internal curing capability effective for the cubic specimens satisfies practical requirements in actual construction field. This is because the thinnest structural elements like floor slabs are subjected to similar drying driving force to the cubic specimens.

\subsection{Micro-cracks}

An example of micro-crack as imaged by the backscattered electron with a scanning electron microscope is shown in Fig. 11. Almost all the micro-cracks can be observed at the boundary of calcium silicate hydrates $(\mathrm{CSH})$ and unhydrated cement particles (UH).

Quantitative evaluation results of micro-pores and micro-cracks, which were conducted via SEM-image analysis following the condition explained in section 3.2 (2), are presented in Fig. 12. It may be a concern whether micro-cracks are additionally nucleated in the preparing procedure for SEM-observing specimens, which is specified as proc. 1 to 6 in section 3.2(2). There still exists possibility to generate additional micro-cracks before completing epoxy resin impregnating while we have preliminarily scrutinized this procedure to minimize this concern. Nucleated micro-cracks after epoxy resin impregnating are out of focus since being distinguished as inside-empty ones. For minimizing this concern, degree of micro-cracking was represented using incremental micro-crack area. Incremental micro-crack 


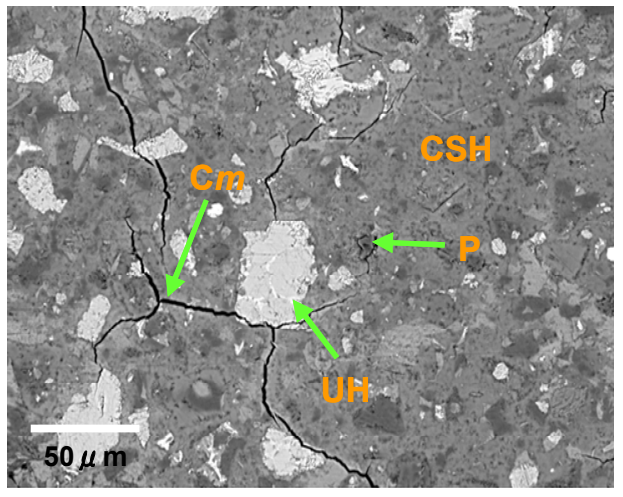

Fig. 11 Observation of micro-cracks by SEM-BEI (P:Pore, $\mathrm{Cm}$ :Microcrack,UH:Unhydrated cement particles, $\mathrm{CSH}$ : calcium silicate hydrates).

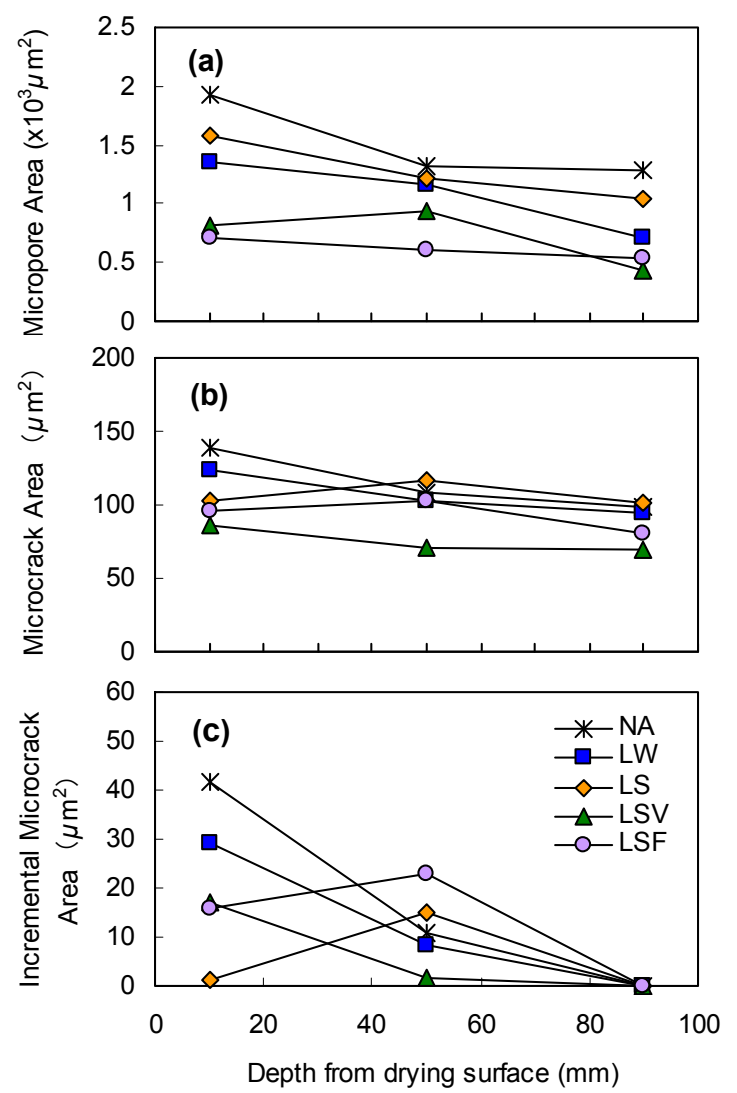

Fig. 12Results of micro-cracks and micro-pores, (a)Micropore area, (b) Microcrack area (c)Incremental Microcrack Area.

area is defined as difference of micro-crack area at $10 \mathrm{~mm}$ and $50 \mathrm{~mm}$ points from that at $90 \mathrm{~mm}$. This definition is adopted since SEM-observing specimen preparing was intended to be uniform as much as possible, and amount of the additionally nucleated micro-cracks may be similar independent of the depth in a specimen. Therefore, the incremental micro-crack area appears to reflect the effects of location in a specimen.

Figure 12 presents micro-pore area, micro-crack area and incremental micro-crack area at the age of 28 days. Fig. 12 (a) and (c) show tendency in which magnitude of micro-pore area and incremental micro-crack area are larger at near-surface than inside of the specimen. This implies that drying is a responsible driving force for micro-cracks shown in Fig. 11. It was observed that both micro-pore area and incremental micro-crack area tended to decrease in the high-durability concretes compared to those of NA and LW.

\subsection{Air permeability and carbonation depth}

Air permeability of concrete specimens is shown in Fig. 13. The air permeability tended to decrease with the age and that of the high-durability concretes was one order of magnitude smaller than that of the normal concrete implying the presence of sufficient internal curing and dense structures.

Accelerated carbonation test results are shown in Fig. 14. The carbonation depth tended to develop with time and that of the high-durability concretes was smaller than that of the normal lightweight concrete and a half of the normal concrete. This may be attributed to the favorable effects of high water retention capability and additional hydration reactions that contributed to the hydration development and densification of microstructure. Because carbonation develops inward starting at the open surface, the relationship between carbonation depth and amounts of micro-cracks and micro-pores, at the position $10 \mathrm{~mm}$ below the open surface, is shown in Fig. 15. In this figure, micro-cracks and micro-pores were represented as ratios of incremental micro-crack area and micro-pore area to total area respectively.

With above results, it was confirmed that the proposed high-durability concretes developed less micro-cracks and micro-pores resulting in the decrease in carbonation rate and depth. In this improvement, advantage of using colloidal silica solution is clear, which increases water retaining capability resulting better internal curing effects and densifies microstructure due to additional pozzolanic reaction. Micro-pore reducing effects in Fig. 12(a) and resulting durability enhancing function shown in both Fig. 14 and Fig. 15 by using controlled colloidal silica were demonstrated in experimental data while

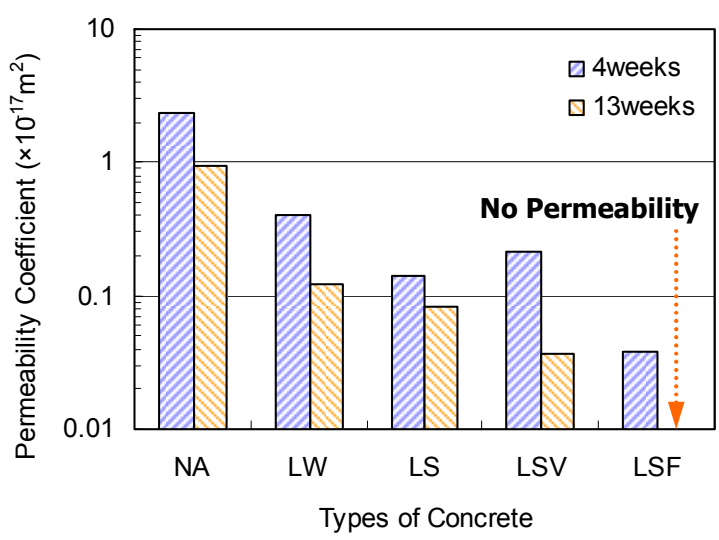

Fig. 13 Results of permeability test. 


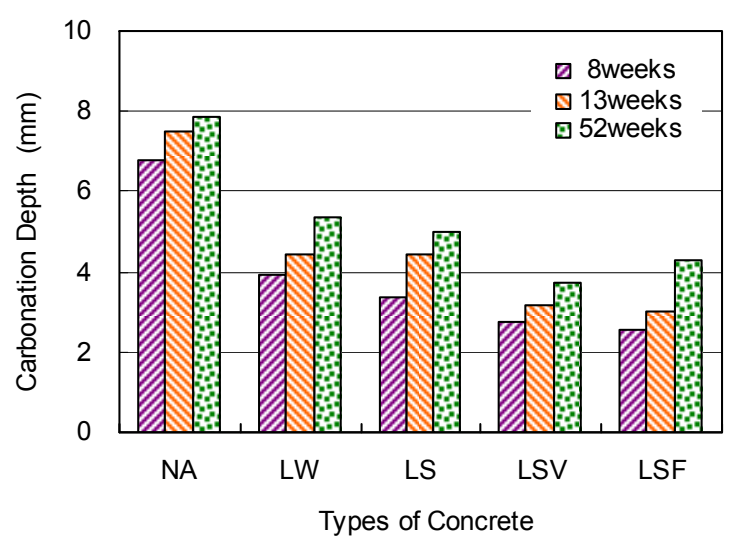

Fig. 14 Results of accelerated carbonation depth.

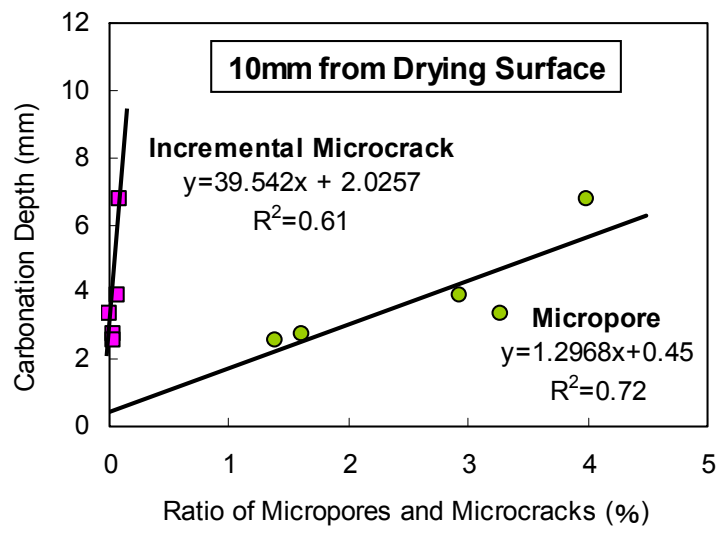

Fig. 15 Effects of micro-cracks and micro-pores on carbonation depth.

originally intended better water retaining capability than colloidal silica was not significant as shown in Fig. 9. Controlled colloidal silica may accelerate pozzolanic reaction. However, this evidence or feasible enhancing mechanism was not found in this study.

\section{Conclusions}

An artificial lightweight aggregate has been designed as a smart material and the high-durability concrete with the smart ALA was studied in terms of the development of compressive strength and the decrease in micro-cracks. The major findings are as follows.

(1) The ALA was able to incorporate the impregnated solutions as much as the maximum water content using vacuum saturation method and improvement of water retention capability was confirmed.

(2) The high-durability concrete with the smart ALA developed compressive strength more favorably than that of the normal concrete thanks to its high internal curing capability and the densified microstructure due to additional hydration reactions pro- vided by the impregnated solutions.

(3) The high-durability concrete with the smart ALA reduced the micro-cracks and micro-pores leading to an increase in durability such as decrease in carbonation and air permeability.

\section{References}

Ahn, T. and Kishi, T., (2010). "Crack self-healing behavior of cementitious composites incorporating various mineral admixtures." Journal of Advanced Concrete Technology, 8(2), 171-186.

Ayano, T. and Wittmann, F. H., (2002). "Drying, moisture distribution, and shrinkage o f cement-based materials." Materials and Structures, 35, 134-140.

Henkensiefken, R., Bentz, D., Nantung, T. and Weiss, J., (2009). "Volume change and cracking in internally cured mixtures made with saturated lightweight aggregate under sealed and unsealed conditions." Cement and Concrete Composites, 31, 427-437.

Hishiki, Y., Takada, K., Ohno, T., Ichinomiya, T., Morita, Y., (2005). "Experimental study on $150 \mathrm{MPa}$ ultra high strength concrete controlling autogenous shrinkage" Journal of Japan Society of Civil Engineers, 781, 101-112.(in Japanese)

Jensen, O. M. and Hansen, P. F., (2001). “Autogenous deformation and RH-change in perspective." Cement and Concrete Composites, 31, 1859-1865.

Jensen, O. M. and Lura, P., (2006). "Techniques and materials for internal water curing of concrete." Materials and Structures, 39, 817-825.

Ji, T. (2005). "Preliminary study on the water permeability and microstructure of concrete incorporating nano- $\mathrm{SiO}_{2}$." Cement and Concrete Research, 35, 1943-1947.

Kasai, H., Jeon, J. H., Wami, H., Fujiki, E., Tanaka, K. and Saito, A., (2008). "Development of artificial high strength light weight aggregate made from coal ash and expanding shale powder." Journal of Structures and Construction Engineering, AIJ, 73(631), 1425-1432. (in Japanese)

Tsuchiya, N., Hayano, H., Iikura, H., Kanematsu, M., Maruyama, I., Nagai, H. and Noguchi, T., (2010). "Water behavior in light-weight aggregate concrete under dying condition by neutron radiography part2: distribution of amount of water vaporization in concrete." Synopsis of annual meeting, AIJ, A-1, 1203-1204. (in Japanese)

RILEM, (2007). "Report 41: Internal Curing of Concrete - State-of-the-Art Report of RILEM Technical Committee 196-ICC," Jensen O.M. ed., 2007.

Tada, S. and Watanabe, K., (2005). "Dynamic determination of sorption isotherm of cement based materials." Cement and Concrete Research, 35, 2271-2277. 


\title{
Experimental Investigation on Reaction Rate and Self-healing Ability in Fly Ash Blended Cement Mixtures
}

\author{
Seung Hyun $\mathrm{Na}^{1}$, Yukio Hama ${ }^{2}$, Madoka Taniguchi ${ }^{3}$, Osamu Katsura ${ }^{4}$, Takahiro Sagawa ${ }^{5}$ and \\ Mohamed Zakaria ${ }^{6}$
}

\begin{abstract}
This paper investigates the hydration rate in fly ash blended cement paste and self-healing ability in mortar incorporating fly ash for long-term period. The hydration rate of fly ash and consumption of calcium hydroxide in fly ash paste containing calcium hydroxide reagents were examined at different ages and curing temperatures. Five types of fly ash blended cement paste, each of which with $10 \%$ by mass fly ash replacement ratio, were prepared for the acceleration test at $80^{\circ} \mathrm{C}$. Four fly ash cement pastes, with two types of fly ash and with fly ash replacement ratios by mass $10 \%$ and $30 \%$, were tested to measure the rate of reaction of fly ash in the mixtures. Ten mixtures were tested to evaluate the self-healing ability of mortar incorporating fly ash, considering different types of cement and fly ash. Compressive strength, bending strength, accelerated carbonation depth, after applying freeze/thaw cycling until $60 \%$ and $80 \%$ relative dynamic modulus of elasticity, and porosity were examined. The experimental results revealed that incorporating fly ash in cement paste would affect the hydration rate of fly ash and consumption of calcium hydroxide. Curing temperature can affect hydration rate, acceleration rate and velocity of reaction rate in fly ash cement paste. Moreover, it is confirmed that self-healing ability, carbonation rate coefficient and the pore volume modification in mortar incorporating fly ash rely on the curing conditions. Finally, it is suggested that the practical fly ash replacement ratio in concrete could be $10 \%$ to $15 \%$ and $11 \%$ to $20 \%$ for water to cement ratios 0.50 to 0.55 and 0.55 to 0.60 , respectively.
\end{abstract}

\section{Introduction}

Durability of concrete would be adversely degraded by micro cracks due to freezing/thawing cycles and drying/wetting cycles. Hence, it is necessary to repair appropriately the cracks in order to extend the service life of concrete structures. On the other hand, it has been well known that concrete has originally self-healing function due to rehydration of cementitious materials such as anhydrous cement in the microstructure. Self-healing of concrete is a phenomenon that micro cracks in a concrete structure naturally close under an environment with

${ }^{1} \mathrm{Ph} . \mathrm{D}$. Candidate, Graduate School of Engineering, Muroran Institute of Technology, Hokkaido, Japan. ${ }^{2}$ Professor, Graduate School of Engineering, Muroran Institute of Technology, Hokkaido, Japan.

E-Mail: hama@mmm.muroran-it.ac.jp

${ }^{3}$ Researcher, Environmental Engineering Division, Northern Regional Building Research Institute, Hokkaido Research Organization, Hokkaido, Japan.

${ }^{4}$ Senior Research Manager, Environmental Engineering Division, Northern Regional Building Research Institute, Hokkaido Research Organization, Hokkaido, Japan.

${ }^{5}$ Group leader, Nittetsu Cement Co. Ltd., Product Development Department, Muroran, Hokkaido, Japan.

${ }^{6}$ Post-Doctoral Fellow, Graduate School of Engineering, Muroran Institute of Technology, Japan, Assistant Professor, Dept. of Civil Engineering, Aswan Faculty of Engineering, Aswan University, Aswan, Egypt. water supply. To restore the original performance of damaged concrete, self-healing ability is quite needed since it is a relatively cost-effective solution compared to conventional repair methods. Fly ash, which has been increasingly used as a concrete admixture in last decades, has such kind of appropriate self-healing ability in the presence of calcium hydroxide. The filling of micro cracks, with hydrated products of fly ash and cement, can decrease the transfer of substances into damaged concrete, and hence, longer life span of concrete structures can be achieved appropriately.

In 2009, the Japan Concrete Institute committee has reported the definition of self-healing such as autogenous healing, natural healing, autonomic healing, engineered healing and activated repairing (JCI 2009). It was concluded that in the case of low water to cement ratio and high fly ash replacement ratio, the additional hydration would be caused easily by water supply around the micro cracks because of the pozzolanic reaction of fly ash. This pozzolanic reaction produces $\mathrm{C}-\mathrm{S}-\mathrm{H}$ gel that may heal micro cracks and result in better performance of cement mixtures. However, it is not sufficient to understand the self-healing mechanism in long-term period, to explain the hydration process of fly ash blended cement mixtures.

There are many studies in the literatures that have dealt with hydration property of cement based on X-ray diffraction (XRD)-Reitveld analysis. They showed that XRD-Reitveld analysis is able to investigate the hydration characteristics of cement, considering the quantitative technique, curing condition and particle size distri- 
bution in cement and amorphous admixtures (Yamaguchi et al. 1960; Asaga et al. 1992; Itoh et al. 2002; Whitfield and Mitchell 2003; Sagawa et al. 2004; Scrivener et al. 2004; Hoshino et al. 2005). Additionally, the reaction model of cement paste has been developed by Sagawa et al (2005); this model considers the thickness of hydrated products.

Sakei et al. (1997, 2004) performed a fundamental study on the reaction between fly ash and calcium hydroxide to measure the calcium hydroxide and hydration water using thermogravimetry and differential thermal analysis (TG-DTA). To calculate the reaction rate and unhydrated products of fly ash, the selective dissolution method has been reported (Ohsawa et al. 1999; Matsui and Ikabata 1999; Yamamoto and Kanazu 2004; Termkhajornkit et al. 2005).

Based on the above literatures, there is lack of information about long-term behavior of hydration process, hydrated products and self-healing ability in fly ash blended cement mixtures for different types of fly ash and cement. The objective of this study is to investigate the hydration process of fly ash and consumption of calcium hydroxide and self-healing performance in fly ash cement mixtures for long-term examination period, taking into consideration effect of cement types, fly ash types, fly ash replacement ratio and curing conditions. The observation of hydration rate of fly ash and calcium hydroxide was conducted by the combination of selective dissolution and XRD-Rietveld analysis. Thermogravimetry and differential thermal analysis (TG-DTA) was applied to quantify amount of calcium hydroxide in hardened paste. In order to introduce the micro cracks in sample, the freeze/thaw cycling test was applied to pose damage in mortar incorporating fly ash, and then, self-healing effect on accelerated carbonation tests, compressive strength, bending strength, relative dynamic modulus (RDM) of elasticity, point count test and modification of pore volume were evaluated. The porosity and pore structure modification were measured by mercury intrusion porosimetry (MIP). Finally, the obtained results from this study are valuable information for practical design codes in which the understanding of self-healing ability of fly ash is quite needed to prolong the service life of concrete structures.

\section{Experiment}

The experimental work in this study consists of three parts. The first part deals with reaction rate of fly ash and consumption of calcium hydroxide. The second part concerns with reaction rate of fly ash cement paste based on acceleration test at $80^{\circ} \mathrm{C}$, and the velocity of fly ash reaction rate. While the last part of this study concerns with self-healing effect in mortars incorporating fly ash, which exposed damage by freezing/thawing cycles, based on mechanical tests such as compressive and bending strengths, relative dynamic modulus of elasticity, accelerated carbonation test, point count test and porosity. Details of the experimental plan and investigated fly ash blended cement samples are given below.

\subsection{Reaction of fly ash and calcium hydroxide reagents}

The physical properties and chemical composition of ordinary Portland cement (OPC) and fly ash are shown in Tables 1 and 2; mineral composition and glass phase for all investigated types of fly ash are shown in Table 3 . The experimental plan for fly ash samples $4 \mathrm{~A}$ and $2 \mathrm{~B}$ is shown in Table 4. The materials were mixed 2 minutes and then casted in cubic mould with dimension of $50 \times 50 \times 50 \mathrm{~mm}$. After casting, $4 \mathrm{~A}$ and $2 \mathrm{~B}$ samples were cured in water at $60^{\circ} \mathrm{C}$ and $40^{\circ} \mathrm{C}$, respectively, for 1 day. After 1 day, they were demoulded and then moved into water till the planned ages at $60^{\circ} \mathrm{C}$ and $40^{\circ} \mathrm{C}$ for $4 \mathrm{~A}$ and $2 \mathrm{~B}$ samples, respectively. At the required ages, samples were soaked in acetone to stop the hydration, dried at $105^{\circ} \mathrm{C}$, and then ground by ball mill. The amount of unhydrated fly ash was estimated based on the selective dissolution method (Ohsawa et al. 1999; Matsui and Ikabata 1999; Yamamoto and Kanazu 2004; Termkhajornkit et al. 2005).

The rate of hydration of cement minerals was calculated by the results obtained by the XRD-Rietveld analysis. The XRD-Rietveld analysis was performed

Table 1 Characteristics of cement.

\begin{tabular}{|c|c|c|c|c|c|c|c|c|c|c|c|c|}
\hline \multirow{2}{*}{$\begin{array}{l}\text { Types of } \\
\text { cement }\end{array}$} & \multirow{2}{*}{$\begin{array}{l}\text { Density } \\
\left(\mathrm{g} / \mathrm{cm}^{3}\right)\end{array}$} & \multirow{2}{*}{$\begin{array}{c}\text { Blain } \\
\left(\mathrm{cm}^{2} / \mathrm{g}\right)\end{array}$} & \multicolumn{6}{|c|}{ Chemical composition( $\%$ ) } & \multicolumn{4}{|c|}{ Mineral composition (\%) } \\
\hline & & & $\mathrm{SiO}_{2}$ & $\mathrm{Al}_{2} \mathrm{O}_{3}$ & $\mathrm{Fe}_{2} \mathrm{O}_{3}$ & $\mathrm{CaO}$ & $\mathrm{MgO}$ & $\mathrm{SO}_{3}$ & $\mathrm{C}_{3} \mathrm{~S}$ & $\mathrm{C}_{2} \mathrm{~S}$ & $\mathrm{C}_{3} \mathrm{~A}$ & $\mathrm{C}_{4} \mathrm{AF}$ \\
\hline $\mathrm{N}$ & 3.16 & 3250 & 21.5 & 5.4 & 2.9 & 64.3 & 1.9 & 1.8 & 52 & 23 & 10 & 9 \\
\hline $\mathrm{H}$ & 3.13 & 4650 & 20.4 & 4.8 & 2.5 & 64.4 & 2.3 & 3.2 & 62 & 12 & 9 & 7 \\
\hline $\mathrm{L}$ & 3.24 & 3330 & 25.9 & 3.2 & 3.7 & 63.1 & 0.1 & 2.4 & 27 & 54 & 2 & 11 \\
\hline
\end{tabular}

Table 2 Properties of fly ash used for reaction rate tests.

\begin{tabular}{|c|c|c|c|c|c|c|c|c|c|c|c|c|c|}
\hline \multirow{2}{*}{$\begin{array}{l}\text { Types of } \\
\text { Fly ash }\end{array}$} & \multirow{2}{*}{$\begin{array}{c}\text { Blain } \\
\text { fineness } \\
\left(\mathrm{cm}^{2} / \mathrm{g}\right)\end{array}$} & \multicolumn{10}{|c|}{ Chemical composition( $\%$ ) } & \multirow{2}{*}{$\begin{array}{l}\text { Density } \\
\left(\mathrm{g} / \mathrm{cm}^{3}\right)\end{array}$} & \multirow{2}{*}{$\begin{array}{l}\text { Insoluble } \\
\text { matter(\%) }\end{array}$} \\
\hline & & $\mathrm{SiO}_{2}$ & $\mathrm{Al}_{2} \mathrm{O}_{3}$ & $\mathrm{Fe}_{2} \mathrm{O}_{3}$ & $\mathrm{CaO}$ & $\mathrm{MgO}$ & $\mathrm{Na}_{2} \mathrm{O}$ & $\mathrm{K}_{2} \mathrm{O}$ & $\mathrm{SO}_{3}$ & $\mathrm{TiO}_{2}$ & $\mathrm{MnO}$ & & \\
\hline $4 \mathrm{~A}$ & 3900 & 73.1 & 16.9 & 3.0 & 1.6 & 1.2 & 0.3 & 1.0 & 0.2 & 0.7 & 0.0 & 2.11 & 93.4 \\
\hline 4B & 4120 & 59.4 & 24.9 & 6.0 & 3.2 & 1.0 & 0.6 & 1.2 & 0.1 & 1.0 & 0.1 & 2.19 & 89.1 \\
\hline $4 \mathrm{C}$ & 4060 & 52.7 & 24.2 & 12.4 & 3.2 & 1.0 & 0.4 & 1.4 & 0.4 & 0.8 & 0.1 & 2.41 & 84.4 \\
\hline $2 \mathrm{~A}$ & 3300 & 62.0 & 22.3 & 4.7 & 2.8 & 1.9 & 1.2 & 0.3 & 1.1 & 1.1 & 0.0 & 2.21 & 91.3 \\
\hline $2 B$ & 4240 & 46.9 & 23.9 & 11.0 & 2.8 & 4.1 & 1.2 & 0.2 & 3.3 & 1.2 & 0.1 & 2.39 & 87.5 \\
\hline
\end{tabular}


Table 3 Mineral composition and glass phase for fly ash determined by XRD-Rietveld analysis.

\begin{tabular}{|c|c|c|c|c|c|}
\hline Types of fly ash & Mullite & Quartz & Magnetite & Glass phase & Ignition loss(\%) \\
\hline 4A & 4.6 & 7.8 & 0.5 & 85.1 & 2.0 \\
\hline 4B & 10.0 & 6.7 & 2.4 & 78.5 & 2.5 \\
\hline 4C & 10.5 & 8.8 & 4.4 & 73.0 & 3.4 \\
\hline 2A & 11.3 & 9.2 & 0.6 & 76.3 & 2.6 \\
\hline 2B & 8.9 & 2.8 & 2.2 & 80.7 & 5.4 \\
\hline
\end{tabular}

Table 4 Experimental plan for hydration rate test.

\begin{tabular}{|c|c|c|c|c|}
\hline $\begin{array}{c}\text { Types of } \\
\text { fly ash }\end{array}$ & $\mathrm{Ca}(\mathrm{OH})_{2}:$ Fly ash & W/B (\%) & $\begin{array}{c}\text { Curing } \\
\text { Temperature }\left({ }^{\circ} \mathrm{C}\right)\end{array}$ & $\begin{array}{c}\text { Curig age } \\
\text { (days) }\end{array}$ \\
\hline 4A & $1: 1$ & 50 & 60 & $7,14,21,28$ and 56 \\
\cline { 4 - 5 } 2B & $1: 28,70$ & 40 \\
\hline
\end{tabular}

Note: $\mathrm{W} / \mathrm{B}=$ Water / (Fly ash + Calcium hydroxide reagent).

after adding $10 \%$ of $\mathrm{Al}_{2} \mathrm{O}_{3}$, as internal reference, to the sample. The sample and internal reference were blended uniformly till becoming a harmonized texture. $\mathrm{Cu} \mathrm{K \alpha}$ X-ray diffraction system was used for conducting XRD-Rietveld analysis, with $45 \mathrm{kV}$ tube electric voltage, $40 \mathrm{~mA}$ tube current, $20-70^{\circ}$ scanning range of $2 \theta$ with scan width step of $0.02^{\circ}$. The Rietveld analysis program used in this study was Rietan-2000 software developed by Izumi and Ikeda (2000). Target minerals for analysis were $\mathrm{C}_{3} \mathrm{~S}, \mathrm{C}_{2} \mathrm{~S}, \mathrm{C}_{3} \mathrm{~A}, \mathrm{C}_{4} \mathrm{AF}$, Mullite, Quartz and Magnetite. In addition, the thermogravimetry and differential thermal analysis (TG-DTA) was performed to obtain the amount of calcium hydroxide in hardened paste. An ignition loss was measured according to JIS R5202.

\subsection{Acceleration test for fly ash cement paste 2.2.1 Acceleration test of fly ash reaction}

In order to investigate the insoluble residue contents (unhydrated amount fly ash) until the final reaction in fly ash cement paste, the acceleration test at $80^{\circ} \mathrm{C}$ of five fly ash blended cement samples, which are 4A, 4B, 4C, 2A and $2 \mathrm{~B}$ in addition to $\mathrm{N}$ sample as a reference mixture, was conducted. Each sample includes different type of fly ash and with $10 \%$ by mass fly ash replacement ratio. The experimental plan is given in Table 5. All samples mixed according to JIS R5201 before they were casted into $40 \times 40 \times 160 \mathrm{~mm}$ prisms, and then cured in water at $20^{\circ} \mathrm{C}$ for 1 day. After 1 day, the samples were cut as $3 \mathrm{~mm}$ thick, and cured in water at $80^{\circ} \mathrm{C}$. At the testing age, the samples were soaked in acetone to stop the hydration reaction, dried at $105^{\circ} \mathrm{C}$ and further ground by ball mill until particles became smaller than $75 \mu \mathrm{m}$.

\subsubsection{Measurement of velocity of fly ash reac- tion rate}

Four fly ash cement paste samples, which are 4A1, 4A3, $2 \mathrm{~B} 1$ and $2 \mathrm{~B} 3$ with two types of fly ash and with fly ash replacement ratios by mass $10 \%$ and $30 \%$ in addition to $\mathrm{N}$ sample as a reference mixture, were tested to measure the reaction rate in the mixtures. The experimental plan is shown in Table 6. Moreover, the effect of different curing conditions was also considered. All samples mixed according to JIS R5201, and then cured in water at $20^{\circ} \mathrm{C}$ for 7 days. After 7 days, the samples were cut as $3 \mathrm{~mm}$ thick, and cured in water at $5^{\circ} \mathrm{C}, 20^{\circ} \mathrm{C}$ and $40^{\circ} \mathrm{C}$. At the testing age, the samples were soaked in acetone to stop

Table 5 Experimental plan for acceleration test.

\begin{tabular}{|c|c|c|c|c|c|}
\hline Designation & $\begin{array}{c}\text { Fly ash } \\
\text { (mass \%) }\end{array}$ & W/C (\%) & W/B (\%) & $\begin{array}{l}\text { Curing Tempera- } \\
\text { ture }\left({ }^{\circ} \mathrm{C}\right)\end{array}$ & Curig age (days) \\
\hline $4 \mathrm{~A}$ & \multirow{5}{*}{10} & \multirow{6}{*}{50} & \multirow{5}{*}{45} & \multirow{6}{*}{80 in water } & \multirow{6}{*}{$\begin{array}{c}5 \mathrm{hr}, 1,2,3,4,7, \\
14,28,56 \text { and } 94\end{array}$} \\
\hline $4 \mathrm{~B}$ & & & & & \\
\hline $4 \mathrm{C}$ & & & & & \\
\hline $2 \mathrm{~A}$ & & & & & \\
\hline $2 \mathrm{~B}$ & & & & & \\
\hline $\mathrm{N}$ & 0 & & - & & \\
\hline
\end{tabular}

Note: $\mathrm{W}=$ water, $\mathrm{C}=$ Cement, $\mathrm{W} / \mathrm{B}=$ Water $/$ (Fly ash + Cement $)$.

Table 6 Experimental plan for reaction rate test.

\begin{tabular}{|c|c|c|c|c|c|c|}
\hline Designation & $\begin{array}{l}\text { Types of } \\
\text { fly ash }\end{array}$ & $\begin{array}{c}\text { Fly ash } \\
\text { (mass \%) }\end{array}$ & $\mathrm{W} / \mathrm{C}(\%)$ & $\mathrm{W} / \mathrm{B}(\%)$ & Curing Temperature $\left({ }^{\circ} \mathrm{C}\right)$ & Curig age (days) \\
\hline 4A1 & \multirow{2}{*}{$4 \mathrm{~A}$} & 10 & \multirow{5}{*}{50} & 45.5 & \multirow{5}{*}{$\begin{array}{l}5,20 \text { and } 40 \\
\text { in water }\end{array}$} & \multirow{5}{*}{$\begin{array}{c}14,21,35,63 \\
\text { and } 198\end{array}$} \\
\hline 4A3 & & 30 & & 38.5 & & \\
\hline 2B1 & \multirow{2}{*}{$2 \mathrm{~B}$} & 10 & & 45.5 & & \\
\hline 2B3 & & 30 & & 38.5 & & \\
\hline $\mathrm{N}$ & - & 0 & & 0 & & \\
\hline
\end{tabular}

Note: $\mathrm{W} / \mathrm{B}=$ Water $/$ (Fly ash + Cement). 
the hydration reaction, dried at $105^{\circ} \mathrm{C}$ and further ground by ball mill until $75 \mu \mathrm{m}$. The amount of unhydrated fly ash was estimated based on the selective dissolution method, and the rate of hydration of cement minerals was calculated by the results obtained by the XRD-Rietveld analysis; both methods apply the same procedures explained in section 2.1. TG-DTA was conducted to observe the amount of the calcium hydroxide and calcium carbonate in hardened paste (Ohsawa et al. 1999). An ignition loss was tested according to JIS R5202.

\subsection{Self-healing of fly ash}

Ten mixtures were tested to evaluate the self-healing ability of mortar incorporating fly ash, considering different types of cement, fly ash, fly ash replacement ratio and curing conditions. The experiments used ordinary Portland cement $(\mathrm{N})$, high early strength Portland cement $(\mathrm{H})$ and low heat Portland cement (L). The fly ash characteristic properties, such as blain fineness, ignition loss and glass phase, were given in Table 7. The mortar mixtures were prepared with the proportions summarized in Table 8. It is well known that the reaction rate of fly ash is late, in fact that may cause a decrease of the compressive strength at early age. This drawback can be improved by using high early strength Portland cement type, therefore, the high early strength Portland cement type $(\mathrm{H})$ was chosen in order to avoid the decrease of compressive strength at early ages. In addition, low heat Portland cement has a large amount of $\mathrm{C}_{2} \mathrm{~S}$ (belite), unlike ordinary Potland cement and that may improve the long-term reaction. The physical properties and chemical composition of ordinary Portland cement $(\mathrm{N})$, high early strength Portland cement $(\mathrm{H})$ and low heat

Table 7 Properties of the fly ash used for self-healing ability tests.

\begin{tabular}{|c|c|c|c|}
\hline $\begin{array}{c}\text { Types of } \\
\text { fly ash }\end{array}$ & $\begin{array}{c}\text { Blain fineness } \\
\left(\mathrm{cm}^{2} / \mathrm{g}\right)\end{array}$ & $\begin{array}{c}\text { Ignition loss } \\
(\%)\end{array}$ & $\begin{array}{c}\text { Glass Phase } \\
(\%)\end{array}$ \\
\hline A & 4090 & 1.5 & 88.8 \\
\hline B & 4040 & 2.1 & 86.2 \\
\hline C & 3160 & 1.9 & 88.4 \\
\hline D & 2830 & 1.7 & 80.6 \\
\hline
\end{tabular}

Portland cement (L) are shown in Table 1. The cement replacement ratio of these two types cement could be calculated from mineral composition. The ten mortar mixtures consist of one OPC mortar sample $(\mathrm{N})$ without fly ash replacement and eight fly ash cement mortar samples with mixing ratio 7 to 3 for high early strength Portland cement to low heat Portland cement, respectively, and with fly ash replacement ratio as $10 \%, 20 \%$ and $30 \%$ by volume. The water to cement ratio (W/C) in this experiment varied as $45 \%, 55 \%$ and $65 \%$. After casting, samples were cured under a condition of $20^{\circ} \mathrm{C}$ for 4 weeks in water. In order to introduce the micro cracks in the sample, the freeze/thaw test was performed until relative dynamic modulus of elasticity (RDM) $60 \%$ and $80 \%$, in accordance with JIS A 1127 . The relative dynamic modulus of elasticity was determined by measuring the resonant frequency and was calculated as the percentage fraction of square transverse frequency after $c$ cycles of freeze/thaw test to square transverse frequency at 0 cycle of freeze/thaw test. After deterioration of samples, different curing conditions were applied as $20^{\circ} \mathrm{C}$ for 1 week and $40^{\circ} \mathrm{C}$ for 4 weeks in water. Then, tests for physical properties, which are compressive test, bending test, accelerated carbonation depth test and point count procedure, of samples at testing ages were performed.

In the point count test, which was used to quantify the change of the number of cracks after deterioration, the number of cracks in sample was analyzed using a modified point count procedure which was suggested by $T$. Matsumura et al. (2003) who investigated experimentally the relationship between the number of cracks and degree of frost damage by means of image analysing microscope (with $100 \times$ magnification). It was inferred that the decrease of relative dynamic modulus can increase the number of cracks in concrete sample after the frost damage has taken place. In the accelerated carbonation test, at condition of $20^{\circ} \mathrm{C}$ and $60 \% \mathrm{RH}$, after 4 weeks the samples were dried in advance, and then this test was carried out with $5 \% \mathrm{CO}_{2}$ concentration at 1,4 and 13 weeks. Finally, the PoreMaster 33 mercury intrusion porosimetry (MIP) was used to measure the pore

Table 8 Mortar mix proportion.

\begin{tabular}{|c|c|c|c|c|c|c|c|c|c|c|c|}
\hline \multirow{3}{*}{ Designation } & \multirow{3}{*}{$\begin{array}{l}\mathrm{W} / \mathrm{C} \\
(\%)\end{array}$} & \multirow{3}{*}{$\begin{array}{l}\text { Types } \\
\text { of } \\
\text { fly ash }\end{array}$} & \multirow{3}{*}{$\begin{array}{c}\mathrm{F} /(\mathrm{S}+\mathrm{F}) \\
(\mathrm{vol} \%)\end{array}$} & \multirow{3}{*}{$\begin{array}{c}\text { Degree of } \\
\text { deterioration } \\
(\%)\end{array}$} & \multirow{3}{*}{$\begin{array}{l}\text { Curing age and } \\
\text { temperature after } \\
\text { deterioration }\left({ }^{\circ} \mathrm{C}\right)\end{array}$} & \multicolumn{6}{|c|}{ Unit weight $\left(\mathrm{kg} / \mathrm{m}^{3}\right)$} \\
\hline & & & & & & \multirow{2}{*}{$\mathrm{W}$} & \multicolumn{3}{|c|}{ cement } & \multirow{2}{*}{$\mathrm{F}$} & \multirow{2}{*}{ S } \\
\hline & & & & & & & $\mathrm{N}$ & $\mathrm{H}$ & $\mathrm{L}$ & & \\
\hline $\mathrm{N}$ & \multirow{5}{*}{55} & - & - & \multirow{3}{*}{60 and 80} & \multirow{10}{*}{$\begin{array}{l}20 \text { at } 1 \text { week } \\
\text { and } \\
40 \text { at } 4 \text { weeks } \\
\text { in water }\end{array}$} & 342 & 621 & - & - & - & 1241 \\
\hline HL & & - & - & & & 342 & - & 434 & 187 & - & 1241 \\
\hline FA20 & & \multirow{6}{*}{ A } & 20 & & & 342 & - & 434 & 187 & 97 & 1119 \\
\hline FA10 & & & 10 & \multirow{7}{*}{$60 \%$} & & 342 & - & 434 & 187 & 195 & 994 \\
\hline FA30 & & & 30 & & & 342 & - & 434 & 187 & 293 & 870 \\
\hline FAX & 45 & & \multirow{5}{*}{20} & & & 316 & - & 492 & 211 & 221 & 1125 \\
\hline FAY & 65 & & & & & 380 & - & 409 & 175 & 183 & 937 \\
\hline FB & \multirow{3}{*}{55} & & & & & 342 & - & 434 & 187 & 195 & 994 \\
\hline FC & & $\mathrm{C}$ & & & & 342 & - & 434 & 187 & 207 & 994 \\
\hline FD & & $\mathrm{D}$ & & & & 342 & - & 434 & 187 & 205 & 994 \\
\hline
\end{tabular}

Note: $\mathrm{W}=$ water, $\mathrm{N}=$ Ordinary Portland cement, $\mathrm{H}=$ High early strength Portland cement, 
structure distribution and its modification in the mortar samples, so that the influence of fly ash and the curing condition on the change of pore structure volume in studied samples can be examined. In order to determine the hydration rate of fly ash for normal slab concrete in Asahikawa, Japan, the natural temperature and humidity were investigated for 2 years. The water to cement ratio $(\mathrm{W} / \mathrm{C}$ ) of normal concrete in this experiment was $50 \%$.

\section{Results and discussion}

\subsection{Reaction of fly ash and calcium hydroxide reagent}

The methods based on selective dissolution (Ohsawa et al. 1999; Matsui et al. 1999) can be adopted as the calculation method of reaction rate of unhydrated fly ash. The phase constitution of fly ash in both unreacted process and reaction process is considered in Fig. 1. Glass contents in the unreacted process can be calculated by deducting the amount of crystalline phase and ignition loss from unreacted fly ash amount. The glass contents can be divided into two parts which are acid soluble amount $(A s)$ and acid insoluble matter $(A u)$. As for the crystal phase part, assuming that it does not dissolve in acid, acid insoluble matter can be obtained by excluding the amount of crystal phase from the test results for the insoluble residue quantity. Thus, the calculation method of reaction ratio by selective dissolution method can be explained as follows. When fly ash reacts, it becomes the substance where portion of insoluble matter dissolves in acid. Here, selective dissolution test is carried out, and the unreacted part of acid insoluble matter is measured as an insoluble residue. Then, the dissolved contents can be estimated from reacted weights of paste. The following patterns are considered for the calculation method.

Pattern 1: it is related to the melting ratio of glass phase and crystal phase $(\mathrm{Cr})$. This pattern $(\mathrm{Rec})$ is calculated as follows:

$$
\operatorname{Rec}=(A s+A r) /(A u+A s+C r)
$$

Pattern 2: it is related to the melting ratio for glass phase. This pattern $(R g)$ is expressed as follows:

$$
R g=(A s+A r) /(A u+A s)
$$

Pattern 3: it can be estimated from the melting ratio for acid insoluble matter. This pattern ( Rus ) is given as shown in Eq. (3):

$$
R u s=A r /(A u+C r)
$$

Pattern 4: It can be obtained from the melting ratio for the acid insoluble matter in the glass phase. This pattern ( Rau ) is calculated as in Eq. (4):

$$
\operatorname{Rau}=\mathrm{Ar} / \mathrm{Au}
$$

Figure 2 shows the comparison of the consumption of calcium hydroxide contents and reaction rate of fly ash for each pattern. Relationship between the reaction rate and the calcium hydroxide consumption for fly ash cement paste can be expressed as given in Eq. (5):

$\mathrm{Ca}(\mathrm{OH})_{2}$ consumption contents $=W_{0}-\frac{W_{c c}+\left(W_{c c} \times 74.09 / 100.09\right)}{\left(100-R_{w}\right) \times 0.01}$

Where, $W_{0}$ is calcium hydroxide contents within $1 \mathrm{~g}$ of unreacted sample $(\mathrm{g} / \mathrm{g}), W_{c h}$ is the calcium hydroxide weights which include in unreacted sample $1 \mathrm{~g}(\mathrm{~g} / \mathrm{g})$, $W_{c c}$ is calcium carbonate weights in hydrated sample $1 \mathrm{~g}$ $(\mathrm{g} / \mathrm{g})$ and $R_{w}$ is the amount of bound water in $1 \mathrm{~g}$ within hydration sample $(\%)$.

By observing Figs. 2 (a) and (b), which represent patterns 1 and 2, respectively, it can be inferred that, although calcium hydroxide consumption is almost zero, the reaction rate is not identical to zero. This is due to the fact that pozzolanic reaction exists in fly ash and calcium hydroxide. However, it cannot be determined which part of fly ash that reacting with calcium hydroxide. The data plotted in Figs. 2(c) and (d), which explains behavior of patterns 3 and 4, respectively, reveals that when the consumption of calcium is almost zero, there is no contribution of calcium to reaction rate. It is obvious from the comparison given in Fig. 2 that pattern 4 gives the best response for reaction rate of fly ash compared to other patterns 1, 2 and 3. The reason for this response is due to the assumption of pattern 4 , in which the reaction rate is calculated based on the melting ratio for the acid insoluble matter in the glass phase, meaning that there is no contribution of crystalline phase $(\mathrm{Cr})$ to reaction rate. From the results above, the reaction of fly ash with calcium hydroxide can be considered, when acid insoluble

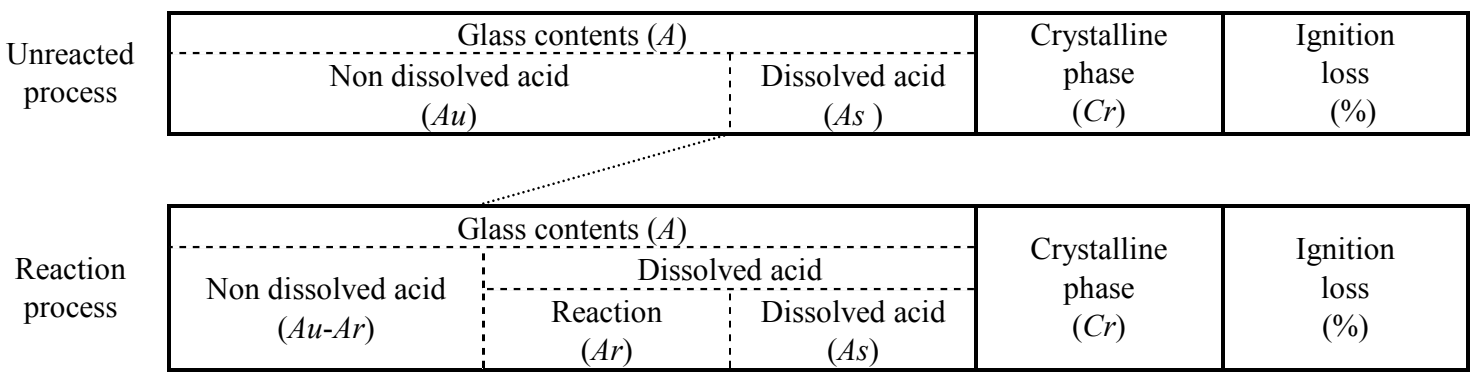

Fig. 1 Schematic reaction models. 


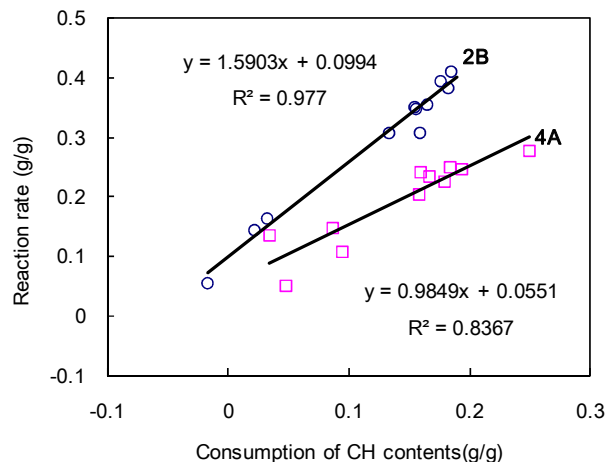

(a) Pattern 1

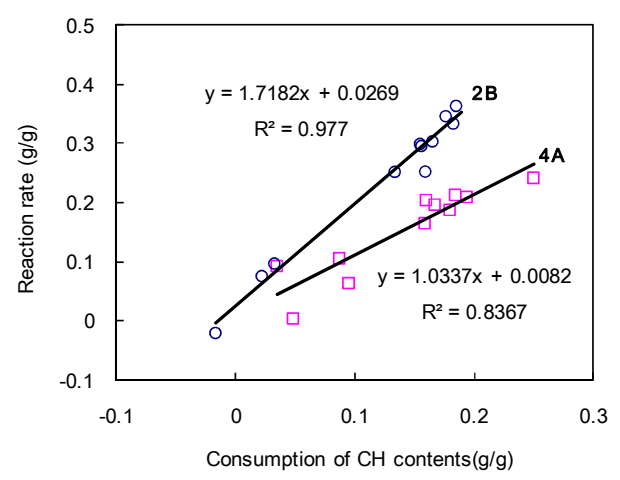

(c) Pattern 3

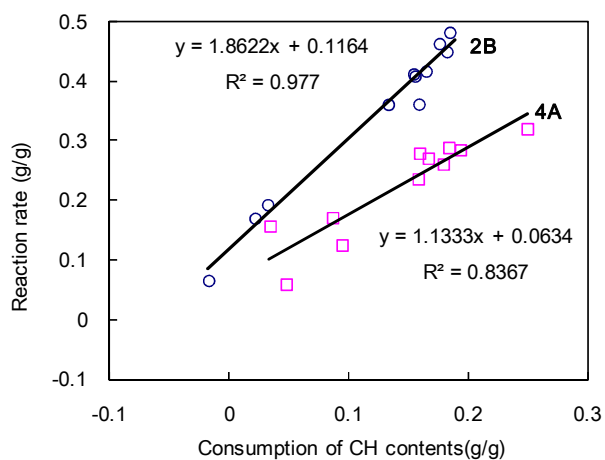

(b) Pattern 2

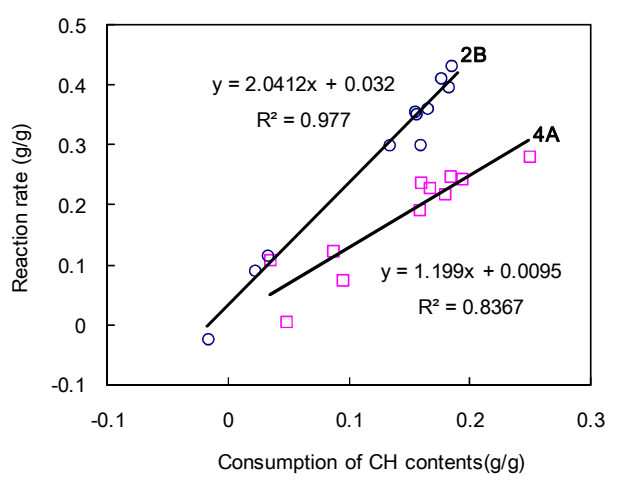

(d) Pattern 4

Fig. 2 Comparison of the consumption of $\mathrm{CH}$ contents and reaction rate of fly ash.

matter in the glass phase exists, as given by Eq. (4).

\subsection{Fly ash cement paste acceleration reaction test at $80^{\circ} \mathrm{C}$}

Figure 3 presents the comparison of the pozzolanic reaction rate and curing age (days) for 5 types of fly ash cement paste. It can be inferred from the figure that the reaction rate for sample $4 \mathrm{C}$, at early age, is higher than other samples. At 94 days, the reaction rate for sample $2 \mathrm{~A}$ is increased to $100 \%$, while samples $4 \mathrm{C}$ and $2 \mathrm{~B}$ almost have the same reaction rate. The results shown in Fig. 3 reveal that acid insoluble matter of the glass phase of fly ash (FA) reacts almost $100 \%$, for all types of fly ash in the study at 94 days. In Fig. 4, the relationship between remaining contents of calcium hydroxide and curing ages (days) is described. The amount of calcium hydroxide in the hardened paste can be calculated as shown in Eq. (6):

Calcium hydroxide contents $=\frac{W_{c h}+\left(W_{c c} \times 74.09 / 100.09\right)}{\left(100-R_{w}\right) \times 0.01}$

Where, $W_{c h}$ is the calcium hydroxide weight which includes in unreacted sample $1 \mathrm{~g}(\mathrm{~g} / \mathrm{g}), W_{c c}$ is calcium carbonate weights in hydrated sample $1 \mathrm{~g}(\mathrm{~g} / \mathrm{g})$ and $R_{w}$ is the amount of bound water in $1 \mathrm{~g}$ within hydration sample $(\%)$

In comparison with $\mathrm{N}$ sample, the remaining contents of calcium hydroxide in fly ash cement samples after 2

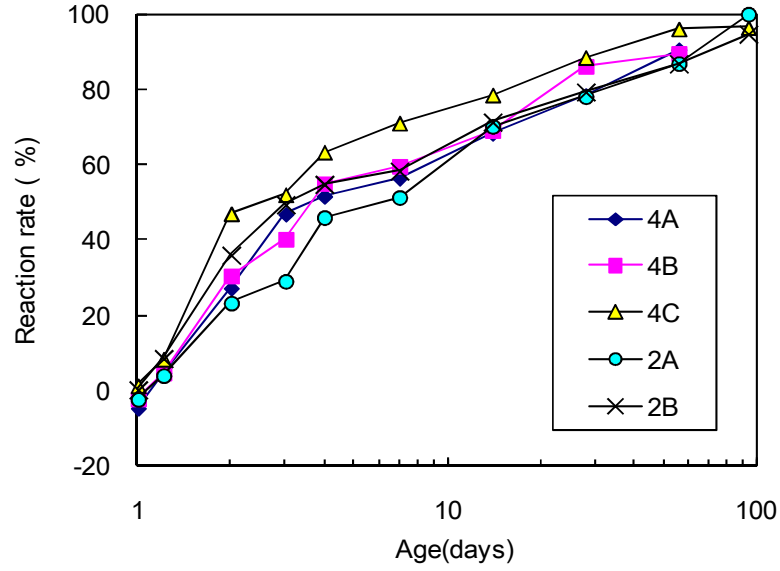

Fig. 3 Comparison of the pozzolanic reaction rate of different fly ash types versus curing age (days).

days decrease because of its pozzolanic reaction. For the remaining calcium hydroxide contents at each age, it is found that there is no significant difference among the types of fly ash. Although the pozzolanic reaction rate is almost $100 \%$, the calcium hydroxide in paste remains after 56 days. Therefore, in case of cement paste with $10 \%$ by mass fly ash replacement ratio, it can be considered that the glass phase and insoluble residue contents in fly ash could react with the calcium hydroxide. 


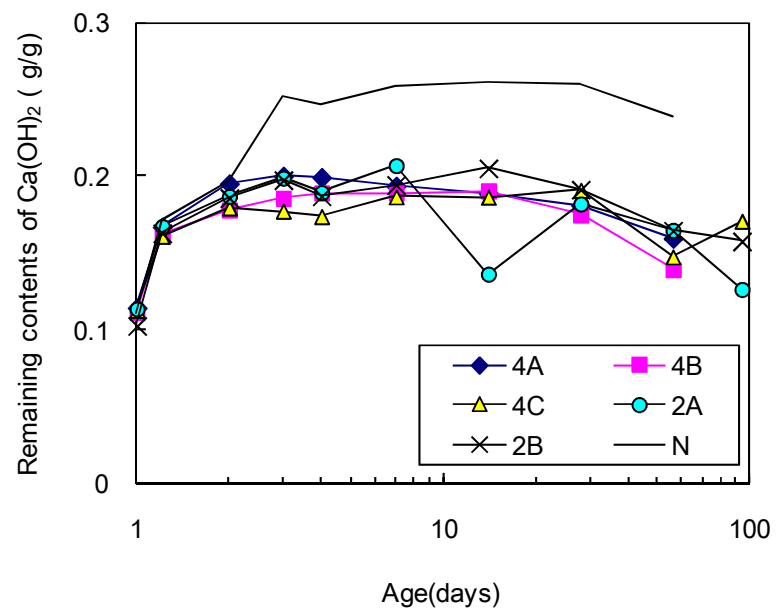

Fig. 4 Remaining contents of calcium hydroxide versus curing age (days).

\subsection{The reaction rate of fly ash cement paste 3.3.1 Amount of calcium hydroxide}

In Fig. 5, the amount of remaining calcium hydroxide in hardened fly ash paste based on the XRD-Rietveld analysis is plotted versus testing age for different curing conditions. From the figure, it can be revealed that in the case of cement paste incorporating fly ash (samples $4 \mathrm{~A}$ and $2 \mathrm{~B}$ ), the amount of remaining calcium hydroxide is lower than $\mathrm{N}$ sample which is the case of OPC paste without fly ash. Also, it is obvious that fly ash cement samples with fly ash replacement ratio by mass $30 \%$, and with high curing temperature in water (samples $4 \mathrm{~A} 3-40^{\circ} \mathrm{C}$ and $2 \mathrm{~B} 3-40^{\circ} \mathrm{C}$ ), yielded smaller remaining amount of calcium hydroxide compared to other samples with smaller fly ash replacement ratio (samples $4 \mathrm{~A} 1-40^{\circ} \mathrm{C}$ and $2 \mathrm{~B} 1-40^{\circ} \mathrm{C}$ ). This observation confirms that large amount of fly ash as $30 \%$ replacement ratio and high curing temperature in water can increase the consumption amount of calcium hydroxide. The reason for this behavior is due to the pozzolanic reaction of fly ash, which increases with increasing the amount of fly ash and curing temperature, and thus consumes more calcium hydroxide that comes from cement.

\subsubsection{Prediction equation of reaction rate}

For the hydration reaction of cement, the reaction rate for each cement minerals calculated by Sagawa et al. (2004, 2005) equation can be expressed as Eq. (7):

$$
d x / d t=k(1 / x)
$$

Where, $x$ is reaction thickness to inner particle, $k$ is a constant represent the reaction rate, and $t$ is reaction time. The hydration rate $(k)$ can be derived from the following equation:

$$
k=A \exp (-E a / R T)
$$

Where, $A$ is experimental constant, $E a$ is apparent activation energy $(\mathrm{J} / \mathrm{mol}), R$ is universal gas constant
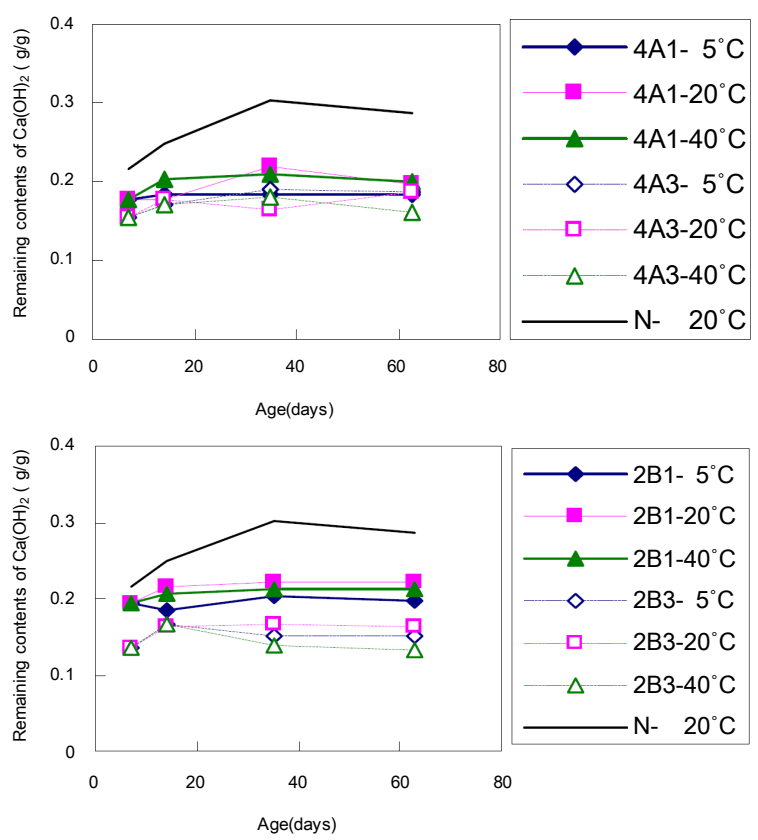

Fig. 5 Remaining contents of $\mathrm{Ca}(\mathrm{OH})_{2}$ versus curing age(days).

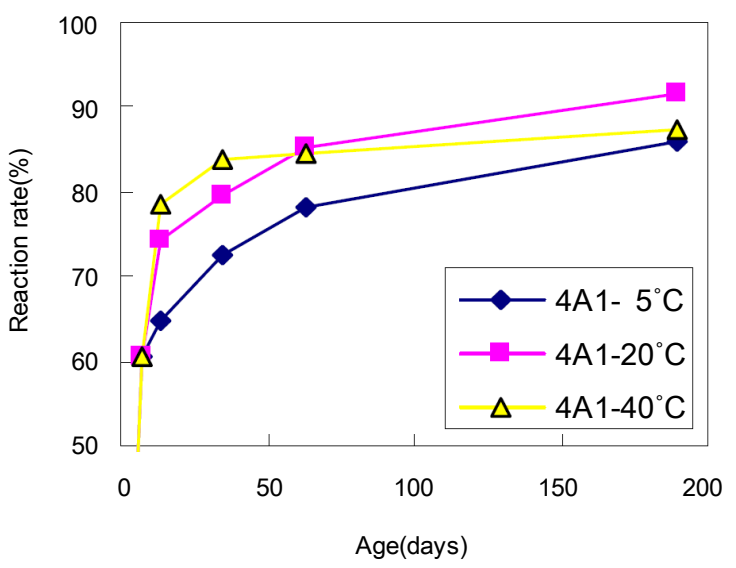

Fig. 6 Rate of reaction of cement in fly ash cement mixture.

and $T$ is temperature. In addition, an average particle size of fly ash in radius $(r)$ can be expressed as in Eq. (9):

$$
r=3 /(\rho \times s)
$$

Where, $s$ is blaine fineness of fly ash $\left(\mathrm{cm}^{2} / \mathrm{g}\right)$ and $\rho$ is the density of fly ash $\left(\mathrm{g} / \mathrm{cm}^{3}\right)$.

The above equation can applied in the case of $x \geq r$, however, if $x<r$ the following equation can be used:

$$
\operatorname{Fr}(\%)=\left(1-(r-x)^{3} / r^{3}\right) \times 100
$$

\subsubsection{Reaction of cement in fly ash blended paste}

The rate of reaction of cement for fly ash paste sample $4 \mathrm{~A} 1$ with curing temperature varies as $5^{\circ} \mathrm{C}, 20^{\circ} \mathrm{C}$ and $40^{\circ} \mathrm{C}$ is given in Fig. 6. This data is calculated by the 
unreacted mineral composite based on the XRD-Rietveld analysis. Although the cement, which was blended with fly ash, could not react fully as $100 \%$, the reaction rate is almost $85 \%$, containing $\mathrm{C}_{2} \mathrm{~S}$ and $\mathrm{C}_{4} \mathrm{AF}$. The figure shows that there is influence of the curing temperature on the cement reaction rate for different ages; this is obvious when comparing the response for reaction rate of sample $4 \mathrm{~A} 1-20{ }^{\circ} \mathrm{C}$ with sample $4 \mathrm{~A} 1-5^{\circ} \mathrm{C}$.

\subsubsection{Reaction of fly ash}

For the rate of fly ash reaction, the dissolved ratio can be estimated by the insoluble matter contents in glass phase. The apparent activation energy (Ea) can be calculated by Eq. (8) and was obtained based on a nonlinear regression analysis applied to the experimental results; its value is given in Table 9. Both two types of fly ash have the same apparent activation energy (Ea), but the experimental constant (A) is slightly different. Figure 7 shows the actual and predicted hydration rate (straight line) of fly ash in fly ash cement samples for different curing conditions and fly ash replacement ratios. In the case of $4 \mathrm{~A} 1$ and $2 \mathrm{~B} 1$ samples $(10 \%$ by mass replacement ratio of fly ash), the actual and predicted hydrations at the final reaction in paste have the same tendency for all curing condition cases. However, in the case of $4 \mathrm{~A} 3$ and $2 \mathrm{~B} 3$ samples ( $30 \%$ by mass replacement ratio of fly ash), there is no identical tendency at the final reaction for all
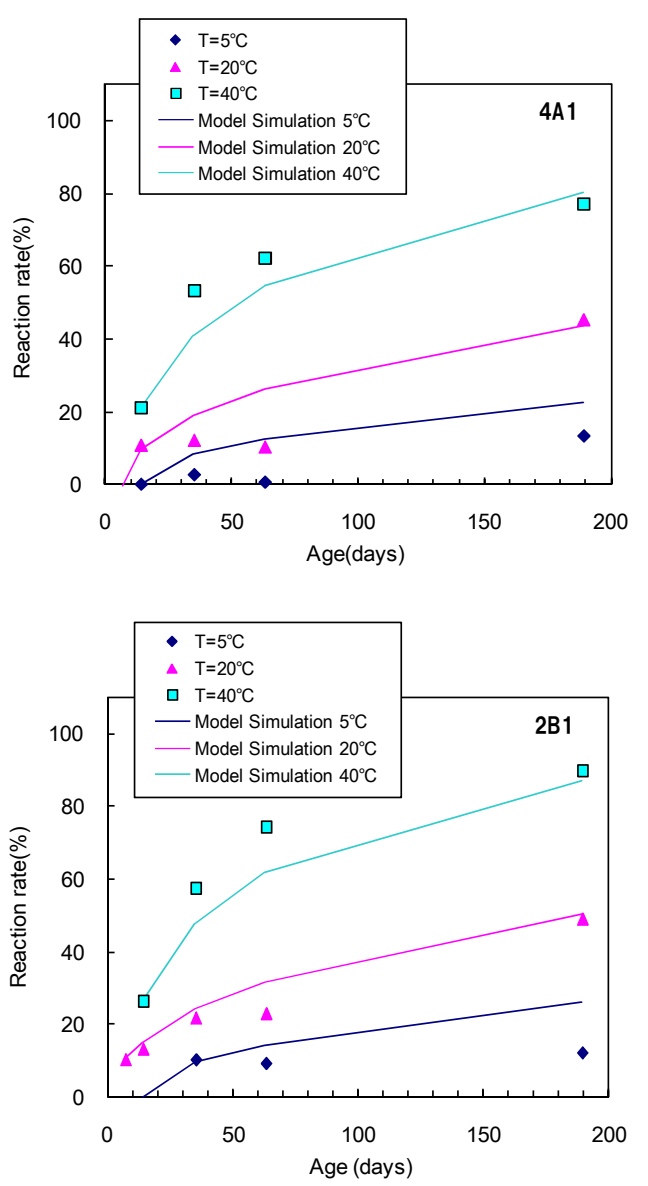

Table 9 Velocity coefficient of reaction rate.

\begin{tabular}{|c|c|c|}
\hline & $4 \mathrm{~A}$ & $2 \mathrm{~B}$ \\
\hline$E a(\mathrm{~kJ} / \mathrm{mol})$ & 67.1 & 67.1 \\
\hline$A\left(\times 10^{8}\right)$ & 10.1 & 9.2 \\
\hline
\end{tabular}

Note: $E a=$ Apparent activation energy,

$A=$ Experimental constant

curing conditions. It can be inferred from Fig. 7 that the reaction rate of fly ash depends on the curing temperature, curing age and fly ash replacement ratio.

\subsection{Self-healing effects}

Figures 8, 9 and 10 show the results of compressive strength, bending strength and relative dynamic modulus of elasticity for fly ash blended cement mortar samples with four fly ash types, replacement ratios vary as $10 \%$, $20 \%$, and $30 \%$ by volume and three types of cement. Ten mortar mixtures were prepared with the proportions summarized in Table 8 and section 2.3. The examined mortar samples consist of $\mathrm{N}$ sample using OPC without fly ash replacement, nine samples using combination of high early strength Portland cement and low heat Portland cement. It can be seen that the investigation of each mortar sample includes four cases which are no cracking case, after deterioration case, cured in water $20^{\circ} \mathrm{C}$ at
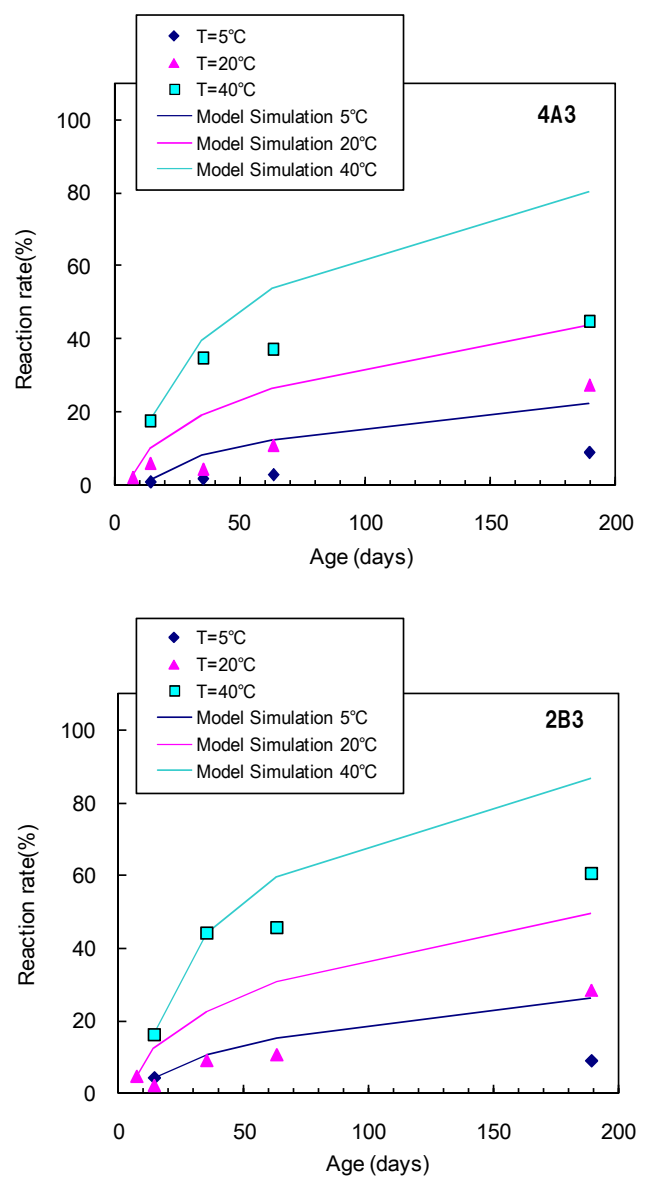

Fig. 7 Predicted results of fly ash reaction rate versus experimental values. 


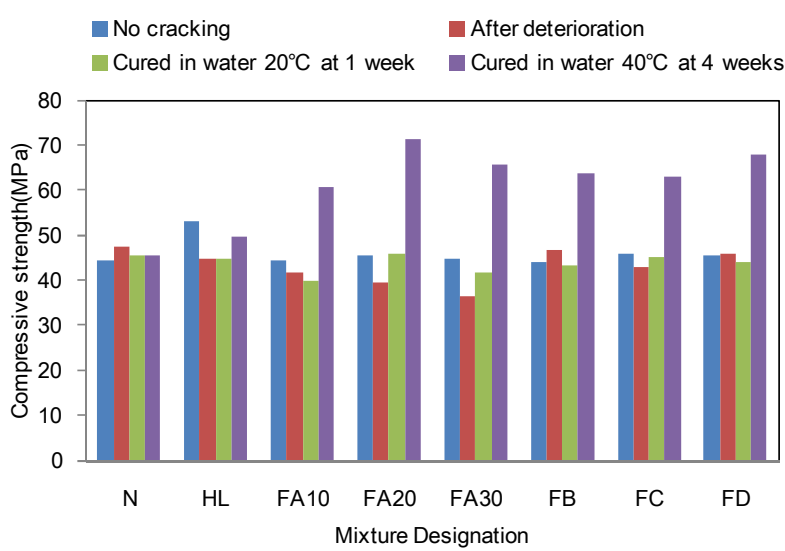

Fig. 8 Self-healing effects on compressive strength for different mixtures and curing conditions.

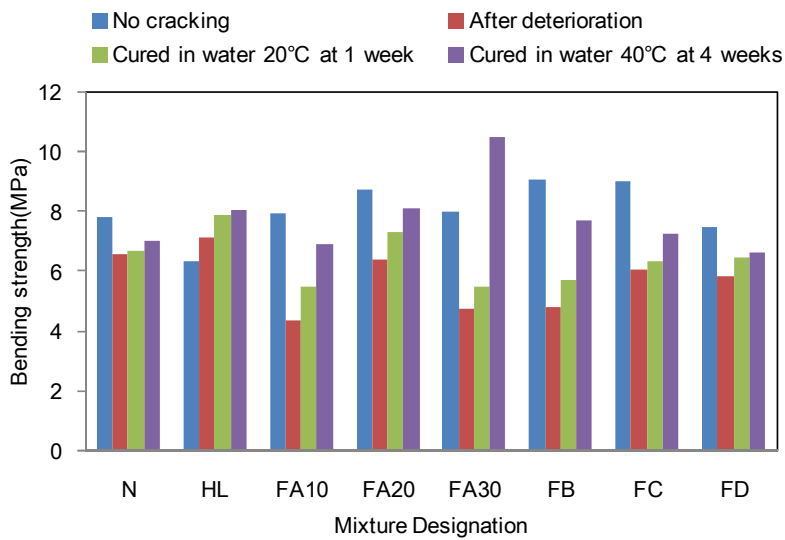

Fig. 9 Self-healing effects on bending strength for different mixtures and curing conditions.

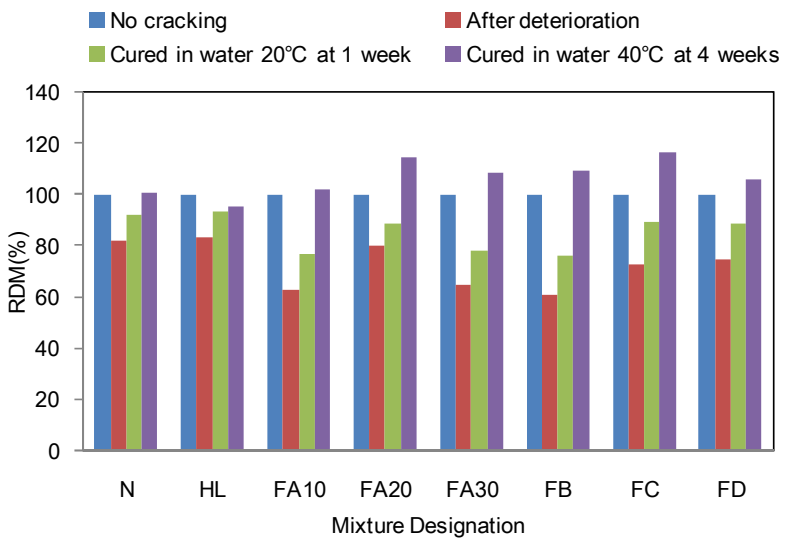

Fig. 10 Self-healing effects on relative dynamic modulus of elasticity for different mixtures and different curing conditions.

1 week case and cured in water $40^{\circ} \mathrm{C}$ at 4 weeks case; the results of each case are shown in Figs. 8 through $\mathbf{1 0}$. After deterioration, it can be seen from the figures that most of the investigated samples can heal the compressive strength, bending strength and RDM. However, degree of healing in mortar samples incorporating fly ash is higher compared to $\mathrm{N}$ sample, which is the case of

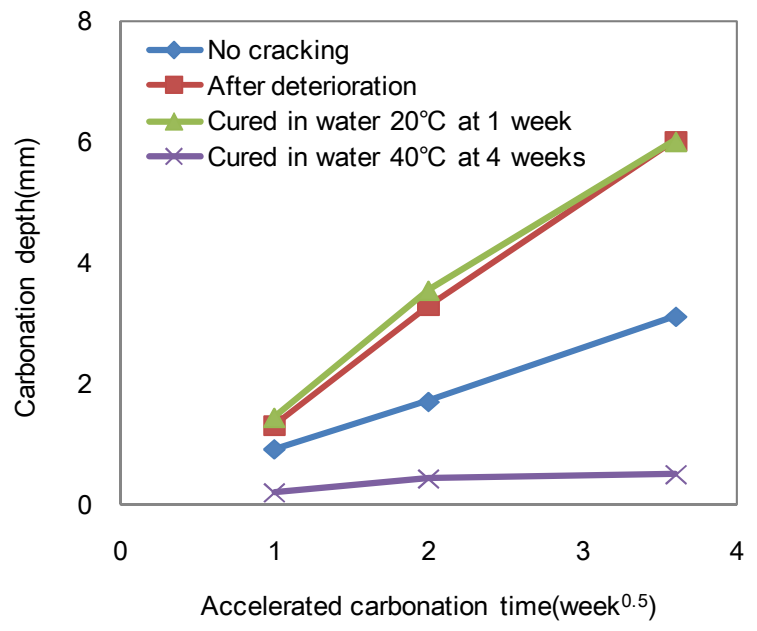

Fig. 11 Change of carbonation depth for FA30 sample.

OPC mortar without fly ash, especially for the case of curing in water $40^{\circ} \mathrm{C}$ at 4 weeks. Also, it is evident that the curing condition in water $20^{\circ} \mathrm{C}$ at 1 week is not enough to heal the deterioration compared to the curing condition in water $40^{\circ} \mathrm{C}$ at 4 weeks, implying that high temperature in curing deteriorated fly ash cement mortar samples is efficient for self-healing performance. The fly ash has a pozzolanic reaction that reacts with calcium hydroxide from cement hydration and produces C-S-H gel, which may heal micro cracks under high temperature curing condition. Consequently, it is expected that the mortar made with cement and fly ash may improve various properties such as compressive strength, bending strength and RDM, showing self-healing ability. These results agree with the findings in the previous work (Pipat et al. 2009).

Figure 11 shows the relationship between accelerated carbonation time and carbonation depth for specimens FA30, which include $30 \%$ by volume fly ash replacement ratio, considering the four studied cases. It is clear from the figure that the carbonation depth of sample FA30 in the case of curing in water $20^{\circ} \mathrm{C}$ at 1 week shows almost the same response as the case after deterioration. However, the sample carbonation depth, in the case that it is cured in water $40^{\circ} \mathrm{C}$ at 4 weeks, is less than $1 \mathrm{~mm}$. Also, it is found that the carbonation depth for the case cured in water $40^{\circ} \mathrm{C}$ at 4 weeks is smaller than no cracking case, at any time of accelerated carbonation test, implying that the curing condition of mortar with fly ash would affect the carbonation depth and self-healing. The fact that curing in water at high temperature would result in continuing the hydration of both unhydrated cement and fly ash, which can fill micro cracks is the cause of the observed performance in FA30 mortar sample for carbonation test.

The carbonation coefficient for each studied case of the designated mixtures is given in Fig. 12. It can be inferred from the figure that mixtures incorporating fly ash show smaller carbonation coefficient and better performance compared to the mixtures without fly ash 
under curing condition of $40^{\circ} \mathrm{C}$ at 4 weeks. The reason that incorporating fly ash in mortar would result in higher resistance to carbonation effect is because of the pozzolanic reaction of fly ash. The pozzolanic reaction of fly ash may continue after the full hydration of cement, and consequently, micro cracks can be filled by hydrated products of fly ash resulting in higher resistance to carbonation test.

Figure 13 shows the effect of water to cement ratio on the carbonation coefficient in fly ash mortar samples FAX, FA20 and FAY, which are with same condition of type and content of fly ash but water to cement ratios, for different curing conditions. It is found that the fly ash mortar samples with lower water to cement ratio lead to smaller carbonation coefficient and better self-healing performance for the studied cases. The fact that smaller water to cement ratio may cause smaller volume of micro cracks and pores in mortar sample, which is enough to be sealed by hydration products of cement and fly ash, is the reason for this better self-healing ability and carbonation resistance. On the other hand, larger water to cement ratio may contribute into a large volume of micro cracks and pores in mortar sample, which requires more hydration products of cement and fly ash to be filled in order to have more resistance for carbonation test.

The values of carbonation coefficient for two mortar mixes, which are N (OPC mortar mix), FA20 (fly ash cement mortar mix with $20 \%$ by volume replacement ratio), are given in Fig. 14. Also, the figure shows the results of carbonation coefficient for seven cases of each examined mortar mixes, which are no cracking case, after deterioration (RDM 60\%) case, cured in water $20^{\circ} \mathrm{C}$ at 1 week (RDM $60 \%$ ) case and cured in water $40^{\circ} \mathrm{C}$ at 4 weeks (RDM $60 \%$ ) case, after deterioration (RDM $80 \%$ ) case, cured in water $20^{\circ} \mathrm{C}$ at 1 week (RDM $80 \%$ ) case and cured in water $40^{\circ} \mathrm{C}$ at 4 weeks (RDM $80 \%$ ) case. It can be seen from the figure that fly ash mortar sample FA20 shows smaller carbonation coefficient under curing condition $40^{\circ} \mathrm{C}$ in water at 4 weeks for both deterioration indexes $60 \%$ and $80 \%$ compared to OPC mortar mix $\mathrm{N}$ for the same curing condition. This behavior can be attributed to the fact that fly ash is a pozzolanic active material that reacts with calcium hydroxide in cement producing C-S-H gel efficiently under high temperature curing condition in water, which may fill micro cracks in the fly ash mortar mixtures, and, thus, improve the self-healing performance and resistance to carbonation.

\subsection{Pore structure investigation and point count}

Figures 15 (a), (b) and (c) show the change in relative pore volume with respect to pore size for three mortar samples N, HL and FA20, respectively. These results which were obtained by mercury intrusion porosimetry (MIP) technique, for four different cases which are no cracking case, after deterioration case, cured in water $20^{\circ} \mathrm{C}$ at 1 week case and cured in water $40^{\circ} \mathrm{C}$ at 4 weeks

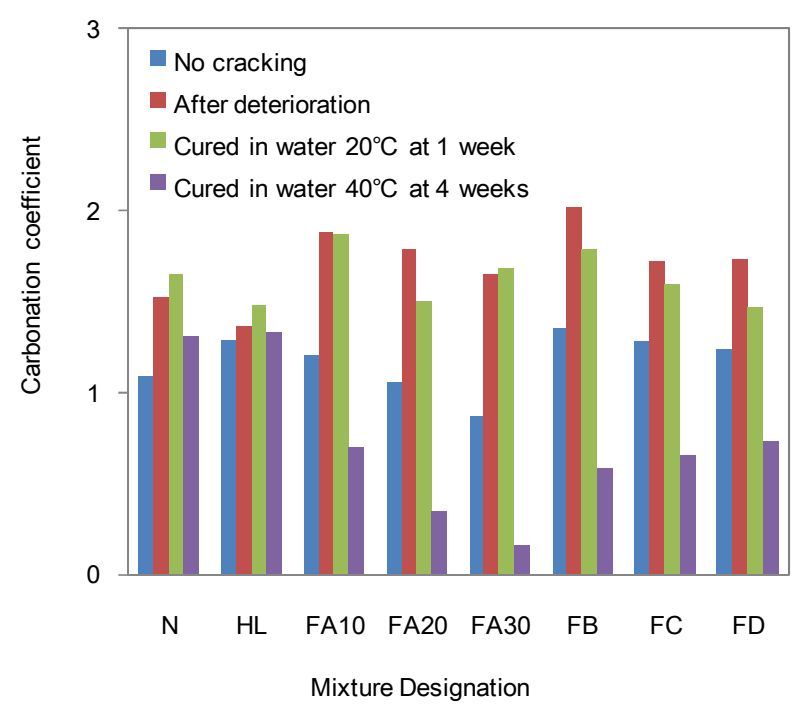

Fig. 12 Self-healing effects on carbonation coefficient for different mixtures and curing conditions.

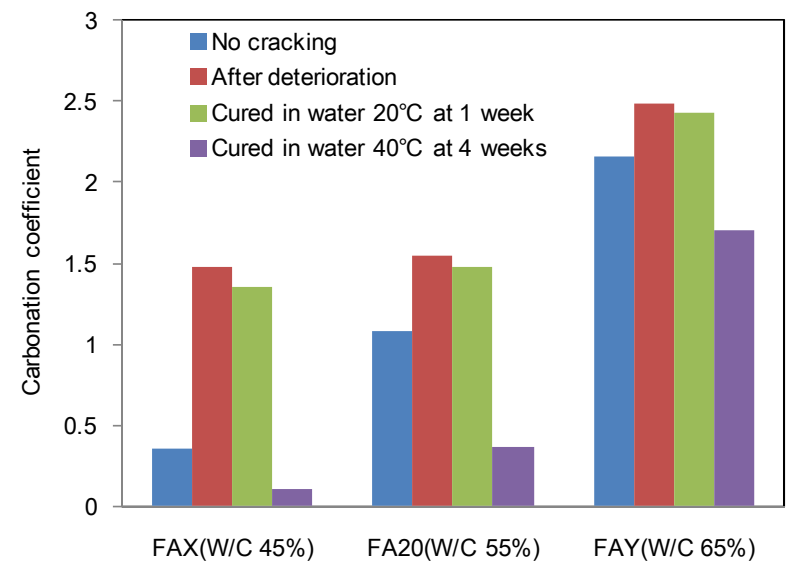

Fig. 13 Change of carbonation coefficient for fly ash mortar samples with different water to cement ratio subjected to $\mathrm{CO}_{2}$ for 13 weeks after drying.

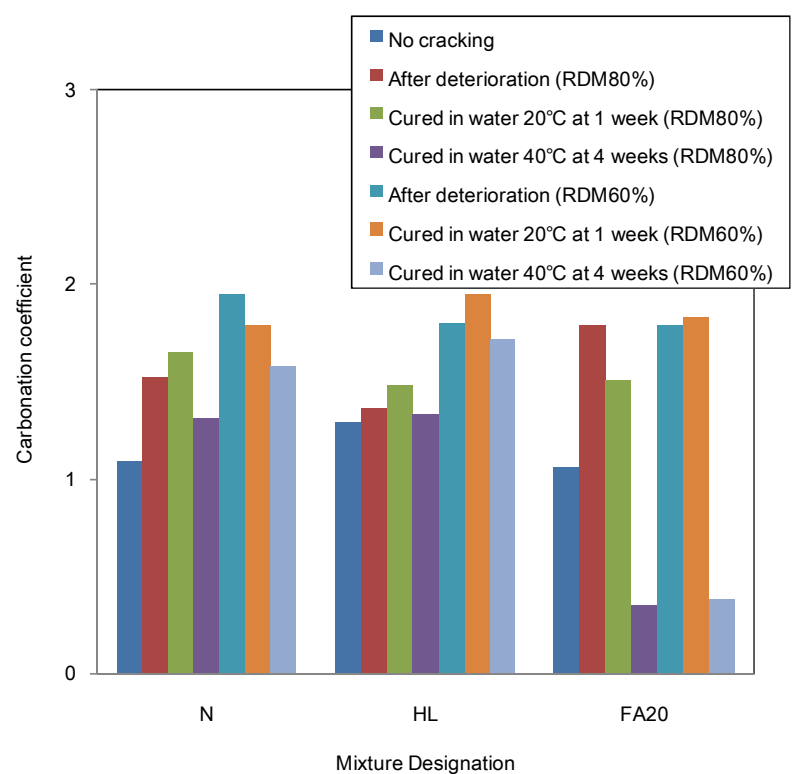

Fig. 14 Change of carbonation coefficient for different damage degrees and curing conditions. 


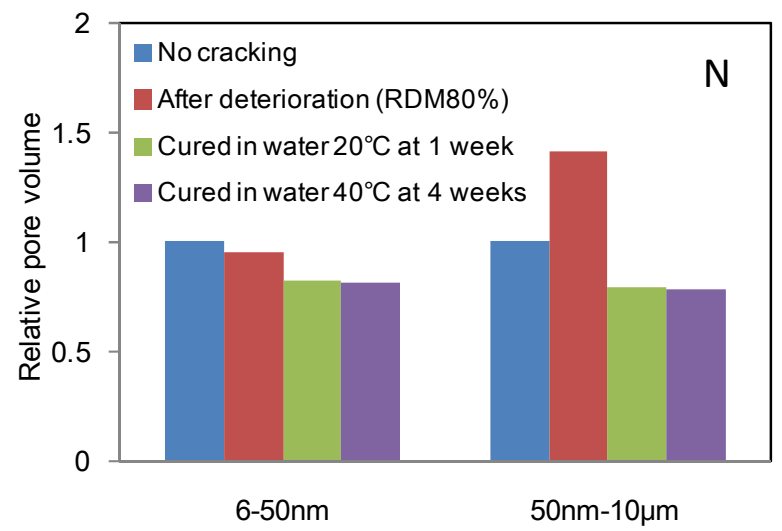

Pore diameter

(a) $\mathrm{N}$ sample

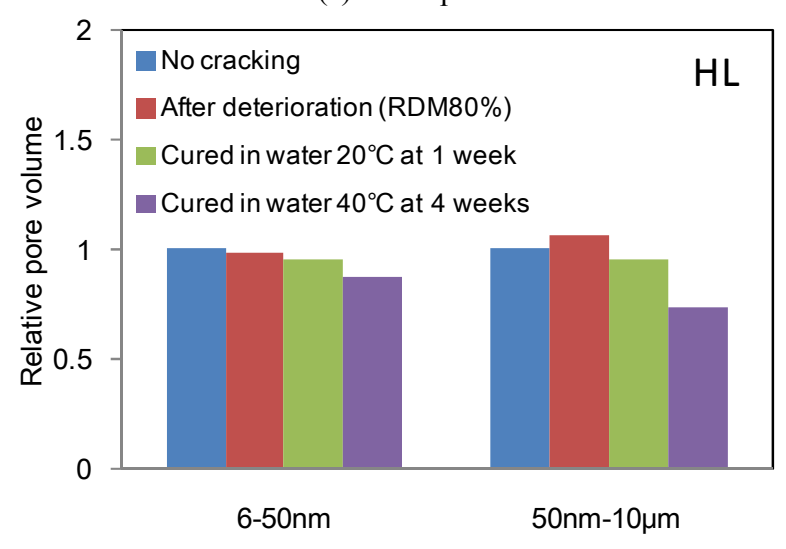

Pore diameter

(b) HL sample

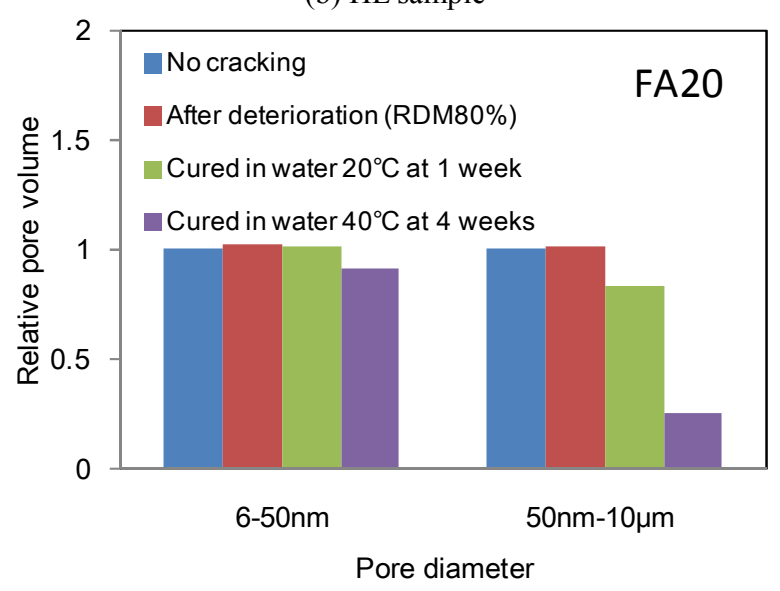

(c) FA 20 sample

Fig. 15 Change of pore volume of mortar mixtures for different curing conditions.

case are given in the figure. It should be noticed that mortar samples HL and FA20 incorporate a combination of high early strength Portland cement type $(\mathrm{H})$ and low heat Portland cement (L), while mortar sample $\mathrm{N}$ incorporates OPC cement. In addition, mortar sample FA20 incorporates fly ash with $20 \%$ replacement ratio. Pore size distributions were examined; the pore sizes were divided into two ranges, 6 to $50 \mathrm{~nm}$ of meso pores

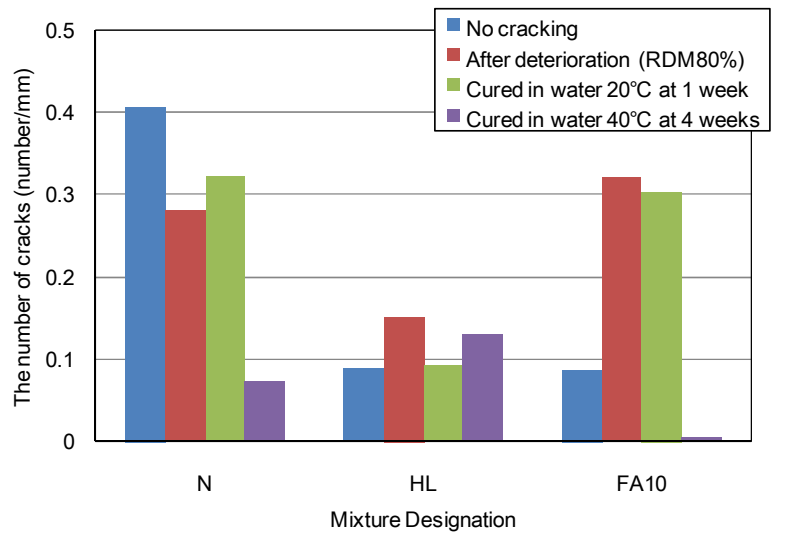

Fig. 16 Change of the number of cracks with mortar mixtures for different curing conditions.

and lager than $50 \mathrm{~nm}$ to $10 \mu \mathrm{m}$ of micro pores. (F. de Larrard 2002 and M.M.Y. Delmi 2004). It is obvious from Fig. 15 that the change in ratio of pore volume for 6 to $50 \mathrm{~nm}$ pore size is almost the same between N, HL and FA20 mortar mixes. While after deterioration, the rate of pore volume for $50 \mathrm{~nm}$ to $10 \mu \mathrm{m}$ pore size for $\mathrm{N}$ mortar sample increased in comparison to HL and FA20 mortar samples, this is due to using of a combination of $\mathrm{H}$ and $\mathrm{L}$ cement types. A notable reduction of pore volume rate for $50 \mathrm{~nm}$ to $10 \mu \mathrm{m}$ pore size in fly ash mortar sample FA20 was obtained for $40^{\circ} \mathrm{C}$ curing condition in water at 4 weeks case. The reason for this performance is that the fly ash has a pozzolanic ability that produces C-S-H gel, which may fill $50 \mathrm{~nm}$ to $10 \mu \mathrm{m}$ pore size under high temperature curing condition.

Figure 16 shows change of the number of cracks per unit length obtained by point count test for N, HL and FA10 mortar samples for different curing conditions after $80 \%$ deterioration of relative dynamic modulus of elasticity. It is clear that FA10 sample, in comparison with $\mathrm{N}$ and $\mathrm{HL}$ samples, experienced greater reduction in the number of cracks for $40^{\circ} \mathrm{C}$ curing condition in water at 4 weeks case with respect to the number of cracks for after deterioration case (RDM 80\%). This observation implies that products of fly ash can fill micro cracks under high temperature curing condition, as an evidence for self-healing phenomena and self-healing effect on micro cracks.

\subsection{Optimization of fly ash replacement ratio}

The above results clarified that the behavior of fly ash is attributed to the pozzolanic reaction due to the glass content of acid insoluble. This glass content can be obtained by deducting the amount of crystalline from the acid insoluble matter in the fly ash. The pozzolanic reaction may continue after the full hydration of cement, and consequently, remaining pores can be filled by this pozzolanic reaction. Figure 17 shows the calculated values of reaction rate of fly ash and cement minerals in fly ash blended cement paste (as discussed in section 3.3.2) for which the temperature and relative humidity 


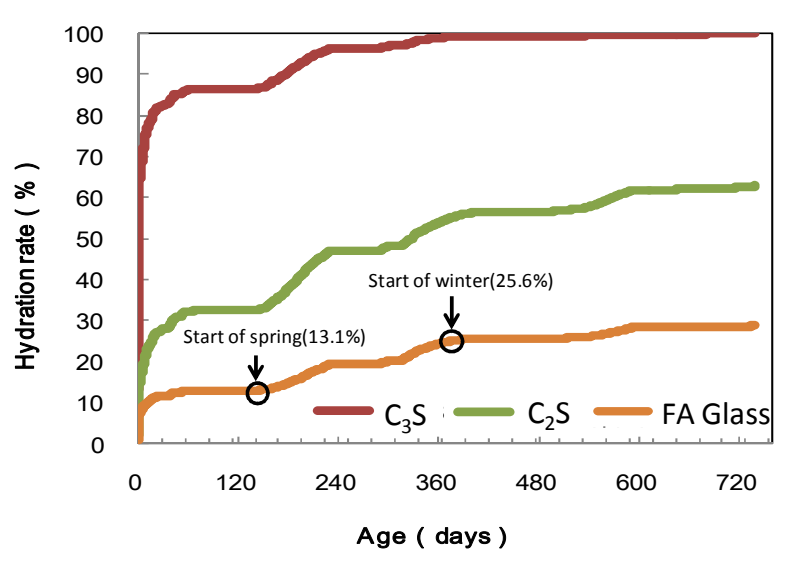

Fig. 17 Calculating reaction rate of fly ash and cement minerals based on measurement of environment temperature and humidity until 2 years.

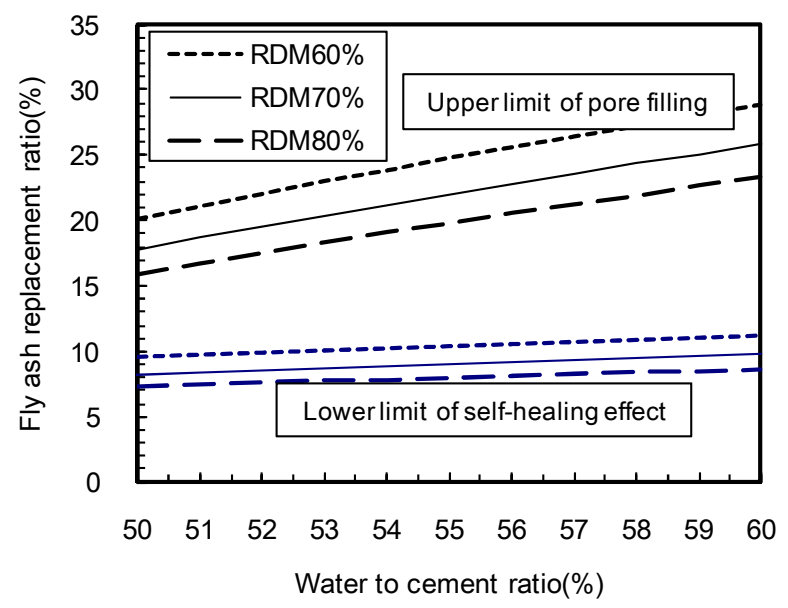

Fig. 18 Relationship between fly ash replacement ratio and water cement ratio for different damage degrees.

have been measured for 2 years. It is obvious that the hydration rate of fly ash and $\mathrm{C}_{2} \mathrm{~S}$ continues, even, after full hydration of $\mathrm{C}_{3} \mathrm{~S}$ at the time of 360 days. It is known that when the relative dynamic modulus of elasticity was about $60 \%$, the relative length change was about $1 \times 10^{-3}$ due to fact that internal cracking in the concrete sample took place for the rapid freezing and throwing test (Koh and Hasegawa 1981). Therefore, to obtain optimization of fly ash replacement ratio in concrete, it is assumed that the relative dynamic modulus of elasticity for concrete structure deteriorates until $60 \%$ and micro cracks in concrete are about $0.3 \%$ (by volume). An increase of volume by $0.3 \%$ is typical to 3 liters, and the amount of fly ash can be calculated as shown in Eqs. (12), (13) and (14).

$$
V=V_{F A}\left(R_{\text {winter }}-R_{\text {spring }}\right) / 100 \times G
$$

Where;

$\mathrm{V}=$ increase of volume by 3 liters due to micro cracks

$\mathrm{V}_{F A}=$ increase of volume by fly ash reaction, equals to 2.4 which was determined based on the current experi- ment)

$R_{\text {winter }}=$ percentage of fly ash reaction in start of winter (see Fig. 17), and it is equal to $25.6 \%$

$R_{\text {spring }}=$ percentage of fly ash reaction in start of spring (see Fig. 17), and it is equal to $13.1 \%$

$\mathrm{G}=$ glass phase of fly ash and it can be expressed as [Fw(Fins-Fcry)/(100 $\rho f)]$

Hence, Eq. (12) can be rewritten as follows,

$$
3=2.4(25.6-13.1) / 100 \times F w(\text { Fins }- \text { Fcry }) /(100 \rho f)
$$

$$
F w=3 /(2.4 \cdot 0.124(\text { Fins }-F c r y) /(100 \rho f))
$$

Where, $F w$ is the fly ash content $\left(\mathrm{kg} / \mathrm{m}^{3}\right)$, Fins is insoluble residue of fly ash (\%), Fcry is the crystal content in fly ash (\%) and $\rho f$ is density of fly ash $\left(\mathrm{g} / \mathrm{cm}^{3}\right)$, the fly ash substitution ratio $F / B$ can be calculated from Eq. (15).

$$
F / B(\%)=F w /(C w+F w) \times 100
$$

Where, $F w$ is fly ash content $\left(\mathrm{kg} / \mathrm{m}^{3}\right)$ and $C w$ is cement unit weight $\left(\mathrm{kg} / \mathrm{m}^{3}\right)$. The fly ash reaction volume, Frv can be calculated as shown in Eq. (16).

$$
\text { Frv }=F w(\text { Fins }- \text { Fcry }) /(100 \rho f)
$$

In addition, to avoid filling pores in concrete, the maximum fly ash contents can be determined based on a concrete unit that can be represented as water unit, cement unit, density of cement and the fly ash reaction as follows:

$$
\begin{aligned}
& W w-(1.06 \cdot C w / \rho c+2.4 \cdot F r v)>0 \\
& F w<(W w-1.06 \cdot C w / \rho c) / 2.4
\end{aligned}
$$

Where, $W w$ is unit water weight $\left(\mathrm{kg} / \mathrm{m}^{3}\right), \rho c$ is density of cement $\left(\mathrm{g} / \mathrm{cm}^{3}\right)$, and $F r v$ is fly ash reaction volume.

From the above equations, the fly ash replacement ratio determines the glass content and water cement ratio which are needed to both heal the deteriorated concrete until RDM $60 \%$ and to fill pore for long periods. Figure 17 shows the hydration rate with $\mathrm{C}_{3} \mathrm{~S}$ and $\mathrm{C}_{2} \mathrm{~S}$ fly ash based on the natural temperature and humidity in internal slab specimens in Asahikawa, Japan. This hydration rate for the mineral composite was calculated by using reaction rate equation (chapter 3.3.2). From the figure, the hydration rate for $\mathrm{C}_{3} \mathrm{~S}$ increased until 360 days and was also constant after 360 days, however, the hydration rate of $\mathrm{C}_{2} \mathrm{~S}$ and fly ash did not completely hydrated at 720 days. Figure 18 represents the relationship between the fly ash replacement ratio and water to cement ratio in deteriorated concrete for relative dynamic modulus of elasticity (RDM) 60\%, 70\% and 80\%, which were derived from the above equations. This relationship has upper and lower limits for calculation of fly ash replacement ratio at different water to cement ratio as 
shown in the figure to ensure proper self-healing performance. Accordingly, it can be suggested that the practical fly ash replacement ratios in fly ash concrete mix could be $10 \%$ to $15 \%$ and $11 \%$ to $20 \%$ for water to cement ratios (W/C) 0.50 to 0.55 and 0.55 to 0.60 , respectively.

\section{Conclusion}

The purpose of this study is to investigate the hydration process, reaction rate of cement and fly ash in fly ash blended cement paste, and self-healing ability in mortar incorporating fly ash for long-term performance. First, the experiment examined the reaction rate of fly ash and consumption of calcium hydroxide by adding calcium hydroxide reagents to fly ash. Then, reaction rate of fly ash and remaining contents of $\mathrm{Ca}(\mathrm{OH})_{2}$ were measured based on acceleration reaction test and rate of reaction test in fly ash cement paste taking into consideration different types of fly ash, fly ash replacement ratios, curing conditions. The performance of self-healing ability of mortar samples incorporating fly ash was evaluated using ten mixtures with different types of cement, fly ash, fly ash replacement ratio, water to cement ratio and curing conditions. Mortar samples were deteriorated by freeze/thaw cycling until $60 \%$ and $80 \%$ relative dynamic modulus of elasticity to examine their characteristics properties such as compressive strength, bending strength, accelerated carbonation depth, and pore structure modification. Based on experiment results, the following conclusions can be derived, which are valuable information to enhance the existing design codes with long-term performance of self-healing ability of fly ash that is quite needed to prolong the service life of concrete structures:

(1) From the hydration performance of calcium hydroxide reagent and fly ash, the calculation of reaction rate of fly ash using pattern 4 was found to have better response to experimental results compared to other patterns. The reason for this response is due to the assumption of pattern 4 , in which the reaction rate is calculated based on the melting ratio for the acid insoluble matter in the glass phase, meaning that there is no contribution of crystalline phase to reaction rate.

(2) Incorporating fly ash in cement paste would affect the hydration reaction rate and consumption of calcium hydroxide. In comparison with $\mathrm{N}$ sample, which is the case of OPC sample without fly ash replacement, the remaining contents of calcium hydroxide in fly ash cement samples after 2 days decreased because of its pozzolanic reaction. For the remaining calcium hydroxide contents at each age, it was found that there is no significant difference among the types of fly ash.

(3) Fly ash cement samples with fly ash replacement ratio by mass $30 \%$, and with high curing temperature, showed smaller remaining amount of calcium hy- droxide compared to other samples with smaller fly ash replacement ratio. This implies that large amount of fly ash as 30\% replacement ratio and high curing temperature in water can increase the consumption amount of calcium hydroxide. The reason for this behavior is due to the pozzolanic reaction of fly ash, which increases with increasing the amount of fly ash and curing temperature, and thus consumes more calcium hydroxide that comes from cement.

(4) It was found that reaction rate of fly ash depends on curing temperature, curing age and fly ash replacement ratio.

(5) The investigated mortar samples can heal the bending strength, compressive strength, crack density and relative dynamic modulus of elasticity after deterioration. However, degree of recovering in mortar samples incorporating fly ash was higher compared to OPC mortar sample without fly ash, especially for the case of curing in water $40^{\circ} \mathrm{C}$ at 4 weeks. Also, it was clarified that the curing condition in water $20^{\circ} \mathrm{C}$ at 1 week was not enough to heal the micro cracks compared to the curing condition in water $40^{\circ} \mathrm{C}$ at 4 weeks. The fly ash has a pozzolanic reaction that reacts with calcium hydroxide from cement hydration and produces C-S-H gel, which may heal micro cracks under high temperature curing condition.

(6) It was confirmed that the carbonation depth for the case cured in water $40^{\circ} \mathrm{C}$ at 4 weeks is less than 1 $\mathrm{mm}$ and smaller than the no cracking case at any time of accelerated carbonation test for fly ash mortar samples, concluding that the curing condition of mortar with fly ash would affect the carbonation depth and self-healing due to continuing the hydration of both unhydrated cement and fly ash. Incorporating fly ash in mortar would result in higher resistance to carbonation effect is because of the pozzolanic reaction of fly ash. The pozzolanic reaction of fly ash may continue after the full hydration of cement, and consequently, micro cracks can be filled by hydrated products of fly ash resulting in higher resistance to carbonation test.

(7) The fly ash mortar samples with lower water to cement ratio resulted in smaller carbonation coefficient and better self-healing performance for the studied cases. The fact that smaller water to cement ratio may cause smaller volume of micro cracks and pores in mortar sample, which is enough to be sealed by hydration products of cement and fly ash, is the reason for this better self-healing ability and carbonation resistance. On the other hand, larger water to cement ratio may contribute into a large volume of micro cracks and pores in mortar sample, which requires more hydration products of cement and fly ash to be filled in order to have more resistance for carbonation test.

(8) The reductions of pore volume rate $50 \mathrm{~nm}$ to $10 \mu \mathrm{m}$ 
in fly ash mortar sample FA20 were higher than those in ordinary Portland cement sample $\mathrm{N}$, for $40^{\circ} \mathrm{C}$ curing condition in water at 4 weeks case. The reason for this behaviour is that the fly ash has a pozzolanic ability that produces $\mathrm{C}-\mathrm{S}-\mathrm{H}$ gel, which may fill $50 \mathrm{~nm}$ to $10 \mu \mathrm{m}$ pore size under high temperature curing condition. All the above results show the potential performance of fly ash as a pozzolanic material that can improve the self-healing ability.

(9) Based on the practical assumption and to ensure proper self-healing performance, practical fly ash replacement ratios in fly ash concrete could be $10 \%$ to $15 \%$ and $11 \%$ to $20 \%$ for water to cement ratios 0.50 to 0.55 and 0.55 to 0.60 , respectively.

\section{Acknowledgement}

The authors would like to convey their deepest gratitude to the priority research project, Hokkaido Prefecture, Japan, for the support and fund used in this study.

\section{References}

Asaga, K., Ishizaki., M., Takahashi, K. and Konishi, K., (1992). "Effect of curing temperature on the hydration of portland cement compounds." In: 9th International Congress on the Chemistry of Cement, 4, 181-187.

de Larrard, F., (2002). Construire en beton, ENPC Press, Paris.

Delmi, M. M. Y., (2004). Etude de l'hydratation et du couplage carbonatation-echanges hydriques dans les mortiers et betons. PhD Thesis of the University of $L a$ Rochelle.

Hoshino, S., Hirao, H., and Yamada, K., (2005). "The application of XRD/Rietveld method to cements with amorphous admixture as quantitative tool of mineral composition." Cement Science and Concrete Technology, 59, 14-21. (in Japanese)

Itoh, T., Masaki, K. and Igarashi, H., (2002). "Relationship between property of early hydration of cement and aluminate contents determined by rietveld method." Cement Science and Concrete Technology, 56, 29-35. (in Japanese)

Izumi, F. and Ikeda, T., (2000). "A Rietveld-analysis program RIETAN-98 and its application to Zeolites." Mater. Sci. Forum, 321-324.

JCI, (2009). "Technical committee reports on autogenous healing in cementitious materials." Tokyo, Japan Concrete Institute. (in Japanese)

Koh, E. and Hasegawa, T., (1981), "On the test method for resistance of concrete to freezing and thawing." Concrete Journal, 16 (9), 16-22. (in Japanese)

Matsui, J. and Ikabata, T., (1999). "Hydration rate and strength development of low heat portland cement with large amount of pozzolan." Cement Science and Concrete Technology, 53, 229-236. (in Japanese)
Matsumura, T. Katsura O. and Yoshino, T., (2003), "Properties of frost damaged concrete and the estimation of the degree of frost damage." Journal of Structural and Construction Engineering, Transaction of AIJ, 563, 9-13.

Ohsawa, S., Sakai, E. and Daimon, M., (1999). "Reaction ratio of fly ash in the hydration of fly ash-cement system." Cement Science and Concrete Technology, 53, 96-101. (in Japanese)

Saeki, T., and Monteiro, P. J. M., (2004). “A model to predict the amount of calcium hydroxide in concrete containing mineral admixtures." Cement and Concrete Research, 35(10), 1914-1921.

Saeki, T. and Nagataki, S., (1997). "Fundamental study on reaction between fly ash and calcium hydroxide." JCA proceedings of cement and concrete, 51, 162-167. (in Japanese)

Sagawa, T., Katsura, O., Sekiguchi, T. and Wakasugi, S., (2004). "Effect of water to cement ratio and curing condition on hydration of portland cement compounds." Cement Science and Concrete Technology, 58, 23-30. (in Japanese)

Sagawa, T. Katsura, O., Sekiguchi, T. and Wakasugi, S., (2005). "Hydration rate of portland cement compounds and strength development in consideration of particel size distribution." Cement Science and Concrete Technology, 59, 45-52. (in Japanese)

Scrivener, K.L., Füllmann, T., Gallucci, E., Walenta, G. and Bermejo, E., (2004). "Quantitative study of portland cement hydration by X-ray diffraction/ Rietveld analysis and independent methods" Cement and Concrete Research, 34(9), 1541-1547.

Termkhajornkit, P., Nawa, T., Nakai, M. and Saito, T., (2005). "Effect of fly ash on autogenous shrinkage." Cement and Concrete Research, 35(3), 473-482.

Termkhajornkit, P., Nawa, T., Yamashiro, Y. and Saito, T., (2009). "Self-healing ability of fly ash-cement systems." Cement and Concrete Composites, 31, 195-203.

Whitfield, P. S. and Mitchell, L. D., (2003). "Quantitative Rietveld analysis of the amorphous content in cements and clinkers." Journal of Materials Science, 38(21), 4415-4421.

Yamaguchi, G., Takemoto, H. and Uchikawa, H., (1960). "Rate of hydration of cement compounds and portland cement estimated by X-ray diffraction analysis." In: 4th International Symposium on Chemistry of Cement, 1, 496-499.

Yamamoto, T. and Kanazu, T., (2004). "Investigation on the pozzolanic reaction of fly ash-clarification of the mechanism of pozzolanic reaction and optimization of accelerated chemical test (API method)." Laboratory Rep. No.N04008, Central Res. Inst. of Electric Power Industry, Civil Engineering Research. 


\title{
Self Healing of Longitudinal Cracks in Utility Concrete Pole
}

\author{
Akira Hosoda $^{1}$, Takayuki Higuchi ${ }^{2}$, Masataka Eguchi ${ }^{3}$, Haruaki Yoshida ${ }^{4}$ and Hayato Aoki ${ }^{5}$
}

\begin{abstract}
The precipitates in cracks in an actual utility concrete pole exhibiting self healing which had been in service for 34 years in a freezing and thawing environment were analyzed and the mechanism of self healing was discussed. $\mathrm{CaCO}_{3}$ was observed at the surface of the pole, and it was guessed from the condition of precipitation that water accumulated inside the pole had flowed through the crack to the surface. $\mathrm{CaCO}_{3}$ and ettringite were precipitated in the cracks inside the pole. While cracks approximately $200 \mu \mathrm{m}$ wide were not completely healed, cracks with a smaller width of 50 to $100 \mu \mathrm{m}$ were blocked up with precipitates. Precipitates were mainly observed near the interface between the matrix and crack, and much less near the interface between the aggregate and crack. The steel inside the concrete was protected against corrosion by self healing of cracks.
\end{abstract}

\section{Introduction}

Utility concrete poles are indispensable members in the infrastructure network for power supply. Recently, deterioration conditions of utility prestressed concrete poles have been precisely investigated, and longitudinal cracks, net shape cracks, spalling of concrete, and corrosion of steel due to freezing and thawing or/and chloride ingress, have been clarified (Yoshida 2007). An investigation to clarify the mechanism of generation and development of longitudinal cracks in PC utility poles has also been conducted (Watanabe et al. 2011).

On the other hand, self healing of cracks in PC poles which controlled the progress of deterioration has been reported (Yoshida 2007). This natural self healing is included in the self healing phenomena of concrete (JCI 2009,2011 ) and may contribute to the long life of poles and the reduction of maintenance cost. Self healing of cracks in a marine environment has been reported and it was reported that the corrosion of steel bars in concrete was prevented due to the formation of ettringite, Friedel's salt, and magnesium hydroxide in cracks (Mohammed and Hamada 2008). However, no research has been conducted on the detailed mechanism of self healing of prestressed concrete poles.

${ }^{1}$ Institute of Urban Innovation, Yokohama National University, Yokohama, Japan.

E-mail: concrete@ynu.ac.jp

${ }^{2}$ Inorganic Material Research Laboratory, Omi Plant, Denki Kagaku Kogyo Kabushiki Kaisha, Itoigawa, Niigata Pref., Japan.

${ }^{3}$ Inorganic Material Research Laboratory, Omi Plant, Denki Kagaku Kogyo Kabushiki Kaisha, Itoigawa, Niigata Pref., Japan.

${ }^{4}$ Nihon Network Support Co., Ltd., Osaka, Japan.

${ }^{5}$ Graduate School of Environment and Information Sciences, Yokohama National University, Yokohama, Japan.
To yield a detailed understanding of the self healing of cracks in PC poles and to contribute to the improvement and utilization of the self healing function, this study investigated an actual concrete pole with self healing of cracks, analyzing the components, shape, and distribution of precipitates to shed light on the healing mechanism.

\section{Outline of analysis of concrete pole}

\subsection{Target for analysis}

Figures 1 and $\mathbf{2}$ show the target concrete pole selected for analysis in this research. The water to cement ratio of the concrete was $35 \%$, and the design compressive strength of the concrete was $60 \mathrm{~N} / \mathrm{mm}^{2}$. The pole was fabricated by centrifugal force casting and steam curing. The concrete did not contain AE agent. Rebars of tensioned PC steel, not tensioned steel, and spiral steel hoops were arranged inside the pole, and prestress was introduced by pre-tension to control cracking. The pole diameter was $190 \mathrm{~mm}$ at the top and $357 \mathrm{~mm}$ at the ground surface. The pole had been in service for 34 years in a freezing and thawing environment in Kano, Aisho
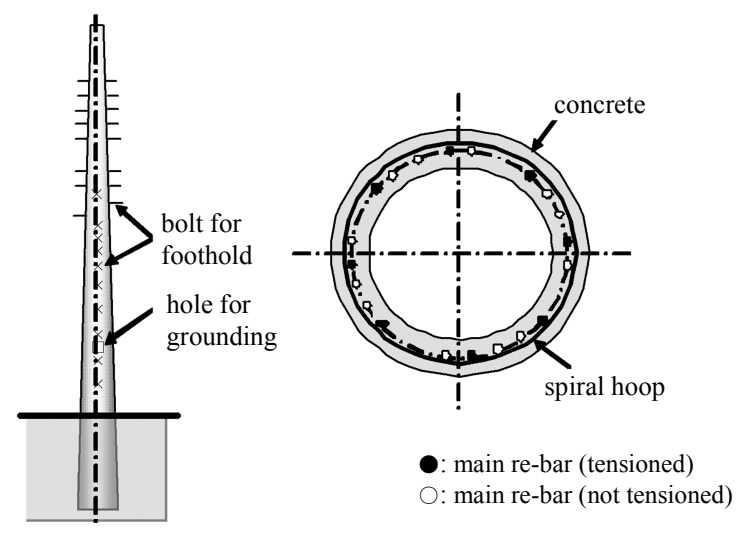

Fig. 1 Shape and section of the utility concrete pole (section at the ground). 


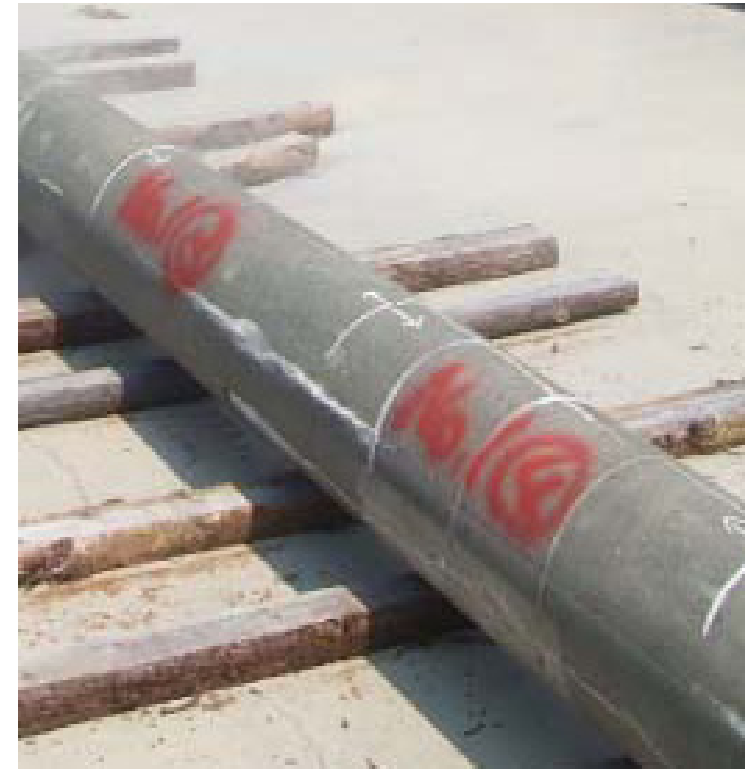

Fig. 2 Picture of the pole.

Town, Echi County, Shiga Prefecture, Japan. The pole had longitudinal cracks in the area of the joints of 2 semicircular steel forms used for fabrication, and self healing of the longitudinal cracks was observed. Smaller longitudinal cracks than those in the area of the joints were also observed, many of which had healed with white precipitation. Cracks were generated only in the longitudinal direction due to the effect of prestress. A core of $100 \mathrm{~mm}$ diameter and $80 \mathrm{~mm}$ thickness that included self healing cracks was taken from the pole. The core was taken at the height of $1.7 \mathrm{~m}$ from the ground and $3.7 \mathrm{~m}$ from the bottom of the pole. The core was taken at that location because typical and sufficient precipitation along longitudinal cracks in the area of the joint were observed there, making this location a good candidate for investigation of the effect of self healing to prevent the corrosion of the steel inside.

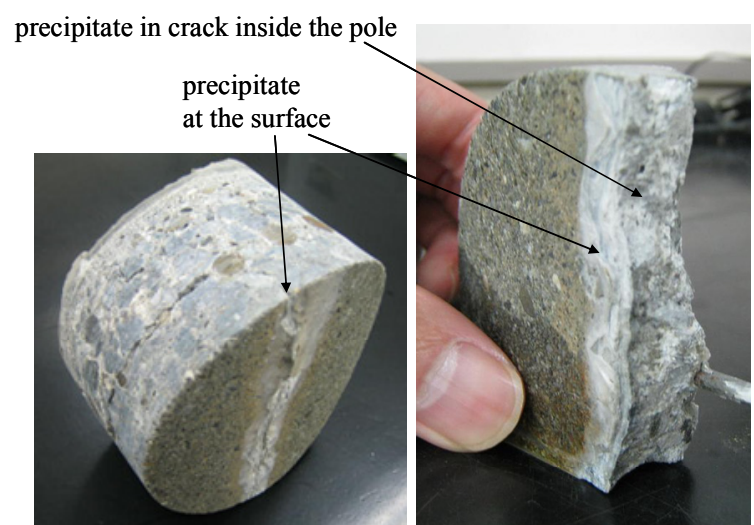

Fig. 3 Core specimen taken from the pole.

\subsection{Analysis method and pre-treatment of specimen}

(1) Powder X ray diffraction

The core taken from the pole is shown in Fig. 3. White precipitates were picked up at two locations. One was picked up at the surface of the pole, and the other from the crack plane inside the pole as shown in the right side photo of Fig. 3. White precipitates were scratched off using a spatula and crushed in an agate pot, and then analyzed by powder $\mathrm{X}$ ray diffraction.

(2) Scanning electron microscope observation Precipitates taken at two locations were observed by SEM.

(3) EPMA analysis

Element mapping by EPMA was conducted to investigate the distribution of precipitates inside the cracks in the utility pole. As shown in Fig. 4, a core from the pole was sliced with $10 \mathrm{~mm}$ thickness in the plane perpendicular to the PC steel, and the sliced specimen was embedded in epoxy resin in a vacuum ventilator. Epoxy
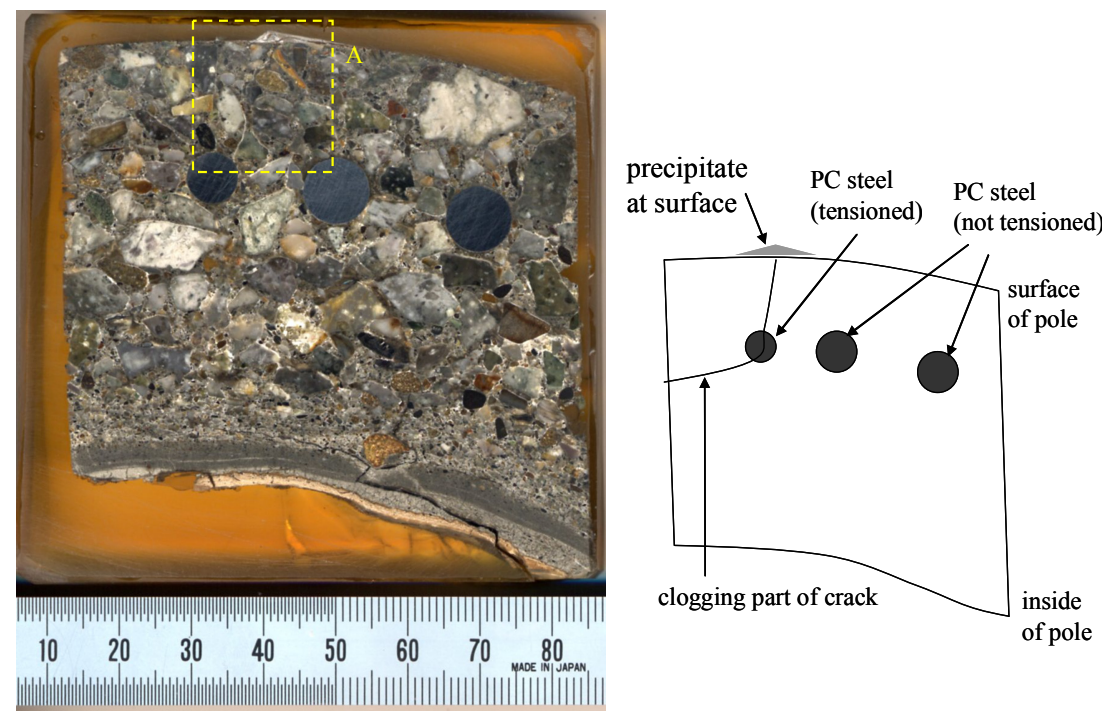

Fig. 4 Specimen for EPMA analysis and field for observation. 


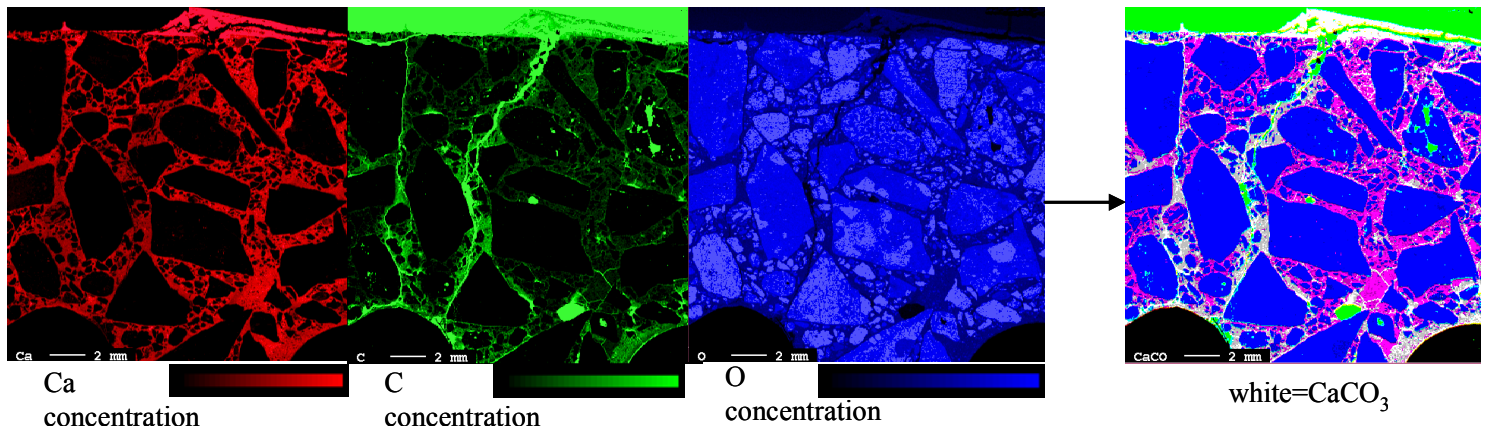

Fig. 5 Confirmation of $\mathrm{CaCO}_{3}$ precipitation by $\mathrm{RGB}$ method.

resin was poured into the cracks and voids so that the shape of the specimen with cracks became stable and the surface for EPMA observation became smooth. Voids with epoxy resin can be easily detected as voids in EPMA. The target plane was polished with water-resistant polishing paper (\#240-1200) and diamond paste $(3 \mu \mathrm{m}$ and $1 \mu \mathrm{m})$, and after that it was treated by carbon shadowing.

Area A in Fig. 4, where much precipitation was observed, was analyzed by EPMA. The diameter of the irradiation beam of EPMA was $5 \mu \mathrm{m}$, and the element concentration and reflection electron pattern of $\mathrm{Si}, \mathrm{Ca}, \mathrm{Al}$, $\mathrm{Fe}, \mathrm{C}, \mathrm{O}, \mathrm{S}$ were analyzed. Furthermore, image analysis by the RGB method was conducted in order to clarify the

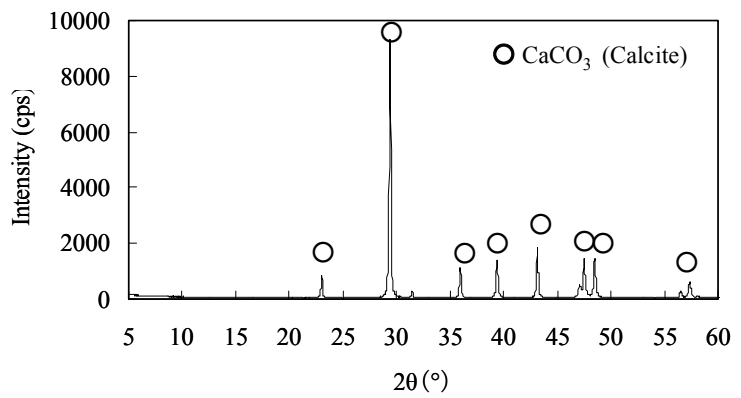

Fig. 6 Result of powder $X$ ray diffraction of precipitate at the surface.

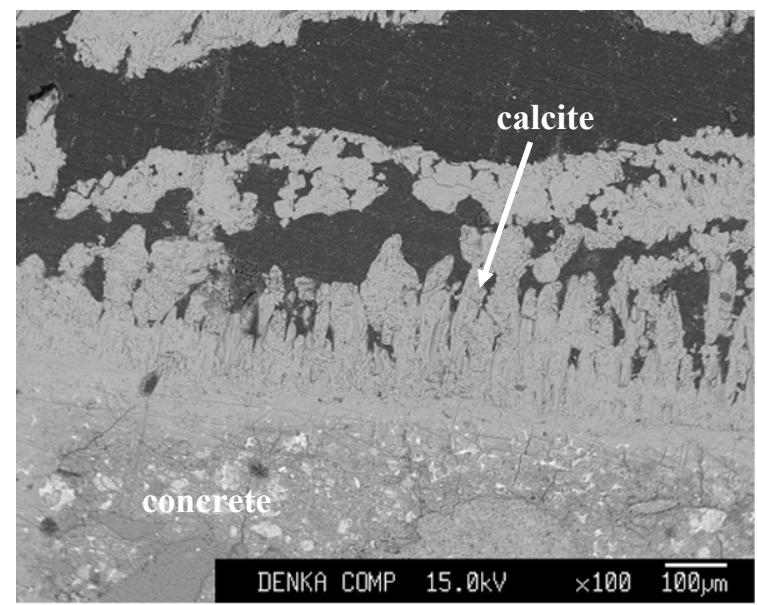

Fig. 7 Reflection electron pattern of calcite precipitated at the surface. distributions of $\mathrm{CaCO}_{3}$ and ettringite. The RGB method is a method that utilizes 3 primary colors (red, green, blue) to show specific areas that contain 3 components in a specific proportion as white colored areas. Figure $\mathbf{5}$ is an example of applying the RGB method to show $\mathrm{CaCO}_{3}$ in white color. Constituent elements such as $\mathrm{Ca}, \mathrm{C}, \mathrm{O}$ are exhibited with their concentrations in red, green, and blue, respectively, and then the images are overlapped and the areas where all 3 elements are included in a specific proportion are displayed in white color, revealing the presence of $\mathrm{CaCO}_{3}$.

\section{Results of analysis}

\subsection{Precipitate at the surface}

Figure 6 shows the result of powder X ray diffraction of the precipitate at the surface of the pole. The precipitate was calcite, one of the calcium carbonate. Figure 7 shows the reflection electron pattern of the precipitate at the surface. $\mathrm{CaCO}_{3}$ developed like the lamella of mushroom toward the outside from the outer surface of the pole. The core was taken at the height of $1.7 \mathrm{~m}$ from the ground, and the longitudinal crack was healed also in the area above and below the core portion. The water stored inside the hole of the pole flowed through the crack to the surface of the pole, when $\mathrm{Ca}$ ion was dissolved into the water, and then the water reacted with $\mathrm{CO}_{2}$ to form $\mathrm{CaCO}_{3}$. Figure 5 shows that $\mathrm{CaCO}_{3}$ can be observed almost only in cracked areas, and that almost no carbonation occurred in the healthy crack-free portion near the surface.

\subsection{Healing of crack}

As shown in Fig. 3, the core was split and the healing part was exposed, and then white precipitate was analyzed by powder $\mathrm{X}$ ray diffraction. The result is shown in Fig. 8. Besides calcite, which was observed also in the surface precipitate, vaterite (another kind of $\mathrm{CaCO}_{3}$ ) and ettringite were detected. It has been reported that vaterite could be precipitated due to carbonation of C-S-H with low content of $\mathrm{Ca}$ (Tatematsu 1992). In this research, vaterite was not observed at the surface of the pole but only in the precipitate in the cracks. This might be because the concentration of $\mathrm{Ca}$ was low inside the pole due to leaching of $\mathrm{Ca}(\mathrm{OH})_{2}$ over the long service time. 


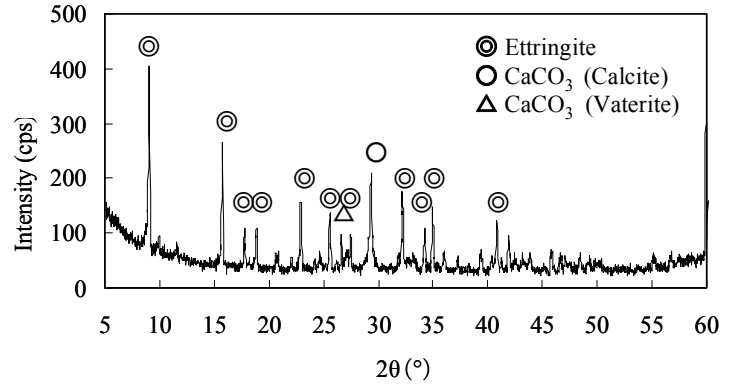

Fig. 8 Result of powder $X$ ray diffraction of precipitate in crack inside the pole.

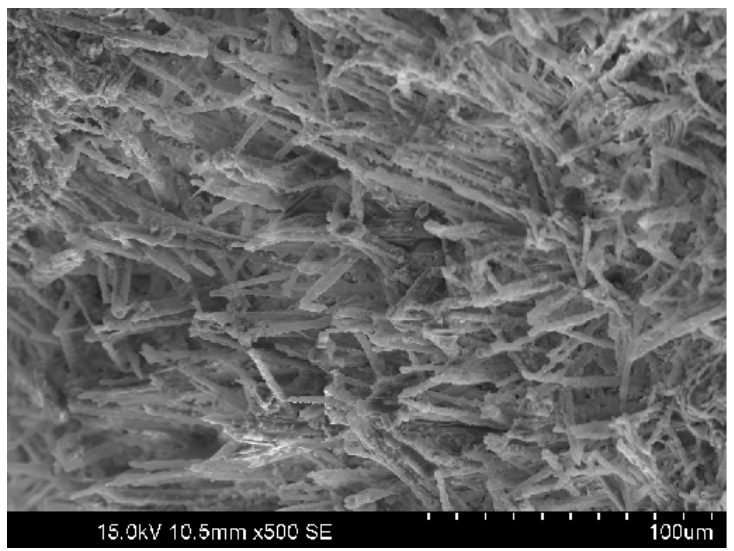

Fig. 9 SEM image of clogging part of crack.

Figure 9 shows the SEM image of the healing part. Needle shaped crystals about 50 to $100 \mu \mathrm{m}$ long along with minute particles were observed. These are ettringite and calcite, respectively. It seems that the needle shaped crystals of ettringite were precipitated in a specific direction. This specific direction might be the result of the direction of the water flowing through the crack. Looking at Fig. 7, the water in the crack flowed from inside the pole to its outer surface, judging from the direction of development of $\mathrm{CaCO}_{3}$. Constituent ions for ettringite such as $\mathrm{SO}_{4}$ were dissolved from the cement paste into the water flowing from inside the pole to its surface. Then, ettringite was gradually precipitated along the water paths in the crack, due to driving forces such as changes in concentration by drying and wetting, and changes in temperature.

Ettringite has a large amount of chemically combined water and a large volume, which makes the precipitation of ettringite effective for crack healing. The needle shaped crystals accelerated clogging inside the cracks and functioned as precipitation sites for $\mathrm{CaCO}_{3}$ because ettringite has a larger specific area.

\subsection{Element analysis inside specimen by EPMA}

Element mapping by EPMA was conducted around the PC steel and the surface of the pole in order to confirm the distributions of $\mathrm{CaCO}_{3}$ and ettringite precipitated inside the pole. The results are shown in Fig. 10. The Si concentration shows the distribution of aggregate, and the $\mathrm{Ca}$ concentration shows the distribution of the matrix.

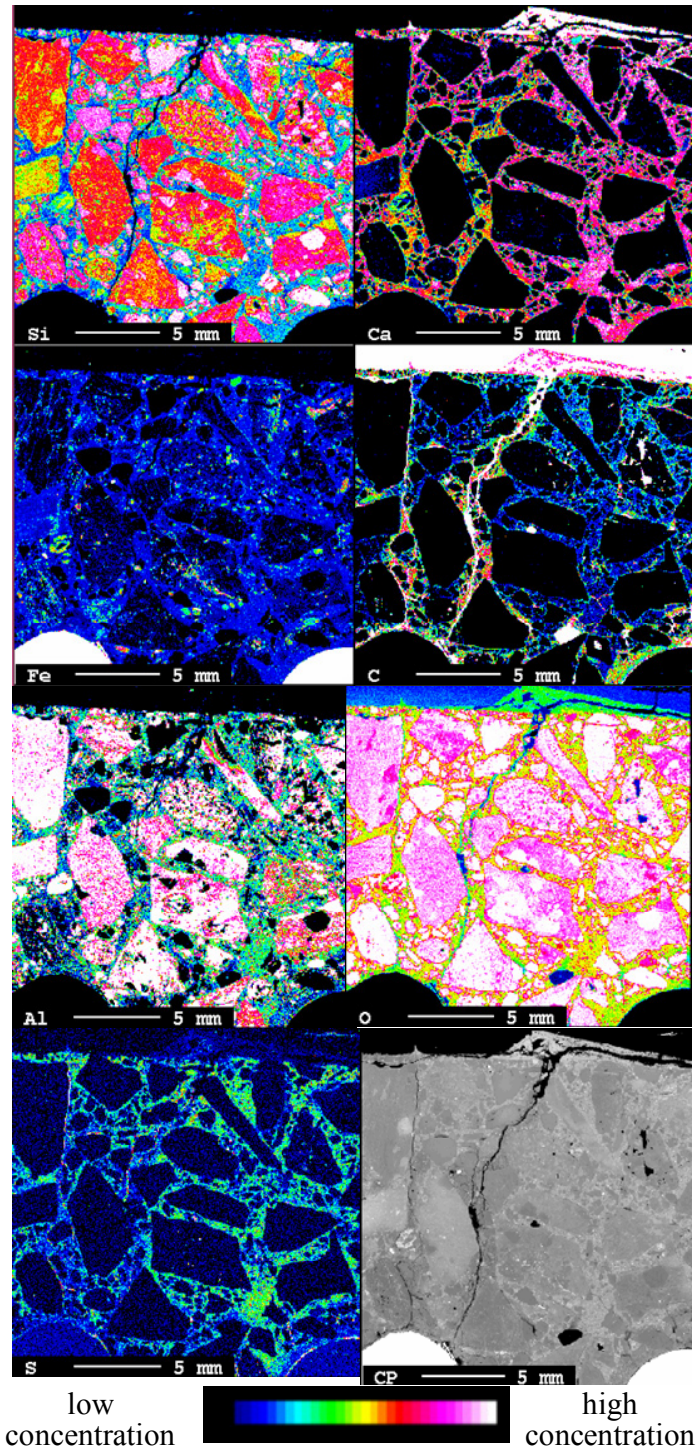

analyzed element: $\mathrm{Si}, \mathrm{Ca}, \mathrm{Fe}, \mathrm{C}, \mathrm{Al}, \mathrm{O}, \mathrm{S}$, $\mathrm{CP}$ (reflection electron pattern)

Fig. 10 Results of EPMA analysis.

The reflection electron pattern (CP) shows a black narrow road from the PC steel to the surface of the pole. The reflection electron pattern shows the areas of low density in black, and the areas of high density in white. Therefore, the black narrow road is showing low-density epoxy resin in cracks .

The distribution of $\mathrm{C}$ shows high concentration from the PC steel to the surface of the pole. This high concentration is due to epoxy resin in the cracks or $\mathrm{CaCO}_{3}$ precipitated in the cracks.

\subsection{Determination of distribution of $\mathrm{CaCO}_{3}$ by RGB method}

In order to clearly understand the distributions of $\mathrm{CaCO}_{3}$ and ettringite, image analysis by the RGB method already explained in Fig. 5 was conducted. The results are shown in Fig. 11 and Fig. 12. 
In Fig. 11, $\mathrm{CaCO}_{3}$ was exhibited in white by the RGB method. In Fig. 11(a), the blue areas show aggregate with less $\mathrm{Ca}$ or $\mathrm{C}$, the green areas show epoxy resin mainly with $\mathrm{C}$, and the pink areas show the matrix with much $\mathrm{Ca}$ but less $\mathrm{C}$. If we look at the precipitate at the surface of the pole, the white area was confirmed as $\mathrm{CaCO}_{3}$ as shown in Fig. 6. The RGB method can thus clarify the area where $\mathrm{CaCO}_{3}$ exists. In the narrow path from the precipitate at the surface to the PC steel, green, grey, and white are intermingled in some areas, which means that remaining pores, leached $\mathrm{Ca}$, and $\mathrm{CaCO}_{3}$ are intermingled.

Figure 11(b) is the result of analysis in magnified area $\mathrm{A}$ in image (a). $\mathrm{CaCO}_{3}$ with $50 \mu \mathrm{m}$ thickness was pre- cipitated along both planes of the crack. $\mathrm{CaCO}_{3}$ precipitation was more abundant at the interface between the matrix and the crack, and less abundant at the interface between the aggregate and the crack. The distribution of epoxy resin shows that the crack was not completely healed. The crack width before precipitation was around 150 to $200 \mu \mathrm{m}$. As pointed out by past research (JCI 2009, 2011), cracks with a width of about $50 \mu \mathrm{m}$ are easily healed by the precipitation of hydrates and $\mathrm{CaCO}_{3}$, but cracks with a width of about $200 \mu \mathrm{m}$ are difficult to heal.

Figure 11 (c) is the result of analysis in magnified area $\mathrm{B}$ in (a). The crack in the center with a width of about 50 to $100 \mu \mathrm{m}$ was almost completely filled with $\mathrm{CaCO}_{3}$, because the crack width was small and the crack was

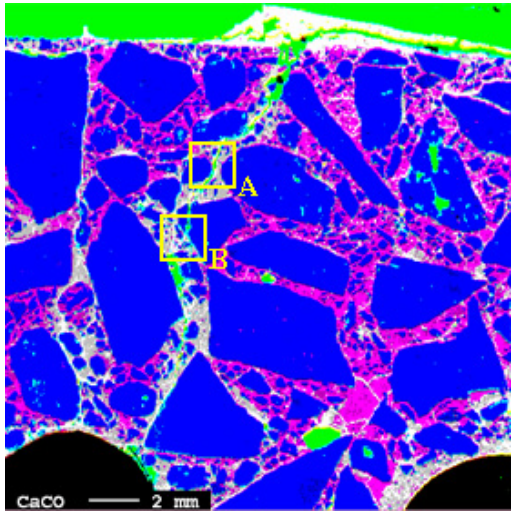

(a) near PC steel and the surface

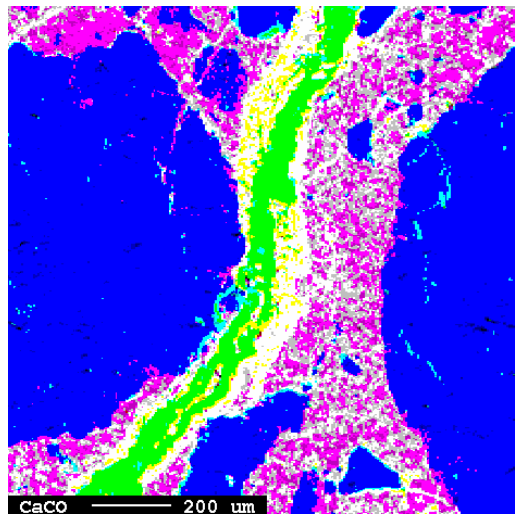

(b) magnification of area $\mathrm{A}$ in (a)

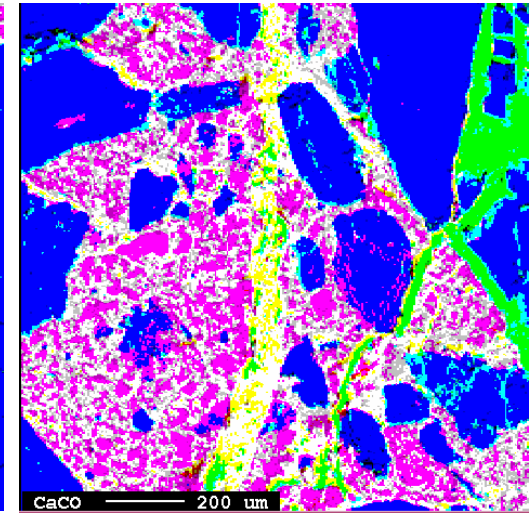

(c) magnification of area $B$ in (a)

(white: $\mathrm{CaCO}_{3}$ containing $\mathrm{Ca}, \mathrm{C}, \mathrm{O}$, pink: matrix with $\mathrm{Ca}, \mathrm{O}$, green: epoxy resin mainly with $\mathrm{C}$, blue: aggregate with $\mathrm{O}$ and with less $\mathrm{Ca}$ and $\mathrm{C}$ )

Fig. 11 Distribution of $\mathrm{CaCO}_{3}$ by $\mathrm{RGB}$ method.

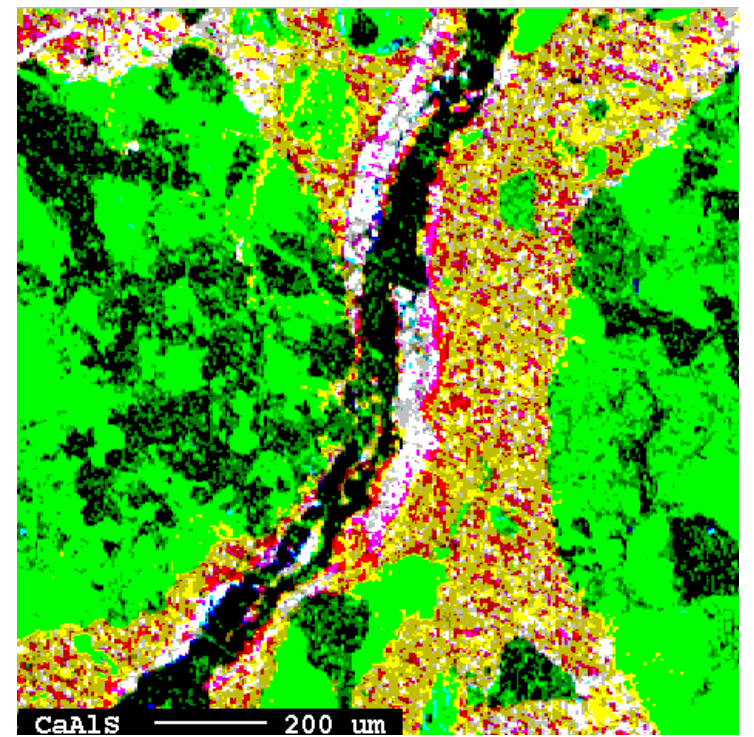

(a) area $\mathrm{A}$ in Fig. 11

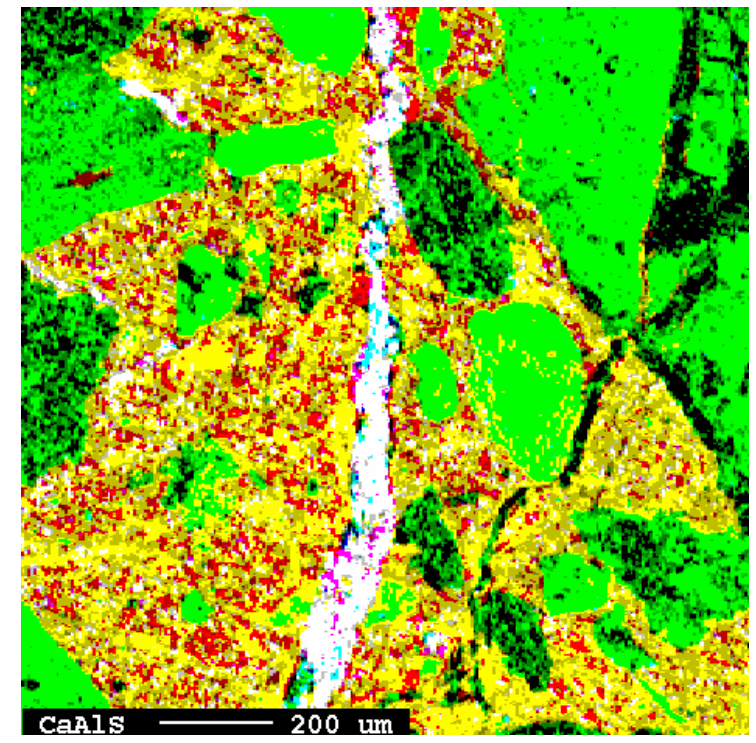

(b) area B in Fig. 11

white: ettringite containing $\mathrm{Ca}, \mathrm{C}, \mathrm{O}$, green: aggregate with $\mathrm{Al}$, black: area without $\mathrm{Ca}, \mathrm{Al}, \mathrm{S}$, yellow: matrix with $\mathrm{Ca}, \mathrm{Al}$

Fig. 12 Distribution of ettringite by RGB method. 
along the matrix. On the other hand, the green areas exhibiting the presence of epoxy resin can be seen mainly in the spaces between aggregates. It appears that self healing was difficult in those spaces away from the matrix.

\subsection{Determination of distribution of ettringite by RGB method}

In the cracks inside the pole, besides $\mathrm{CaCO}_{3}$, ettringite $\left(3 \mathrm{CaO} \cdot \mathrm{Al}_{2} \mathrm{O}_{3} \cdot 3 \mathrm{CaSO}_{4} \cdot 32 \mathrm{H}_{2} \mathrm{O}\right)$ was confirmed in many areas. Therefore, mapping of ettringite was attempted by exhibiting the white areas where 3 constituent elements, such as $\mathrm{Ca}$ (red), $\mathrm{Al}$ (green), and $\mathrm{S}$ (blue), are included in the appropriate proportion. The results are shown in Fig. 12. Figure 12(a) is the result of the analysis of area $A$ in Fig. 11 focusing on ettringite. In the same way, Fig. 12(b) is the result of the analysis of area B in Fig. 11 The green areas indicate aggregate with less $\mathrm{Ca}$ and $\mathrm{S}$ but with $\mathrm{Al}$, the black areas indicate epoxy resin without $\mathrm{Ca}$ and $\mathrm{Al}$ and $\mathrm{S}$, the yellow areas indicate matrix with $\mathrm{Ca}$ and $\mathrm{Al}$, and the white areas indicate ettringite.

Figure 12(a) shows the distribution of ettringite precipitated from both crack planes along the crack. Ettringite was mainly observed at the interface between the matrix and crack, and rarely at the interface between the aggregate and crack. Ettringite existed in almost exactly the same areas where $\mathrm{CaCO}_{3}$ was generated in Fig. 11(b). In the precipitates, both ettringite and $\mathrm{CaCO}_{3}$ coexisted. These observation results agree well with the results of observation by powder $\mathrm{X}$ ray diffraction of the precipitate in the crack shown in Fig. 8.

Figure 12(b) shows that the crack at the center with a width of about 50 to $100 \mu \mathrm{m}$ generated in the matrix was almost completely filled with ettringite. On the other hand, the crack near the aggregate in the upper right area remained without any precipitation. As shown in Fig. 10, the constituent elements of ettringite, such as $\mathrm{Ca}, \mathrm{Al}$, and $\mathrm{S}$, are widely distributed in the matrix. When cracks were generated in the matrix, the cement paste matrix became the source of components for ettringite, and the ettringite and the monosulfate originally present in the matrix functioned as sites for the precipitation of ettringite. On the other hand, the necessary components for ettringite were not supplied from aggregate, and as ettringite and monosulfate nuclei are not present in any large quantity in aggregate, ettringite was not observed in the cracks near aggregate.

\subsection{Condition of steel}

Figure 13 shows the condition of the PC steel. While a small amount of corrosion was observed, no loosened rust was detected. Severe corrosion was prevented during 34 years of service because the crack from the surface of the pole to the steel was healed by $\mathrm{CaCO}_{3}$ and ettringite, restraining the ingress of corrosive substances. The effect of crack healing in terms of restraining steel corrosion should be investigated further in the future.

\section{Discussion of crack healing mechanism}

$\mathrm{CaCO}_{3}$ was observed at the surface of the pole, and $\mathrm{CaCO}_{3}$ and ettringite were observed in the cracks inside the pole. Judging from the site and the condition of precipitation of $\mathrm{CaCO}_{3}$, water must have flowed from inside of the pole to the surface through the cracks. During that time, $\mathrm{Ca}$ ion was supplied from the matrix into the water, reacted with $\mathrm{CO}_{2}$ in the air and precipitated as $\mathrm{CaCO}_{3}$.

$\mathrm{CaCO}_{3}$ was observed also inside the pole. In concrete utility poles, the rate and depth of carbonation are modest, however it has been reported that carbonation can be observed along cracks when they exist (Ueda et al. 2007). In the specimen analyzed in this research, the same phenomenon happened. $\mathrm{CaCO}_{3}$ was observed in large amounts near the interface between the matrix and crack, where the $\mathrm{Ca}$ concentration was lower. It has been reported that there is an appropriate $\mathrm{pH}$, or that the change in $\mathrm{pH}$ is important, for precipitation of $\mathrm{CaCO}_{3}$ (Edvardsen 1999; Stark and Wicht 1995). In the specimen in this research also, the matrix was the source for $\mathrm{Ca}$ ion, and the $\mathrm{pH}$ of the matrix became lower due to the leaching of $\mathrm{Ca}$ and alkaline metals, which led to a good environment for the precipitation of $\mathrm{CaCO}_{3}$.

Ettringite was also observed mainly near the interface between the matrix and crack. Components for ettringite such as $\mathrm{Ca}^{2+}, \mathrm{Al}^{3+}$, and $\mathrm{SO}_{4}{ }^{2-}$ were supplied from the matrix, and developed into 50 to $100 \mu \mathrm{m}$ long needle shaped crystals. Furthermore, minute particles, which seemed to be $\mathrm{CaCO}_{3}$ were observed in the midst of the needle shaped crystals. The ettringite in needle shaped crystal with a large surface area may have functioned as sites for the precipitation of $\mathrm{CaCO}_{3}$, accelerating clogging of the cracks. This kind of combined healing mechanism seems similar to the healing of concrete with short fiber where the precipitation of $\mathrm{CaCO}_{3}$ was accelerated in cracks due to the existence of short fibers serving as precipitation sites (Homma et al. 2009).

Concrete utility poles, which are pre-tensioned spun concrete poles, are cast with centrifugal force. During the

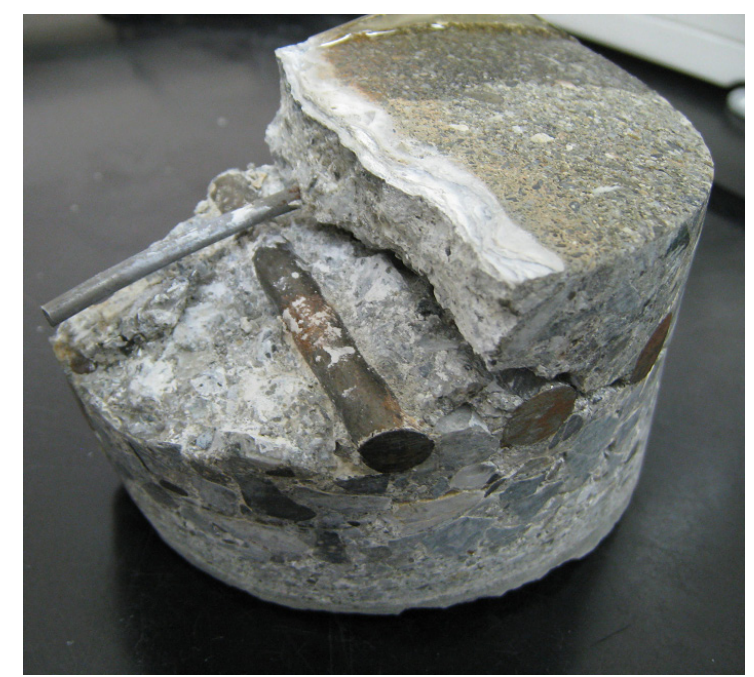

Fig. 13 Condition of PC steel behind the healed crack. 
manufacturing process, cement particles with large diameter and with higher density than other constituents gather near the surface of the pole and remain unhydrated, possibly contributing to the precipitation of calcite and ettringite.

On the other hand, aggregate of larger diameter also tends to gather to the surface of the pole. When cracks are generated in a pole, there is a large possibility that the cracking will occur at the interface between aggregate and the matrix, and cracks along aggregate are difficult to heal. Cracks about $200 \mu \mathrm{m}$ wide are difficult to heal completely, and therefore controlling the crack width is also important for sufficient self healing.

\section{Conclusions}

The following was confirmed from the analysis of a core taken from a concrete utility pole which had been in service for 34 years in a freezing and thawing environment in Shiga Prefecture in Japan. Longitudinal cracks in the pole were naturally healed, and the core taken at the height of $1.7 \mathrm{~m}$ from the ground was analyzed to understand the mechanism of self healing.

(1) Cracks connecting the PC steel to the surface of the pole were generated. The crack about $200 \mu \mathrm{m}$ wide was not completely healed, but the cracks with a width of 50 to $100 \mu \mathrm{m}$ were filled up with precipitates.

(2) At the surface of the pole, calcite, a kind of $\mathrm{CaCO}_{3}$, was observed. Judging from the condition of the precipitation, it was guessed that water accumulated inside the pole had flowed through the crack to the surface.

(3) Calcite and ettringite had precipitated inside the cracks in the pole. These precipitates were mainly observed at the interface between the matrix and crack, and much less at the interface between the aggregate and crack.

(4) Ettringite precipitated in the cracks inside the pole had needle shaped crystals pointing mainly in one direction. Minute particles of $\mathrm{CaCO}_{3}$ were observed near the ettringite. The ettringite functioned as a site for the precipitation of $\mathrm{CaCO}_{3}$.

(5) The PC steel at the crack did not exhibit severe corrosion. This might be due to self healing of the crack.

\section{References}

Edvardsen, C., (1999). "Water permeability and autogenous healing of cracks in concrete." $A C I$ material Journal, 96(4), 448-454.

Homma, D., Mihashi, H. and Nishiwaki, T., (2009). "Self-healing capability of fiber reinforced cementitious composited." Journal of Advanced Concrete Technology, 7(2), 217-228.

JCI, (2009). "Report of the technical committee on autogenous-healing in cementitious materials." (in Japanese)

JCI, (2011). "Report of the technical committee on self-healing / repairing technology in cement-based materials." (in Japanese)

Mohammed, T. U. and Hamada, H., (2008). "Healing of voids, cracks and joints in concrete exposed under marine environments." Concrete Journal (JCI), 46(3), 25-30. (in Japanese)

Stark, J. and Wicht, B., (1995). "Dauerhaftigkeit von Beton." Schriften der Bauhaus Weimar - Universiat, Nr. 100, Japanese translation by Ohta, T. and Saeki, N., Japan Cement Association, 1999, 57-60.

Ueda, H., Kudo, T. and Sasaki, T., (2004). "Diagnosis and maintenance of deteriorated concrete utility poles." RTRI report, 18(10), 3-8. (in Japanese)

Yoshida, H., (2007). "Investigation on deteriorated conditions of prestressed concrete utility poles." Concrete Journal (JCI), 45(8), 75-78, (in Japanese).

Watanabe, Y., Hosoda, A., Higuchi, T., Yoshida, H. and Aoki, H., (2011). "Investigation on generation and development of longitudinal cracks in PC telegraph poles." Proceedings of the Concrete Structure Scenarios, JSMS, 11, 513-520. (in Japanese) 


\title{
Recovery of Protective Performance of Cracked Ultra High Performance-Strain Hardening Cementitious Composites (UHP-SHCC) Due to Autogenous Healing
}

\author{
Minoru Kunieda ${ }^{1}$, Kang Choonghyun ${ }^{2}$, Naoshi Ueda ${ }^{3}$ and Hikaru Nakamura ${ }^{4}$
}

Received 26 December 2011, accepted 17 September 2012

doi:10.3151/jact.10.313

\begin{abstract}
A new strain hardening cementitious composite with a dense matrix, Ultra High Performance-Strain Hardening Cementitious Composites (UHP-SHCC), has been developed. This material combines excellent protective performance with a significantly higher tensile strength and strain hardening at tensile strength. Further, the material has controlled fine cracks (less than 30 microns). A low water to binder ratio with silica fume that causes a pozzolanic reaction is used in UHP-SHCC. These characteristic may give advantages for autogenous healing after the cracking.

This paper presents autogenous healing behavior of cracked UHP-SHCC, and discusses about recovery of protective performance through air and water permeability test results. It was confirmed that UHP-SHCC has potentially autogenous healing properties. The air permeability coefficient and water permeation were dramatically decreased by increasing of re-curing period. Re-curing in water was more effective than re-curing in air for recovery. The effect of induced damage level on recovery of the used indices was not significant, because crack width was controlled and was almost the same among all the cracks. The repeatability of autogenous healing (twice in this study) was confirmed.
\end{abstract}

\section{Introduction}

Cracks are one of the causes of deterioration of concrete structures, and many efforts such as inspection and repair are being made in this area. Autogeneous healing of cementitious material may be helpful to reduce inspection and repair work within a maintenance framework, because the autogenous healing phenomena include crack sensing and crack closing. In recent years, state-of-the art reports have been compiled to accelerate the pace of research on autogeneous healing and its applications (Igarashi et al. 2009; de Rooij and Schlangen 2011), and work has been done in the areas of terminology definition and phenomena classification. The Japan Concrete Institute has defined autogenous healing that causes crack closure due to the original compositions of concrete (Igarashi et al. 2009). For instance, crystallization of calcium carbonate, which is a main mechanism reported by Edvardsen (1999), hydration of un-hydrated cement, and pozzolanic reaction are involved in autogenous healing.

Controlled crack width is also one of the key issues in autogeneous healing phenomena. Various kinds of re-

\footnotetext{
${ }^{1}$ Associate Professor, Dept. of Civil Engineering, Nagoya University, Japan.

E-mail: kunieda@nagoya-u.jp

${ }^{2}$ Graduate Student, Dept. of Civil Engineering, Nagoya University, Japan.

${ }^{3}$ Assistant Professor, Dept. of Civil Engineering, Nagoya University, Japan.

${ }^{4}$ Professor, Dept. of Civil Engineering, Nagoya University, Japan.
}

coveries due to autogenous healing are reported, such as recovery of strength, stiffness of members (Schlangen et al. 2006), and water tightness (Reinhardt and Jooss 2003), and most former research concluded that the capability of recovery due to healing depends on the induced crack width in concrete.

Adding short fiber is one of the effective methods to reduce (control) crack width in concrete. Regarding fiber reinforced cementitious materials including strain hardening type materials with multiple fine cracks, Li et al. (1998) reported experimental results on the self-healing of Engineered Cementitious Composite (ECC), describing the recovery of stiffness through resonance frequency. Yang et al. (2009) also reported on the recovery of stiffness in ECC under wet and dry cycles. Homma et al. (2009) investigated the self-healing phenomena of different types of FRCCs including a hybrid fiber system, and they confirmed the recovery of water tightness and tensile strength.

\section{Autogenous healing of UHP-SHCC and its advantages}

A new strain hardening cementitious composite, Ultra High Performance-Strain Hardening Cementitious Composite (UHP-SHCC), has a dense matrix, a significantly higher tensile strength and strain hardening at tensile strength (Kunieda 2007, 2011; Kamal 2008). Figure 1 shows examples of tensile stress and strain response of UHP-SHCC, for different fiber volumes (Kamal 2008). This material can also produce a multiple fine cracks in tension, as shown in Fig. 2.

UHP-SHCC was expected to have the following advantages for autogenous healing. 


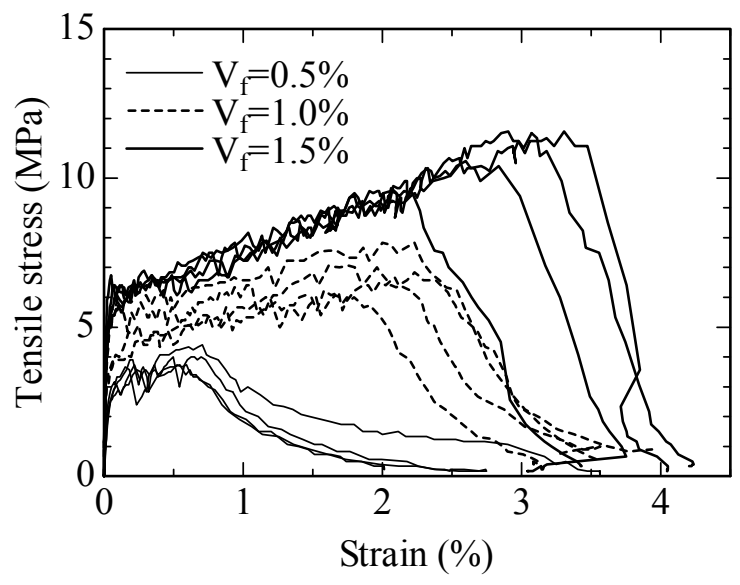

Fig. 1 Examples of tensile stress - strain response with different fiber content (Kamal 2008).

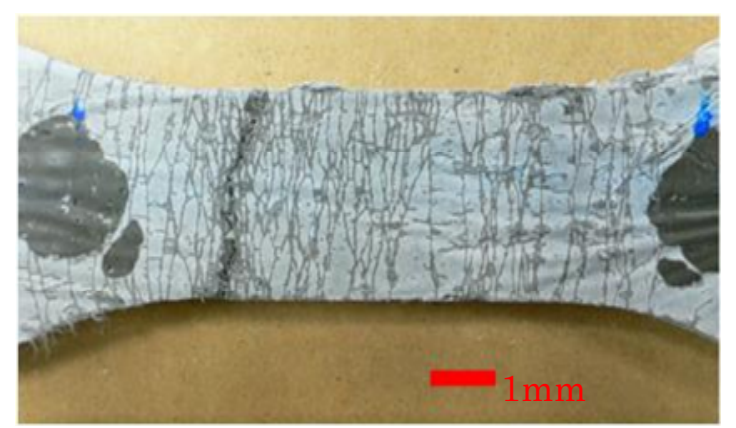

Fig. 2 Controlled fine cracks of UHP-SHCC.

(1) It is well known that autogenous healing works best for fine crack widths, as demonstrated in ECC ( $\mathrm{Li}$ et al. 1998). The width of cracks in UHP-SHCC used in this study was smaller than that of ordinary Strain Hardening Cementitious Composites (SHCC) including ECC, because a high strength matrix and high stiffness polyethylene fiber (nominal elastic modulus: $88 \mathrm{GPa}$ ) were used.

(2) Silica fume was used in UHP-SHCC under this study to allow activation of the pozzolanic reaction, contribution to autogenous healing. This is a similar mechanism to that in ordinary SHCC that includes fly ash.

(3) The low water to binder ratio (less than 0.22) of UHP-SHCC can be expected to yield much un-hydrated cement during the service period.

Based on the above material characteristics, autoge- nous healing of UHP-SHCC can readily expected.

On the other hand, the following aspects were identified as requiring clarification.

(1) UHP-SHCC has been used as a repair material in surface coating repair by using a low water to binder ratio. The protective performance of UHP-SHCC is much higher than that of ordinary SHCC. This indicates that the protective performance is dramatically decreased after cracking. Although recovery of permeability has been observed in former research on ordinary SHCC, recovery of higher protective performance caused by low water to binder ratio should be confirmed. Note that UHP-SHCC has higher strength and higher strain capacity in tension, and the recovery of stiffness and strength is not discussed in this study.

(2) In ordinary SHCC, PVA fiber which has a hydroxide on the surface of the fiber, is normally used. Nishiwaki (2012) reported that PVA fibers become nuclei of healing product. Polyethylene fiber, however, does not have hydroxide, and thus offers lesser autogenous healing capability compared with ordinary SHCC with PVA fiber.

(3) Existing research reports the recovery of damage that was induced once. The repeatability of autogenous healing of UHP-SHCC should be confirmed.

This paper presents the results of an investigation on recovery of protective performance of UHP-SHCC due to autogenous healing phenomena through air and water permeability tests, in order to clarify the above points. In particular, UHP-SHCC with different damage levels were tested, and recovered specimens were loaded and cured again to confirm the repeatability of autogenous healing.

\section{Experimental program}

\subsection{Specimens}

Table 1 lists the mix proportions of UHP-SHCC used in this study. The water to binder ratio (W/B) was 0.22 . Low heat Portland cement (density: $3.14 \mathrm{~g} / \mathrm{cm}^{3}$ ) was used, and $15 \%$ of the cement was replaced by silica fume (density: $2.2 \mathrm{~g} / \mathrm{cm}^{3}$ ). Quartz sand (less than $0.5 \mathrm{~mm}$ in diameter, density: $2.68 \mathrm{~g} / \mathrm{cm}^{3}$ ) was used as the fine aggregate. Fiber volume of $1.5 \%$ was used in this study. High strength polyethylene (PE) fiber (density: $0.97 \mathrm{~g} / \mathrm{cm}^{3}$, tensile strength: $2700 \mathrm{MPa}$, elastic modulus: $88 \mathrm{GPa}$ ) was used for UHP-SHCC. The diameter and length of the PE fibers were $0.012 \mathrm{~mm}$ and $6 \mathrm{~mm}$, re-

Table 1 Mix proportions of used UHP-SHCC.

\begin{tabular}{|c|c|c|c|c|c|c|c|c|}
\hline \multirow{2}{*}{$\begin{array}{c}\text { Fiber } \\
\text { volume } \\
\mathrm{V}_{\mathrm{f}}(\%)\end{array}$} & \multirow{2}{*}{ W/B } & \multicolumn{7}{|c|}{ Unit content $\left(\mathrm{kg} / \mathrm{m}^{3}\right)$} \\
\cline { 3 - 9 } & & Water & Cement & Silica fume & Sand & SP & ARA & Fiber \\
\hline 1.5 & 0.22 & 340 & 1313 & 232 & 155 & 15.4 & 0.062 & 14.6 \\
\hline
\end{tabular}

SP: Superplasticizer

ARA: Air reducing agent 
spectively. Superplasticizer (poly-carbon acid type) was used to enhance the workability of the material, and air reducing agent was used.

Uniaxial tensile tests were conducted by using dumbbell-shaped specimens (tested cross section: $10 \times 30 \mathrm{~mm}$ ) for UHP-SHCC at the age of 28days. Table 2 shows the averaged tensile strength and strain at the tensile strength.

Figure 3 shows the shape of the specimen used for the investigation on the autogenous healing properties of UHP-SHCC. Plate-shaped specimens having the cross section of $150 \mathrm{~mm} \times 30 \mathrm{~mm}$ were prepared. There were two re-bars of D6 $\left(\mathrm{SD} 295, \mathrm{f}_{\mathrm{y}}=407 \mathrm{MPa}\right)$ in the specimen to impart stable crack formation. Re-bars of D10 (SD295A) were also embedded in both specimen ends to allow holding of the specimen by the testing machine.

\subsection{Air permeability test}

Air permeability tests were conducted using the Torrent Permeability Tester (TPT), proposed by Torrent (1992). This is an absolutely non-destructive method to check the denseness of the cover concrete and to evaluate the durability of concrete structures. The Torrent Permeability Tester device is shown in Fig. 4. The device consists of a chamber, a vacuum pump, a pressure sensor, and a logger. In the measurement, the chamber is put on the concrete surface. The chamber has two cells, an inner chamber and an outer chamber. Equalizing the pressure of both chambers, it is possible to make a unidirectional air flow. The air permeability coefficient is calculated according to the model of Torrent (1992). In this study, air permeability tests were performed on circular areas at the center of the specimens.

All specimens cured in water were taken out from water, and kept in air condition ( 20 degree C) for 2 days. After the pretreatment, the air permeability test was
Table 2 Tensile properties of used UHP-SHCC (28days).

\begin{tabular}{|c|c|}
\hline Tensile strength $(\mathrm{MPa})$ & Strain at tensile strength (\%) \\
\hline 6.8 & 1.04 \\
\hline
\end{tabular}
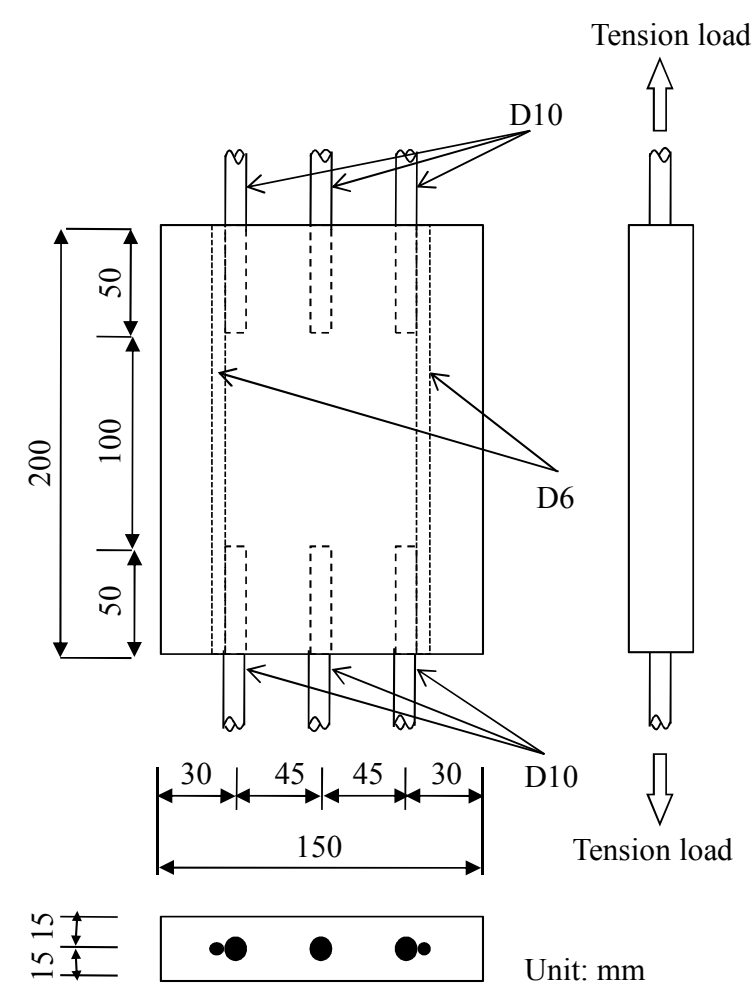

Fig. 3 Shape of specimen for accessing autogenous healing.
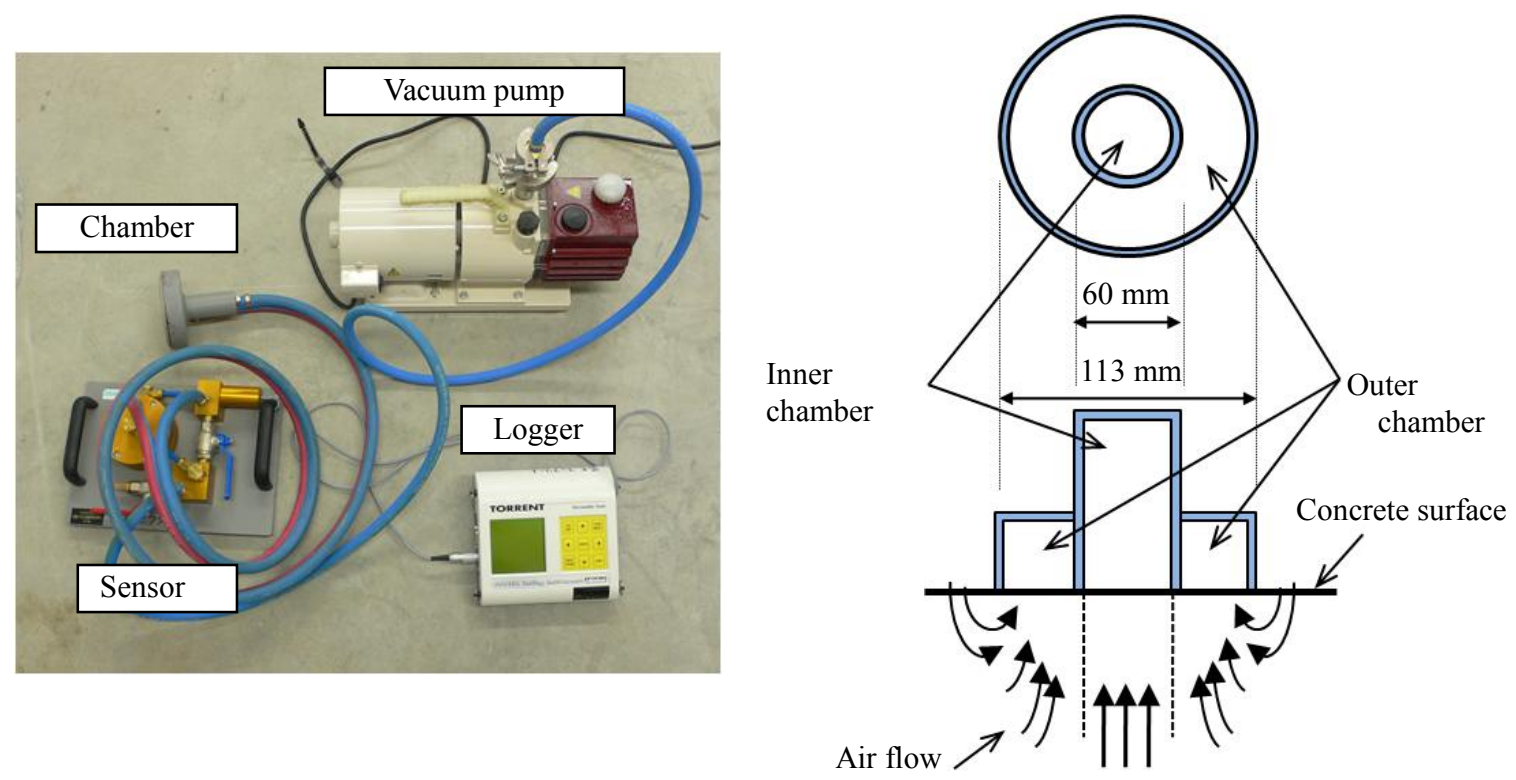

Fig. 4 Torrent permeability tester. 
conducted. The specific water content of each specimen was not measured at the time of testing.

\subsection{Water permeability test}

In this study, the surface penetrant test method recommended by the Japan Society of Civil Engineers (JSCE 2004) was used. Figure 5 shows the outline of the testing method. A caliber funnel $75 \mathrm{~mm}$ in diameter connected to a measuring pipette by a rubber tube was attached to the surface of the specimen and water was poured until the water head of $250 \mathrm{~mm}$. Then, the change in water head was measured after about 20 hours. The amount of water per hour was calculated, and it was defined as water permeation in this study. The interface between the funnel and the surface of specimen was sealed with a silicone sealant. The tests were performed on circular areas at the center of the specimens, which was the same position used in the air permeability tests. Since the specimens after the air permeability tests were used for the water permeability tests, the specimens were exposed in dry condition (20 degree C) after having been taken out from water (water curing).

\subsection{Induced cracks and re-curing method}

The experimental procedures are summarized in Fig. 6. The specimens were demoulded at one day after casting and cured in water at constant temperature room (20 degree C). Then air permeability tests and water permeability tests were conducted at the age of 32 to 35 days. Then at the age of 36 to 37 days, cracks were induced by uniaxial tensile test, as shown in Fig. 7. Loading was monitored by LVDT fixed on both specimen surfaces (measurement length: 150mm). A schematic image of stress-strain curves of the loaded specimens is shown in Fig. 8. Each specimen was loaded until the tensile strain of either $0.1 \%$ or $0.2 \%$, and then unloaded. Typical crack patterns are shown in Fig. 9. The averaged crack width $\bar{w}$ was calculated by Eq. 1 .

$$
\bar{w}=\frac{\varepsilon_{r e s} \times \Delta \ell}{n}
$$

where,

$\bar{w}:$ Averaged crack width

$\varepsilon_{\text {res }}:$ Residual strain

$\Delta \ell:$ Measurement length $(150 \mathrm{~mm})$

$n$ : Number of cracks in measurement length

After the tensile tests, air permeability tests and the water permeability tests were conducted again, and the specimens were cured in air or in water at a constant temperature (20 degree C) for 20 days, 90days and 360days.

Table 3 lists the specimen series, unloaded strain, re-curing conditions and re-curing periods. The number of specimens in each condition was 3 to 4 . Three control specimens with no damage were also prepared. Table 4 tabulates the obtained residual strain, number of cracks, and averaged crack width of each cracked specimen. Several cracks were observed in the measurement area.

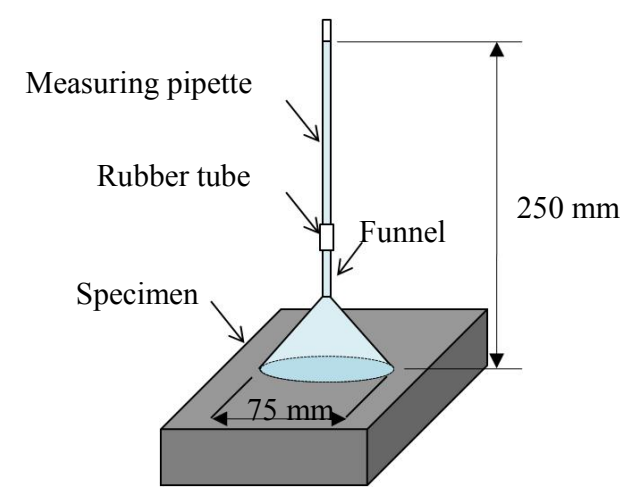

Fig. 5 Water permeability test setup.

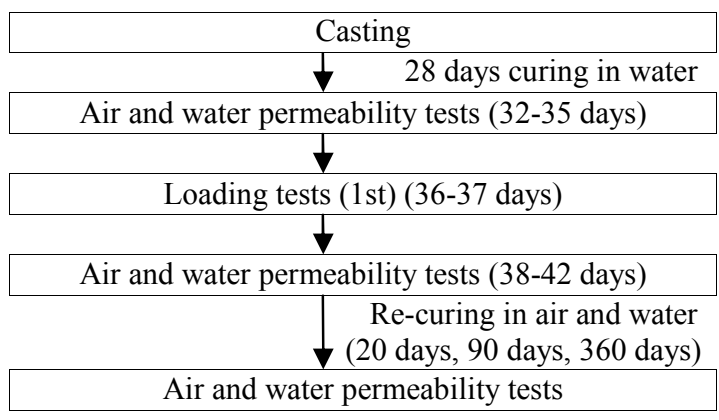

Fig. 6 Experimental procedures.

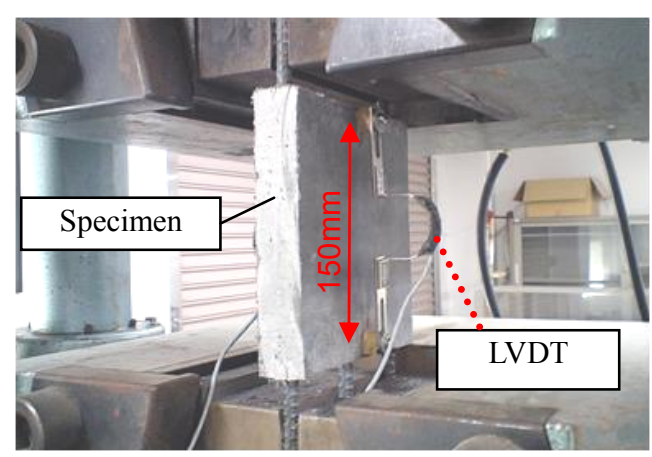

Fig. 7 Uniaxial tensile test.

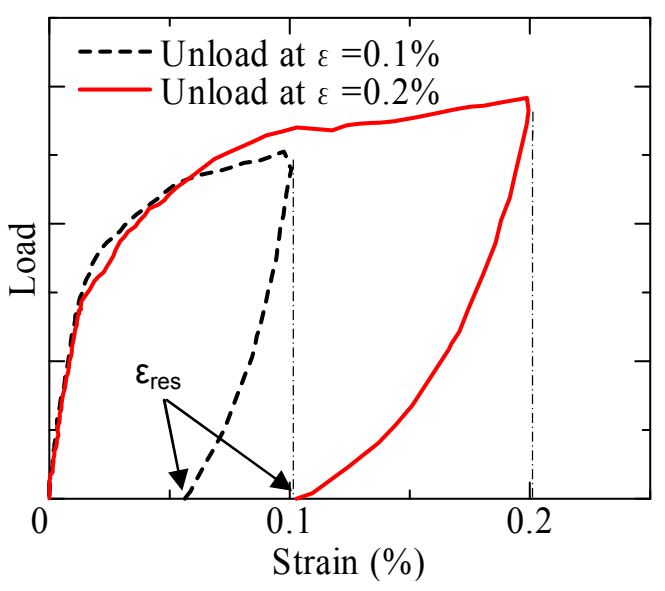

Fig. 8 Schematic image of loading manner. 


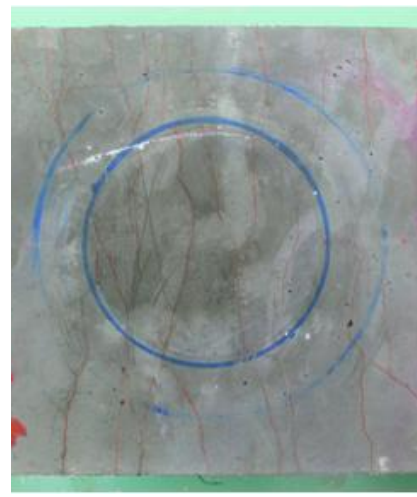

(a) Unloaded at $0.1 \%$ strain

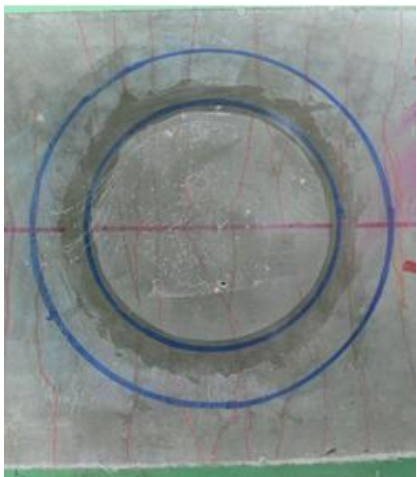

(b) Unloaded at $0.2 \%$ strain

Fig. 9 Examples of induced cracks.

Table 3 Tested specimens.

\begin{tabular}{|c|c|c|c|}
\hline Series & $\begin{array}{l}\text { Unloaded } \\
\text { strain }(\%)\end{array}$ & $\begin{array}{l}\text { Re-curing } \\
\text { condition }\end{array}$ & $\begin{array}{c}\text { Re-curing } \\
\text { period }\end{array}$ \\
\hline $0 \mathrm{~W}$ & \multirow{2}{*}{$\begin{array}{l}\text { (Without } \\
\text { loading) }\end{array}$} & Water & \multirow{6}{*}{$\begin{array}{l}20 \text { days, } \\
90 \text { days, } \\
360 \text { days }\end{array}$} \\
\hline $0 \mathrm{~A}$ & & Air & \\
\hline $0.1 \mathrm{~W}$ & \multirow{2}{*}{0.1} & Water & \\
\hline $0.1 \mathrm{~A}$ & & Air & \\
\hline $0.2 \mathrm{~W}$ & \multirow{2}{*}{0.2} & Water & \\
\hline $0.2 \mathrm{~A}$ & & Air & \\
\hline
\end{tabular}

Table 4 Induced damage for each specimen.

\begin{tabular}{|c|c|c|c|}
\hline Series & $\begin{array}{c}\text { Residual } \\
\text { strain after } \\
\text { loading (\%) }\end{array}$ & $\begin{array}{c}\text { Number } \\
\text { of cracks }\end{array}$ & $\begin{array}{c}\text { Averaged } \\
\text { crack } \\
\text { width } \\
\text { (micron) }\end{array}$ \\
\hline $0.1 \mathrm{~W}-1$ & 0.051 & 6 & 12.7 \\
$0.1 \mathrm{~W}-2$ & 0.051 & 5 & 15.2 \\
$0.1 \mathrm{~W}-3$ & 0.060 & 4 & 22.3 \\
$0.1 \mathrm{~W}-4$ & 0.052 & 5 & 15.5 \\
\hline $0.1 \mathrm{~A}-1$ & 0.057 & 6 & 14.2 \\
$0.1 \mathrm{~A}-2$ & 0.052 & 6 & 12.9 \\
$0.1 \mathrm{~A}-3$ & 0.051 & 7 & 10.9 \\
\hline $0.2 \mathrm{~W}-1$ & 0.091 & 9 & 15.1 \\
$0.2 \mathrm{~W}-2$ & 0.096 & 14 & 10.3 \\
$0.2 \mathrm{~W}-3$ & 0.114 & 16 & 10.7 \\
\hline $0.2 \mathrm{~A}-1$ & 0.116 & 15 & 11.6 \\
$0.2 \mathrm{~A}-2$ & 0.108 & 15 & 10.8 \\
$0.2 \mathrm{~A}-3$ & 0.081 & 14 & 8.7 \\
\hline
\end{tabular}

The averaged crack width was 8.7 to 22.3 microns. Although the unloaded strain differed, there was no significant difference in averaged crack width, which is the material nature of UHP-SHCC.

\section{Experimental results}

\subsection{Results of air permeability tests}

Figure 10 shows air permeability coefficients of each specimen before loading, just after the loading and after the re-curing for 20 days, 90days and 360days. In the figure, the averaged crack width calculated in Equation 1 is expressed as a dot. Note that the averaged crack width was measured and calculated based on the residual strain at the time of loading test. Crack widths of less than 30 microns were measured, and UHP-SHCC was found to produce fine crack widths, which is one of its advantages for autogenous healing.

In control specimens, air permeability coefficients became smaller with increasing of re-curing period. This decrease seems due to densification of the matrix of UHP-SHCC with increasing age.

The air permeability coefficients of cracked specimens just after loading were over $0.1 \times 10^{-16} \mathrm{~m}^{2}$, and became dramatically larger than those of un-cracked specimens. After re-curing, however, the air permeability coefficients of all cracked specimens, especially those cured in water, became smaller. Some of the air-cured specimens showed decreasing air permeability coefficient, similar to the water-cured specimens. Regarding the difference in re-curing condition, water curing was more effective for autogenous healing, compared with air curing. The main cause for recovery of resistance against air permeability seems to be the formation of products due to re-hydration of cement, pozzolanic reaction and carbonation within induced cracks, with increases in the re-curing period.

Regarding differences in damage level, no significant trend could be identified, because the crack width, which is related to the autogenous healing capability, was almost the same across all cases, as shown in Table 4.

Torrent (1992) also proposed quality grading of concrete and a concrete quality index. In the proposal, an air permeability coefficient of less than $0.1 \times 10^{-16} \mathrm{~m}^{2}$ means good quality concrete, and a coefficient of less than $0.01 \times 10^{-16} \mathrm{~m}^{2}$ indicates very good quality concrete. Note that resistivity of concrete should be used for the total evaluation in his proposal. Through this evaluation, UHP-SHCC recovered by autogeneous healing phenomena has sufficient quality in terms of protective performance. 


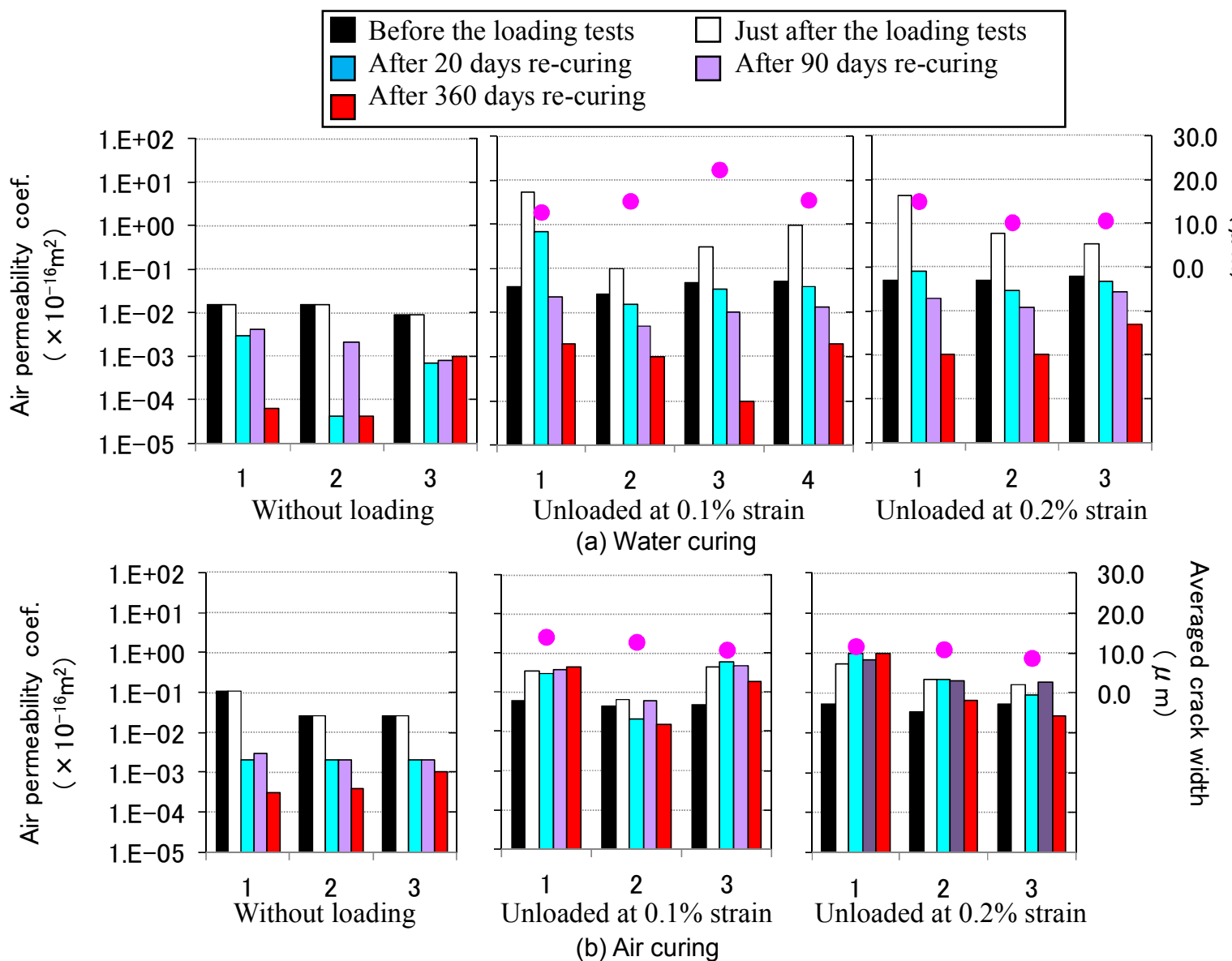

Fig. 10 Results of air permeability tests.

\subsection{Results of water permeability tests}

Figure 11 shows the water permeation of each specimen before loading, just after loading, and after re-curing for 20 days, 90days and 360days. Note that the averaged crack width is represented as a dot in this figure, and that fine cracks having a width of less than 30 microns were observed in UHP-SHCC.

In the control specimens, water permeation decreased with increases in the curing period. It seems that densification of the matrix in UHP-SHCC accelerates the decreases in water permeation with increases in the curing period.

Water permeation of cracked specimens increased dramatically compared with that before loading. After re-curing, however, water permeation decreased in most cracks.

Regarding the difference in re-curing condition, water curing was more effective than air curing for autogenous healing, and there was no significant effect of unloaded strain level on recovery of water permeation. In this study, a similar trend to that of air permeability coefficient was observed experimentally, and it can be concluded that these indices have a similar sensitivity to the recovery of protective performance on autogenous healing of UHP-SHCC. These indices were also affected by moisture content in the test. Further research is needed in this area.

\subsection{Observation of cracked part and material itself}

Figure 12 shows images of cracks observed with a microscope. Figure 12a) is the image of the cracks before re-curing (just after loading), and Fig. 12b) is the image of the cracks after re-curing. The induced cracks were filled with new product, which seems to be hydration products related to contained un-hydrated cement, products due to the pozzolanic reaction of silica fume, and carbonation producing $\mathrm{CaCO}_{3}$.

Figure 13 shows the SEM image of a cracked part. The generated products filling the crack can be observed. The products have a slightly coarse appearance compared with the bulk matrix. Figure 14 shows the results of EDX analysis, with $\mathrm{Ca}$ and $\mathrm{Si}$ detectable not only in the bulk matrix but also in the crack, indicating that the generated products contain $\mathrm{Ca}$ and $\mathrm{Si}$. The products appear caused by crystallization of $\mathrm{Ca}(\mathrm{OH})_{2}$ and pozzolanic reaction. Figure 15 shows the back scattered image of UHP-SHCC at the age of 407 days. The light gray parts represent un-hydrated cement. In the image, half of the cement (about 49\%) remains un-hydrated in the material after 407 days, and it seems that this un-hydrated cement helps the autogenous healing. 


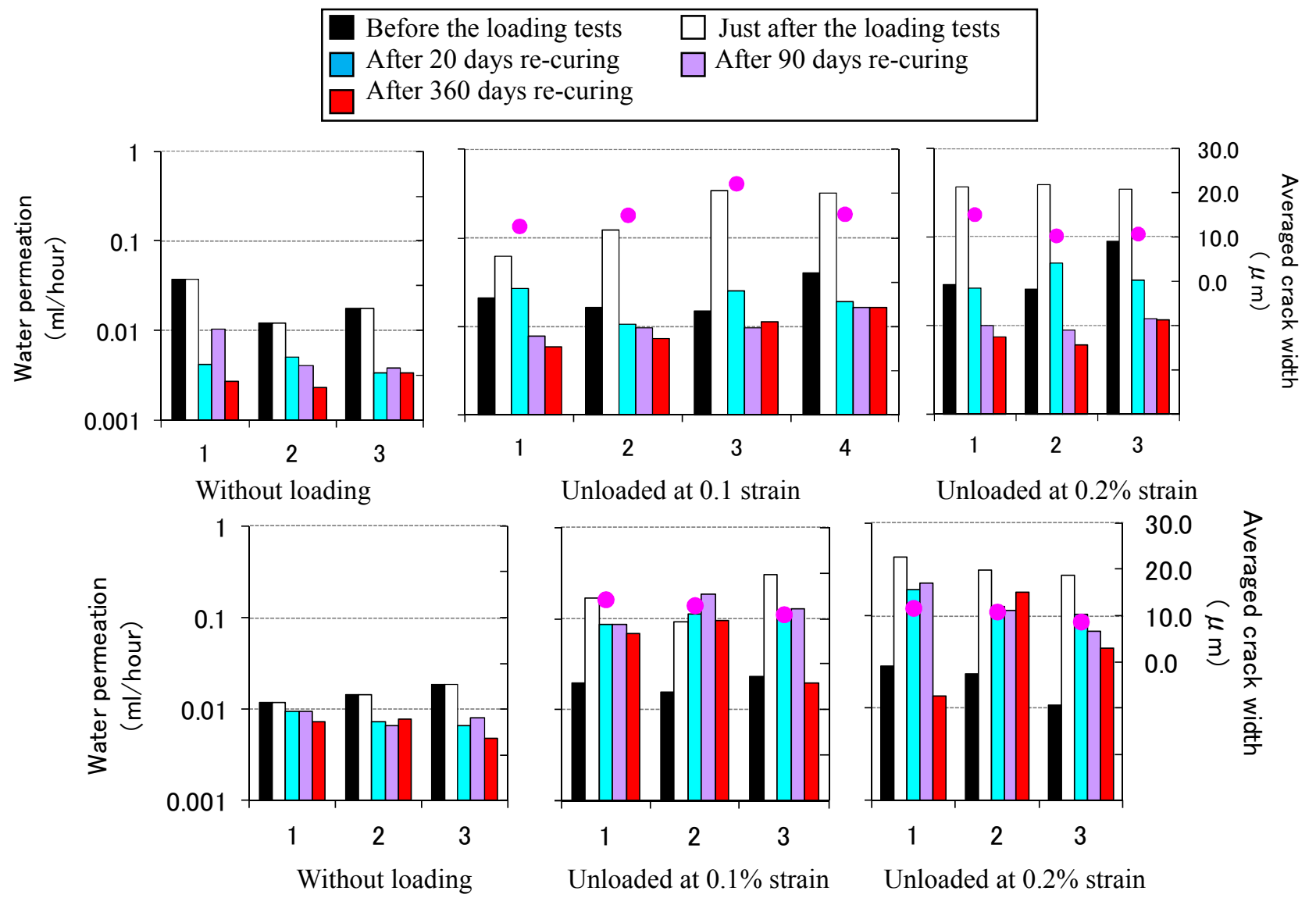

Fig. 11 Results of water permeability tests.

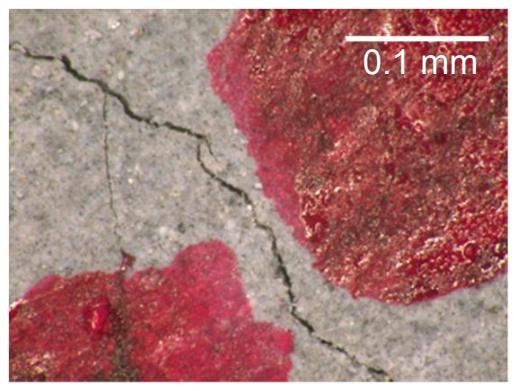

(a) Before re-curing

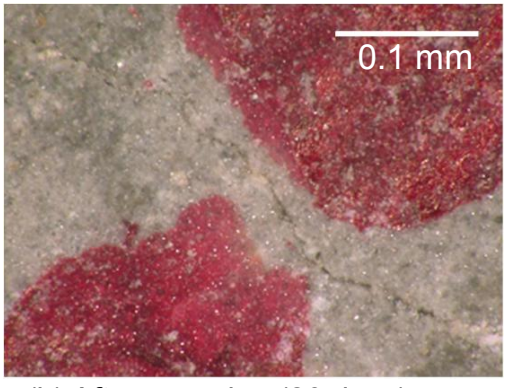

(b) After re-curing (20 days)

Fig. 12 Details of cracks before and after re-curing observed by using microscope (cured in water).

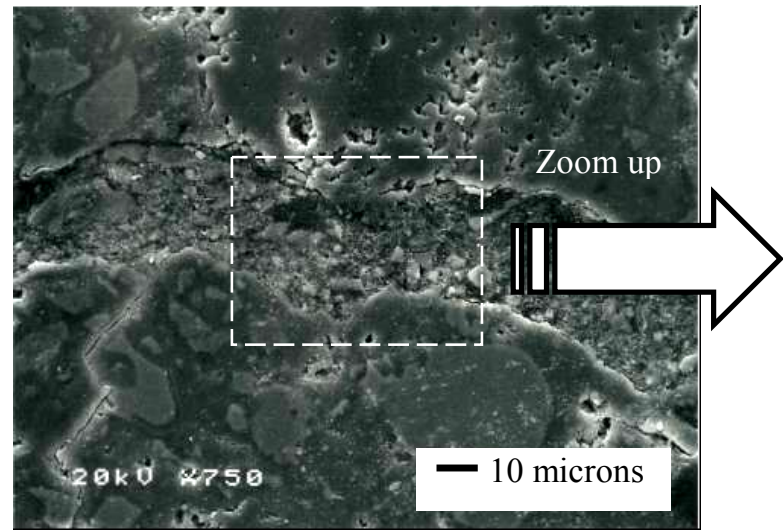

(a) $750 \mathrm{X}$

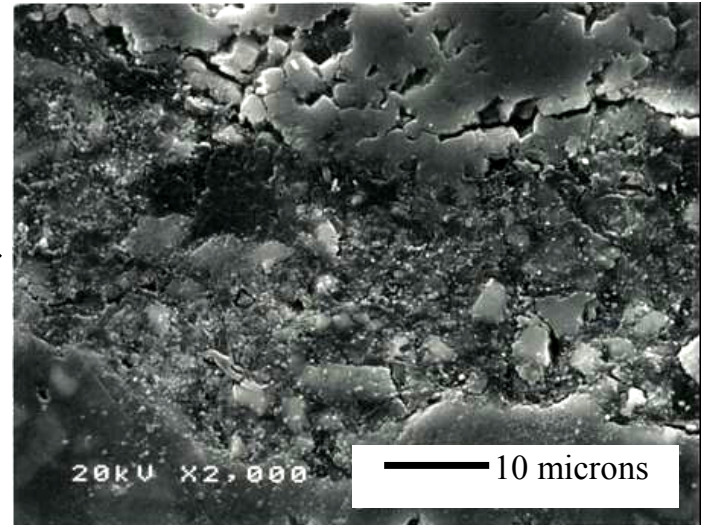

(b) $2000 X$

Fig. 13 Image of cracked part by means of SEM (cured in water). 


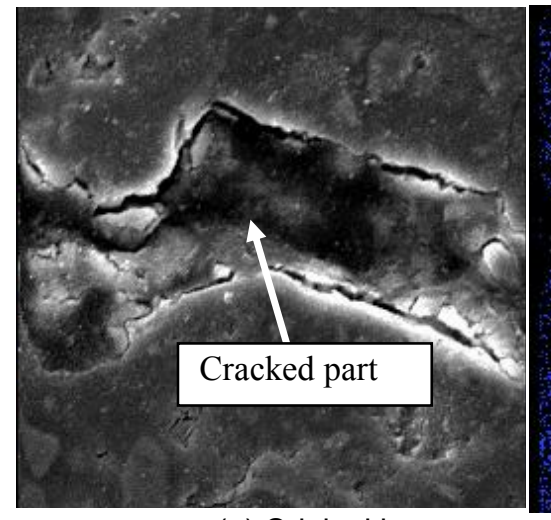

(a) Original image

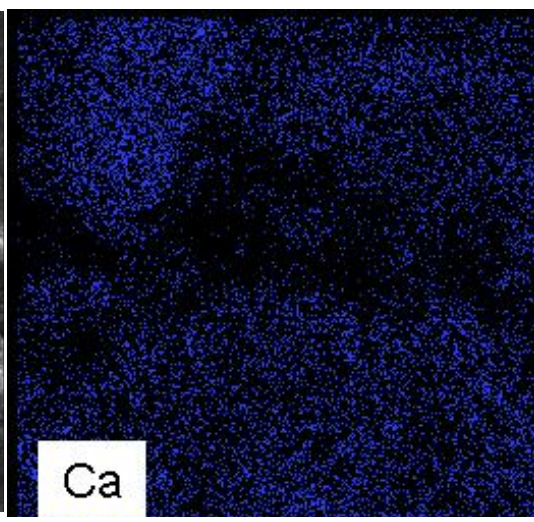

(b) $\mathrm{Ca}$

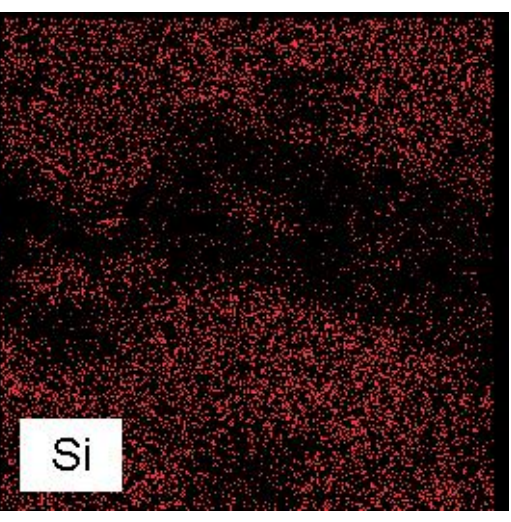

(c) $\mathrm{Si}$

Fig. 14 Image of cracked part by means of energy dispersive X-ray spectrometry (cured in water).

\section{Repeatability of autogenous healing}

\subsection{Outline of experiments}

In this section, the repeatability of autogenous healing was confirmed. The mix proportions of the used UHP-SHCC with fiber volume of $1.5 \%$ are shown in Table 1. An outline of the experiments is given in Fig. 16 Loading tests were carried out twice. In the first loading, load was applied to the strain of $0.1 \%$ or $0.2 \%$, and residual strain was given in each series. In the second loading, load was also provided up to the strain of $0.1 \%$ or $0.2 \%$ again. Here, the unloading point (strain) was the same as that in the first loading. In order to evaluate the recovery of protective performance, air permeability tests and water permeability tests were performed in accordance with the testing method described in the previous section. After each loading test and measurement of air and water permeability, only water curing was conducted. The test series are summarized in Table 5.

Table 6 tabulates the detailed data on residual strain and number of cracks after the first and second loading, respectively. As a result, the number of cracks in the $0.1 \%$ strain series was twice comparing that in the $0.2 \%$ strain series. Averaged crack width was, however, almost the same. After the second loading, a slight increase in the number of cracks was observed in some cases, and averaged crack width became a little larger than after the first loading.

\subsection{Results of air permeability and water per- meability tests}

Figures 17 and 18 show the air permeability coefficients and water permeation of each specimen, respectively. Note that the plus and dot marks in the figure represent the averaged crack width after first loading and second loading, respectively. Significant change in crack width (less than 10 microns) was not observed.

Both air permeability coefficients and water permeation changed dramatically at each loading to induce cracks. The air permeability coefficient could not be measured in the $0.2 \%$ strain series. However, re-curing resulted the reduction of air permeability and water

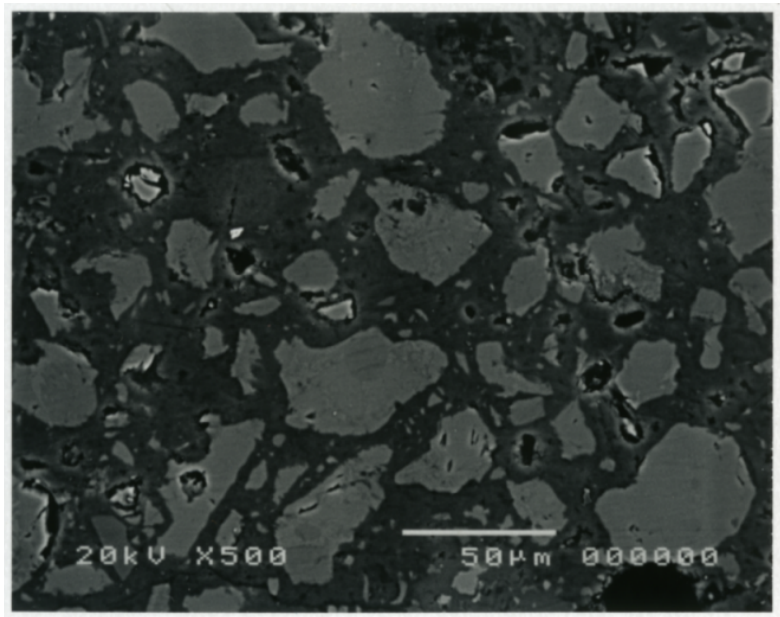

Fig. 15 Back scattered electron image of UHP-SHCC (407 days after casting, hydration ratio: $51.4 \%$ ).

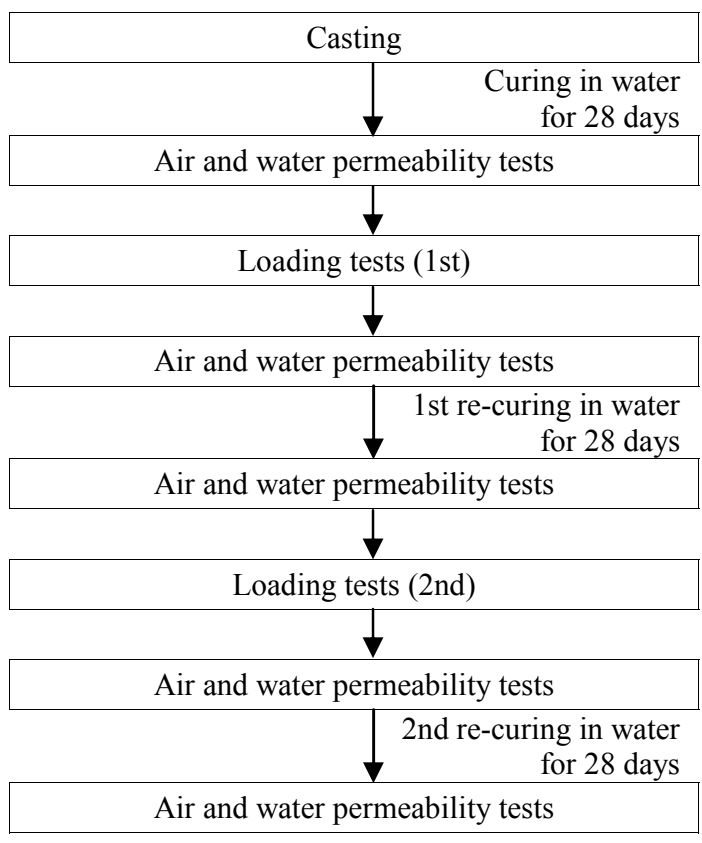

Fig. 16 Outline of the experiments concerning repeatability of autogenous healing. 
Table 5 Tested specimens.

\begin{tabular}{|c|c|c|c|}
\hline Series & Unloaded strain (\%) & $\begin{array}{c}\text { Re-curing } \\
\text { condition }\end{array}$ & Re-curing period \\
\hline $0.1 \mathrm{~W}$ & 0.1 & Water & $\begin{array}{c}\text { 1st re-curing: } 28 \text { days } \\
\text { 2nd re-curing: } 28 \text { days }\end{array}$ \\
\hline $0.2 \mathrm{~W}$ & 0.2 & \\
\hline
\end{tabular}

Table 6 Residual strain and averaged crack width.

\begin{tabular}{|c|c|c|c|c|c|c|}
\hline \multirow{2}{*}{ Series } & \multicolumn{3}{|c|}{ After 1st loading } & \multicolumn{3}{c|}{ After 2nd loading } \\
\cline { 2 - 7 } & $\begin{array}{c}\text { Residual } \\
\text { strain (\%) }\end{array}$ & $\begin{array}{c}\text { Number } \\
\text { of cracks }\end{array}$ & $\begin{array}{c}\text { Averaged crack } \\
\text { width (micron) }\end{array}$ & $\begin{array}{c}\text { Residual } \\
\text { strain (\%) }\end{array}$ & $\begin{array}{c}\text { Number } \\
\text { of cracks }\end{array}$ & $\begin{array}{c}\text { Averaged crack } \\
\text { width (micron) }\end{array}$ \\
\hline $0.1 \mathrm{~W}-1$ & 0.058 & 3 & 28.9 & 0.065 & 4 & 24.4 \\
\hline $0.1 \mathrm{~W}-2$ & 0.049 & 3 & 24.7 & 0.061 & 4 & 22.9 \\
\hline $0.1 \mathrm{~W}-3$ & 0.027 & 2 & 20.4 & 0.069 & 4 & 25.9 \\
\hline $0.2 \mathrm{~W}-1$ & 0.088 & 8 & 16.5 & 0.153 & 8 & 28.7 \\
\hline $0.2 \mathrm{~W}-2$ & 0.100 & 8 & 18.8 & 0.154 & 8 & 28.9 \\
\hline $0.2 \mathrm{~W}-3$ & 0.124 & 7 & 26.6 & 0.144 & 7 & 30.9 \\
\hline
\end{tabular}

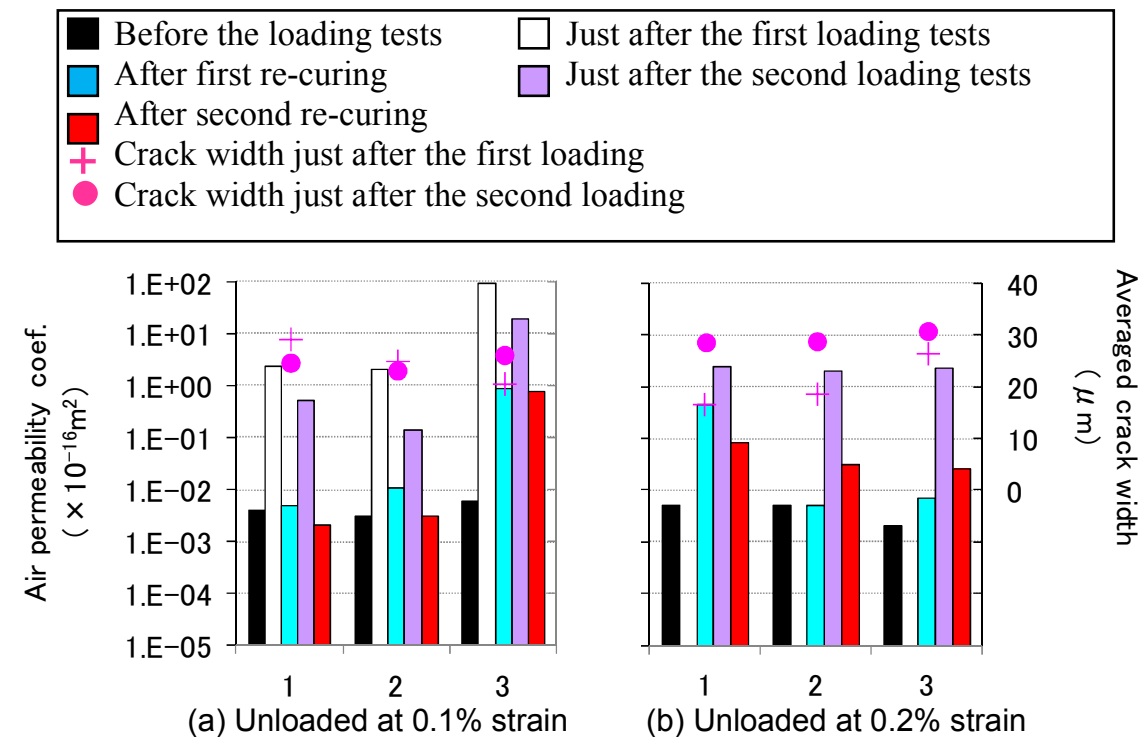

Fig. 17 Results of air permeability tests ( ${ }^{*}$ In the series of $0.2 \%$ strain, air permeability coef. at first loading tests cannot be measured.).

permeation after first re-curing and second one. Consequently, the used re-curing period (28 days) seems to be sufficient to recover up to the same level as the control specimen (without loading) under water curing conditions. In this study, the reduction of air and water permeability was observed twice, and the repeatability of the autogenous healing was confirmed.

\section{Conclusions}

The autogenous healing phenomena for protective performance were experimentally investigated, and the following conclusions were obtained.

(1) It was confirmed that the UHP-SHCC has potentially autogenous healing properties. The air permeability coefficient and water permeation were dramatically decreased with increases in the re-curing period. Re-curing in water was particularly effective for recovery, compared with re-curing in air. Eventually, recovery of protective performance was possible under water curing conditions, even if a higher protective performance level is associated with a low water to binder ratio.

(2) The effect of induced damage level on recovery of the used indices (air permeability coefficient and water permeation) was not significant because crack width was almost constant throughout the material, although the number of crack differed.

(3) In this study, recovery of protective performance, which was represented by reduction of air and water permeability, was observed twice, and the repeatability of the autogenous healing was confirmed.. 


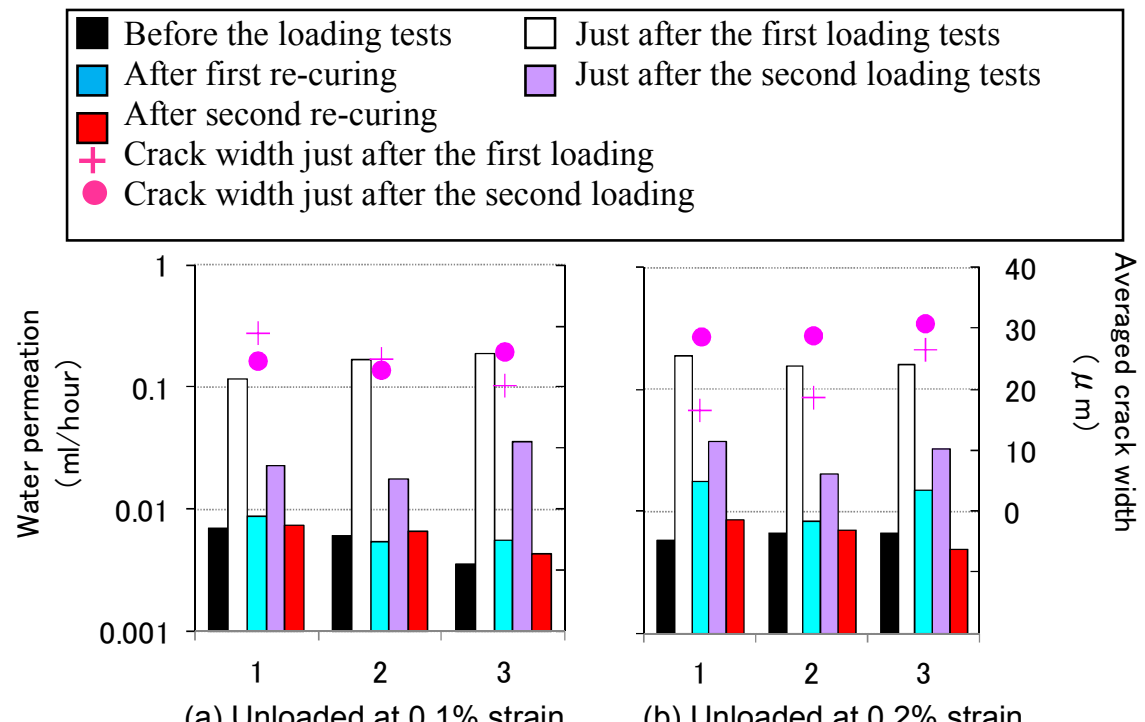

Fig. 18 Results of water permeability tests.

\section{Acknowledgement}

This research was supported by Grant-in-Aid for Scientific Research (Grant-in-Aid for Young Scientists (B) 19760302). The authors are grateful for this support.

\section{References}

De Rooij, M. R. and Schlangen, E., (2011). "Self-healing phenomena in cement-based materials." Draft of State-of-the-Art report of RILEM Technical Committee 221-SHC.

Edvardsen, C., (1999). "Water permeability and autogenous healing of cracks in concrete." $A C I$ Material Journal, 448-454.

Homma, D., Mihashi, H. and Nishiwaki, T., (2009). "Self-healing capability of fibre reinforced cementitious composites." Journal of Advanced Concrete Technology, 7(2), 217-228.

Igarashi, S., Kunieda, M. and Nishiwaki, T., (2009). "Research activity of JCI Technical Committee TC-075B: Autogenous healing in cementitious materials." In: Proceedings of 4th International Conference on Construction Materials: Performance, Innovations and Structural Implications, Nagoya August 2009, 89-96.

Japan Society of Civil Engineers, (2005). "Test methods of surface penetrant (draft)." Recommendations for design and construction about construction method of surface protection (Concrete Library of JSCE 119), 58-59.(in Japanese)

Kamal, A., Kunieda, M., Ueda, N. and Nakamura, H., (2008). "Evaluation of crack opening performance of a repair material with strain hardening behavior." Cement and Concrete Composites, 30 (10), 863-871.

Kunieda, M., Denarie, E., Bruehwiler, E. and Nakamura, H., (2007). "Challenges for strain hardening cementitious composites- deformability versus matrix density." In: H.W. Reinhardt and A. Naaman Eds. 5th International RILEM Workshop on High Performance Fiber Reinforced Cement Composites, Mainz 2007, 31-38.

Kunieda, M., Ogura, H., Ueda, N. and Nakamura, H., (2011). "Tensile fracture process of Strain Hardening Cementitious Composites by means of three-dimensional meso-scale analysis." Cement and Concrete Composites, 33(9), 956-965.

Li, V. C., Lim, Y. M. and Chan, Y. W., (1998). "Feasibility study of a passive smart self-healing cementitious composite." Composites Part B, 29B, 819-827.

Nishiwaki, T., Koda, M., Yamada, M., Mihashi, H. and Kikuta, T., (2012). "Experimental study on self-healing capability of FRCC using different types of synthetic fibers." Journal of Advanced Concrete Technology, 10, 195-206.

Reinhardt, H. W. and Jooss, M., (2003). "Permeability and autogenous healing of cracked concrete as a function of temperature and crack width." Cement and Concrete Research, 33(7), 981-985.

Schlangen, E., Heide, N. and van Breugel K., (2006). "Crack healing of early age cracks in concrete." In: M.S. Konsta-Gdoutos Ed. Measuring, Monitoring and Modeling Concrete Properties, Springer, 273-284.

Torrent, R., (1992). "A two-chamber vacuum cell for measuring the coefficient of permeability to air of the concrete cover on site." Material and Structures, 25, 358-365.

Yang, Y., Lepech, M. D., Yang, E. H. and Li, V. C., (2009). "Autogenous healing of engineered cementitious composites under wet-dry cycles." Cement and Concrete Research, 39, 382-390. 